\title{
Radioactive decays at limits of nuclear stability
}

\author{
M. Pfützner* and M. Karny \\ Faculty of Physics, \\ University of Warsaw, \\ Hoża 69, PL-00-681 Warszawa, \\ Poland \\ L.V. Grigorenko \\ Flerov Laboratory of Nuclear Reactions, \\ JINR, RU-141980, Dubna, \\ Russia \\ K. Riisager \\ Department of Physics and Astronomy, \\ Aarhus University, \\ DK-8000 Aarhus C, \\ Denmark
}

(Dated: November 3, 2011)

The last decades brought an impressive progress in synthesizing and studying properties of nuclides located very far from the beta stability line. Among the most fundamental properties of such exotic nuclides, usually established first, is the half-life, possible radioactive decay modes, and their relative probabilities. When approaching limits of nuclear stability, new decay modes set in. First, beta decays become accompanied by emission of nucleons from highly excited states of daughter nuclei. Second, when the nucleon separation energy becomes negative, nucleons start to be emitted from the ground state. Here, we present a review of the decay modes occurring close to the limits of stability. The experimental methods used to produce, identify and detect new species and their radiation are discussed. The current theoretical understanding of these decay processes is overviewed. The theoretical description of the most recently discovered and most complex radioactive process - the two-proton radioactivity - is discussed in more detail.

PACS numbers: $23.50 .+z, 23.60 .+e, 23.90 .+w, 23.40 . \mathrm{Hc}$

\section{CONTENTS}

I. INTRODUCTION

A. Radioactivity and Nuclides

B. Brief history

C. Links and connections

1. Nuclear structure

2. Nuclear astrophysics

3. Open quantum systems

D. Outline

\section{LIMITS OF STABILITY}

III. EXPERIMENTAL TECHNIQUES

A. Production

1. Fusion-evaporation

2. Multi-nucleon transfer

3. Fragmentation

4. Spallation

5. Fission

B. Separation

1. In-Flight

* pfutzner@fuw.edu.pl
2. ISOL

3. Future facilities

C. Detection

1. Charged particles

2. Neutrons

3. Signal processing

IV. BETA DELAYED PARTICLE EMISSION

A. Beta decay, general observations

1. The beta strength

2. Delayed particles

B. $\beta^{+}$delayed emission of one particle

1. Occurrence of particle emission

2. Fermi decays

3. Gamow-Teller decays

4. Selected spectroscopic tools

C. $\beta^{-}$delayed emission of one particle

1. Occurrence of particle emission

2. Decays in different mass regions

3. Selected spectroscopic tools

D. Beta delayed emission of several light particles

1. The case of $A=12$

E. Beta delayed fission

V. SINGLE-PROTON RADIOACTIVITY

A. Introduction

1. Fundamentals 
2. Probability of proton emission

3. Spectroscopic factor $S_{p}$

4. Models of proton emission

B. Seniority $s \leq 2$ proton emitters

1. Odd-mass, $s=1$ proton emitters

2. Even-mass, $s=2$ proton emitters

C. Seniority $s>2$ proton emitters

D. Outlook

VI. ALPHA DECAYS

VII. TWO-PROTON RADIOACTIVITY

A. Introduction

1. Two-proton correlations

2. Historical note

B. Experimental results

1. Democratic decays

2. Two-proton emission from excited states

3. Two-proton radioactivity

C. Simplified theoretical models

1. Direct decay model

2. Simultaneous vs. sequential decay

3. Diproton model

D. Three-body model of $2 p$ radioactivity

1. Lifetimes

2. Spatial correlations

3. Momentum correlations and nuclear structure

4. Long-range character of Coulomb interaction in the three-body continuum

E. Two-proton radioactivity and many-body nuclear structure

1. Spectroscopic information in R-matrix approaches

2. Three-body model plus RMF amplitudes

3. Shell model approaches

4. Microscopic cluster models

F. Three and four proton emission

VIII. EMISSION OF NEUTRONS

IX. CONCLUSIONS

Acknowledgments

References

\section{INTRODUCTION}

We consider the atomic nucleus as a quantum object composed of $A$ nucleons (mass number): $Z$ protons (atomic number) and $N$ neutrons, held together mainly by strong nuclear forces. A neutral atom with the specified numbers $A$ and $Z$ is called a nuclide. When using this term, however, we focus on the nuclear component of the atom. Such a system is stable only for certain combinations of numbers $Z$ and $N$. Presently, 256 stable nuclides are known. Systems different from stable configurations undergo spontaneous, radioactive decays until the stability is reached. A nucleus of such an unstable nuclide is considered as a well defined object if its halflife is much longer than $10^{-21} \mathrm{~s}$ which is a characteristic timescale for processes governed by strong interaction. These nuclides are bound by nuclear forces and/or by Coulomb and centrifugal barriers. The number of unstable nuclides synthesized in laboratories is constantly growing, and up to now more than 3000 were identified. In this review, we concentrate on radioactive processes observed for nuclides located at the limits of the nuclear chart. The emphasis is given on new decay processes and features of classical decay modes which do not take place among nuclides close to stability. We will refrain, however, from discussing the heavy frontier of the nuclear chart. The quest for the superheavy elements was reviewed by Hofmann and Münzenberg (2000) and more recently by Oganessian (2007) and by Hofmann (2009a).

\section{A. Radioactivity and Nuclides}

The notion of radioactivity is useful in making distinction between emission of rays or particles by a highly unstable system (for example undergoing a nuclear reaction) from radiation emitted spontaneously by a system whose nuclear and atomic degrees of freedom are close to equilibrium. Such distinction, however, has to be arbitrary and usually a characteristic time scale is used as a criterion. Throughout this review we adopt the following definition. Radioactivity is a process of emission of particles by an atomic nucleus which occurs with characteristic time (half-life) much longer than the K-shell vacancy half-life in a carbon atom, which amounts to about $2 \times 10^{-14} \mathrm{~s}$ (Bambynek et al. 1972). A relativistic particle travels in the time of $10^{-14} \mathrm{~s}$ a distance of a few micrometers which is close to the measurement limit in a nuclear emulsion. In addition, this value coincides with a decay width, defined as $\Gamma=\ln 2 \hbar / T_{1 / 2}$, of about $0.03 \mathrm{eV}$ which is roughly the thermal energy at room temperature. Thus, nuclear processes much slower than filling the K-vacancy, whose duration in principle can be measured directly, and with the width much smaller than the thermal energy at room temperature will be called radioactive. This definition applies to both nuclear ground 


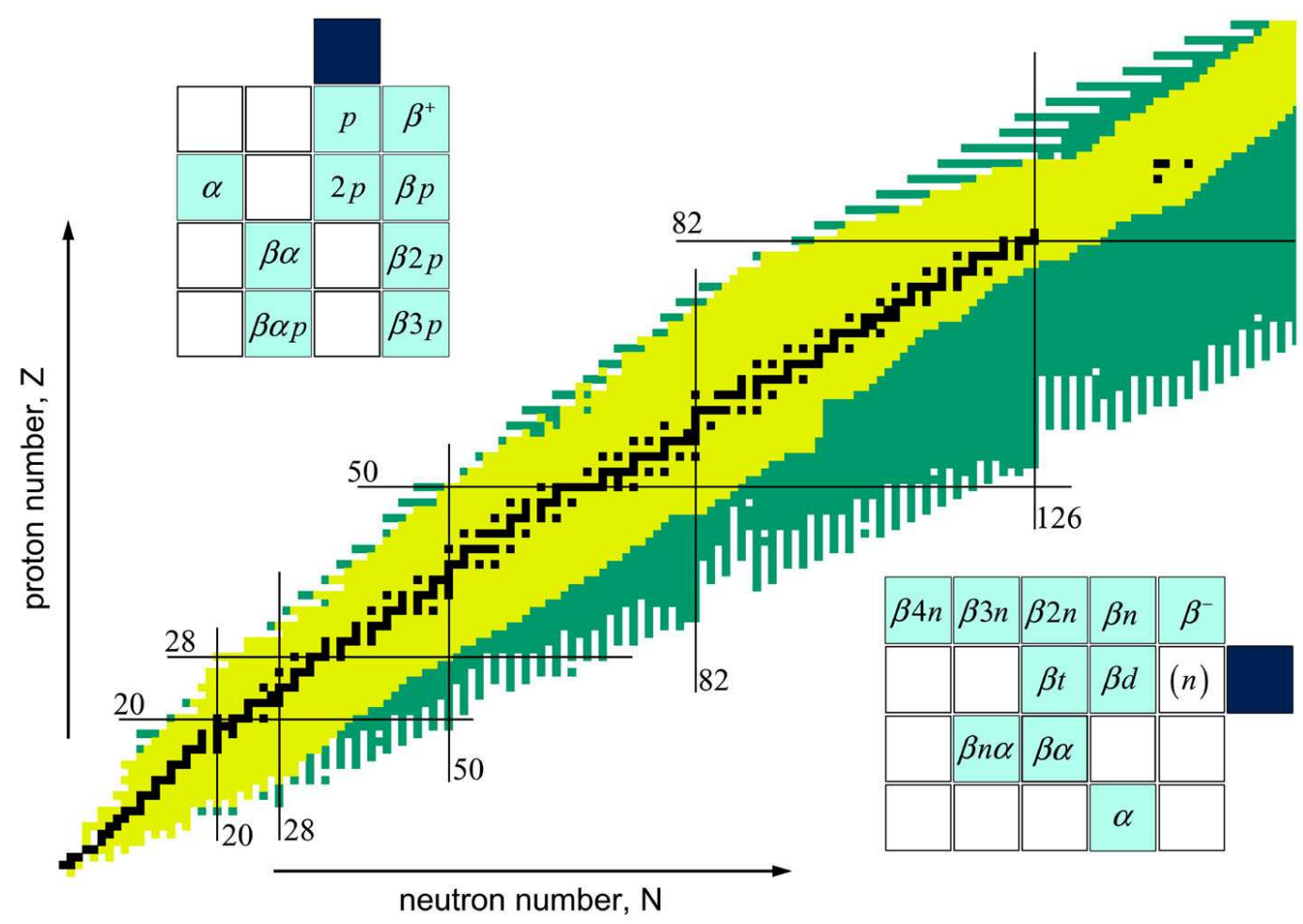

FIG. 1 (Color online) The chart of nuclei. The stable nuclides and the radioactive ones which were experimentally identified are shown by black and light/yellow, respectively. The nuclides predicted to have positive nucleon separation energy according to the FRDM mass model (Möller et al., 1997), but not yet observed, are shown in dark/green. The lines indicate position of magic numbers corresponding to the closed neutron and proton shells (the numbers smaller than 20 are not shown). The insets show the location on the chart of the decay products of the parent nucleus which is indicated by a dark square. The observed decay channels of the proton-rich and the neutron-rich nuclei are shown on the left and on the right inset, respectively.

states and to long-lived excited nuclear states (isomers).

The definition of a nuclide relates to the definition of radioactivity. A nuclide is a neutral atom, specified by the numbers $A$ and $Z$ of its nucleus, which is either stable or lives long enough to be classified as radioactive. We say that a nuclide does not "exist" if its nucleus decays too fast to be called radioactive. All existing nuclides are represented on a chart of nuclides spanned by the atomic number $Z$ and neutron number $N$ (Figure 1 ). In the last three decades their number was growing almost steadily from about 2200 in 1981 to about 3000 in 2006 (Pfennig et al. 2008), giving an average of about 30 new nuclides identified per year. Due to vigorous growth of nuclear facilities (Sec. III.B) this trend is expected to continue in next decades.

The domain of processes occurring on a time scale shorter than radioactivity is referred to as the resonant regime. The resonant phenomena are characterized by features having a directly measurable width in the energy spectra. Typically, widths of order meV is taken as the lower end of the resonant regime. Thus, the distinction between radioactive and resonant phenomena is that the former have a characteristic time which can be measured directly while the latter have a characteristic energy width which can be measured directly.
Characteristic time scales for different radioactive decays are illustrated schematically in Figure 2

\section{B. Brief history}

The discovery of radioactivity by Becquerel (1896) and the subsequent discovery of new radioactive elements, polonium (Curie and Curie, 1898) and radium (Curie et al. 1898) initiated a scientific breakthrough into the world of subatomic structure of matter. Through early works on $\alpha$ - and $\beta$-rays, distinguished and named by Rutherford (1899) and on $\gamma$-radiation, discovered by Villard (1900), a manifold of nuclear phenomena started to emerge. The nature of the new rays was soon clarified (Rutherford and Geiger, 1908), (Kaufmann, 1902), (Rutherford and da C. Andrade, 1914). Finally, two other milestones: the discovery of the atomic nucleus (Rutherford, 1911) and of the neutron (Chadwick, 1932) gave birth to a new discipline - nuclear physics. A brilliant and detailed account of these early years was given by Pais (1986).

The full understanding of decay processes required quantum mechanics, as an illustrious explanation of $\alpha$ decay by Gamow (1928) and Gurney and Condon (1928) 


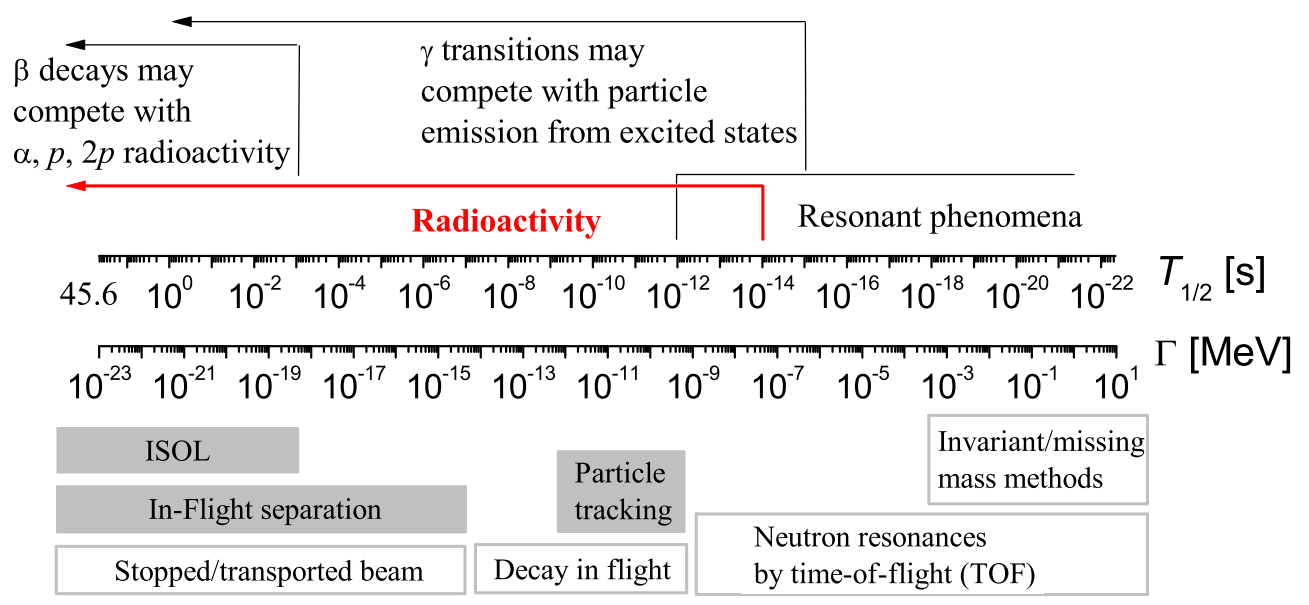

FIG. 2 Characteristic time scales and decay widths of radioactive decays. Ranges of application of selected experimental techniques are sketched.

demonstrated. Soon afterwards, early ideas of quantum field theory led Fermi to formulate the first theory of $\beta$ decay (Fermi, 1934). A novel type of radioactivity - the $\beta^{+}$decay — was discovered by Curie and Joliot (1934). It was also the first instant of a radioactive nuclide synthesized in a laboratory in contrast to all previously known radioactive substances of natural origin. Wick (1934), and independently Bethe and Peierls (1934), realized that a process of orbital electron capture (EC) by an atomic nucleus is possible. Thus, the EC decay was the first type of radioactivity predicted theoretically. It was confirmed by an observation of K-capture by Alvarez (1937). Spontaneous fission, discovered by Flerov and Petrzhak (1940), completed the list of "classical" radioactive decay modes. Ever since, they have played a crucial role in learning about nuclear properties, in tagging reaction channels, and in identifying new nuclides.

The beginning of the modern era of radioactivity can be marked by the work of Goldansky (1960) who in a systematical study considered properties of very neutron deficient nuclei and discussed possible decay modes like $\beta$-delayed proton emission and proton radioactivity. He was the first to point out the possibility of the two-proton radioactivity and to describe its key features. The first emission of a proton following $\beta$ decay was reported by Karnaukhov et al. (1963). The first emission from an identified precursor was observed from an excited state of ${ }^{25} \mathrm{Al}$ populated in the $\beta^{+}$decay of ${ }^{25} \mathrm{Si}$ (Barton et al. 1963). Such a variant of $\beta$ decay - the $\beta$-delayed emission of particles from states of a daughter nucleus become a field of study in its own and includes various decay channels with emission of protons, neutrons, $\alpha$ particles, deuterons, and tritons, see Figure1. This is the subject of Sec. IV] The first direct emission of a proton from a nuclear state was observed to proceed from an isomeric state in ${ }^{53} \mathrm{Co}$ (Jackson et al., 1970$)$. The ground- state proton radioactivity was observed for the first time in ${ }^{151} \mathrm{Lu}$ (Hofmann et al., 1982 ) and in ${ }^{147} \mathrm{Tm}$ (Klepper et al. 1982). Since then, almost fifty proton emitters, including emission from isomeric states, were identified. This field is covered in Sec. V The prediction of the twoproton radioactivity $(2 p)$ had to wait much longer for experimental confirmation. Such a decay mode was observed first for ${ }^{45} \mathrm{Fe}$ by Pfützner et al. (2002) and in an independent experiment by Giovinazzo et al. (2002). This freshly opened field of nuclear spectroscopy is covered in Sec. VII

For completeness, one should mention the cluster radioactivity, in which a nuclear fragment heavier than the $\alpha$-particle but lighter than fission fragments, is emitted. This decay mode was discovered by Rose and Jones (1984) who identified ${ }^{14} \mathrm{C}$ ions emitted by ${ }^{223}$ Ra. Later, many similar decay channels were observed (Bonetti and Guglielmetti, 2007; Poenaru et al. 2002). In all of them, however, the dominant decay mode is $\alpha$ decay, the cluster emission being less probable by a factor of at least $10^{9}$. In addition, this rare decay mode can be established only in long-living nuclei, not far from the nuclear stability. Thus, it is beyond the scope of this paper.

\section{Links and connections}

In general, information on nuclei come from different and complementary experimental approaches. Radioactive decay always was one of them and still remains a major tool in nuclear physics as well as in numerous branches of physics where nuclear degrees of freedom are relevant. An important attribute of the decay is the characteristic half-life. The very delay between the moment of production and the decay event, offers a filtering possibility, thus helping to increase the signal-to-background ratio. The 
values of the half-life provide a way to identify processes and offer tests to nuclear models. Another important facet of spontaneous decays are selection rules, which reflect conservation laws obeyed by underlying interactions. Effectively, they provide different filtering mechanisms, essential for the planning of measurements and for interpretation of their results.

The quest for superheavy elements may exemplify the role of radioactive decays (Hofmann and Münzenberg, 2000). The emission of $\alpha$ particles by synthesized heavy nuclides provides the very proof of their existence and a clean way for their identification. Moreover, the $\alpha$ decay is a powerful marker helping to establish the first chemical properties of a few-atom sample of a new element, like in the case of ${ }^{283} \mathrm{Cn}$ (Eichler et al. 2007).

Radioactivity plays a significant role in fundamental research, as can be illustrated by historical examples of the neutrino hypothesis by Pauli (1930) and the discovery of parity non-conservation in the $\beta$ decay of ${ }^{60} \mathrm{Co}(\mathrm{Wu}$ et al., 1957). A recent review of tests of the standard electroweak model by the beta decay (Severijns et al. 2006) gives a comprehensive summary of this field.

However, since the present paper is limited to radioactive studies at the limits of stability, below we mention briefly a few research areas where they are particularly important.

\section{Nuclear structure}

With increasing scope of studies on the chart of nuclei new patterns emerge and novel features are predicted. An observed anomaly in masses of neutron-rich sodium isotopes (Thibault et al. 1975) is an early example which led to the notion of an "island of inversion" in vicinity of ${ }^{32} \mathrm{Mg}$ resulting from changes in sequence of single-particle orbitals (Nummela et al. 2001; Yordanov et al., 2007). Recently, a similar phenomenon was claimed to occur around ${ }^{62} \mathrm{Ti}$ (Flanagan et al. 2009, Tarasov et al., 2009). It was realized that the classical shell gaps - the cornerstones of microscopic description of nuclei - do migrate in areas distant from stability. A detailed survey of the present situation is given by Sorlin and Porquet (2008). A new feature of quenching of the shell-gaps due to influence of continuum states was predicted close to the neutron drip line (Dobaczewski et al. 1994). A comprehensive review of novel aspects of nuclear structure emerging far from the beta stability is given by Dobaczewski et al. (2007).

The experimental reach for most exotic nuclides suffers from very low production yields. Radioactive decays allow not merely to identify a system of interest but often offer the only practical source of structural information. The first half-life determination for the doubly magic ${ }^{78} \mathrm{Ni}$ was done with 11 atoms (Hosmer et al., 2005). The first insight into the structure of ${ }^{45} \mathrm{Fe}$ resulted from decays of 75 atoms (Miernik et al. 2007c). The first information on excited states in ${ }^{70} \mathrm{Ni}$ was deduced from a few tens of counts in a singles gamma spectrum (Grzywacz et al. , 1998). Here, the very clean selection was provided by a delayed decay of a microsecond isomer. Such first information, in turn, becomes essential in later, more advanced experiments, allowing for coincidence measurements or for selection of events in high-background conditions, for example, by decay tagging (Jenkins et al., 2000, Seweryniak et al. 1997).

Recently, the insights gained on nuclear structure near the proton drip-line by means of radioactive decay studies were reviewed by Blank and Borge (2008).

\section{Nuclear astrophysics}

Several subjects of modern astrophysics are related to properties of atomic nuclei. They include questions on the origin of the elements, physics of compact objects like neutron stars or white dwarfs, studies of stellar explosions like supernovae, x-ray bursts, and many others. Although the most frequently requested nuclear data in astrophysics are reaction cross sections for some key processes, the radioactive decays provide indispensable information for many applications. Nuclei very far from the beta stability play a particularly important role in nucleosynthesis of elements heavier than iron, as pointed out in the seminal paper of Burbidge et al. (1957). The rapid neutron capture ( $r$-process), responsible for about half of the abundance of elements above iron, including all of uranium and thorium, passes through regions of very neutron-rich nuclei, mostly far beyond the reach of present experiments (Kratz et al., 2007). In turn, a number of neutron-deficient isotopes was produced in the rapid proton capture ( $r p$ - process) which involves very neutron-deficient areas on chart of nuclei (Schatz et al. 1998). Both processes are expected to occur in explosive stellar conditions, details of which are still under debate. Theoretical reconstruction of these processes is additionally hindered by the lack of relevant nuclear data. To those of special interest belong masses, half-lives, branching ratios, and beta-delayed particle emission probabilities. In principle, these values can be determined from radioactive decay studies. Presently, however, most of the nuclei important in this context are either difficult or impossible to synthesize in reasonable quantities. The theoretical models or empirical parameterizations have to be relied on, instead. Thus, the motivation to extend experimental decay studies to the limits of nuclear stability is twofold. They deliver data directly needed by astrophysical calculations, and they help to test and improve models of nuclear structure, increasing reliability of theoretical extrapolations.

A thorough and detailed discussion of the present status of the interplay between nuclear structure and as- 
trophysics, with the emphasis on stellar evolution and nucleosynthesis, can be found in Langanke and MartínezPinedo (2003) and in Grawe et al. (2007). Both these reviews reveal the necessity of exploring exotic nuclei and their decay properties in order to fully understand astrophysical aspects of our Universe.

\section{Open quantum systems}

Several fields of modern physics face the goal of describing quantum many-body systems which are not isolated from its quantal environments. Examples of such open quantum systems include quantum dots, droplets of neutral atoms, microwave cavities, or weakly bound nuclei very far from the beta stability (Okołowicz et al. 2003). Nuclear physics at the limits of stability appears as a particularly promising testing ground of new concepts. When the nuclear binding energy decreases, the conceptual separation of well localized, bound states from the continuum scattering states becomes artificial and, in fact, is hampering the correct description of various features, like neutron halos, Thomas-Ehrman shifts, or clustering phenomena (Dobaczewski et al., 2007, Michel et al. 2010a).

The quest to formulate a unified description of nuclear structure and nuclear reactions resulted in extensions of nuclear shell model, like the Shell Model Embedded in the Continuum (Bennaceur et al. 1998; Okołowicz et al. 2003), or more recently the Gamow Shell Model (Michel et al. 2002, 2009). New spectroscopic data on very exotic nuclei, and on complex decay modes, like two-proton radioactivity, or beta-delayed multi-particle emission would stimulate further developments in this field. Thus, radioactive decays at the limits of nuclear stability may be instrumental in improving our fundamental understanding of many-body quantum systems.

\section{Outline}

In Section II we discuss the limits of nuclear stability using the concept of the drip-line based on nucleon separation energy. The experimental situation in accessing both the proton and the neutron drip-line is briefly presented. The experimental techniques pertaining to radioactivity studies far form stability are reviewed in Section III. Various reactions used to produce exotic nuclides and the main methods of their separation are shortly described. Finally, selected detection techniques of special importance for measurements of radioactive decays are presented. The following Sections IV VII are devoted to the main radioactive decay modes at the limits of stability: $\beta$-delayed particle emission, proton radioactivity, $\alpha$ emission, and two-proton radioactivity. The latter decay mode is treated in considerably more detailed way, as it is the least known and its understanding is still in a status of development. In Section VIII the prospects of neutron radioactivity are examined. The main conclusions of the paper are shortly summarized in the final Section IX.

Throughout this work we use the system of units in which $\hbar=c=1$. 


\section{LIMITS OF STABILITY}

The limits of the nuclear world are determined by the nuclear binding energies. The limits relevant to this review are often characterized by the drip lines which separate bound systems from the unbound ones. Although different definitions can be encountered in the literature, we adhere to the simplest and most common one which is based on the single-nucleon separation energy. The proton- and the neutron separation energy of a nuclide with numbers $N$ and $Z$ are given by:

$$
\begin{gathered}
S_{p}(N, Z)=B(N, Z)-B(N, Z-1) \\
S_{n}(N, Z)=B(N, Z)-B(N-1, Z) .
\end{gathered}
$$

The $B(N, Z)$ is the binding energy of the nuclide related to its mass $M(N, Z)$ :

$$
M(N, Z)=Z M_{H}+N m_{n}-B(N, Z),
$$

where $M_{H}$ and $m_{n}$ are masses of the hydrogen atom and the neutron, respectively.

When we move along the line of isotopes with the given atomic number $Z$, starting from stability towards neutron-deficient nuclides, the proton separation energy $S_{p}$ decreases and at certain location it becomes negative. The proton drip-line is defined as the border between the last proton-bound isotope and the first one with the negative value of the $S_{p}$. The typical situation, according to the predictions of a particular mass model (Möller et al., 1997) for the isotopes of iron and cobalt is presented in Figure 3. It follows from this model that the proton dripline for iron should lie between ${ }_{26}^{45} \mathrm{Fe}_{19}$ and ${ }_{26}^{46} \mathrm{Fe}_{20}$, while in case of cobalt it is located between ${ }_{27}^{49} \mathrm{Co}_{22}$ and ${ }_{27}^{50} \mathrm{Co}_{23}$. Generally, the proton drip-line for odd $Z$ isotopes is closer to stability than in case of the neighboring even- $Z$ which results from the proton pairing energy.

In the fully analogous way, the neutron drip-line for a given neutron number $N$ is defined as a border between the last neutron bound isotone, when counting from stability, and the first one for which the neutron separation energy $S_{n}$ is negative. The predicted separation energies for the $N=26$ and $N=27$ isotones are shown in Figure 4. Thus, for $N=26$ the neutron drip-line is expected to lie between ${ }_{9}^{35} \mathrm{~F}_{26}$ and ${ }_{10}^{36} \mathrm{Ne}_{26}$. Similarly to the proton case, the neutron drip-line for the odd- $N$ is closer to stability than for the neighboring even- $N$ which reflects the neutron pairing energy.

The drip lines as defined above are very useful in identifying and discussing limits of stability, but to some extend they are arbitrary and they do not provide the unambiguous demarkation of nuclear stability. This can be seen by inspecting the two-nucleon separation energies:

$$
\begin{gathered}
S_{2 p}(N, Z)=B(N, Z)-B(N, Z-2) \\
S_{2 n}(N, Z)=B(N, Z)-B(N-2, Z) .
\end{gathered}
$$

Due to the pairing interaction, in case of an even number of nucleons the two-nucleon separation energy can
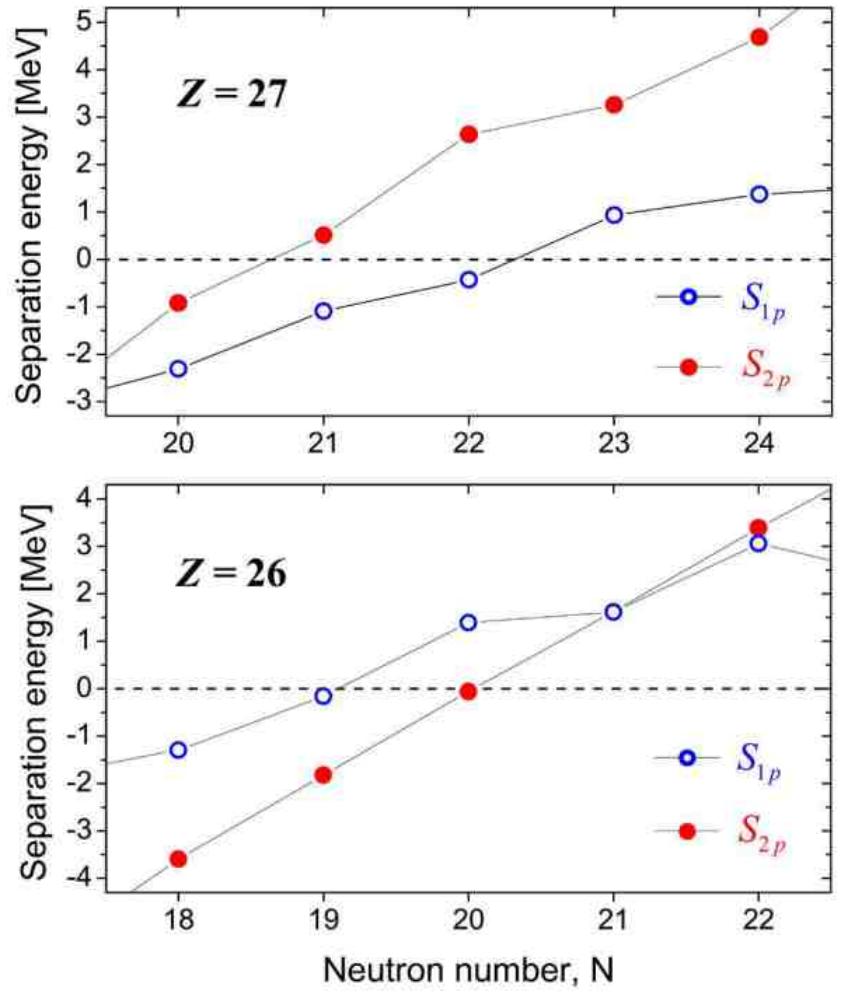

FIG. 3 (Color online) The proton- and two-proton separation energies of iron and cobalt isotopes as predicted by the FRDM mass model (Möller et al. 1997).

be smaller than the single-nucleon value. For the cases discussed above, it is illustrated in Figures 3 and 4 Although the proton separation energy in ${ }_{26}^{46} \mathrm{Fe}_{20}$ is positive, this nuclide is expected to be slightly two-proton unbound. Similar situation is observed in the $N=26$ isotones - the two-neutron instability develops first, before the neutron drip-line is reached. The additional complication, which is essential on the neutron-deficient side, comes from the fact that the exact position of a nuclide with respect to the drip-line cannot determine alone its dominant decay mode. This is caused by the Coulomb and centrifugal barriers which hamper emission of nucleons. Only when the nucleon penetration probability through the barrier, depending on the energy and the angular momentum of the initial state, is large enough, the particle radioactivity can compete with $\beta$ decay. Thus, although the mentioned ${ }_{26}^{46} \mathrm{Fe}_{20}$ may be twoproton unbound, it is known to decay by $\beta^{+}$transition. In turn, ${ }_{26}^{45} \mathrm{Fe}_{19}$ is sufficiently two-proton unbound to decay predominantly by the $2 p$ radioactivity, although the $\beta^{+}$channel has a substantial branching (Miernik et al. 2009). The exact position of this nucleus with respect to the proton drip-line turns out to be irrelevant for its radioactive decay. In case of odd- $Z$ nuclides, the proton radioactivity can win the competition with $\beta$ decay only when its proton separation energy is sufficiently negative. 

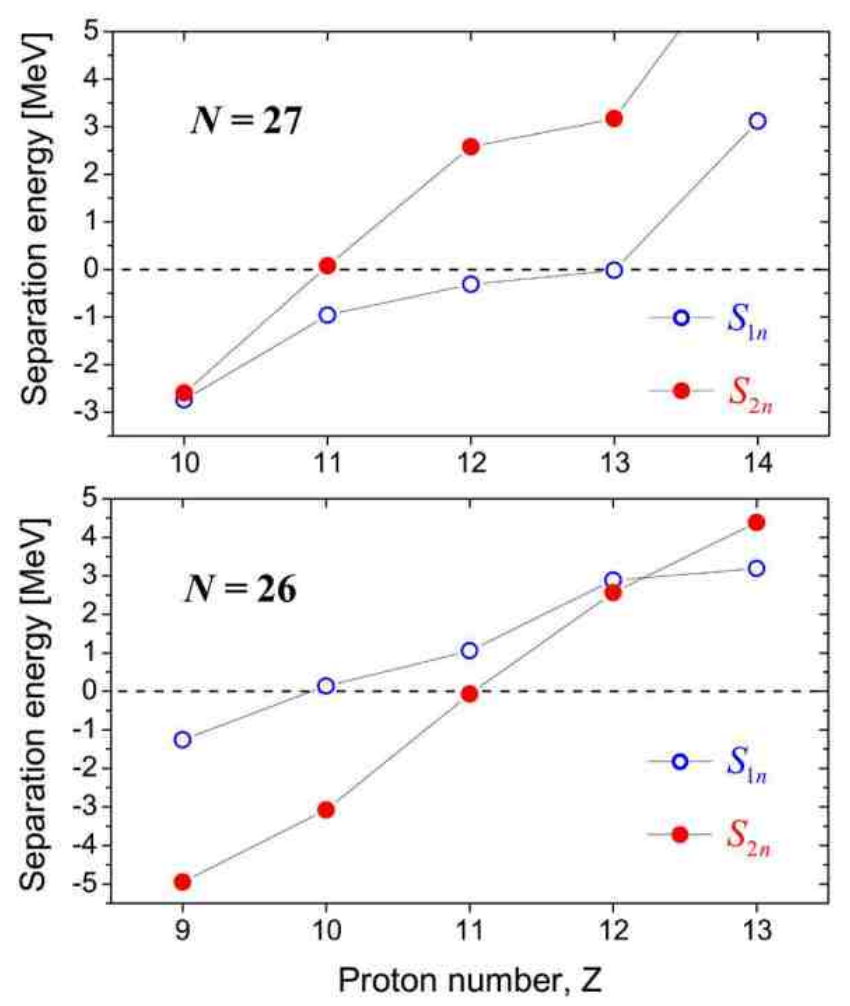

FIG. 4 (Color online) The neutron- and two-neutron separation energies for the $N=26$ and $N=27$ isotones, as predicted by the FRDM mass model (Möller et al. 1997).

Thus, the observation of the proton radioactivity proves that the nuclide is located beyond the proton drip-line but the exact position of this line cannot be determined from decay data alone. This can be achieved only by precise mass measurements of nuclides in the region of interest.

We note, that on the neutron-deficient edge of the chart of nuclides above tungsten the dominant decay mode is the $\alpha$ emission which happens to proceed faster than $\beta$ decay. Thus, beyond the proton drip-line in this region, the proton radioactivity competes actually with $\alpha$ decay.

At the neutron drip-line the situation is different because the unbound neutrons are not affected by the Coulomb barrier. The influence of the centrifugal potential alone is much weaker as it decreases with radius effectively as $1 / r^{2}$ in contrast to the $1 / r$ dependence of the Coulomb potential. In consequence, the effect of the centrifugal barrier is expected to be observable only in rare cases of very low decay energies and large angular momentum. The resulting possible neutron and two-neutron radioactivity is examined in more detail in Sec. VIII. For practical purposes, any system with negative neutron- or two-neutron separation energy can be expected to live too short to be qualified as radioactive. Therefore, the limits of stability on the neutron-rich side could in prin-

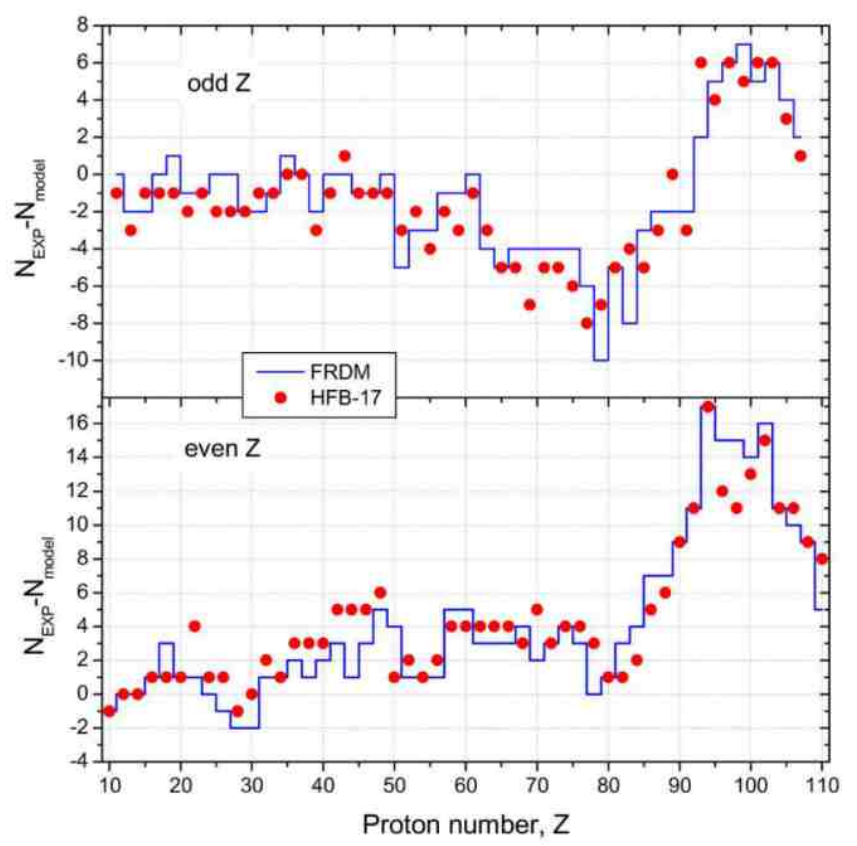

FIG. 5 (Color online) The difference between the neutron number of the lightest experimentally observed isotope for a given atomic number $Z$ and the corresponding prediction for the last isotope before the proton drip-line according to the FRDM mass model (Möller et al. 1997)(line) and the HFB17 model (Goriely et al. 2009) (circles). The results for the even $Z$ and the odd $Z$ are shown in the bottom and in the top, respectively. The experimental values were taken from Magill et al. (2009) with corrections contained in Baumann et al. (2007).

ciple be established rather precisely by inspecting which is the lightest isotone still undergoing radioactive decay. The problem, however, is that it is very difficult to reach experimentally the neutron drip-line for $N>28$.

The general picture is presented in Figure 1 where all nuclides identified experimentally until now are superimposed on the plot of all nuclides predicted to have positive proton and neutron separation energy by the Finite Range Droplet Model (FRDM) developed by Möller et al. (1995) and Möller et al. (1997). This model is a successful representant of a class of macroscopic-microscopic mass formulae, combining the macroscopic liquid-drop parametrization with the microscopic shell and pairing corrections. The prediction reveals a few characteristic features, like the even-odd staggering for the neutron deficient isotopes and for the proton deficient isotones, or the strong influence of the $N=82$ and $N=126$ shells on the neutron-rich side of the chart. To illustrate theoretical uncertainties we compare predictions of the FRDM model with results of the HFB-17 model (Goriely et al. 2009) which represents a class of fully microscopic approaches based on Hartree-Fock-Bogoliubov formalism and Skyrme forces (Goriely et al. 2010 ). Figure 5 presents the current experimental situation and the com- 


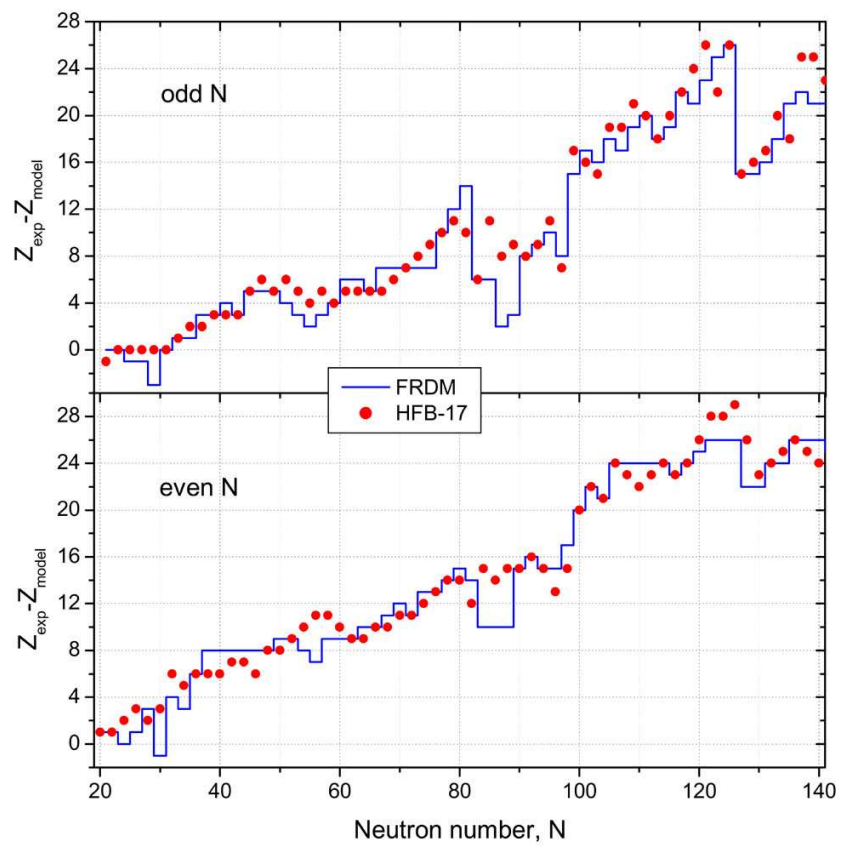

FIG. 6 (Color online) The difference between the proton number of the lightest observed isotone for a given neutron number $N$ and the prediction of the last stable isotone before the neutron drip-line according to the two theoretical mass models. The results for the even $N$ and the odd $N$ are shown in the bottom and in the top, respectively. The plot details are the same as in Figure 5.

parison of the two models. It shows differences between the neutron number of the lightest observed isotope and the predicted values for the last proton-stable isotope before the proton drip-line. We see that both predictions agree well with each other - they follow the same pattern and they differ by a few units at most. The negative values indicate those observed nuclides which are located beyond the predicted proton-drip line, in most cases they are proton emitters. The large group of such nuclides, seen for odd- $Z$ values between 50 and 90 illustrates the strong impact of the Coulomb barrier on the heavy nuclei. On the other hand for almost all even- $Z$ elements, there are predicted bound isotopes which remain to be observed. A distinguished peak of positive values for the $Z>90$ results from experimental difficulties to produce proton-rich nuclei in this region.

The analogous information for the neutron-rich side is given in Figure 6 where the differences between the proton number of the lightest observed isotone and the predicted values for the last neutron-stable isotone before the neutron drip-line are plotted. Again, both models are consistent with each other. The largest difference is seen above the $N=80$ where the FRDM model seems to exhibit large variations due to the neutron shell closure an effect not pronounced in the HFB-17 model. In contrast to the proton-rich side, however, almost all values are positive and they increase rapidly with the increasing neutron number. This reflects the fact that except for the light nuclei, the neutron drip-line is far from the body of presently observed nuclides, as is dramatically evident also in Figure 1. In fact, the drip-line has been determined experimentally and unambiguously for even $N$ only up to $N=20$ and for odd $N$ up to $N=27$ (Thoennessen, 2004).

The significant expansion of the body of observed nuclides, especially on the neutron-rich side of the chart, is expected only when the next generation of radioactive beam facilities will come into operation, see Sec. III.

The current status of the knowledge on atomic masses and of the global mass models can be found in Lunney et al. (2003). The detailed discussion of the present experimental knowledge of the limits of nuclear stability was presented by Thoennessen (2004). 


\section{EXPERIMENTAL TECHNIQUES}

Experimental studies of nuclei at the limits of stability belong to the front-line of physical research. A view on experimental techniques, given in this section, provides a general perspective on the advanced methods of present-day low-energy nuclear physics. First, reactions used to produce radioactive nuclides will be mentioned followed by a short description of the main methods of their extraction and separation. Then, selected aspects of modern detection systems will be reviewed with an emphasis on recording manifestations of radioactivity.

\section{A. Production}

The methods of production of nuclides far from beta stability are almost exclusively based on nuclear reactions involving stable nuclides or their ions. In a simplified view, a new nucleus is formed either by fusion of two other nuclei (projectile and target), by exchange of nucleons between the projectile and the target nuclei (transfer), or in reaction leading to removal of nucleons either from the target or from the projectile nucleus (fragmentation, spallation, fission).

In principle, a radioactive nucleus produced in one of these reaction, having sufficiently long half-life, can be used as a projectile to initiate a secondary reaction in which nuclei even further from stability are formed. This is the idea of radioactive beams which has been driving many experimental developments (JPG, 2011; Tanihata, 2008). It is anticipated that reactions induced by radioactive beams will play a major role in the future expansion of the chart of nuclei. In addition, the secondary reactions induced by radioactive projectiles represent one of the main methods to produce radioactive nuclides with the shortest half-lives, in the nanosecond range or shorter. For completeness, we note that in some cases a radioactive target may be used for the production of exotic nuclei. For example, in the recent discovery of a new element with the atomic number $Z=117$, the radioactive target of ${ }^{249} \mathrm{Bk}$ was used Oganessian et al. 2010).

Each production method has its own characteristics and a typical application range. In the following we mention briefly various reactions which are being used and we direct the reader to papers providing more detailed and broader presentations, as well as references to relevant technical contributions. Some aspects of reactions used to produce exotic nuclei were discussed by Geissel et al. (1995) and more recently by Schmidt et al. (2002).

\section{Fusion-evaporation}

In a central collision at low energy two nuclei can join together (fusion) to form a single heavier nucleus. In the second step, the resulting compound nucleus releases its excitation energy by emission of nucleons (evaporation) and radiation. The reaction cross section is very sensitive to the initial energy in the projectile-target system, which must be close to the Coulomb barrier. If the energy is too low, the probability of barrier penetration drops dramatically, if it is too large, other channels start to dominate. This is the key reaction in the synthesis of superheavy elements (Hofmann, 2009b; Schmidt and Morawek. 1991). However, since the final nucleus tends to be located on the neutron-deficient side of stability, the fusionevaporation is successfully used to produce very neutrondeficient systems. In fact, most of research on proton radioactivity employs this reaction (Ferreira and Arumugan, 2007; Woods and Davis, 1997). The most commonly used tool for optimizing experimental conditions and for prediction of cross-sections is the statistical code HIVAP (Reisdorf, 1981, Reisdorf and Schädel, 1992), but other statistical codes like CASCADE (Pühlhofer, 1977) or PACE (Gavron, 1980) are also being used. The potential of the fusion-evaporation reaction can be illustrated by an attempt to reach $\alpha$-emitters above ${ }^{100} \mathrm{Sn}$ (Korgul et al. 2008) and by an investigation to produce the lightest isotopes of bismuth and polonium Andreyev et al. 2005).

\section{Multi-nucleon transfer}

Transfer reactions belong to the category of binary processes where instead of a fused system of two heavy ions a projectile-like and a target-like nucleus appear in the final state. This happens if the collision is not central. If it is also deep-inelastic (damped), a few nucleons can be exchanged between reaction partners leading to radioactive products. Although a part of the energy of the relative motion goes into the excitation of the final fragments, which is released by evaporation of light particles, still residual nuclei far from stability can be formed. Such multi-nucleon transfer reactions at Coulomb barrier energies has been used to produce unstable nuclides, including neutron rich ones (Broda, 2006). The method is mainly used in combination with in-beam $\gamma$-ray spectroscopy and isomeric spectroscopy in various regions of the chart on nuclei (Cocks et al., 2000, Montanari et al. 2011). The main advances on this field in the last decade and the summary of theoretical understanding of the reaction mechanism are given in the recent review by Corradi et al. (2009). The current limits of nuclear stability cannot be reached by multi-nucleon transfer between stable projectile and target, but the importance of this reaction is increasing with developments of radioactive 
beams. The transfer reactions are considered also as a tool to produce new isotopes in the region of superheavy nuclei (Zagrebaev and Greiner, 2008).

\section{Fragmentation}

When the collision energy of two heavy nuclei is large compared to the Fermi energy of nucleons, the probability that nucleons will be exchanged between the reaction partners becomes very small. Instead, violent interactions occur in the overlapping zone of the projectile and the target (participants), while their parts outside this zone (spectators) emanate as the projectile-, and target-like prefragments, respectively. After this abrasion phase, the cooling of prefragments by evaporation of particles, by radiation, or by fission proceeds and the final fragments are formed. If the excitation energy of the prefragment is large, which happens in more central collisions, the multifragmentation takes place, i.e. the break-up into many intermediate-mass fragments. In the so called limiting fragmentation regime, for projectile energies above $100 \mathrm{MeV} /$ nucleon, a characteristic feature is observed that the total reaction cross section weakly depends on projectile energy and can be approximated by a simple geometric formula

$$
\sigma_{R}=\pi r_{0}^{2}\left(A_{T}^{1 / 3}+A_{P}^{1 / 3}-c\right),
$$

where $A_{T}$ and $A_{P}$ are the mass numbers of the target and the projectile, respectively, the radius parameter is $r_{0}=1.1 \mathrm{fm}$, and a correction for nuclear transparency is introduced by a parameter $c \cong 2$ (Kox et al., 1987$)$.

In the present context, the fragmentation of the projectile plays a special role. When high energy projectile ions collide with target nuclei, the projectile-like fragments surviving the abrasion phase continue moving with almost no change of velocity. Thus, the resulting unstable nuclei form a secondary beam which can be transported and filtered by means of ion-optical devices. This method is very fast and universal, since practically any nucleus with numbers $N$ and $Z$ smaller than those of the projectile can be produced. These features make fragmentation one of the key reactions for radioactive beam facilities. The method of projectile fragmentation was pioneered at Bevalac facility at the Lawrence Berkeley Laboratory (Symons et al., 1979, Westfall et al., 1979). Later the systematic studies of fragmentation cross sections as a function of energy, projectile, and target were carried out by Webber et al. (1990). Recently, comprehensive studies of projectile-like fragmentation are being carried out at SIS-FRS system at GSI Darmstadt (Benlliure et al., 2008, Henzlova et al., 2008). The most advanced theoretical description of the fragmentation is currently achieved in a modern version of the abrasionablation model and is implemented as the Monte-Carlo code ABRABLA (Gaimard and Schmidt, 1991). The mechanism of prefragment excitation is understood in this model as a result of random creation of holes in the nucleonic Fermi distribution (Schmidt et al. 1993$)$. The evaporation stage is modeled with the code ABLA ( $\mathrm{Ke}-$ lić et al. 2008). The simpler, analytical version COFRA (Benlliure et al. 1999, 2000) is applicable to the veryneutron rich fragments (cold fragmentation). For practical estimates, the empirical parametrization of the fragmentation cross sections is given by a simple analytical model EPAX (Sümmerer and Blank, 2000).

\section{Spallation}

If in a high energy collision (above $100 \mathrm{MeV} /$ nucleon) one of the reaction partners is a light ion, like proton, deuteron, or triton, the process is referred to as spallation. From the perspective of production of exotic nuclei, the main difference from projectile fragmentation is that in case of spallation usually the target nucleus being the heavier partner is the source of radioactive nuclei. In addition, the mechanism of its primary excitation is different. The first step of the spallation is usually described as a series of collisions between nucleons in the target nucleus, induced by the projectile, which forms the base of intranuclear cascade models (INC). Such a cascade of collisions leads to a highly excited system which, in the second phase, deexcites in the same way as the hot target-like prefragment. In consequence, the target nucleus is destroyed, and in analogy to the fragmentation, practically any nucleus with numbers $N$ and $Z$ smaller than those of the target can be produced. Due to this universality, the spallation is the second main production process considered for radioactive beam facilities. In contrast to fragmentation, however, the radioactive products have to be extracted from the target material.

The example of a modern version of an INC approach with a discussion of the physics involved is given by Boudard et al. (2002). For the second stage of the reaction, various versions of statistical evaporation codes are being used (Le Gentil et al., 2008). The detailed experimental studies of the spallation are conveniently performed in the inverse kinematics, where a light target (hydrogen, deuterium) is bombarded by heavy nuclei accelerated to relativistic energy. Such studies are performed with use of the fragmentation facility which reflects a symmetry between these two reactions. This method was recently used at GSI Darmstadt in a comprehensive study of residual fragments produced by the spallation of ${ }^{238} \mathrm{U}$ by protons (Ricciardi et al. 2006 , Taieb et al. , 2003) and by deuterons (Casarejos et al. 2006; Pereira et al. , 2007). A similar work on spallation of ${ }^{136} \mathrm{Xe}$ and ${ }^{56} \mathrm{Fe}$ by protons was reported by Napolitani et al. (2007) and by Le Gentil et al. (2008), respectively. 


\section{Residues of ${ }^{208} \mathrm{~Pb}+\mathrm{x}$ and ${ }^{238} \mathrm{U}+\mathrm{x}$ at $1 \mathrm{~A} \mathrm{GeV}$}

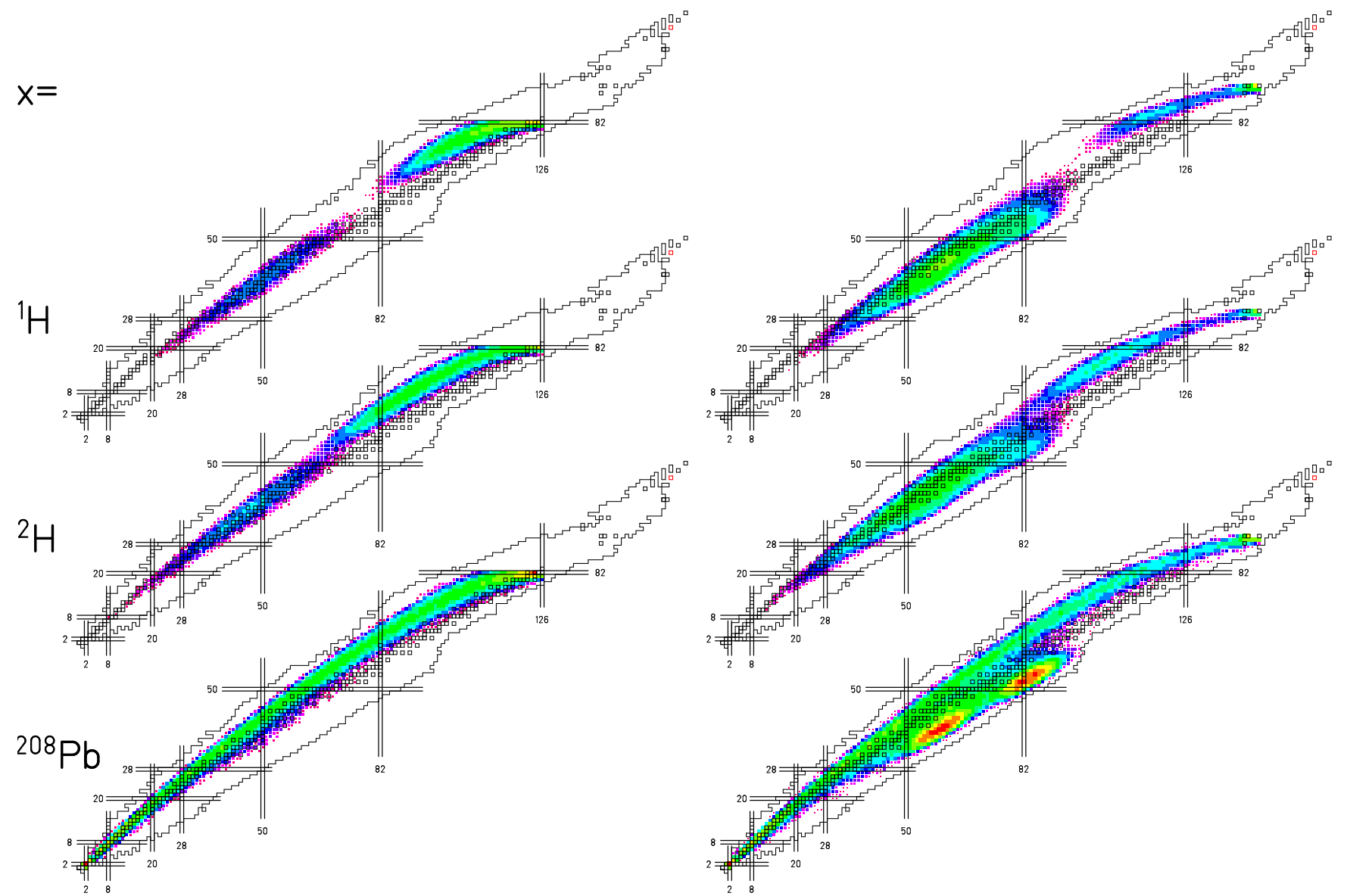

FIG. 7 (Color online) Systematic overview on calculated isotopic production cross sections in different reactions. For clarity only values above $100 \mu \mathrm{b}$ are shown. From Schmidt et al. (2002).

\section{Fission}

Since heavier stable nuclei are more neutron-rich than the lighter ones, the process of fission of a heavy nucleus is a source of neutron-rich medium mass nuclei. In addition, fission is one of the important decay channels of excited heavy nuclei. Thus, it plays a role as a direct source of exotic nuclei and as a process interfering with other reactions used for this purpose. Applications of fission to generate neutron-rich nuclei differ in methods used to excite a fissile nucleus and in the range of excitation energies imparted. On the low-excitation end is the spontaneous fission and the thermal-neutron induced fission (Rochman et al., 2004, Wahl, 1988). To this class belongs also fission resulting from electromagnetic excitation (photofission) (Cetina et al. 2002). The photons inducing fission may be produced directly, e.g. by converting an intense electron beam into bremsstrahlung (Diamond, 1999) or they can be virtual, resulting from a fast motion of a fissile system relative to a high- $Z$ tar- get (Bertulani and Baur, 1986). The higher excitation energies are achieved by bombarding fissile targets with beams of fast neutrons or charged particles. Low energy proton-induced fission is a frequent choice because of the relative technical simplicity. The main aspects of this method are discussed by Penttilä et al. (2010) who recently developed a novel method to measure the particleinduced fission yields. The high energy reactions induced by light or heavy ions lead to high excitation energies and subsequent fission becomes one of the main deexcitation channels influencing the outcome of the spallation and fragmentation reactions, respectively. High energy reactions in inverse kinematics, where a heavy fissile nucleus is the projectile, has been proven to be exceptionally fruitful. The pioneering experiments with relativistic ${ }^{238} \mathrm{U}$ beam at GSI Darmstadt revealed the properties and advantages of this approach (Bernas et al., 1994, 1997). When the target nucleus has a large $Z$ number, the excitation of a fissile projectile-like fragment has a nuclear contribution (fragmentation) and an electromagnetic one 


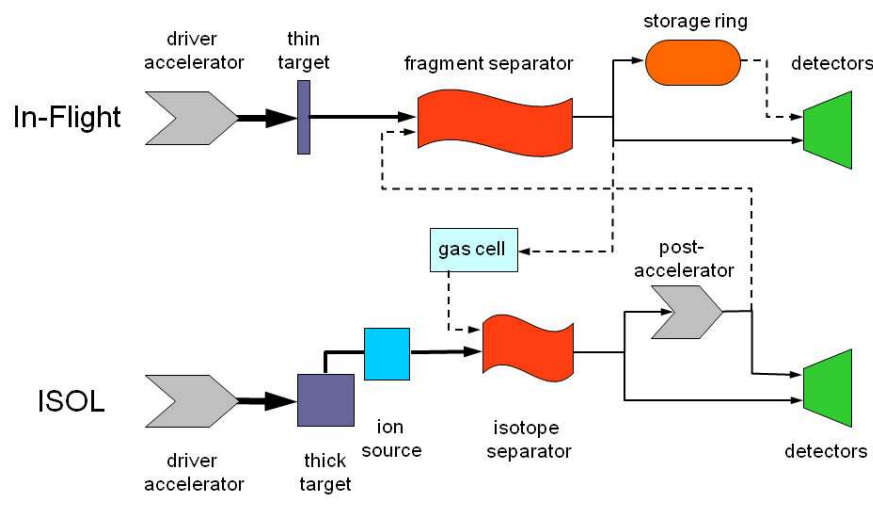

FIG. 8 (Color online) A general scheme of the two main methods used to extract and separate radioactive nuclei. The dashed lines indicate connections which are considered in planning future facilities.

(photofission). This situation was systematically investigated in the reaction of $1 \mathrm{GeV} /$ nucleon ${ }^{238} \mathrm{U}$ impinging on a lead target (Enqvist et al., 1999). A similar technique was applied in a broad campaign dedicated to the comprehensive study of spallation of heavy nuclei. The contribution of fission was investigated in detail for the spallation of ${ }^{238} \mathrm{U}$ by hydrogen (Bernas et al. 2003 ) and by deuterium (Pereira et al., 2007), as well as of ${ }^{208} \mathrm{~Pb}$ by hydrogen (Fernández-Domínguez et al. 2005).

The emerging general picture of the production of nuclei in both the spallation and the fragmentation reactions with inclusion of the fission channel was discussed by Schmidt et al. (2002). It is illustrated in Figure 7 which shows the production cross sections for beams of ${ }^{208} \mathrm{~Pb}$ and ${ }^{238} \mathrm{U}$ impinging at $1 A \mathrm{GeV}$ on hydrogen, deuterium, and lead targets. Although results of model calculations are shown in Figure 7, they represent very well features observed in experiments.

Recently, in the new-generation RIBF facility at RIKEN Nishina Center, the in-flight fission of a 345 $\mathrm{MeV} /$ nucleon ${ }^{238} \mathrm{U}$ beam has been used to produce 45 new neutron-rich isotopes (Ohnishi et al. 2010).

\section{B. Separation}

In reactions used to produce radioactive nuclei always a large number of different products is formed and some method of selection is necessary to filter nuclei of interest from an unwanted background. Very generally, one can classify all separation methods which are used into two distinct classes. The main difference is the target thickness relative to the range of products in the target material. If the relative target thickness is small, the products emerge from the target with significant kinetic energy and can be promptly manipulated by ionoptical devices. The separation techniques of this type are called In-Flight and the filtering devices are called recoil or fragment separators. On the other hand, if the production target is relatively thick, such that the products are stopped in its volume, the nuclei of interest have to be extracted from the target for further filtering. For historical reasons such approach is referred to as ISOL (which stands for Isotope Separator On Line) technique. The principle of operation of In-Flight and ISOL type of facility is shown schematically in Figure 8. In the following we discuss briefly only basic features of both these methods and provide selected examples of laboratories in which they are implemented, referring the reader to technical papers with detailed information. A comparison of the two separation methods with a discussion of some future prospects, including the hybrid combination, was made by Tanihata (2008).

\section{In-Flight}

The key feature of the In-Flight methods is that the kinetic energy of the reaction product is large enough to escape from the relatively thin production target. This method is applicable to reactions induced by heavy ions like fusion-evaporation, multi-nucleon transfer and projectile fragmentation or fission in inverse kinematics. The products emerging from the target enter an ion-optical system of magnetic and electric fields where they are separated from unwanted contaminants and delivered to the final experimental station. Usually, the main selection is applied to the mass-over-charge ratio $A / q$ of particles by means of a uniform magnetic field, according to the formula relating the magnetic field $B$ with the momentum $p$ of a particle having charge $q$ and moving in this field along a circular trajectory of the radius $\rho$ :

$$
B \rho=\frac{p}{q}=u \beta \gamma \frac{A}{q},
$$

where $u$ is the atomic mass unit, $\beta$ is the particle velocity, and $\gamma$ is the Lorentz factor $\left(\gamma=1 / \sqrt{1-\beta^{2}}\right)$. Additionally, in some separators the crossed magnetic and electric fields are used to select the velocity of particles (Wien filter). The In-Flight method is fast as the typical time of flight through the separator is of the order of a microsecond or shorter. Another important feature is the lack of chemical sensitivity.

At the low-energy end, the fusion reaction requires projectiles with the energy of the order of $10 \mathrm{MeV} /$ nucleon (Coulomb barrier). The target thickness is usually of the order of $1 \mathrm{mg} / \mathrm{cm}^{2}$. The average energy of the reaction product results simply from the momentum conservation in a system of collision partners. For this reason, the filtering devices in this case are called recoil separators. The energy of the recoiling products is usually too small to allow for the in-flight identification of ions. The general properties of recoil separators were reviewed by Davids (2003). 
TABLE I The leading laboratories employing the In-Flight method to produce and study the radioactive decays very far from the stability. In the lower part of the table the facilities under construction are listed.

\begin{tabular}{|c|c|c|c|c|c|c|}
\hline Country & Laboratory & $\begin{array}{l}\text { Driver } \\
\text { accelerator }\end{array}$ & Beams & $\begin{array}{c}\text { Max beam } \\
\text { energy }[A \mathrm{MeV}]\end{array}$ & Separator & Reference \\
\hline Finland & Jyväskylä & cyclotron & $\mathrm{Ne}-\mathrm{Kr}$ & $\simeq 10$ & RITU & Leino et al. (1995) \\
\hline Germany & GSI & linac & $\mathrm{H}-\mathrm{U}$ & 11 & SHIP & Münzenberg et al. (1979) \\
\hline USA & ORNL & tandem & $\mathrm{H}-\mathrm{U}$ & $\simeq 10$ & RMS & Gross et al. $(2000)$ \\
\hline USA & ANL & tandem + linac & $\mathrm{H}-\mathrm{U}$ & 17 & FMA & Davids et al. (1992) \\
\hline Russia & FLNR & cyclotron & $\mathrm{Li}-\mathrm{Ar}$ & 50 & ACCULINNA & Rodin et al. (2003) \\
\hline China & HIRFL & cyclotron & $\mathrm{C}-\mathrm{U}$ & 60 & RIBLL & Sun et al. $(2003)$ \\
\hline France & GANIL & 2 cyclotrons & $\mathrm{C}-\mathrm{U}$ & 95 & LISE & Mueller and Anne (1991) \\
\hline USA & NSCL & 2 cyclotrons & $\mathrm{O}-\mathrm{U}$ & 170 & A1900 & Morrissey et al. (2003) \\
\hline Japan & RIBF & 4 cyclotrons & $\mathrm{H}-\mathrm{U}$ & 350 & BigRIPS & Sakurai (2008) \\
\hline Germany & GSI & synchrotron & $\mathrm{H}-\mathrm{U}$ & 1000 & FRS & Geissel et al. (1992) \\
\hline USA & FRIB & linac & $\mathrm{H}-\mathrm{U}$ & 500 & & Thoennessen (2010) \\
\hline Germany & FAIR & synchrotron & $\mathrm{H}-\mathrm{U}$ & 1500 & Super-FRS & Winkler et al. (2008) \\
\hline
\end{tabular}

By increasing the energy and shifting to the projectile fragmentation regime thicker targets can be used. At the energy of about $50 A \cdot \mathrm{MeV}$ the typical targets have a few hundred $\mathrm{mg} / \mathrm{cm}^{2}$ thickness, while at $1000 A \cdot \mathrm{MeV}$ they reach the thickness of a few $\mathrm{g} / \mathrm{cm}^{2}$. At the larger projectile energies the kinematical focusing helps to achieve larger acceptance by the electro-magnetic fragment separator. Additional filtering, according to the atomic number $Z$ of the particle, is achieved by means of an energy degrader mounted in the middle of the fragment separator. The high energy of the reaction products allows for their full in-flight identification by means of time-offlight and energy-loss measurements for individual ions. The resulting extreme sensitivity is one of the most important advantages of the medium- and high-energy InFlight technique. Indeed, this was the key factor allowing the first observation of doubly-magic nuclei ${ }^{100} \mathrm{Sn}(\mathrm{Le}-$ witowicz et al., 1994; Schneider et al., 1994), ${ }^{78} \mathrm{Ni}$ (Engelmann et al., 1995), and ${ }^{48} \mathrm{Ni}$ (Blank et al., 2000), as well as the discovery of the two-proton radioactivity (Giovinazzo et al., 2002, Pfützner et al., 2002).

Although the transmission, separation, and in-flight identification of reaction products are easier at large projectile energy, the maximum beam intensity which can be achieved decreases with increasing energy. Moreover, the quality of the beam of fragmentation products is rather poor. The energy spread of the fragments resulting from both the reaction kinematics and the energy-loss straggling in layers of matter hinders efficient stopping in the final detectors. Hence, the optimal conditions for production of a given nucleus result from a compromise between different factors.

The special role of the high-energy In-Flight method comes from a relatively simple way of delivering beams of radioactive nuclei. Such radioactive beams may be in- jected into more sophisticated devices like storage rings (Nolden et al. 2008) or ion traps (Kluge et al., 2008), or can be used to induce secondary reactions leading to a significant expansion of the nuclear physics field of research.

The leading facilities using the In-Flight method to study decays of very exotic nuclides together with their brief characteristics and the corresponding reference to the detailed information are collected in Table 1 .

\section{ISOL}

In an ISOL-type facility the nuclei of interest are produced in a relatively thick target irradiated by a primary beam from a driver accelerator. If the products recoil from the target they are stopped by means of a catcher or a gas cell, otherwise they diffuse out of the target material. Subsequently they are transferred to the ion source and extracted, mostly as $1^{+}$ions, by means of an $\mathrm{HV}$ potential of the order of $50 \mathrm{kV}$. Because of the constant charge of the extracted ions, the following separation in a uniform magnetic field corresponds to the mass separation, see Eq. 7. In the first realizations of this technique, such mass separated, very low energy ions were deposited on a thin catcher foil in front of a detection system. In the modern variant of this technique the mass separated ions are postaccelerated and a high quality beam is formed allowing better manipulation of the ions and inducing secondary reactions (JPG, 2011).

Although all kinds of nuclear reactions can (and are) employed in this method, the most important are spallation induced by protons, and fission of target nuclei induced by protons, light ions or neutrons. The latter can come either from a reactor or from a beam of deuterons hitting a neutron converter in front of the target. An in- 
TABLE II The main facilities based on the ISOL method for the radioactive decay studies far from the stability. In the lower part of the table the facilities under construction or planning are listed.

\begin{tabular}{|c|c|c|c|c|c|c|}
\hline \multirow[t]{2}{*}{ Country } & \multirow[t]{2}{*}{ Laboratory } & \multirow[t]{2}{*}{ Facility } & \multicolumn{2}{|r|}{ Driver } & \multirow{2}{*}{$\begin{array}{l}\text { Postaccelerator, } \\
\text { energy }[A \mathrm{MeV}]\end{array}$} & \multirow{2}{*}{ Reference } \\
\hline & & & accelerator & beam & & \\
\hline Finland & Jyväskylä & IGISOL & cyclotron & \multirow{2}{*}{$\begin{array}{c}\mathrm{H}-\mathrm{Xe}, 130 q^{2} / A \mathrm{MeV} \\
\mathrm{H}-\mathrm{Ni}, 10 \mathrm{AMeV}\end{array}$} & \multirow{4}{*}{$\begin{array}{c}\text { tandem, } 8 \\
\text { tandem, } \simeq 10\end{array}$} & Äystö (2001) \\
\hline Belgium & Louvain-La-Neuve & LISOL & cyclotron & & & Kudryavtsev et al. (2008), \\
\hline Italy & INFN-LNS & EXCYT & cyclotron & \multirow{2}{*}{$\begin{array}{c}A<48,80 \mathrm{AMeV} \\
p, d, \alpha, 42-85 \mathrm{MeV}\end{array}$} & & Cuttone et al. (2008) \\
\hline USA & ORNL & HRIBF & cyclotron & & & Stracener (2003) \\
\hline France & GANIL & SPIRAL & 2 cyclotrons & $\mathrm{H}-\mathrm{U}, 95 \mathrm{AMeV}$ & \multirow{2}{*}{$\begin{array}{l}\text { cyclotron, } 25 \\
\text { linac, } 11\end{array}$} & Villari (2003) \\
\hline Canada & TRIUMF & ISAC & cyclotron & $p, 500 \mathrm{MeV}$ & & \multirow{2}{*}{\begin{tabular}{l|} 
Shotter \\
Voulot et al.
\end{tabular}} \\
\hline Switzerland & CERN & REX-ISOLDE & synchrotron & $p, 1.4 \mathrm{GeV}$ & linac, 3 & \\
\hline Italy & LNL & SPES & cyclotron & \multirow{4}{*}{$\begin{array}{c}d, 40 \mathrm{MeV} ; \mathrm{HI}, 14.5 \mathrm{~A} \mathrm{MeV} \\
e, 50 \mathrm{MeV} \\
p, 1 \mathrm{GeV}\end{array}$} & \multirow{4}{*}{$\begin{array}{c}\text { linac, } 11 \\
\text { cyclotron, } 25 \\
\text { linac, } 11 \\
\text { linac, } 150\end{array}$} & \begin{tabular}{|l|l|} 
Cinausero et al. & (2009)
\end{tabular} \\
\hline France & GANIL & SPIRAL-2 & linac & & & Lewitowicz (2008) \\
\hline Canada & TRIUMF & ARIEL & e-linac & & & http://www.triumf.ca/ariel \\
\hline \multicolumn{2}{|c|}{ to be decided } & EURISOL & linac & & & Blumenfeld et al. (2009) \\
\hline
\end{tabular}

teresting concept is to induce fission by bremsstrahlung photons originating from a very high intensity electron beam hitting the high- $Z$ converter (Cheikh Mhamed et al. 2008). The main point is that the delivered intensities of light beams, like protons, deuterons, or electrons, can be significantly larger than maximal intensities of heavy ion beams. This, in combination with thick targets which can be used, results in the high yields of radioactive nuclei which is the main advantage of the ISOL method. On the other hand, the transfer processes occurring in the target and in the ion source take time of the order of milliseconds (see Figure 2) which imposes limits on the half-lives which can be accessed by this method. In addition, some of these processes exhibit chemical sensitivity which for example hinders extraction of refractory elements in some implementations of this technique.

In the last decades, a remarkable progress in ion-source techniques and in manipulating low-energy ions has been achieved (Lecesne, 2008; Wenander, 2008). A spectacular example is the application of resonant laser ionization allowing extremely efficient and clean extraction of selected elements from the source (Cheal and Flanagan, 2010).

A selection of facilities employing the ISOL method with short characteristics and the reference to the corresponding technical information is presented in Table II.

\section{Future facilities}

In the lower part of both Table II and Table II the future facilities which are being constructed or planned are listed. A new idea which is considered in these recent developments is to combine advantages of both the In-Flight and the ISOL methods into hybrid solutions (Tanihata, 2008). Sufficient postacceleration of the ISOL secondary beam may enable taking advantage of instrumentation developed for the In-Flight technique. Such an option is discussed in the EURISOL design study
(Blumenfeld et al. 2009). On the other hand, the fast In-Flight fragment beam may be stopped in a gas cell and extracted at low energy with help of the ISOL techniques (Facina et al. , 2008). An example of such a solution will be realized in the currently constructed FRIB facility (Thoennessen, 2010). The possible connections between the two main approaches to extract and separate radioactive nuclei are marked in Figure 8 by dashed lines.

\section{Detection}

The detection of a radioactive decay requires detection of particle(s) emitted in the process. The large majority of nuclides decay by $\beta$ transitions where primarily an electron or a positron is emitted (the presence of neutrinos can be safely neglected in this context) and in the second step electromagnetic radiation follows in form of $\gamma$ radiation if the daughter nucleus was formed in an excited state and/or of characteristic $X$-rays if the final atom was excited. This secondary electromagnetic deexcitations may proceed by emission of Auger electrons. When we move away from the stability line, however, the mass differences between isobars (and thus the beta decay energy windows) increase and the particle unbound states become populated. On the neutron-deficient side this leads to the $\beta$-delayed charged particle emission, mainly of protons. Beyond the proton-drip line the direct emission of protons comes into play. That is why the detection of particles like $p$ and $\alpha$ plays the central role in the radioactivity studies at the neutron-deficient limit of stability. In turn, on the neutron-rich side emission of $\beta$-delayed neutrons becomes important which makes the neutron spectroscopy necessary. However, also charged particles, like $d, t$, and $\alpha$, are emitted following $\beta$ decay of very neutron-rich nuclei. In the following we sketch the modern methods used to detect charged particles and 
Some abbreviations used in Table I and II

ANL Argonne National Laboratory, USA

FAIR Facility for Antiproton and Ion Research, Darmstadt, Germany

FLNR Flerov Laboratory of Nuclear Reactions at Joint Insitute for Nuclear Research, Dubna, Russia

FRIB Facility for Rare Isotope Beams at Michigan State University, East Lansing, USA

GANIL Grand Accélérateur National d'Ions Lourds, Caen, France

GSI Helmholtzzentrum für Schwerionenforschung GmbH, Darmstadt, Germany

HRIBF Holifield Radioactive Ion Beam Facility at ORNL, USA

HRIFL Heavy Ion Research Facility in Lanzhou, China

INFN-LNS Istituto Nazionale di Fisica Nucleare, Laboratori Nazionali del Sud, Catania, Italy

LNL Laboratori Nazionali di Legnaro, Legnaro, Italy

NSCL National Superconducting Cyclotron Laboratory by Michigan State University, East Lansing, USA

ORNL Oak Ridge National Laboratory, Oak Ridge, USA

RIBF Radioactive Isotope Beam Factory at RIKEN laboratory, Wako, Saitama, Japan

BigRIPS Big RIKEN Projectile Fragment Separator at RIBF

EXCYT EXotics with CYclotron and Tandem at INFNLNS

FMA Fragment Mass Analyser at ANL

FRS FRagment Separator at GSI Darmstadt

IGISOL Ion Guide and Isotope Separator On-Line

LISE Ligne d'Ions Super Epluchés at GANIL

LISOL Leuven Isotope Separator On-Line

RIBLL Radioactive Ion Beam Line in Lanzhou at HRIFL

RITU Recoil Ion Transport Unit

RMS Recoil Mass Spectrometer at HRIBF

SHIP Separator for Heavy Ion reaction Products at GSI Darmstadt

SPES Selective Production of Exotic Species at LNL

SPIRAL Systèeme de Production d'Ions Radioactifs avec Accélération en Ligne at GANIL

neutrons. Finally, we discuss briefly the digital signal processing techniques which represent a new development in the systems of nuclear data acquisition.

The technique of $\gamma$ spectroscopy is the large subject in its own, as it is the main source of detailed data on the nuclear excited states. Here we refrain from discussing its development referring the reader to the rich literature on this topic (Farnea et al. 2010 ; Gelletly and Eberth, 2006, Lee et al. 2003).

\section{Charged particles}

The most common devices for detecting charge particles are based on silicon detectors which record the energy deposit of a charge particle passing through its material. To increase the sensitivity of the measurement and to provide an additional information about the particle, stacks of two or more detectors are frequently used. In such a telescope the energy loss information from thin transmission detectors is combined with the total kinetic energy from the final thick detector. A particle telescope may combine a thin gas chamber, acting as a transmission counter with a thick silicon detector (Axelsson et al. 1998; Moltz et al. 1994). Additional information on the location of the particle's hit can be extracted from a position sensitive detectors where the signal is read from two ends of a resistive electrode. A significant advance in detection technique was introduced with a concept of a silicon strip detector. Particularly successful was the development of a double sided silicon strip detector (DSSSD) with perpendicular sets of strip electrodes on its both sides (Sellin et al., 1992). The achieved granularity provides not only a simple measure of the position but allows to establish a position correlations between subsequent events, like the implantation of an ion and its decay by particle emission in condition of the high total counting rate. In addition, it reduces the effect of energy summing between $\beta$-delayed particles and electrons which deposit much less energy in a strip (a pixel) area (Büscher et al. 2008). Most of results on $\beta$-delayed proton emission (Sec. IV) and on proton radioactivity (Sec. V) were obtained with help of such detectors. An example of a recent improvement is a novel design of a large-area DSSSD with an ultra-thin dead layer (Tengblad et al., 2004). The modern detection set-ups which require granularity but also a large angular coverage are usually constructed as arrays of silicon detectors. They may consist of a large number of simple Si diodes (Fraile and Äystö, 2003), a box of DSSSD detector (Adimi et al. 2010), or a combination of DSSSD detectors with a gaseous multiwire proportional chamber and germanium detectors (Page et al. 2003). In the decay studies at projectile fragment separators (the In-Flight method) the large area of the final focal plane and the large range distributions of selected ions have to be taken into account. To meet the latter challenge, the stacks of many DSSSD detectors are used. The example solution used for the $\beta$-decay studies of ${ }^{100} \mathrm{Sn}$ at the FRS separator (GSI Darmstadt) consisted of three large-area DSSSD detectors, providing in total $3 \times 60 \times 40=7200$ pixels, sandwiched between two sets of ten single-side silicon strip detector (Eppinger et al. 2009). In another development for the FRS separator, a set of two rows consisting of three $5 \mathrm{~cm} \times 5 \mathrm{~cm}$ DSSSD detectors, each with $256(16 \times 16$ strips $)$ pixels was developed (Kumar et al. 2009). Even more ambitious Advanced Implantation Detector Array (AIDA), to be used 
at the FAIR facility, will comprise twenty four $8 \times 8 \mathrm{~cm}^{2}$ DSSSD detectors with $128 \times 128$ strips (Davinson, 2010).

The implantation of a nucleus which undergoes a multiparticle decay into a silicon detector has a serious limitation that only the total decay energy can be measured. The energy sharing between products and their momentum correlations cannot be accessed. Such problem appeared in case of the two-proton radioactivity. The first observation of this decay mode was accomplished by implanting ions of ${ }^{45} \mathrm{Fe}$ into a stack of silicon detectors (Pfützner et al., 2002) and into a DSSSD detector (Giovinazzo et al., 2002). The information on the decay time and on the total decay energy were sufficient to claim the new type of radioactivity, but for the detailed study of this process a novel experimental approach was necessary. To meet this challenge, two new developments were undertaken, both based on a principle of the time projection chamber (TPC). The key idea is that such a gaseous ionization chamber can record tracks of charged particles, allowing their reconstruction in three dimensions. The radioactive ion stopped inside the active volume and the subsequently emitted particles ionize the counting gas. The primary ionization electrons drift in a uniform electric field towards the charge amplification section producing the two-dimensional representation of the particles' tracks. The drift time contains the position information along the electric field direction. In one solution, the amplified ionization charges are collected electronically by means of an anode plate with two sets of orthogonal strips (Blank et al. 2010). This detector rendered the first direct evidence for the two protons emitted in the decay of ${ }^{45} \mathrm{Fe}$ (Giovinazzo et al. 2007 ). In the second design, the idea of an optical readout (Charpak et al. 1988) was implemented. It is based on the observation that light is emitted in the final stage of charge amplification. In the Optical Time Projection Chamber (OTPC) this light is collected by a CCD camera and by a photomultiplier (PMT) (Miernik et al., 2007a). The construction of this detector is shown schematically in Figure 9. The application of the OTPC yielded spectacular results, including the detailed proton-proton correlation picture for the $2 p$ decay of ${ }^{45} \mathrm{Fe}$ (Miernik et al. 2007c) and the first observation of the $\beta$-delayed three-proton emission channel (Miernik et al., 2007b). An example event of the two-proton radioactivity of ${ }^{45} \mathrm{Fe}$ is shown in Figure 10.

In case of very short decay half-lives, in the subnanosecond range, the implantation technique generally cannot be used. A short-lived precursor decays in-flight very close to the place of its production. The identification of the nucleus and its properties can be deduced from the detection and tracking of all decay products. This approach was successfully applied to the study the $2 p$ decay of ${ }^{19} \mathrm{Mg}$ (Mukha et al., 2007) which also exemplifies advantages of radioactive beams. Ions of ${ }^{19} \mathrm{Mg}$ were produced in a secondary target by a neutron knock-out reaction from a beam of ${ }^{20} \mathrm{Mg}$ delivered by the GSI FRS

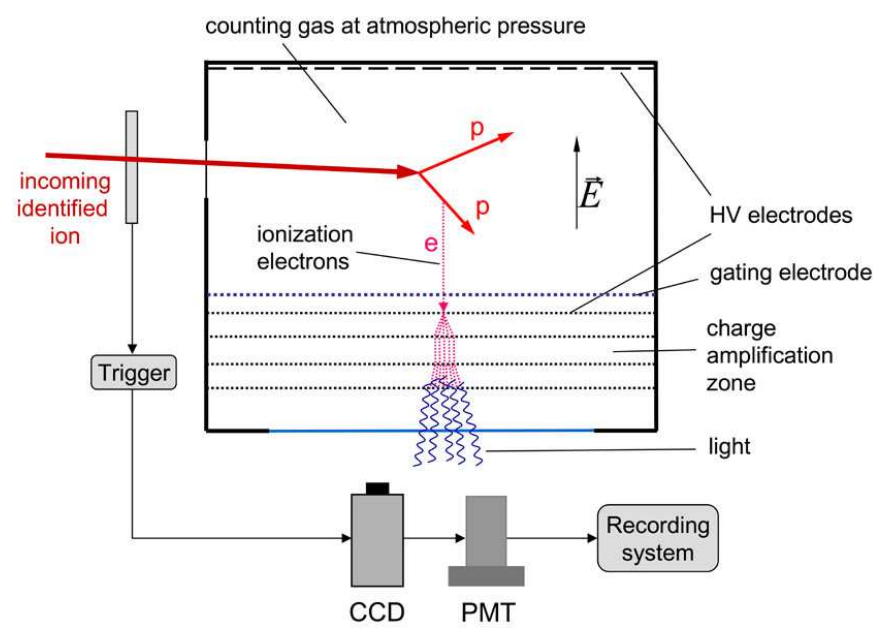

FIG. 9 (Color online) A schematic view of the Optical Time Projection Chamber (OTPC). For each recorded event, the data consist of a 2D image taken by a CCD camera in a given exposure time and the total light intensity detected by a photomultiplier (PMT) as a function of time, sampled by a digital oscilloscope. The gating electrode is used to block the charge induced by incoming ions.

separator (Geissel et al., 1992). The tracking of emitted protons by means of silicon microstrip detectors (Stanoiu et al. 2008) allowed to establish the longitudinal distribution of decay vertexes and to determine the half-life of ${ }^{19} \mathrm{Mg}$ to be $4.0(15) \mathrm{ps}$. At the same time the information on correlations between emitted protons was collected. Since the beam impinging on the secondary target contains usually a mixture of different ions ("cocktail" beam) other reactions can be addressed simultaneously. For example, in the measurement of ${ }^{19} \mathrm{Mg}$, the data on proton and two-proton decays from ${ }^{15} \mathrm{~F},{ }^{16} \mathrm{Ne}$, and ${ }^{19} \mathrm{Na}$ were obtained (Mukha et al. 2010). Similar technique has been applied to study two-proton emission form excited states of ${ }^{17} \mathrm{Ne}$ (Chromik et al., 2002, Zerguerras et al. 2004). The tracking method of the in-flight decay products is expected to provide information on several $2 p$ emitters among light nuclei, see Sec. VII

\section{Neutrons}

Currently, two different methods for neutron detection are used in nuclear spectroscopy. The first one is based on thermal-neutron induced reactions, like ${ }^{3} \mathrm{He}(\mathrm{n}, \mathrm{p})^{3} \mathrm{H}$, ${ }^{6} \mathrm{Li}(\mathrm{n}, \alpha)^{3} \mathrm{H}$, or ${ }^{10} \mathrm{~B}(\mathrm{n}, \alpha)^{7} \mathrm{Li}$, leading to charged particles which can be easily detected. The neutrons emitted by a radioactive source have to be first thermalized and this is achieved by means of a moderator - usually a large block of polyethylene surrounding the source. In the moderator cylindrical cavities, arranged in concentric rings, are drilled in which proportional counters are mounted (Mehren et al., 1996). In these counters which 

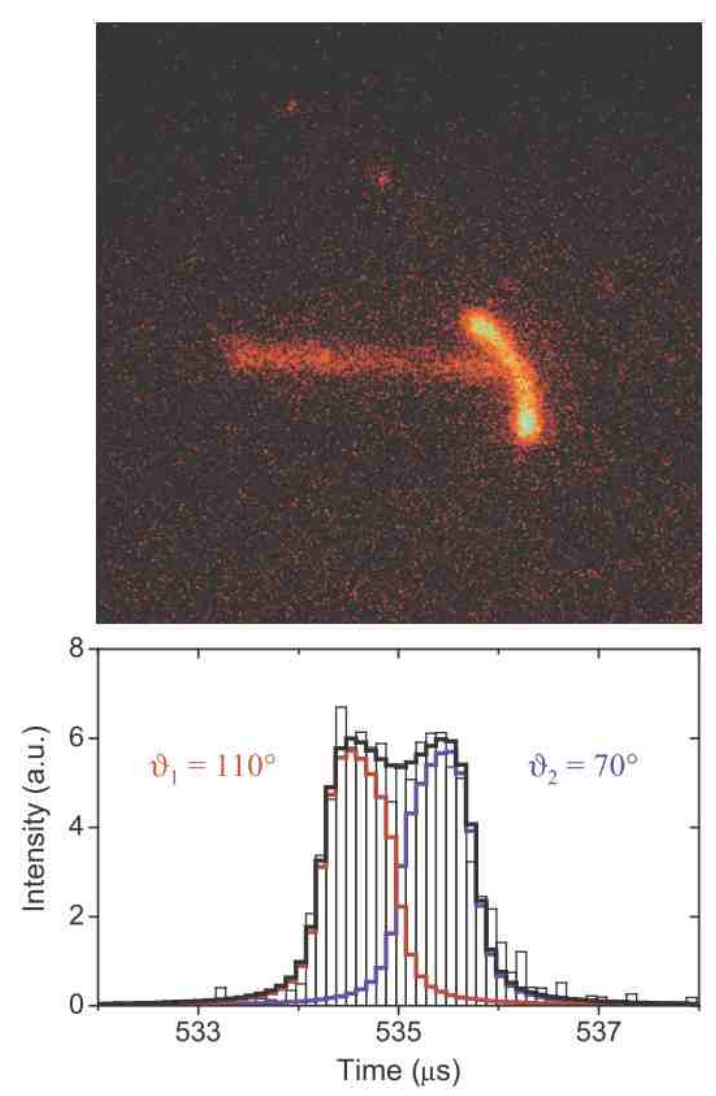

FIG. 10 (Color online) An example of a registered two-proton decay event of ${ }^{45} \mathrm{Fe}$. Top: an image recorded by the CCD camera in a $25 \mathrm{~ms}$ exposure. A track of a ${ }^{45} \mathrm{Fe}$ ion entering the chamber from left is seen. The two bright, short tracks are protons of approximately $0.6 \mathrm{MeV}$, emitted $535 \mu$ s after the implantation. Bottom: a part of the time profile of the total light intensity measured by the PMT (histogram) showing in detail the $2 \mathrm{p}$ emission. Lines show results of the reconstruction procedure yielding the emission angles $\vartheta$ with respect to the axis normal to the image. From Miernik et al. (2007c).

are filled with ${ }^{3} \mathrm{He}$ or $\mathrm{BF}_{3}$ gas, neutron-capture reactions take place and are detected. Such construction allows to cover a large solid angle, approaching $4 \pi$ and the large total efficiency of up to $30 \%$ can be achieved for a broad neutron energy-range from meV to tens of $\mathrm{MeV}$ and almost independent on the neutron energy due to thermalization. Since the information on energy is lost, such detector is used primarily for counting which makes it well suited for determination of branching ratios for various neutron emission channels. Another disadvantage of the moderation is that a neutron is detected up to about $100 \mu$ s after the emission. Such a delay reduces the total counting rate which can be accepted. An example of a modern version of such a $4 \pi$ neutron counter is the NERO detector, recently built at the NSCL laboratory (Pereira et al. 2010). Its layout is shown in Figure 11.

A different, and to a large degree a complementary solution, employs scintillation detectors in which inter-
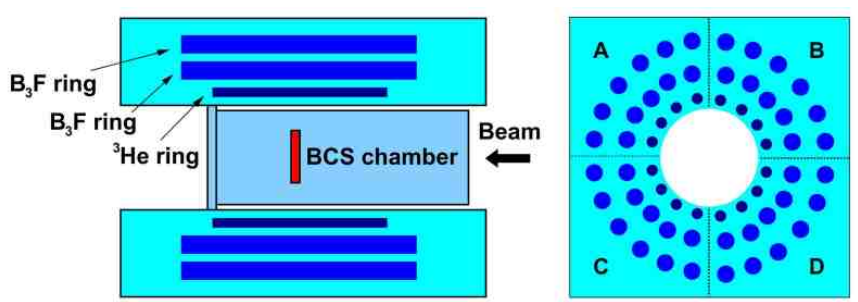

FIG. 11 (Color online) Schematic drawings of the NERO detector. Left: side view showing the Beta Counting Station (BCS) chamber located inside of NERO with the DSSSD at the central position. Right: backside showing the cylindrical cavity to house the BCS and the three concentric rings of gas-filled proportional counters. The labels A, B, C and D designate the four quadrants. From Pereira et al. (2010).

actions of fast neutrons are detected, predominantly by recording elastic proton recoils. The neutron energy can be then determined by means of the time-of-flight (TOF) after a trigger signal, given for example by a $\beta$ particle. The panels containing liquid or plastic scintillators are mounted at some distance from the radioactive source, which usually reduces the solid angle which can be achieved. The efficiency depends on the neutron energy and exhibits a low-energy threshold at about few hundred $\mathrm{keV}$. In addition, such detector is sensitive also to $\gamma$ radiation and the pulse-shape analysis has to be performed for the $n-\gamma$ discrimination (Skeppstedt et al., 1999). Such an approach to the neutron TOF spectroscopy can be exemplified by the detector TONNERRE developed at the GANIL laboratory (Buta et al. 2000).

\section{Signal processing}

In the conventional approach, signals from detectors of nuclear radiation are preamplified and then processed in analogue-electronics modules like shapers, amplifiers, discriminators, etc., to be finally converted to the digital form in analogue-to-digital (ADC) and time-to-digital (TDC) converters, and stored in the electronic memory of the data acquisition system. With the increasing number of channels which have to be read, resulting from pixelization of the detectors (strips, segments, pads) the amount of necessary electronic units is growing and thus magnifying the complexity and the cost of the instrumentation. In addition, by storing only the values of energy and time for an event, the information on the pulse shape is lost, which is very disadvantageous in some applications.

A possible solution to these problems is offered by the technique of the digital signal processing (DSP) which since recently is taking over the conventional data acquisition systems in nuclear spectroscopy. Its basic principle is that the output of a preamplifier is digitized first and all further manipulations are performed by numerical algorithms acting on this digital representation of 


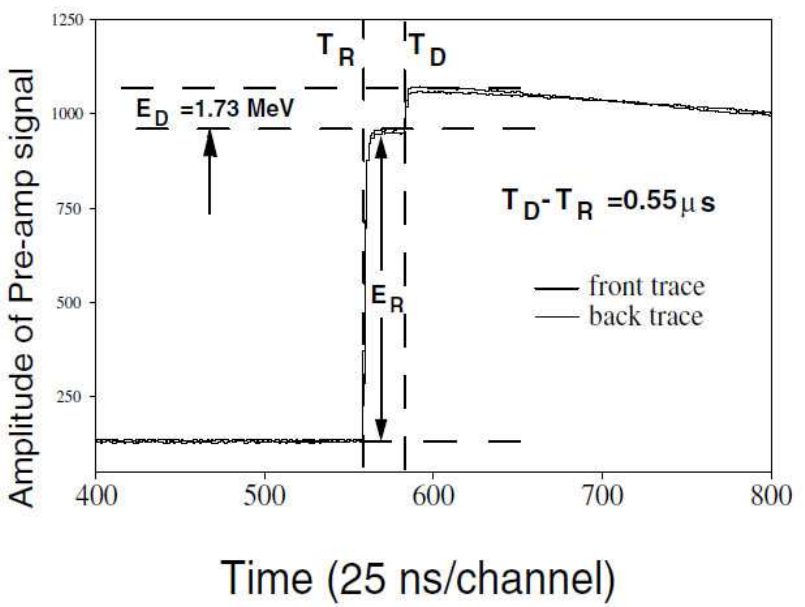

FIG. 12 Part of the preamplifier signal waveforms recorded by the front and the back strip of the $65-\mu \mathrm{m}$ DSSSD during the ${ }^{145} \mathrm{Tm}$ experiment. The recoil depositing about $14 \mathrm{MeV}$ energy is followed after $0.55 \mu$ s by the $1.73 \mathrm{MeV}$ signal. From Karny et al. (2003).

the signal. These algorithms replace all functions of analogue electronics and additionally offer a choice of much more complex and flexible operations on the pulse. Originally, the introduction of the DSP methods was motivated by needs of segmented X-ray and $\gamma$-ray arrays and this sector is the main recipient of this technology (Crespi et al., 2009; Cromaz et al., 2008, Pietri et al., 2007; Starosta et al. , 2009). One commercial development - the Digital Gamma Finder module (DGF-4C) by XIA LLC (Hubbard-Nelson et al., 1999$)$ — proved to be particularly successful also in the domain of particle spectroscopy. Some applications of the DGF electronics in various decay studies of exotic nuclei were reviewed by Grzywacz (2003). The trends and new products from this developer were presented by Warburton and Grudberg (2006).

A good illustration of new possibilities provided by the DSP is the measurement of very short-lived proton radioactivity in ${ }^{145} \mathrm{Tm}$ (Karny et al. 2003 ). The technical challenge is to detect a low-energy proton $(\sim 1.5 \mathrm{MeV})$ emitted very shortly $(\sim 1 \mu \mathrm{s})$ after stopping of the parent nucleus which deposits up to about $35 \mathrm{MeV}$ in the implantation DSSSD detector. Such sequence of events cannot be resolved easily when signals are passed through the analogue amplifiers. The solution offered by the DGF electronics is to store the whole waveforms of the signals from the silicon strips and to analyze their shapes off-line. In addition, the special triggering mode was implemented to the DGF board which allowed to store only those events in which the pile-up of two pulses was detected. This feature leads to the large increase of sensitivity, which is especially important when many different ions are coming to the final detector and the decay investigated is rare. This technique was the key factor leading to the discovery of the fine structure in the decay of ${ }^{145} \mathrm{Tm}$ (Sec. V). An example of the recorded waveform representing a low-energy proton signal superimposed on the large implantation signal of the ${ }^{145} \mathrm{Tm}$ ion is shown in Figure 12. The same method was instrumental in the observation of the superallowed $\alpha$-decay chain from ${ }^{109} \mathrm{Xe}$ (Darby et al. 2010) which is discussed in Sec. VI.

Another development which is recently gaining importance, especially in the domain of detectors with high granularity, is the technology of so called applicationspecific integrated circuit (ASIC). It is based on a highly integrated circuit which is customized for a specific use rather than for a general-purpose application. Usually, one integrated circuit (IC) chip features several independent channels, each capable of handling energy and timing of a single detector element (pixel or strip). In fact, the large silicon array AIDA (Davinson, 2010) as well as one of the TPC detectors developed to study $2 p$ radioactivity (Blank et al., 2010), see Sec. III.C.1, employ ASIC-type chips in their read-out electronic system. An example of multi-channel IC for the detection of nuclear radiation is described by Engel et al. (2011). In combination with an array of Si-strip detectors (Wallace et al. 2007 ) it was used in a study of ${ }^{6} \mathrm{Be}$ (Grigorenko et al. 2009a), presented in Sec. VII.B.1. 


\section{BETA DELAYED PARTICLE EMISSION}

\section{A. Beta decay, general observations}

\section{The beta strength}

The weak interactions and in particular their lowenergy manifestation in nuclear beta decay are by now well understood (Behrens and Büring, 1982; Grotz and Klapdor, 1990), see (Severijns et al., 2006) for a recent survey of weak interaction tests in nuclear physics. The decay rate for allowed $\beta^{-}$or $\beta^{+}$decays can be transformed to give the known expression for the $f t$-value

$$
f t=\frac{K}{g_{V}^{2} B_{F}+g_{A}^{2} B_{G T}},
$$

where $t$ is the partial halflife of the transition, $K / g_{V}^{2}=$ $6144.2(1.6) \mathrm{s}$ and $g_{A} / g_{V}=-1.2694(28)$ (Towner and Hardy, 2010), and $B_{F}$ and $B_{G T}$ are the reduced matrix elements squared for the Fermi and Gamow-Teller parts. (Note that some authors define $B_{G T}$ to include the factor $\left(g_{A} / g_{V}\right)^{2}$.) Nuclear electron capture will also contribute, but is mainly noticeable for low decay energies and in heavier elements. The phase space factor can be approximated roughly by $f=\left(1+Q / m_{e} c^{2}\right)^{5} / 30$ in terms of the decay energy ( $Q$-value) for $\beta^{ \pm}$-decays, more accurate determinations can be found through (Behrens and Büring, 1982 Wilkinson, 1995). Due to the parabolic mass surface the $Q$-values increase as one moves away from betastability which by itself enhances beta decay rates. Empirically the beta halflives fall off approximately exponentially away from stability (Zhang et al., 2007).

There are only few beta decays where most of the beta strength is energetically accessible in the decay, and the detailed distribution in general plays a crucial role. The Fermi strength is concentrated around the Isobaric Analogue State (IAS). The summed strength fulfils a sum rule $\sum B_{F}^{+}-\sum B_{F}^{-}=Z-N$ that involves Fermi transitions in "both directions", this is relevant e.g. for the odd-odd $N=Z$ nuclei from ${ }^{34} \mathrm{Cl}$ to ${ }^{98} \mathrm{In}$ where most ground states have $T=1$ and can be fed as well as decay by Fermi transitions.

The Gamow-Teller strength obeys the Ikeda sum rule:

$$
\sum B_{G T}^{-}-\sum B_{G T}^{+}=3(N-Z),
$$

and much of this strength is collected in the several $\mathrm{MeV}$ wide so-called Gamow-Teller Giant Resonance (GTGR), although mixing with higher lying configurations is important and removes a sizeable part of the strength to higher energies (Ichimura et al. 2006); this is often referred to as the quenching of the GT strength, the key point being that the quenching factor, although depending on what approximations are used when calculating the GT strength, is varying slowly as a function of $N$ and $Z$. Note that the summed value of $B_{G T}$, even including quenching, is larger than $B_{F}$. More details on the strength distribution will be given in the following two subsections.

For the lightest nuclei the $Q$-values are for a given mass number slightly higher on the proton-rich side than on the neutron-rich side of beta-stability and experimentally halflives are systematically shorter on the protonrich side. The asymmetry is enhanced by the contribution from the IAS transition in nuclei with $N<Z$. For masses above 100 the situation has changed: experimentally the halflives for nuclei more than 3-4 nucleons away from the beta-stability line are systematically shorter on the neutron-rich side than on the proton-rich side. For these nuclei the $\beta^{+}$decay increases isospin and the systematic difference can be understood from eq. (9) since the summed $\beta^{-}$strength is significantly higher than the $\beta^{+}$strength.

Several approaches have been employed to reproduce and predict the beta decay halflives in large parts of the nuclear chart (Borzov and Goriely, 2000, Homma et al. 1996; Möller et al. . 2003. Nakata et al., 1997). The increase in computing power has allowed the use of increasingly sophisticated microscopic models (Borzov et al. 2008, Borzov, 2006, Brown, 2001), even ab initio methods (Navrátil et al. , 2007; Pervin et al., 2007) for the very light nuclei, and more extended RPA (Toivanen et al. , 2010) and shell-model calculations are underway (G. Martínez-Pinedo, private communication, 2010).

\section{Delayed particles}

Defining as usual the relative probability $P_{S}$ for a given beta-delayed decay mode $S$ (1p, 2p, 1n, 2n, $\alpha$ etc), as the fraction of all decays that results in a final state containing $S$, one can find the average number of emitted neutrons as $P_{n}=\sum_{i} i P_{i n}$ with $P_{p}$ defined in a similar way. The energetics for the different channels is sketched in figure 13 . The figure implicitly assumes that decays take place through states in the emitter and that multiparticle emission happen sequentially; as will be argued below these assumptions may not hold for all cases. The $Q$-value for delayed emission of neutrons explicitly depends on the neutron separation energies, but also the $Q$-value for some other modes can be rewritten (Jonson and Riisager, 2001) to show that they depend on the neutron separation energies of the precursor nucleus:

$$
Q_{\beta d}=3.007 \mathrm{MeV}-S_{2 n}, \quad Q_{\beta t}=9.264 \mathrm{MeV}-S_{3 n}
$$

Most beta-delayed decay modes will therefore be enhanced at the driplines since multi-nucleon separation energies will be low there: the "dripline" for emission of two or more neutrons will lie very close to the one neutron dripline. We shall return in section IV.D in more detail to the particle emission processes, but can note already now that the probability for a delayed multi-particle emission 


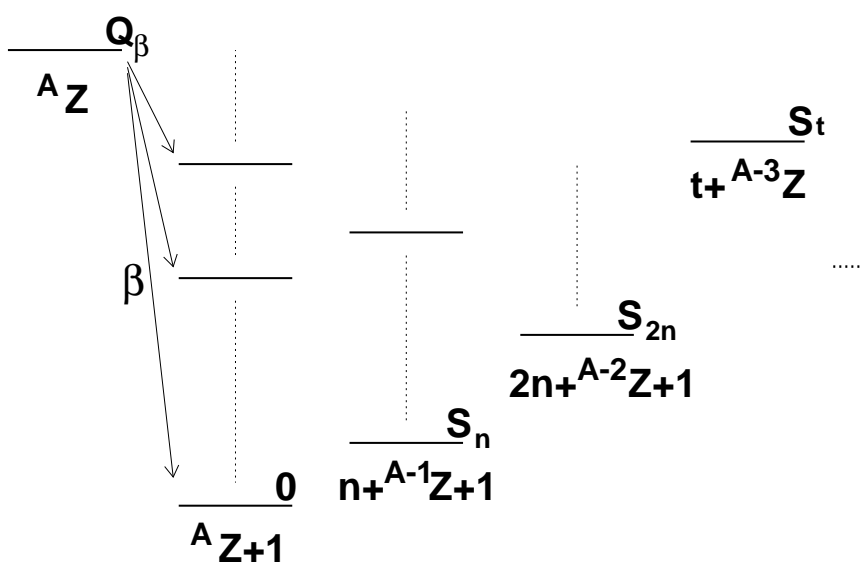

FIG. 13 Some of the possible energetically allowed betadecay channels for a neutron rich nucleus. The precursor, ${ }^{A} Z$, beta decays to the emitter, ${ }^{A}(Z+1)$, that has particle unbound excited states. All energies are given relative to the ground state of the emitter.

may depend on the emission mechanism (simultaneous or sequential emission) as well as on the energy available.

As we approach the driplines, the enhanced role played by beta-delayed particle emission implies that the physics problems investigated via beta decay will overlap more and more with the ones investigated via reaction studies, but the selection rules for beta decay will provide a spin selectivity that often is useful. We shall focus here on the general features of the beta decay processes and specific challenges met in decay studies, but shall give as well selected examples of structure questions that have been studied.

A specific example of this is the population in beta decay of excited states that enter in the astrophysical $r p$-process (Wormer et al., 1994). An even more direct need for beta-decay data in astrophysics arises in processes where weak interactions play a role, either directly as beta decay rates or indirectly where neutrino interactions are important, see (Arnould et al. 2007; Borzov, 2006. Grawe et al., 2007, Langanke and Martínez-Pinedo, 2003).

Quite apart from the general interest in the coupling to continuum degrees of freedom, cf. section I.C.3, beta decay processes may provide specific information on isospin mixing that is expected to be enhanced for continuum states at low energy, see e.g. (Garrido et al., 2007, Michel et al. 2010b, Mitchell et al. 2010).

The experimental considerations were covered in general in section III] but a few specific comments may be relevant. Since beta halflives are longer than about one ms, essentially all experiments make use of a stopped beam. This gives a source distribution that at in-flight facilities may have a considerable spatial extent. Experiments in storage rings (Litvinov and Bosch, 2011) or ion and atom traps (Severijns et al., 2006) have been undertaken in sev- eral cases, but still present practical problems in particular concerning efficient detection of all decay products. Complementary experiments at different type of facilities may overcome the disadvantages for a specific production method, one example being the study of ${ }^{32} \mathrm{Ar}$ (Bhattacharya et al. 2008) where a high-resolution spectrum obtained at ISOLDE/CERN was combined with an absolute intensity determination carried out at NSCL/MSU. ISOL facilities often have problems for very short halflives and in determining absolute activities, whereas in-flight facilities frequently employ composite beams (so-called cocktail beams) where special procedures may be needed in order to correct for background from decay of nonrelevant isotopes, see e.g. (Dossat et al., 2007, KurtukianNieto et al. 2008). As will be seen below many results from the past decade come from in-flight facilities, often through implantation of the radioactive ion into a $\mathrm{Si}$ detector.

Beta-delayed particle emission has been the subject of several earlier reviews, both more general ones (Jonson and Nyman, 1996, Jonson and Riisager, 2001) and specific ones for proton-rich nuclei (Blank and Borge, 2008; Hardy and Hagberg, 1988), neutron-rich nuclei (Hansen and Jonson, 1988) and heavy nuclei (Hall and Hoffman, 1992). Since more detailed accounts can be found there the treatment here, in sections IV.B, IV.C and IV.E will be somewhat brief. The remaining section IV.D deals with beta-delayed multi-particle emission and naturally has more interconnections to other parts of the present paper.

\section{B. $\beta^{+}$delayed emission of one particle}

\section{Occurrence of particle emission}

Figure 14 shows $Q_{E C}$ and the beta-decay halflives for the most proton-rich nuclei where beta-decay still plays a role. There is considerable scatter in the values, but also clear effects of the proton shells at $Z=50$, enhanced by the fact that Fermi transitions contribute below this value and not above, and $Z=82$ as well as the neutron shell at 82 corresponding to $Z=72$ : below this the competing decay mode is proton emission, above alpha decay takes over inside the proton dripline. For beta decays along the proton dripline (dashed lines in the figure) one still finds that the $Q$-values decrease towards $10 \mathrm{MeV}$ in the heavier nuclei. The scatter indicates that local nuclear structure still plays an important role in these decays. Even though protons and neutrons in many cases still are within the same major shell, forbidden decays will play an important role for the heavier nuclei.

The Coulomb barrier plays a dominating role in betadelayed particle emission in proton-rich nuclei, as illustrated in figure 15 , and essentially limits the emitted particles to protons. Delayed alpha emission is energetically 

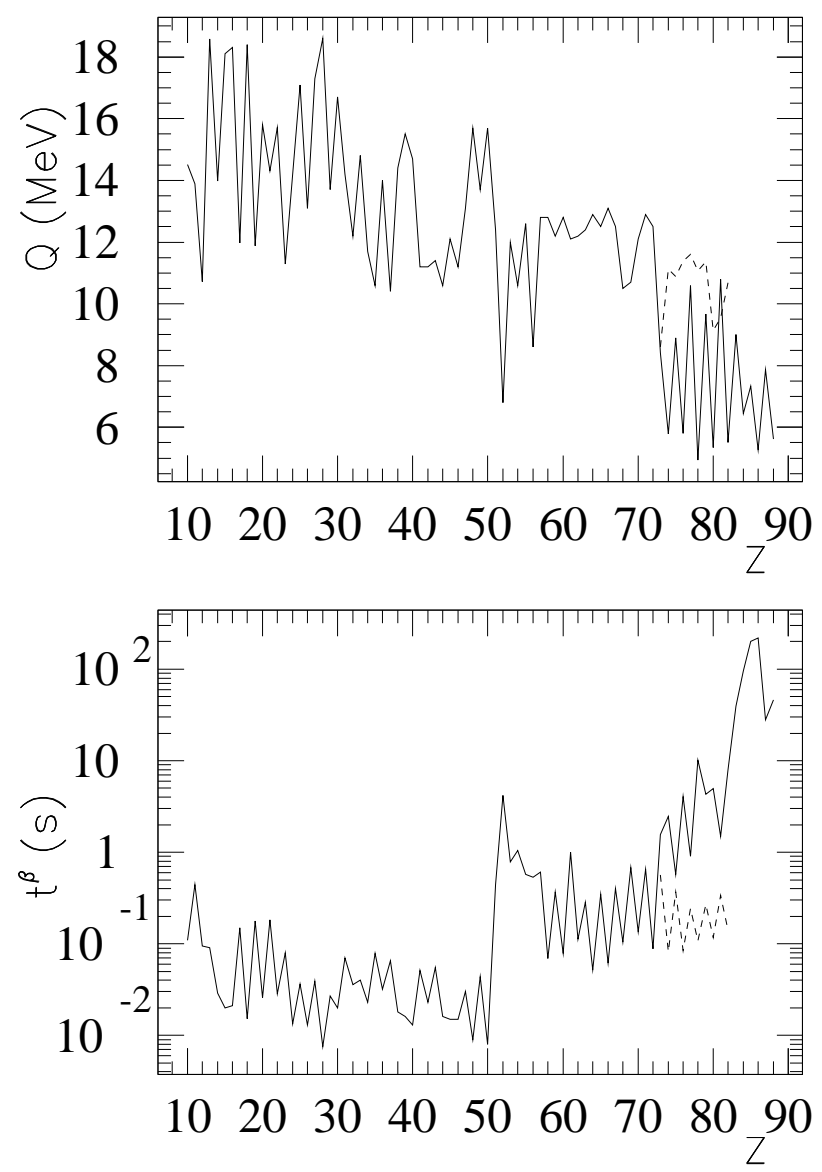

FIG. $14 Q_{E C}$-value (upper figure) and beta-decay halflife (lower figure) as a function of proton number $Z$ for the lightest nucleus for each element where beta-decay remains the dominating decay mode. Dashed lines (for $73 \leq Z \leq 82$ ) are the values at the proton dripline. Experimental values from (Achouri et al., 2006, Audi et al. 2003a b, Dossat et al. 2007) are completed by estimates based on (Möller et al. 1997).

allowed for many proton-rich nuclei and may seem energetically favoured for nuclei above $Z=50$ where the beta-daughter often has a positive $Q_{\alpha}$ value (see figure 15. However, this decay mode is mainly important in light nuclei: the only nucleus above mass 20 where the $\beta \alpha$ branching ratio gets above $1 \%$ is ${ }^{110} \mathrm{I}$. The $\beta \alpha$ process has in heavier nuclei mostly been observed just above closed shells similar to what is seen for ground state alpha decays. The competing process to delayed proton emission is therefore delayed gamma emission. The retardation from the Coulomb barrier will also be significant for protons, but the staggering of the proton dripline of course implies that there will be nuclei with sizable $P_{p}$ for most even $Z$-values. Turning to nuclei that lie within the "odd- $Z$ dripline" it appears, with our present incomplete experimental knowledge, that beta-delayed proton emission with $P_{p}$ above $1 \%$ with few exceptions occur in nuclei that are at most one or two nucleons away from the line. One may thus regard significant beta-delayed

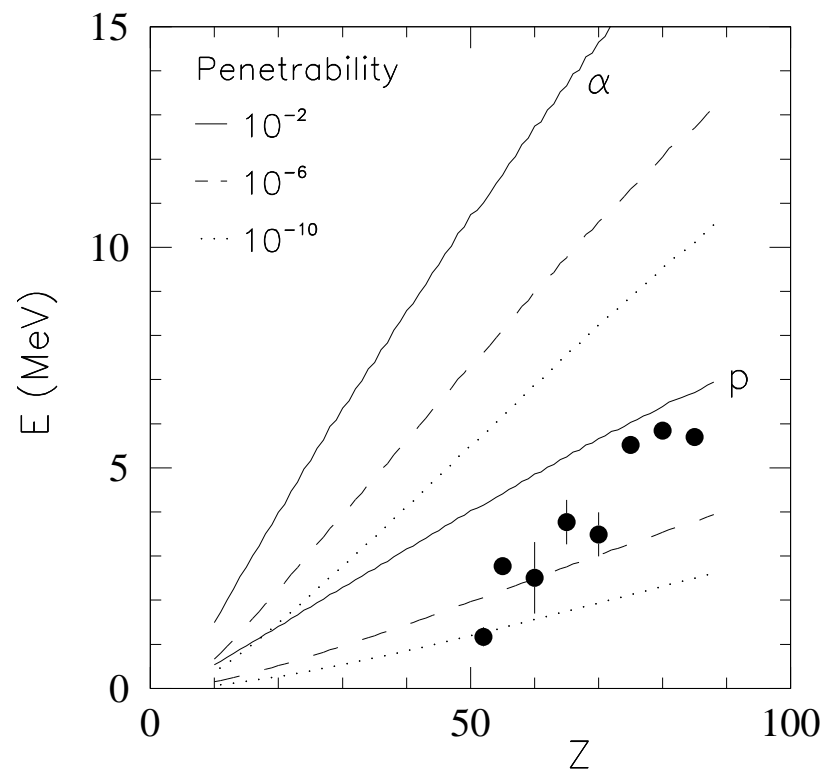

FIG. 15 The centre-of-mass energy that gives an s-wave penetrability of $10^{-2}$ (full lines), $10^{-6}$ (dashed lines) or $10^{-10}$ (dotted lines) for a beta-delayed proton or alpha particle are shown versus the charge $Z$ for the precursors shown in figure 14 Gamma emission can be expected to compete for penetrabilities below $10^{-6}$ (cf. figure 2). For illustration the emitter $Q_{\alpha}$ value (Audi et al. 2003b) is shown for a few beta-decaying nuclei, see the text.

proton emission as a dripline phenomenon. To give one example, the nucleus ${ }^{167} \mathrm{Ir}$ has a ground state and an isomer that both decay by proton emission, alpha particle emission and beta decay (Davids et al., 1997), but beta-delayed particle emission has not been reported even though the proton separation energy is below $2 \mathrm{MeV}$ in the daughter nucleus ${ }^{167}$ Os (however, such events may be harder to see with the tagging technique employed in the experiment).

The competition between proton and gamma emission can lead to the occurrence of gamma-delayed proton emission. The angular momentum barrier for outgoing protons seems to make this happen frequently in highspin physics (Rudolph, 2002), but it may also happen after beta decays where angular momentum barriers are smaller. It has been suggested to take place in the decays of ${ }^{20} \mathrm{Na}$ (Clifford et al., 1989 , Kirsebom, 2010) and ${ }^{31} \mathrm{Ar}$ (Wrede et al. 2009), but may be expected also in other nuclei. Gamma emission preceding particle emission is well-known in the light nuclei and recent dedicated reaction experiments have now succeeded to observe it even for cases where one or both of the unbound states are broad, namely for the $4^{+}$to $2^{+}$transition in ${ }^{8} \mathrm{Be}$ (Datar et al. 2005) and several transitions in ${ }^{12} \mathrm{C}$ (Kirsebom et al. 2009). 


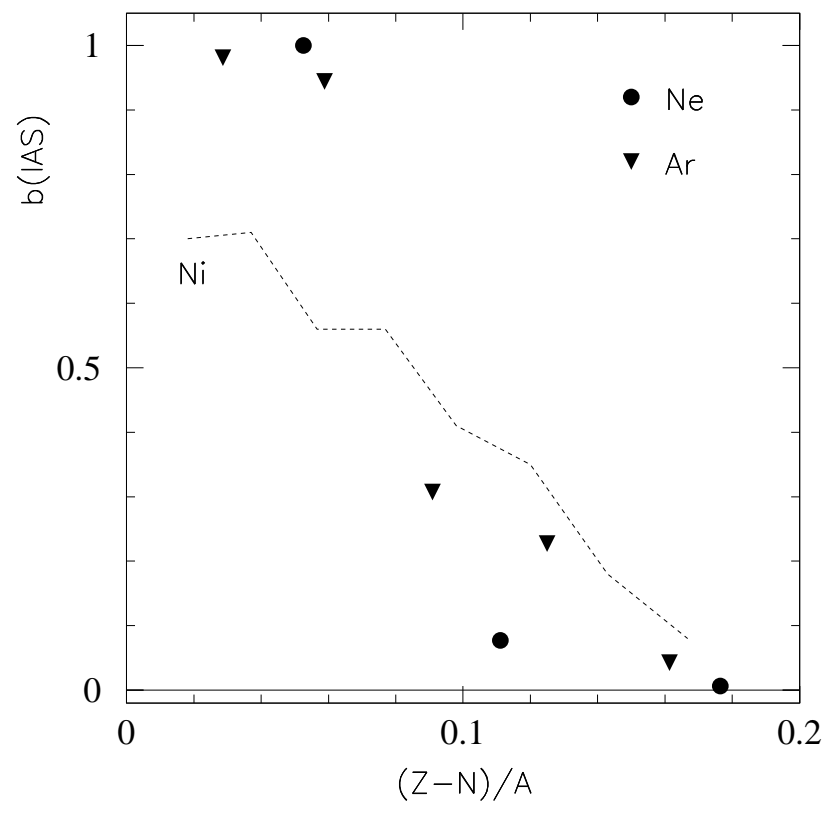

FIG. 16 The total branching ratio of beta-decays to the IAS is shown as a function of relative proton excess for the light $\mathrm{Ne}$ and Ar isotopes. The dashed line gives the estimated branching ratio for Fermi beta-decays to the IAS for the $\mathrm{Ni}$ isotopes. Only the Fermi part of the transition is included, the partial halflife is assumed to scale inversely with $Z-N$ and total halflives are taken from (Dossat et al., 2007).

\section{Fermi decays}

For $Z$ up to 50 the dripline nuclei have $N \leq Z$ so the Fermi strength contribute to beta decay. The approximate model-independence of $B_{F}$ makes the IAS transition interesting even though it, as shown in figure 16 . only dominates the decays close to $N=Z$ where the IAS is at low excitation energy. The decay rate for the transition to the IAS is proportional to $(Z-N) f_{\beta}\left(\Delta E_{C}\right)$ where the Coulomb energy shift $\Delta E_{C}$ depends only slowly on mass number for a given set of isotopes Antony et al. 1997). Even though this strength increases with $Z-N$ the branching ratio to the IAS will decrease. Furthermore since the IAS will be situated at higher and higher excitation energy its decay will become more fragmented and there will for the most proton-rich nuclei such as ${ }^{17} \mathrm{Ne}$ and ${ }^{31} \mathrm{Ar}$ not any longer be a dominant IAS peak in the final state spectrum.

A first approximation of the wavefunction of the IAS will be $|A\rangle$, the (normalized) state obtained by letting the isospin lowering operator work on the parent state. However, the two will not be exactly identical, a fact often referred to as isospin symmetry breaking. There are two aspects of this: the isospin of the IAS will not be pure (isospin mixing) and the radial wavefunctions may differ slightly (imperfect overlap, here isospin is in principle conserved). The magnitude of the combined effect has been an important issue in precision determina- tions of the Cabibbo-Kobayashi-Maskawa quark-mixing matrix element $V_{u d}$ (Towner and Hardy, 2010). Models predict increases from around $0.2 \%$ for ${ }^{10} \mathrm{C}$ to close to $2 \%$ for ${ }^{74} \mathrm{Rb}$ (Grinyer et al. , 2010) and it has been measured to $2.0(4) \%$ in ${ }^{32} \mathrm{Ar}$ (Bhattacharya et al., 2008) and can be expected to be of similar magnitude along the dripline. Isospin mixing is known from reaction studies to be small in the sense that the spreading width of an IAS typically is in the range 10 to $100 \mathrm{keV}$, see (Harney et al., 1986, Mitchell et al., 2010) and references therein. Note that many of the present beta-delayed proton experiments may not be able to resolve the IAS from close-by levels that it mixes with; the average level spacing can be estimated from the mirror systems where proton scattering on even-even nuclei give about $10 \mathrm{keV}$ for nuclei at mass 50 (Bilpuch et al. 1976).

To discuss the Fermi strength distribution in more detail one can start from the simple relation

$$
<A|H| A>=\sum_{i} E_{i}|<i| A>\left.\right|^{2},
$$

where $H$ is the Hamiltonian of the system and $\mid i>$ a complete set of states. Although $\mid A>$ is not an eigenstate of the system, the expectation value on the left is the quantity that formally enters in the isobaric multiplet mass quation (IMME). However, the IMME is very resilient (Benenson and Kashy, 1979, Bentley and Lenzi, 2007) and will hold in many cases even though isospin symmetry breaking may be significant in the intermediate multiplet members. The Fermi strength that is spread out via isospin mixing will obviously remain close to the IAS, the important consequence of eq. 11 being that this also holds on average for the Fermi strength spread out due to imperfect overlap between $|A\rangle$ and the IAS. The redistribution of Fermi strength has been checked experimentally in a few decays, e.g. ${ }^{20} \mathrm{Na}$ (Clifford et al. 1989), and it would be interesting to have thorough studies in more nuclei where the effects are expected to be large such as when continuum effects become important.

The particle emission from the IAS will in most cases be isospin forbidden (Auerbach, 1983, Brown, 1990) and the width will consequently be so narrow that gamma decays may have a substantial branching ratio. This is well established in light nuclei and must be kept in mind when detailed investigations of the Fermi strength become possible also in heavier nuclei.

\section{Gamow-Teller decays}

The GTGR will for proton-rich nuclei lie above the IAS, but can be reached in beta decay e.g. for the lightest Ar (Borge et al., 1989) and Ca (Trinder et al., 1997) isotopes allowing for experimental tests of the predicted strength distribution. The GTGR is predicted to be accessible even for $N=Z$ nuclei above mass 64 (Hamamoto 
and Sagawa, 1993). The experimental knowledge is still limited, but present data appear consistent with shellmodel calculations (Dossat et al. 2007). For nuclei with $N>Z$ the systematics of the Gamow-Teller strength is given in (Batist et al., 2010, Langanke and MartínezPinedo, 2000).

There is a special interest in the nuclear structure around the doubly magic nucleus ${ }^{100} \mathrm{Sn}$. This is the last particle stable $N=Z$ nucleus, the halflife has now been measured (Bazin et al., 2008) for all of them. For nuclei with $Z \leq 50$ and $50 \leq N$ allowed beta decay will mainly proceed via the $\pi g_{9 / 2} \rightarrow \nu g_{7 / 2}$ transition and for nuclei approaching ${ }^{100} \mathrm{Sn}$ all of the strength again appears to be accessible in beta decay. A comprehensive overview was given recently by Batist et al. (2010).

\section{Selected spectroscopic tools}

This subsection will present a few physics phenomena that can be employed to extract more detailed information on the states entering in beta-delayed particle emission, namely recoil shifts, interference between levels and decays where individual levels are not resolved.

To experimentally distinguish Fermi and GamowTeller transitions one may be guided by spin selection rules, but in general have to resort to beta recoil effects (Holstein, 1974). The beta-neutrino angular correlation will give a significantly larger recoil shift in Fermi transitions than in Gamow-Teller transitions and can be studied either as a function of beta-particle angle (Clifford et al. 1989) or through measurement of the peak shape (Schardt and Riisager, 1993). The size of the shift scales inversely with the mass number and is therefore easier to measure for light nuclei. It will depend on the spin sequences in the decay and has e.g. been used to determine the spin of ${ }^{31} \mathrm{Ar}$ (Thaysen et al. 1999).

The level density of nuclei increases with excitation energy and with mass number. As it increases the local structure changes from rather regular to essentially chaotic, a transition well-studied theoretically but experimentally less understood in nuclei (Weidenmüller and Mitchell, 2009). In many nuclei around mass 100 the beta-delayed proton spectra will be dominated by unresolved isolated resonances and fluctuation analysis is needed to extract information on the average spectral properties (Hansen et al., 1990) (see also Giovinazzo et al. (2000) for later work around mass 70). The larger windows for beta-delayed protons in lighter nuclei close to the driplines will enable these studies to be continued to cases with different level density. In the decays with highest $Q_{E C}$ values one may reach excitation energies close to where Ericsson fluctuations have been observed in nuclear reactions, i.e. the region where the level widths are larger than the average level distance. It will be experimentally challenging to look for such fluctuations in beta decay.

Another aspect of spectroscopy at high level density is that "complete spectroscopy" will be very challenging to achieve, see the discussion in Hansen et al. (1990). A way of overcoming this challenge is the total absorption technique (Janas et al., 2005, Rubio et al., 2005), where the aim is to measure the total emitted energy (apart from the emitted beta and neutrino particles) rather than the individual protons and gamma rays. This of course also holds for decays of neutron-rich nuclei where it, as demonstrated recently (Algora et al. 2010), is essential for a correct understanding of the decay heat in nuclear reactors.

A final effect that can influence decay spectra significantly is interference due to overlapping levels of the same spin and parity. This will occur not only at high excitation energy, but also for otherwise well-resolved states whose tails overlap. Interference will be clearly prominent in light nuclei where broad states occur frequently, but it will certainly be an issue also for the broad states that can appear for heavier nuclei due to width collectivization once the level density is so large that levels start to overlap, see (Celardo et al., 2008; Zelevinsky, 1996) and references therein. Interference effects needs a more careful theoretical treatment, e.g. via the R-matrix formalism. The effects are often easy to identify once the statistics is sufficient and may range from slight distortions, as in the beta-delayed proton spectrum from ${ }^{33} \mathrm{Ar}$ (Schardt and Riisager, 1993), to considerable spectral modifications, as in the beta-delayed alpha spectrum from ${ }^{18} \mathrm{~N}$ (Buchmann et al. , 2007). However, interference effects are not always easily recognizable, as seen in the beta-delayed alpha-decays of ${ }^{8} \mathrm{~B}$ (Barker, 1989), ${ }^{12} \mathrm{~N}$ (see subsection IV.D and to some extent also ${ }^{16} \mathrm{~N}$ (Buchmann et al. 2009), and when statistics is insufficient spectral features arising from broad and interfering levels are easily misinterpreted as new weak transitions as demonstrated e.g. for the beta-delayed proton spectrum from ${ }^{17} \mathrm{Ne}$ (Borge et al. 1988). This underlines the care that must be taken when interpreting decay spectra.

\section{C. $\beta^{-}$delayed emission of one particle}

\section{Occurrence of particle emission}

Figure 17 shows $Q_{\beta}$ and the beta-decay halflives along the neutron dripline. The values are theoretical estimates and will depend on the theoretical model chosen, in particular on how the model predicts the nuclear shell structure (Sorlin and Porquet, 2008) evolves. However, the following general observations are most likely robust. (As shown by Möller et al. (1997) their theoretical halflives agree better with experimental value the larger the $Q$ value is.)

The halflives for nuclei at the neutron dripline vary 

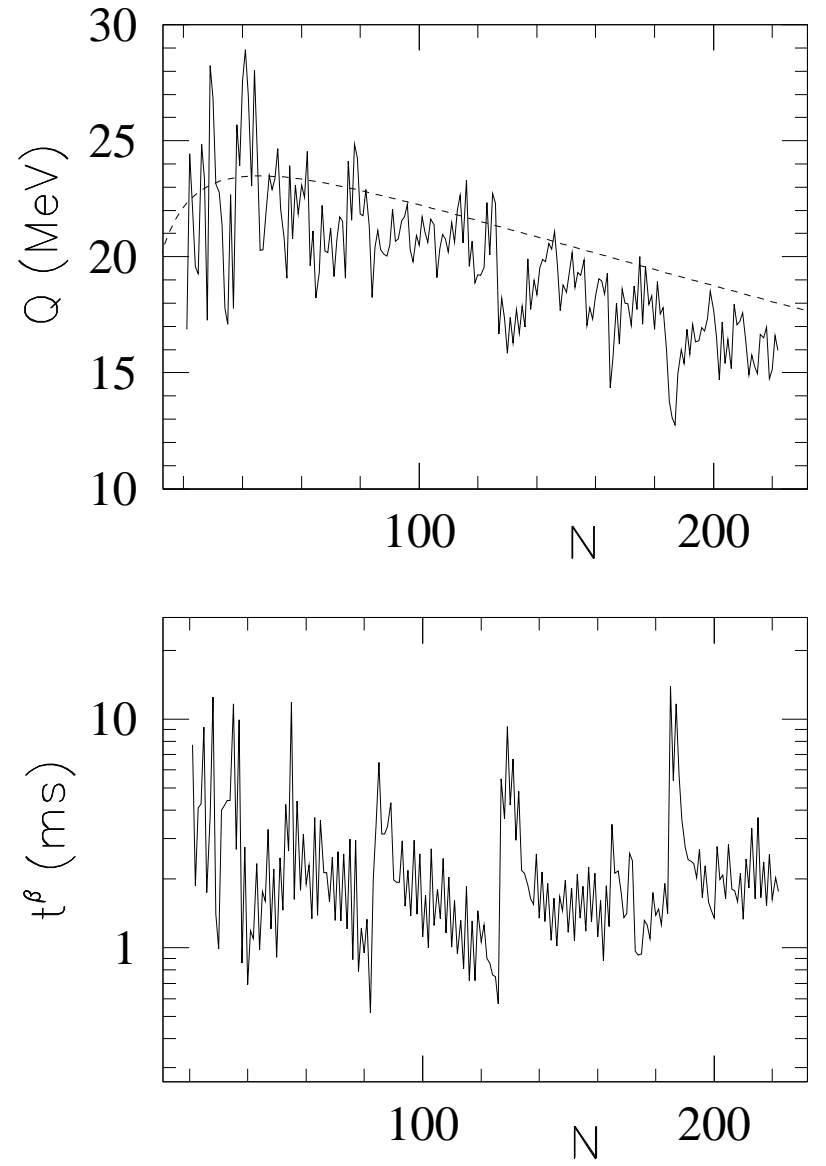

FIG. $17 Q_{\beta}$-value (upper figure) and beta-decay halflife (lower figure) as a function of neutron number $N$ for the lightest particle stable nucleus for a given $N$. The experimental dripline position is used for $N$ up to 30, all other values are taken from (Möller et al. , 1997, 2003). The dashed line gives the $Q$-values from an estimate based on the Weizsäcker mass formula.

somewhat for the experimentally known ones with $N<$ 30 , but are likely to mainly be in the range $1-3 \mathrm{~ms}$ once we get above $N$ about 40. Deviations will be due to changes in the $Q_{\beta}$-value rather than other structure effects. The $Q$-values may be affected by shell structure, but decrease slowly towards higher masses. This overall trend is seen already from the simplest possible liquid drop formula, as also indicated in the figure. An even smoother dependence of halflive with nucleon number is found in recent work based on the density functional approach (Borzov et al. 2008 ), but it is clear that both $Q$-values and halflives vary much less for neutron-rich nuclei than for the proton-rich ones.

Neutron emission will take place once it is energetically allowed and beta-delayed neutron emission will therefore be an important feature for neutron-rich nuclei. The extent is illustrated in figure 18 not only are beta-delayed multi-neutron decays energetically allowed shortly after beta-delayed one-neutron decay, the estimated beta strength distribution will soon give more than one emitted neutron on average per decay. As an example, for neutron dripline nuclei around mass 180 one expects more than 10 neutrons emitted in the decay chain towards beta-stability. Experimentally, we have today mainly reached this extended region of high $P_{n}$-values for the light nuclei.

Similar to what is observed for proton-rich nuclei the Coulomb barrier will limit significant beta-delayed alpha emission to the very light nuclei. However, the delayed emission of hydrogen isotopes, in particular deuterons and tritons, may also occur with small probabilities. Since their Q-values are limited, as seen from eq. 10, the deuteron emission will be suppressed by three orders of magnitude at mass 100 whereas triton emission may still be possible to see up to mass 200. Their physics relevance will be discussed shortly. The major difference to the situation for proton-rich nuclei is therefore the prominent beta-delayed one-neutron and multi-neutron emission.

The excitation energy of the Gamow-Teller Giant Resonance, that carries the main part of the Gamow-Teller strength, is known to decrease linearly with respect to the Isobaric Analogue State as a function of $(N-Z) / A$ (Langanke and Martínez-Pinedo, 2000, Osterfeld, 1992). It was pointed out by Sagawa et al. (1993) that the GTGR for light neutron-rich nuclei (oxygen or below) even could move below the initial state so that a major part of the strength can be accessed in beta-decay. For heavier nuclei, decays will take place to the tail of the GTGR.

\section{Decays in different mass regions}

To illustrate the present stage of the field, this section will present experimental results from several currently investigated mass regions, starting with the lightest nuclei which is where the neutron dripline is reached and halo structures (Jensen et al., 2004) have been studied.

Neutron halo nuclei must have low neutron separation energy and have a "clustered" structure in the sense that the halo neutrons should decouple from the core to a large extent. It is obvious from eq. 10 that betadelayed deuteron emission will be energetically favored in two-neutron halo nuclei, furthermore the component where the two halo neutrons decay to a deuteron (with the core as spectator) will give an important contribution to this decay mode. In fact, most theoretical calculations of the $\beta d$ decay only includes decays of the halo neutrons directly to continuum deuteron states. The early work on this decay mode is reviewed in Nilsson et al. (2000). The decay has so far only been seen in ${ }^{6} \mathrm{He}$ and ${ }^{11} \mathrm{Li}$ and the first experiments at ISOLDE have now been extended at other laboratories both for ${ }^{6} \mathrm{He}$ (Anthony et al. 2002 Raabe et al. 2009) and ${ }^{11} \mathrm{Li}$ (Raabe et al., 2008). The branching ratio is for ${ }^{6} \mathrm{He}$ now determined to be 


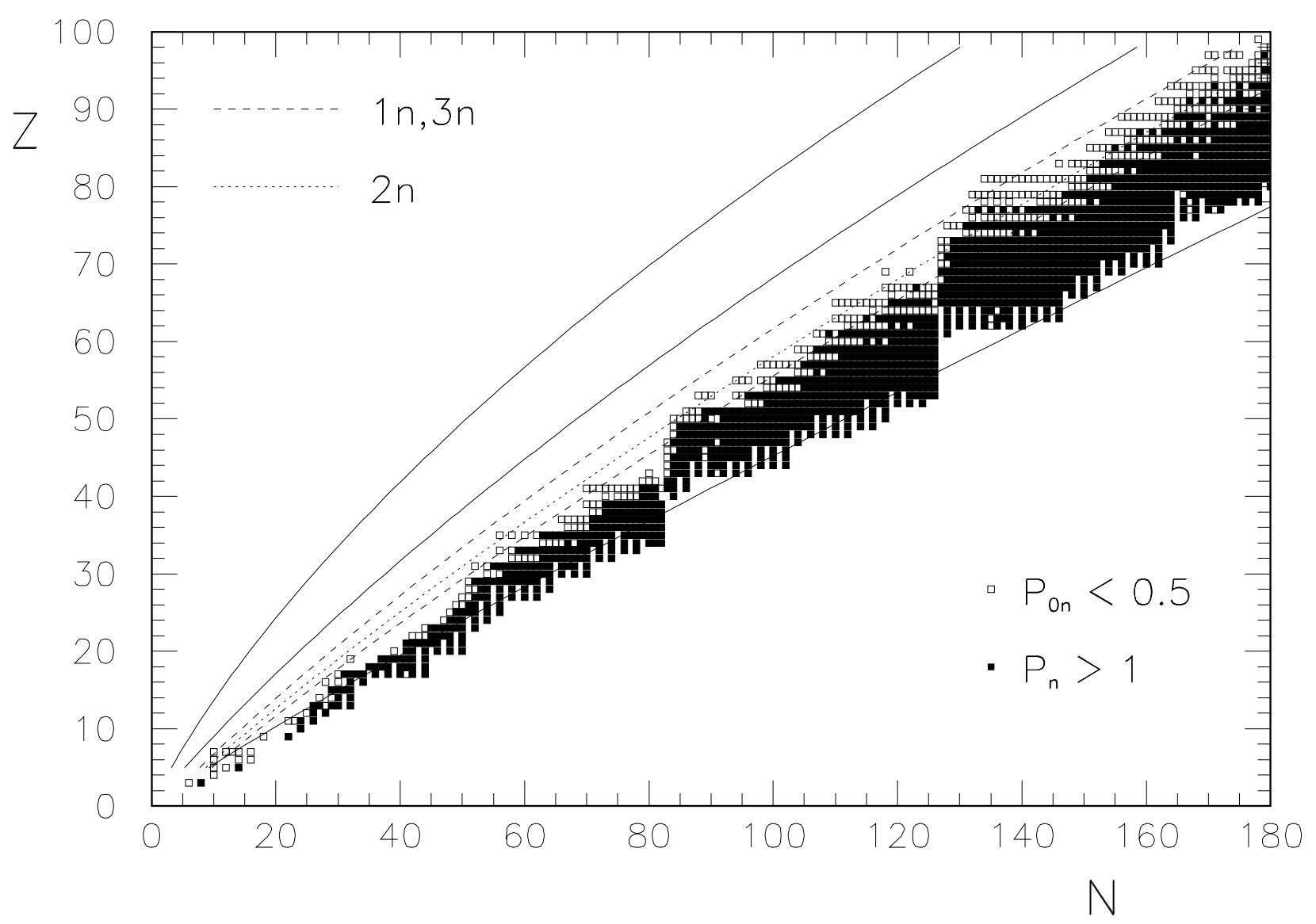

FIG. 18 Nuclei with large beta-delayed neutron-emission probability are marked with an open square if the probability for emitting one or more neutrons is larger than $50 \%$ and with a filled square if the average number of emitted neutrons is larger than one. The $P_{n}$ values are taken from experiment (Audi et al., 2003a, Borge et al. 1997, Yoneda et al. , 2003) for $N<20$ and from Möller et al. (2003) otherwise. The full lines indicate the line of beta-stability and the two driplines estimated from the Weizsäcker mass formula and the broken lines the corresponding estimates for where beta-delayed one-, two- and three-neutron emission becomes energetically allowed.

$1.65(10) \cdot 10^{-6}$ above a centre-of-mass energy of $525 \mathrm{keV}$. This very low value is understood to be due to cancellation in the matrix elements between contributions from small and large radii. The latest calculations (Tursunov et al. 2006a b reproduce both shape and intensity of the deuteron distribution, but it is not yet clear whether the theoretical and experimental maximum intensity positions agree, so measurements at lower energy would still be valuable. For ${ }^{11} \mathrm{Li}$ the branching ratio is $1.30(13) \cdot 10^{-4}$ above a centre-of-mass energy of $200 \mathrm{keV}$ and the spectrum is again rather featureless (Raabe et al. 2008$)$. The most recent theoretical calculations (Baye et al., 2006) give a qualitative agreement with data, but a real test of the theoretical understanding seems only possible once experimental data on the ${ }^{9} \mathrm{Li}+\mathrm{d}$ interaction at low energy are available.

The beta-delayed triton emission is again favoured at the neutron dripline and has been observed clearly in ${ }^{8} \mathrm{He}$ and ${ }^{11} \mathrm{Li}$ and at the $10^{-4}$ level in ${ }^{14} \mathrm{Be}$ (Jeppesen et al. 2002), but its relation to the structure of the emitting nucleus is less well understood. Recent experiments on ${ }^{11} \mathrm{Li}$ Madurga et al. 2009 ) and ${ }^{8} \mathrm{He}$ (Mianowski et al. 2010) have confirmed the decay mode with new experimental procedures, see figure 19, but it seems that more experimental data is needed before one can determine e.g. whether the triton decays proceed through states in the daughter nucleus or, as the deuterons, directly to the continuum. In the latter case the decay mode may depend on three-nucleon correlations in the decaying nucleus.

The decays of $A=9$ nuclei lead mainly to final states with two alpha particles and a nucleon. Complete kinematics decay studies have been performed on these nuclei during the last decade and have resulted in the discovery of new decay branches and in spin determination of several intermediate levels (Prezado et al. 2003,2005$)$. Strong Gamow-Teller branches in the mirror decays of ${ }^{9} \mathrm{Li}$ and ${ }^{9} \mathrm{C}$ go to states at around $12 \mathrm{MeV}$ excitation energy, but the deduced $B_{G T}$ values are large and a factor 4-5 larger for ${ }^{9} \mathrm{Li}$ than for ${ }^{9} \mathrm{C}$ which is not possible to understand from conventional theory (Kanada-En'yo, 2010, 

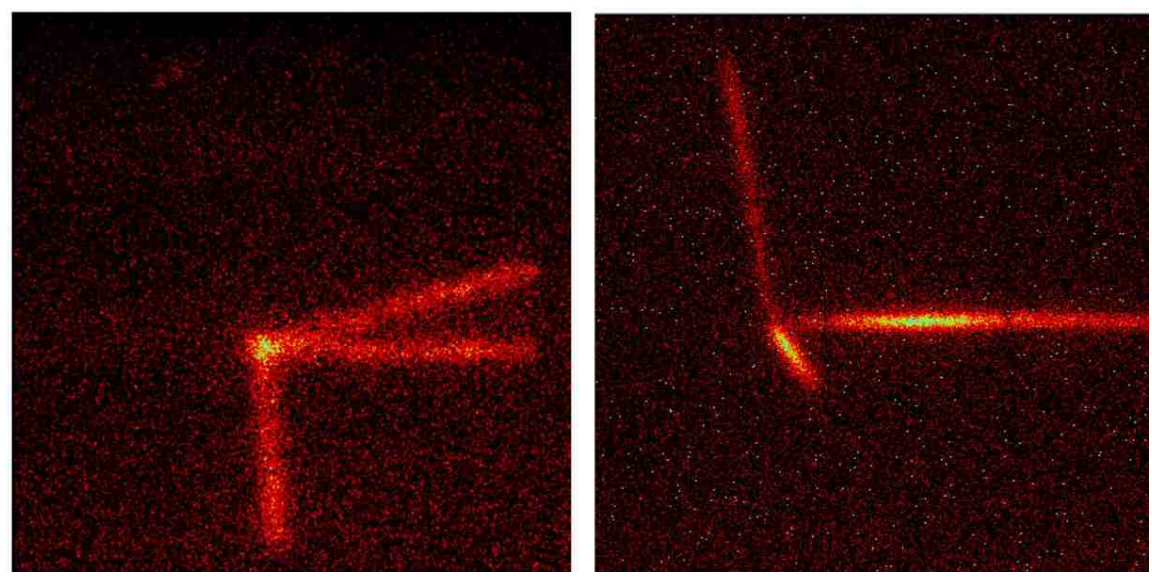

FIG. 19 Beta-delayed multi-particle decays recorded with the optical time projection chamber described in section III.C.1. The left panel shows beta-delayed three-proton emission from ${ }^{45} \mathrm{Fe}$ (from (Miernik et al., 2007b)) recorded so the incoming track is not visible, the right panel the track of a ${ }^{8} \mathrm{He}$ ion entering from the right that after beta-decay breaks up into a triton (long weak track), an alpha particle and an invisible neutron.

Millener, 2005). The reason for this is still unknown, the experimental strength (Prezado et al., 2003) may perhaps involve more than one level, and a proper theoretical investigation of the three-body continuum may help resolve the puzzle.

It would also be valuable to have calculations of the decays of the halo nuclei ${ }^{11} \mathrm{Li}$ and ${ }^{14} \mathrm{Be}$ that take the continuum into account explicitly. A more complete decay scheme for ${ }^{11} \mathrm{Li}$ is now available both at low (Mattoon et al. 2009) and high (Madurga et al., 2008) excitation energies in the daughter, but the deduced strength is still significantly less than that predicted by recent theory (Kanada-En'yo, 2010), in particular it has still not been possible to experimentally check the above mentioned prediction of the GTGR being placed below the initial state. A similar situation seems to be present for ${ }^{14} \mathrm{Be}$ where the experimental decay strength distribution (Jeppesen et al. , 2002) at high excitation energy is significantly lower than theoretical predictions. A better determination of the beta-delayed neutron branches could alleviate the problem, but may not suffice to solve it.

Much less is known about the decay of heavier dripline nuclei, but at least major beta-delayed neutron and/or gamma lines are known out to ${ }^{17} \mathrm{~B}$ (Raimann et al., 1996), ${ }^{19} \mathrm{C}$ (Ozawa et al., 1995$),{ }^{22} \mathrm{~N}$ (Sumithrarachchi et al. $2010)$ and ${ }^{24} \mathrm{O}$ (Reed et al. 1999$)$ and halflives and $P_{n}$ values are known for the heavier $\mathrm{B}, \mathrm{C}$ and $\mathrm{N}$ isotopes (Yoneda et al., 2003). In the region above oxygen the halflives are not known for the most neutron-rich isotopes of any element. The major decay branches are established for nuclei at a similar distance from the line of stability, e.g. for ${ }^{29} \mathrm{Ne}$ (Tripathi et al., 2006$)$ and ${ }^{33} \mathrm{Na}$ (Nummela et al. 2001, Radivojevic et al., 2002), but the dripline from here on is significantly further out (see section II).

Among the different physics questions that have been investigated in the heavier neutron-rich nuclei can be mentioned the stability of the $N=28$ shell that has been probed by extensive halflife measurements Grévy et al., 2004) as well as the $N=32$ and possible $N=34$ subshells probed in decays of $\mathrm{Sc}$ and Ti isotopes (Crawford et al. . 2010). The observed isotopic anomalies in some meteorites is known to depend on decay properties of very neutron-rich nuclei and motivated new measurements on the heavy Ar (Weissman et al. 2003) and Sc-Co (Sorlin et al., 2003) isotopes.

Recent experiments (Hosmer et al., 2010, Hosmer et al., 2005, Winger et al. 2009) have succeeded in determining halflives and $P_{n}$ values for ${ }^{78} \mathrm{Ni}$ and nuclei around it. Apart from the interest in settling the properties of this doubly magic nucleus the information is also needed to fine-tune calculations of the astrophysical $r$-process in this mass range where there is sensitivity in particular to the halflife of ${ }^{78} \mathrm{Ni}$ itself (Hosmer et al. 2010 ). At higher masses the nuclei participating in the $r$-process have been reached experimentally at $N=82$, see (Langanke and Martínez-Pinedo, 2003, Pfeiffer et al., 2001) for more details.

\section{Selected spectroscopic tools}

Beta-recoil effects may play a role for beta-delayed neutrons similar to that discussed above for beta-delayed protons, but has only been explored in a few cases such as ${ }^{9} \mathrm{Li}$ (Nyman et al. 1990). If the nucleus recoiling from neutron emission emits a gamma ray the latter will also be Doppler broadened provided the gammaemitting state is sufficiently short-lived. This has been used to improve the decay scheme of ${ }^{11} \mathrm{Li}$ (Fynbo et al. 2004, Mattoon et al. 2009), and is a valuable way to cross-check results from the sometimes complex neutron 


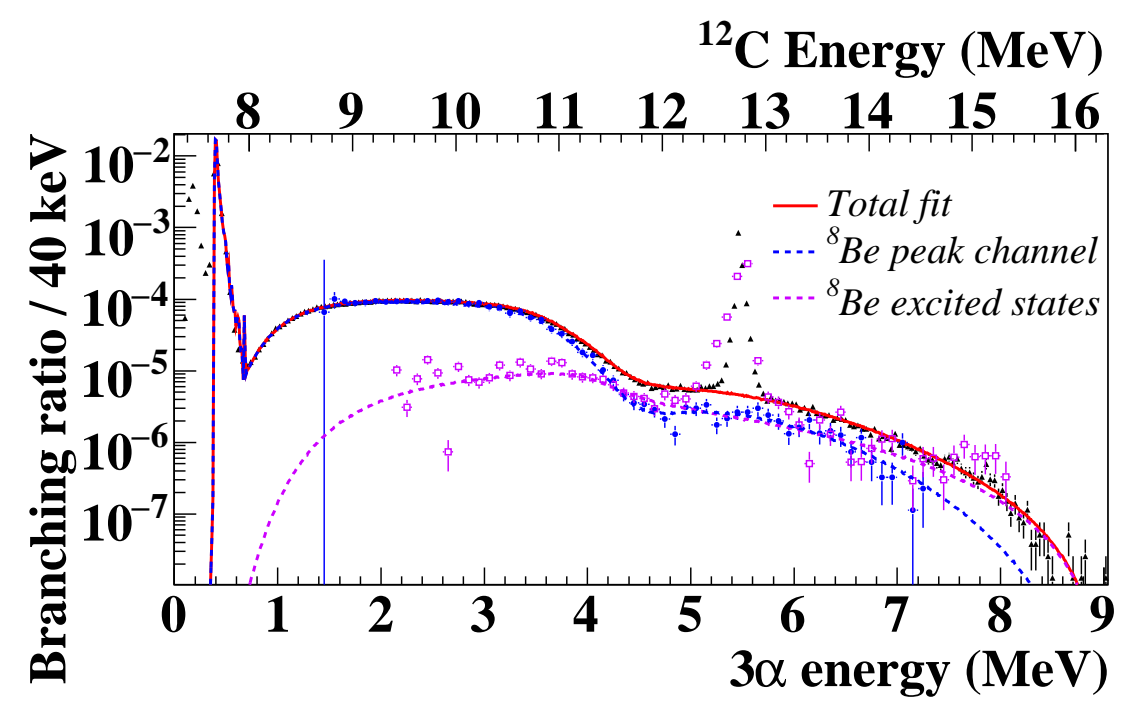

FIG. 20 (Color online) The branching ratio for beta-delayed alpha decay of ${ }^{12} \mathrm{~N}$ (filled triangles) is shown as a function of the total energy (Hyldegaard et al., 2010$)$. The solid line is a fit to the feeding to $0^{+}$and $2^{+}$states and does not include the contribution to the $1^{+}$state at $12.7 \mathrm{MeV}$. The filled circles (open squares) give the contribution from decays that do (do not) proceed through the ${ }^{8} \mathrm{Be}$ ground state, the dashed lines are the corresponding fits. See the text for details.

spectra and neutron-gamma coincidence measurements, see (Hirayama et al., 2005$)$ and references therein for ${ }^{11} \mathrm{Li}$. Analogous neutron-gamma experiments have already been performed e.g. for ${ }^{21} \mathrm{~N}$ (Li et al., 2009), ${ }^{33} \mathrm{Mg}$ and ${ }^{35} \mathrm{Al}$ (Angélique et al. 2006).

The analysis of decays through regions of high level density proceeds similarly to the case for the proton rich nuclei, except that the experimental challenges are higher due to the neutron detection. Fluctuation analysis will again be an important tool in order to extract reliable interpretations from beta-delayed neutron spectra (Hardy et al., 1978). For lighter nuclei where the level density is smaller one should in principle in the analysis of neutron spectra worry about exact lineshapes, interference effects etc. as was the case for the corresponding delayed proton spectra. However, most experiments presently determine neutron energies through time-of-flight and assume (at least implicitly) that their resolution will smear out such effects so that peaks in the spectra can be fitted with Gaussians. This practice could lead to wrong assignments.

\section{Beta delayed emission of several light particles}

Apart from decays through ${ }^{8} \mathrm{Be}$ and states in ${ }^{12} \mathrm{C}$ above the triple- $\alpha$ threshold (and a few weak transitions involving an $\alpha$ particle and a nucleon such as occurring in the decay of ${ }^{17} \mathrm{Ne}($ Chow et al., 2002$)$ ) beta-delayed emission of several light particles involves only nucleons. The first beta-delayed multi-nucleon decays, $\beta 2 \mathrm{n}$ and $\beta 3 \mathrm{n}$, were discovered about 30 years ago (Azuma et al. 1980 , 1979). The $\beta 2 \mathrm{p}$ process followed shortly after (Cable et al. 1983), but the $\beta 3$ p process was only observed a few years ago in ${ }^{45} \mathrm{Fe}$ (Miernik et al., 2007b) (see figure 19 and only recently also reported in ${ }^{43} \mathrm{Cr}$ (Pomorski et al. 2011a).

As shown in figure 18 beta-delayed multi-neutron emission will become dominant in the decays of very neutronrich nuclei, whereas the other processes only occur with small to moderate intensity (with the exception of the $A=8,9$ decays). Somewhat ironically, the multi-neutron process are the least studied ones, partly for experimental reasons due to the difficulty of neutron detection, partly due to the quite few cases of beta-delayed multi-neutron emission known today. The one case, ${ }^{17} \mathrm{~B}$, where betadelayed four neutron emission has been reported (Dufour et al. 1988) needs to be confirmed since other multineutron branches reported in the same work has since been shown to be too large (Bergmann et al. 1999 ).

The question of the particle emission mechanism is of prime importance. Although, as discussed in more detail in section VII, calculations of multi-particle final states in principle are becoming feasible now, it is still of interest to know whether simpler decay mechanisms, such as sequential decay, can describe a process or whether break-up directly into multi-particle continuum states takes place. Currently the only experimental information on beta-delayed multi-neutron emission comes from single neutron spectra (Azuma et al. 1979). There is more knowledge on $\beta 2 \mathrm{p}$ decays, as recently reviewed in (Blank and Borge, 2008). The most thoroughly studied case, that of ${ }^{31}$ Ar (Fynbo et al. 2000), seems to display only sequential emission and other $\beta 2 p$ cases are consistent with this. Turning to other beta-delayed multi-particles modes, the $\beta \alpha$ p decays from ${ }^{17} \mathrm{Ne}$ (Chow et al., 2002) 


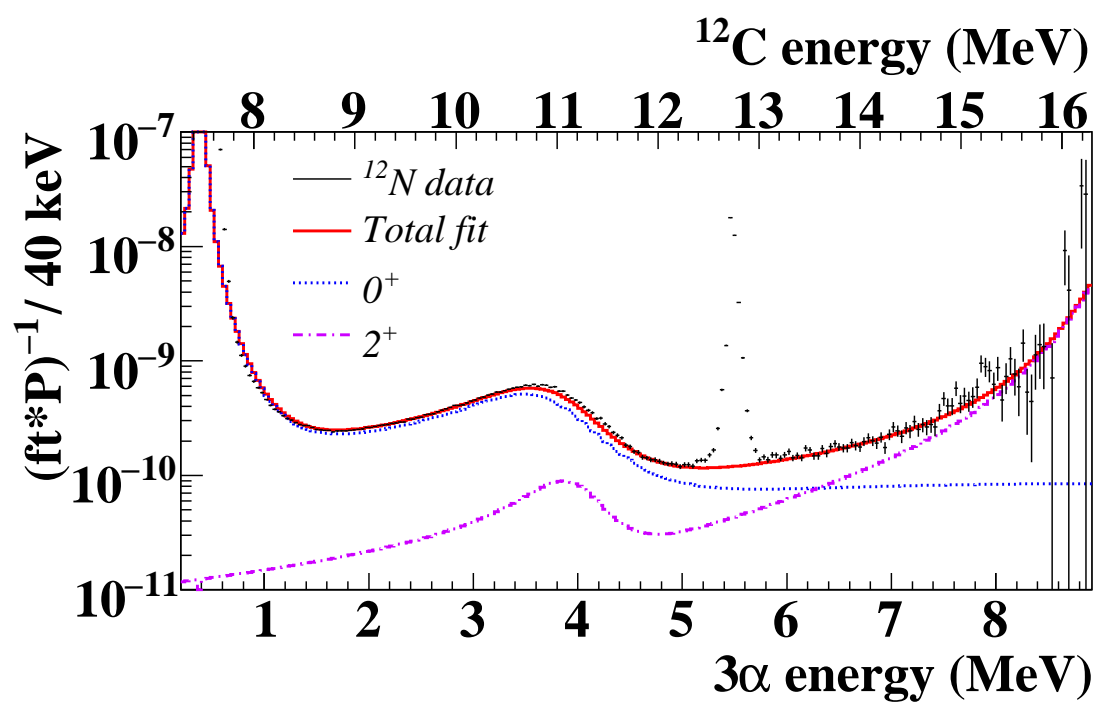

FIG. 21 (Color online) The beta-delayed alpha decay data for ${ }^{12} \mathrm{~N}$ shown in figure 20 are displayed corrected for the beta-decay phase space factor and alpha particle penetrability factors (from (Hyldegaard 2010)). The total fit is divided into contributions from $0^{+}$and $2^{+}$states in ${ }^{12} \mathrm{C}$. Note the clear interference between $0^{+}$states at low energy and the enhanced decay rate at high energy.

could also be analyzed assuming only sequential decays. For the light nuclei, a recent study (Madurga et al. 2008 ) of ${ }^{11} \mathrm{Li}$ indicated that most of the decays in the threebody $\left(\mathrm{n}+\alpha+{ }^{6} \mathrm{He}\right)$ and the five-body $(2 \alpha+3 \mathrm{n})$ channels are sequential and to a large extent proceeds through various He isotopes; a smaller direct break-up component is, however, still possible. Finally, for the $A=9$ decays mentioned above in section IV.C most decay branches can be approximately described in a sequential picture, but there are indications (Prezado et al. 2005) that there is a direct break-up component from the $5 / 2^{-}$level at $2.43 \mathrm{MeV}$ in ${ }^{9} \mathrm{Be}$. The hypothetical sequential branches through ${ }^{5} \mathrm{He}_{g s}$ and ${ }^{8} \mathrm{Be}\left(2^{+}\right)$, which give energy distributions that for the higher-lying broader states can be distinguished and seem to be observed, would give overlapping energy distributions for the $2.43 \mathrm{MeV}$ level. The sequential picture anyway does not really make sense in this situation as discussed in section VII. For the specific case of the $2.43 \mathrm{MeV}$ level the break-up mechanism has been investigated in three-body calculations (ÁlvarezRodríguez et al. 2008a) where the experimental energy and angular distributions could be reproduced.

The analysis of such data often makes use of the Rmatrix formalism since this allows for level parameters to be fitted to experiment. The adaptation of the formalism to beta decay is described e.g. in Barker and Warburton (1988). It is a priori applicable only for two-body decays, but has been employed in practice also for sequential decays due to the lack of better approaches. Robson (1975) has shown how to formally make sense of extensions of R-matrix to multi-particle situations, but this has not been implemented in data analysis. One can therefore not rely fully on results derived from present R-matrix fits.

It would be interesting to have more detailed data on the decay mechanism for $\beta 3 p$ decays. On a longer timescale it is without doubt the multi-neutron detection capabilities that constitute the key challenge for future progress in this field.

\section{The case of $A=12$}

The complications that may arise in beta-delayed decays can be illustrated with the case of ${ }^{12} \mathrm{~N}$ (and ${ }^{12} \mathrm{~B}$ ) whose decay into the $3 \alpha$ continuum has recently been studied in detail (Diget et al. 2009 , Hyldegaard et al. 2010, 2009) motivated by the importance of this continuum for the astrophysical triple- $\alpha$ process (Fynbo et al. 2005). The decay goes through narrow $1^{+}$states as well as through several $0^{+}$and $2^{+}$states that all couple strongly to the continuum. The experimental spectrum is shown in figure 20 as a function of the excitation energy in ${ }^{12} \mathrm{C}$. One can experimentally identify decays that proceed through the narrow ground state in ${ }^{8} \mathrm{Be}$, these decays are also marked in the figure and obviously correspond to sequential break-up. Apart from the Hoyle state at $7.65 \mathrm{MeV}$ all other states in the fit are broad. The resulting interference may be easier to see in figure 21 where the data have been corrected for the beta phasespace factor and the alpha particle penetrabilities. The interference actually also involves the upper tail of the Hoyle state (its "ghost", cf. Barker and Treacy (1962)) that owes its narrow width to a small value for the penetrability.

The increase in strength at higher energies seen for the 
$2^{+}$states is very hard to reconcile with the sum rule, eq. (9), if the decays are assumed to proceed through levels in ${ }^{12} \mathrm{C}$. This was taken (Hyldegaard et al. 2010) as an indication that some of the ${ }^{12} \mathrm{~N}$ decays proceed directly to continuum states. As mentioned when discussing figure 13 the standard assumption is that beta decays proceed through states in the emitter rather than directly into continuum states. Corresponding transitions directly to the continuum have been known for a long time for strong and electromagnetic processes (direct reactions and direct radiative capture, respectively), but are not generally recognized to occur also in weak decays. However, as mentioned in section IV.C it is the most natural explanation also for $\beta \mathrm{d}$ decays.

\section{E. Beta delayed fission}

Beta-delayed emission of particles heavier than alpha particles has only been seen as beta-delayed fission. An overview of this phenomenon with references to the early work can be found through Hall and Hoffman (1992); Kuznetsov and Skobelev (1999); and Shaughnessy et al. (2002). The probability for such decays depends both on the beta strength at high excitation energy and on the fission barriers (Möller et al. 2009), and the decay mode may therefore provide experimental information on fission in regions with high $Q_{\beta}$ values that is hard to obtain otherwise. Such information will enhance our understanding of the fission process and can help to determine better the role of fission in the $r$-process (MartínezPinedo et al., 2007).

Much of the recent activity has been driven by the continuous developments in radioactive beam production capabilities and has focussed on EC delayed fission in the light mass region. Experiments have been carried out on ${ }^{194} \mathrm{At}$ at GSI and on ${ }^{180} \mathrm{Tl}$ at ISOLDE (Andreyev et al. 2010). The latter experiment showed a surprising asymmetry in the mass distribution of the fragments. More detailed information should become available in the coming years.

\section{SINGLE-PROTON RADIOACTIVITY}

\section{A. Introduction}

The proton radioactivity is the process occurring in odd- $Z$ nuclei located beyond the proton-drip line. Due to the potential barrier (Coulomb and centrifugal) the emission of a proton from an unbound nucleus successfully competes with other forms of decays $\left(\beta^{+}, \alpha\right)$ only when the $Q_{p}$ value for the decay is sufficiently large, see Eq. 12. The proton radioactivity was discovered by Jackson et al. (1970) who observed protons emitted from an isomeric state in ${ }^{53} \mathrm{Co}$ at the excitation energy of $3.2 \mathrm{MeV}$. First observation of the ground-state proton radioactivity was reported 12 years later by Hofmann et al. (1982) for ${ }^{151} \mathrm{Lu}$ and by Klepper et al. (1982) for ${ }^{147} \mathrm{Tm}$. Presently, more then 40 proton emitters (from ${ }_{53}^{109} \mathrm{I}$ to ${ }_{83}^{185} \mathrm{Bi}$ ), including emission from long-lived isomeric states, have been established experimentally. Six of them $\left({ }^{131} \mathrm{Eu},{ }^{141 m} \mathrm{Ho},{ }^{141 g s} \mathrm{Ho},{ }^{144} \mathrm{Tm},{ }^{145} \mathrm{Tm},{ }^{146} \mathrm{Tm}\right)$ have transitions (so called fine structure) to the excited states in the respective daughter nuclei.

The importance of proton radioactivity follows from the fact that the knowledge of the decay energy and the half-life (width), combined with the relatively simple model of the potential barrier penetration, yields information on the nuclear wave function. Thus, relatively simple observables provide constrains on nuclear models for exotic nuclei, located beyond the proton drip-line. Since nuclear structure information is usually interpreted with help of the shell-model, it is convenient to divide proton emitters into two groups: those of the combined seniority one or two $(s \leq 2)$ and others with the combined seniority larger then two $(s>2)$. The combined seniority is defined as the number of unpaired nucleons (protons and neutrons). In the first case, apart from the odd-proton, and possibly an odd-neutron, no proton and neutron pairs are broken. Such decays are typical for ground states and for low lying isomers. In the $s=1$ case the odd proton can be pictured as moving in a singleparticle orbital in the nuclear potential of the even-even daughter nucleus, while the $s=2$ case corresponds to even-odd daughter with an odd neutron acting as a spectator. The majority of known proton emitters belong to this category, they will be discussed in Sec. V.B. The situation of $s>2$ corresponds to the proton emission from highly excited isomers having multiparticle character, which involves breaking additional pairs of protons or neutrons. In Sec. V.C we discuss a few known cases.

In addition to the information extracted directly from proton emission observables, nuclear structure information has been gathered by using the emitted protons as a tag in the recoil decay tagging (RDT) studies (Seweryniak et al. 2007b, 2001, Yu et al., 1998).

An overview of nuclear structure studies at the proton drip-line by means of proton radioactivity was given 
recently by Blank and Borge (2008). More detailed discussion of proton radioactivity was given by Woods and Davis (1997) and Hofmann (1995). The work of Sonzogni (2002) contains a compilation of results on proton emitters known in 2001.

\section{Fundamentals}

The necessary condition for a nuclide to decay by proton radioactivity is a positive decay energy $Q_{p}$ defined as the difference between binding energies (Eq. 12) of the parent and the daughter atoms:

$$
Q_{p}=B(N, Z-1)-B(N, Z)=-S_{p} .
$$

To separate the contribution from the atomic electrons, the decay energy is expressed in the form:

$$
Q_{p}=Q_{p}^{n u c}-E S,
$$

where $Q_{p}^{n u c}$ is the nuclear part of the decay energy. It is determined by the nuclear masses:

$$
Q_{p}^{n u c}=M^{n u c}(N, Z)-M^{n u c}(N, Z-1)-m_{p},
$$

where $m_{p}$ is the proton mass. The ES is the electron screening correction defined as the difference between total electron binding energies in the parent and the daughter nuclides:

$$
E S=B e(N, Z)-B e(N, Z-1) .
$$

In the above the electron binding energy in the hydrogen atom has been neglected. The value of the screening correction $E S$ can be calculated from the tabulated electron binding energies (Huang et al. , 1976), or estimated by a simple formula:

$$
E S=0.49+0.0144 \cdot Z^{1.6} \mathrm{keV} .
$$

The accuracy of this parametrization is better than $0.5 \%$ for $42<Z<75$ and drops to $1.6 \%$ for $Z=83$. No influence of the neutron number (isotopic effect) is taken here into account.

The decay energy is shared between the proton $E_{p}$ and the recoiling atom. Therefore, the measured kinetic energy of the emitted proton is given by:

$$
E_{p}=\frac{\left(M(N, Z-1)+m_{e}\right)}{m_{p}+M(N, Z-1)+m_{e}} Q_{p} .
$$

In addition, the angular momentum as well as parity conservation laws have to be satisfied:

$$
\begin{aligned}
\vec{I}_{i} & =\overrightarrow{I_{f}}+(\vec{l}+\vec{s}) \\
\pi_{i} \cdot \pi_{f} & =(-1)^{l}
\end{aligned}
$$

where, $\vec{I}_{i}$ and $\overrightarrow{I_{f}}$ are spins of the initial and final nuclear states respectively, $\vec{l}$ is the angular momentum of the emitted proton, $\vec{s}$ is the spin of the proton, $\pi_{i}, \pi_{f}$ are parities of the initial and final states, respectively.

\section{Probability of proton emission}

Relatively simple calculations of the proton emission lifetimes are based on the result obtained by Gurvitz and Kalbermann (1987) who analyzed the decay widths and shifts of quasistationary states in the quantum mechanical two-potential approach. By investigating the quasiclassical limit they provided simple formulae which are similar to, but more general than, those achieved in the framework of WKB approximations (Brink et al. 1983). In this approach the width of the proton-emitting state is given by:

$$
\begin{aligned}
\Gamma_{p} & =S_{p} \frac{N}{4 \mu} \exp \left[-2 \int_{r_{2}}^{r_{3}} k(r) d r\right] \\
k(r) & =\left(2 \mu\left|Q_{p}^{n u c}-V(r)\right|\right)^{1 / 2},
\end{aligned}
$$

where the normalization factor $N$ has to satisfy the equation:

$$
N \int_{r_{1}}^{r_{2}} \frac{d r}{2 k(r)}=1
$$

$S_{p}$ is the spectroscopic factor described later and $\mu$ is the reduced mass of the proton and the daughter nucleus. Integration limits $r_{i}$ are the classical turning points, defined by $V\left(r_{i}\right)=Q_{p}^{n u c}$, where $V(r)$ is the radial part of the nucleus-proton potential, see Fig. 22 .

To simplify calculations, some authors replace the factor $\frac{N}{4 \mu}$ by the so called frequency of assaults factor $\nu$ Hofmann, 1996) calculated for the case of an $s$-wave proton leaving the square well plus Coulomb potential (Bethe, 1937)

$$
\nu=\frac{\sqrt{2} \pi^{2}}{\mu^{\frac{3}{2}} R_{c}^{3} \sqrt{(Z-1) e^{2} / R_{c}-Q_{p}^{n u c}}},
$$

where $R_{c}$ is the channel radius $R_{c}=r_{n u c} \cong r_{0}(A-1)^{1 / 3}$ with $r_{0}=1.21 \mathrm{fm}$. For example, in the decay of ${ }^{151} \mathrm{Lu} \nu$ equals $4.1 \cdot 10^{21} \mathrm{~s}^{-1}$.

Then, the proton emission decay constant is calculated as:

$$
\lambda_{p}=S_{p} \nu \exp \left[-2 \int_{r_{2}}^{r_{3}} k(r) d r\right]
$$

The potential $V(r)$ is taken as a superposition of nuclear $\left(V_{N}\right)$, coulomb $\left(V_{C}\right)$, centrifugal $V_{L}$, and spin-orbit $V_{l s}$ terms. The nuclear part is usually described by the Woods-Saxon form with various parametrization:

$$
V_{N}(r)=-V_{r} \frac{1}{1+\exp \frac{r-R}{a}} .
$$

Comparison between different parametrizations was done in the work of Ferreira et al. (2002). Although not supported by the work of Ferreira et al. (2002), the most frequently used parametrization is that of Becchetti and 


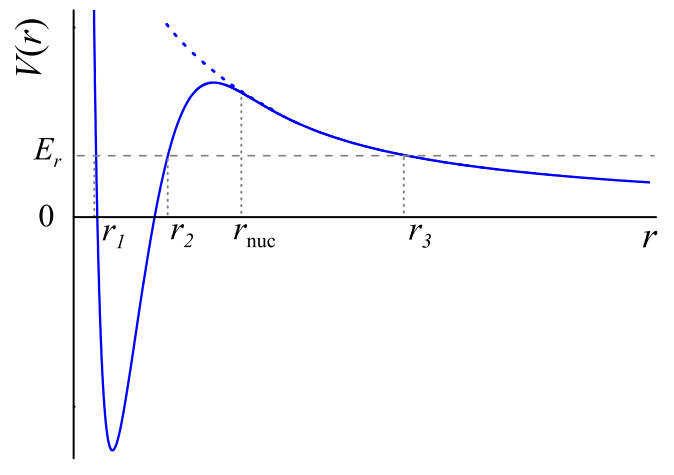

FIG. 22 Schematic view of the radial part of the nucleusproton potential. The classical turning points $r_{i}$ for a particle with energy $E_{r}$ are marked. The nuclear contribution turns to zero around $r=r_{\text {nuc }}$.

Greenlees (1969). Detailed potential descriptions and calculations for some of the emitters can be found in Áberg et al. (1997); Buck et al. (1992); and Hofmann (1995).

As an example, the half-life for the proton emission from ${ }^{151} \mathrm{Lu}$ as a function of the decay energy, for three values of the orbital angular momentum is shown in Fig. 23. The characteristic strong dependence on the available energy and the angular momentum is clearly seen.

\section{Spectroscopic factor $S_{p}$}

The spectroscopic factor $S_{p}$ is a measure of the singleparticle purity of the initial wave function. Within the BCS theory the spectroscopic factor is given by $S_{p}^{t h}=u_{j}^{2}$, where the vacancy factor $u^{2}$ is the probability that the spherical shell-model orbital with $(n, l, j)$ quantum numbers is empty in the daughter nucleus. For some proton emitters the factors $u_{j}^{2}$ can be found in the work of Áberg et al. (1997). This theoretical value is compared with the experimental value $S_{p}^{\exp }$ derived as the ratio of the measured partial decay constant and the calculated one assuming $S_{p}=1$. The agreement between the two values indicates that the correct assumption about the initial wave function has been taken. For example, in the case of ${ }^{151} \mathrm{Lu} S_{p}^{\text {exp }}=0.5$ and $S_{p}^{t h}=0.54$ assuming the proton was emitted from the $\left[\pi h_{11 / 2}\right] 11 / 2^{-}$state. The good agreement supports such an interpretation. In contrast, for the case of ${ }^{145} \mathrm{Tm} S_{p}^{\exp }=0.48$ and $S_{p}^{t h}=0.65$, which suggests that the spherical potential used in the calculation may not be applicable. Indeed, the deformed nature of ${ }^{145} \mathrm{Tm}$ was confirmed experimentally (Karny et al., 2003, Seweryniak et al., 2007a) and theoretically (Arumugam et al. 2008).

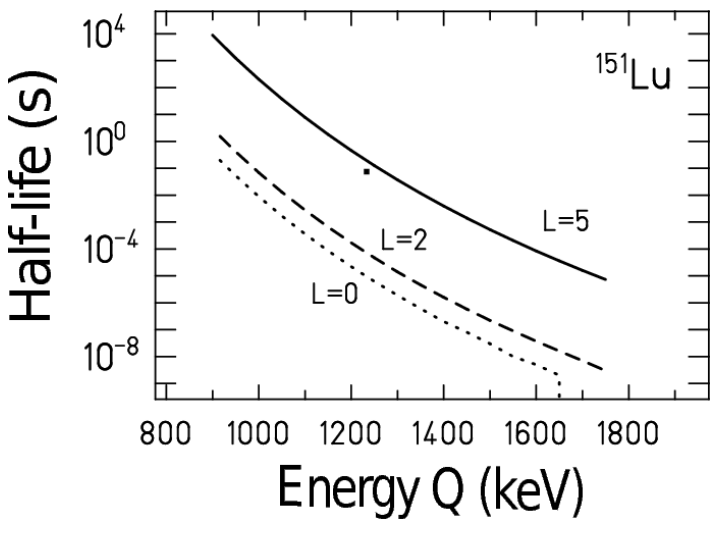

FIG. 23 The half-life for the proton emission as a function of nuclear decay energy $Q_{p}^{n u c}$ and the orbital angular momentum carried away by the proton. Calculations were done for the case of ${ }^{151} \mathrm{Lu}$ with $\mathrm{S}_{p}=0.54$. The measured values of the decay energy and the half-life, indicated by the black square, suggest the transition with $L=5$.

\section{Models of proton emission}

The usefulness as well as limitations of simple models, introduced in previous sections, may be illustrated with the example of ${ }^{145} \mathrm{Tm}$. In this nucleus two proton transitions from the same state have been observed (Karny et al. . 2003). The proton energies are $E_{p}=1.73 \mathrm{MeV}$ and $E_{p}=1.40 \mathrm{MeV}$, while the corresponding partial half-lives are $3.4 \mu \mathrm{s}$ and $32 \mu \mathrm{s}$. The first transition is interpreted as a decay to the $0^{+}$ground state of ${ }^{144} \mathrm{Er}$, while the second goes to the first excited $2^{+}$state in this nucleus. In the frame of the spherical quasi-classical approach we may assume that the first transition originates from the $\pi h_{11 / 2}$ orbital $(l=5)$ while the second from the $\pi f_{7 / 2}(l=3)$ component of the initial wave function. The calculations, including theoretical spectroscopic factors $\left(u^{2}\left(h_{11 / 2}\right)=0.647\right.$ and $\left.u^{2}\left(f_{7 / 2}\right)=0.985\right)$ yield the partial half-lives of $2.29 \mu \mathrm{s}$ and $1.28 \mu \mathrm{s}$, for the two transitions, respectively. Thus, by comparing with the experimental values, we may conclude that the emitter wave function consists of $67 \%=\frac{2.29}{3.4} \cdot 100 \%$ of the $l=5, \pi h_{11 / 2} \otimes 0^{+}$and $3.7 \%$ of $l=3, \pi f_{7 / 2} \otimes 2^{+}$components. The remaining $29 \%$ of the wave function does not participate in proton emission and therefore can not be determined within this simple model. The more elaborate coupled channel model which takes into account the dynamic deformation (Hagino, 2001; Karny et al., 2003) yields values of $56 \%$ for $\pi h_{11 / 2} \otimes 0^{+}$state and $\approx 3 \%$ for $\pi f_{7 / 2} \otimes 2^{+}$state.

Although simple models are useful for the first order approximations, they cannot be expected to yield correct results for highly deformed nuclides. For those cases more elaborate theoretical approaches have to be applied. Examples of such approaches are given by Áberg et al. (1997); Esbensen and Davids (2001); Fiorin et al. (2003); and Kruppa and Nazarewicz (2004). Calcula- 
TABLE III Summary of literature data for $s=1$ proton emitters. Empty place means no data available. For isotopes with ground and isomeric state emission a combined literature is given. In 'Reference' column additional letter E - refers to experimental papers, while letter $\mathrm{T}$ - points to the theoretical papers where properties of the referred nuclei are explicitly calculated.

\begin{tabular}{|c|c|c|c|c|c|c|}
\hline Emitter & Cross sec. & $\mathrm{E}_{p}(\mathrm{MeV})$ & $\mathrm{Q}_{p}^{\text {nucl }}(\mathrm{MeV})$ & $\mathrm{T}_{1 / 2}$ & Ang.mom. & References \\
\hline${ }_{53}^{109} \mathrm{I}$ & $40 \mu \mathrm{b}$ & $0.8126(40)$ & $0.829(4)$ & $93.5(3) \mu \mathrm{s}$ & $\mathrm{l}=2$ & 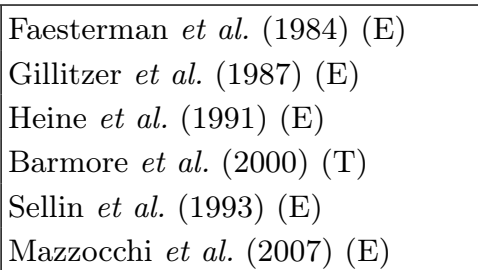 \\
\hline${ }_{55}^{112} \mathrm{Cs}$ & $0.5 \mu \mathrm{b}$ & $0.807(7)$ & $0.815(7)$ & $0.5(1) \mathrm{ms}$ & $1=2$ & $\begin{array}{l}\text { Page et al. }(1994)(\mathrm{E}) \\
\text { Ferreira and Maglione }(2001)(\mathrm{T})\end{array}$ \\
\hline${ }_{55}^{113} \mathrm{Cs}$ & $30 \mu \mathrm{b}$ & & $0.9771(37)$ & $16.7(7) \mu \mathrm{s}$ & $\mathrm{l}=2$ & 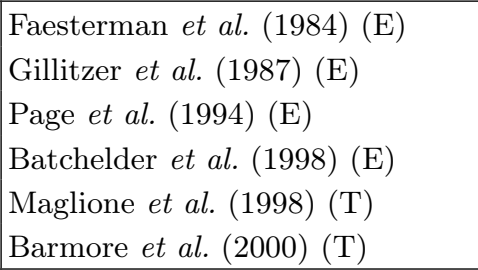 \\
\hline${ }_{57}^{117} \mathrm{La}$ & $240 \mathrm{nb}$ & $0.806(5)$ & $0.813(5)$ & $24(3) \mathrm{ms}$ & $1=2$ & $\begin{array}{ll}\text { Soramel et al. } & (2001) \\
\text { Mahmud et al. } & \text { (2001) (E) }\end{array}$ \\
\hline${ }_{59}^{121} \mathrm{Pr}$ & $0.3 \mathrm{nb}$ & $0.882(10)$ & $0.900(10)$ & $10_{-3}^{+6} \mathrm{~ms}$ & $l=4$ or 5 & $\begin{array}{ll}\text { Bogdanov et al. } & (1973)(\mathrm{E}) \\
\text { Robinson et al. } & (2005)(\mathrm{E})\end{array}$ \\
\hline${ }_{63}^{130} \mathrm{Eu}$ & $9 \mathrm{nb}$ & $1.020(15)$ & $1.028(15)$ & $0.90_{-0.29}^{+0.49} \mathrm{~ms}$ & $\mathrm{l}=2$ & $\begin{array}{l}\text { Mahmud et al. (2002) (E) } \\
\text { Davids } \text { et al. }(2004) \text { (E) }\end{array}$ \\
\hline${ }_{63}^{131} \mathrm{Eu}$ & $90 \mathrm{nb}$ & $\begin{array}{l}0.932(7) \\
811(7)^{\mathrm{a}}\end{array}$ & & $17.8(19) \mathrm{ms}$ & $\begin{array}{l}l=2 \\
l=2\end{array}$ & 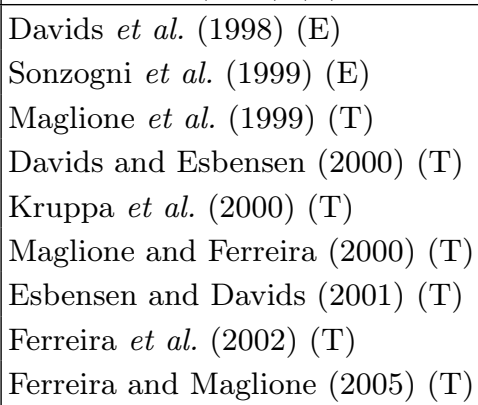 \\
\hline${ }_{65}^{135} \mathrm{~Tb}$ & $6 \mathrm{nb}$ & $1.179(7)$ & $1.188(7)$ & $0.94_{-0.22}^{+0.33} \mathrm{~ms}$ & $1=5$ & Woods et al. (2004) (E) \\
\hline${ }_{67}^{140} \mathrm{Ho}$ & $13 \mathrm{nb}$ & $1086(10)$ & & $6(3) \mathrm{ms}$ & $l=5$ & \begin{tabular}{|l|l} 
Rykaczewski et al. & (1999) (E) \\
Maglione and Ferreira & $(2002)$ \\
Ferreira and Maglione & $(\mathrm{T})$ \\
\end{tabular} \\
\hline \begin{tabular}{|c|}
${ }_{67}^{141} \mathrm{Ho}$ \\
${ }_{67}^{141} m$ \\
${ }^{2} \mathrm{Ho}$
\end{tabular} & $1.4 \mu \mathrm{b}$ & $\begin{array}{c}1169(8) \\
968(8)^{a} \\
1235(9) \\
1031(11)^{a}\end{array}$ & & $\begin{array}{l}7.4(3) \mu \mathrm{s} \\
7.4(3) \mu \mathrm{s}\end{array}$ & $\begin{array}{l}l=5 \\
l=3 \\
l=0 \\
l=2\end{array}$ & 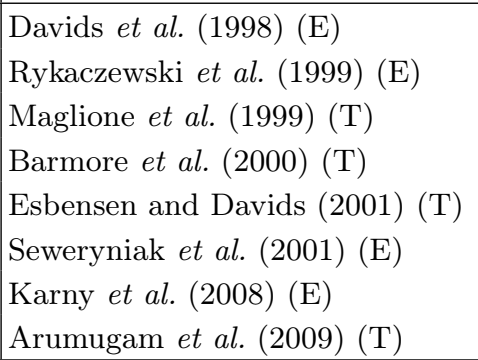 \\
\hline${ }_{69}^{144} \mathrm{Tm}$ & $10 \mathrm{nb}$ & \begin{tabular}{|c|}
$1700(16)$ \\
$1430(25)^{b}$
\end{tabular} & & $1.9_{-0.5}^{+1.2} \mu \mathrm{s}$ & $\begin{array}{l}l=5 \\
l=2^{b}\end{array}$ & \begin{tabular}{|l|l} 
Grzywacz et al. & $(2005)$ \\
Bingham et al. & $($ (E) \\
\end{tabular} \\
\hline
\end{tabular}

\footnotetext{
a transitions to the excited states
}

b evidence of the transition to the excited state based on two counts 
TABLE IV Summary of literature data for $s=1$ proton emitters. Continuation of Table III

\begin{tabular}{|c|c|c|c|c|c|c|}
\hline Emitter & Cross sec. & $\overline{\mathrm{E}_{p}(\mathrm{MeV})}$ & $\mathrm{Q}_{p}^{\text {nucl }}(\mathrm{MeV})$ & $\overline{\mathrm{T}_{1 / 2}}$ & Ang.mom. & References \\
\hline${ }_{69}^{145} \mathrm{Tm}$ & $0.5 \mu \mathrm{b}$ & $\begin{array}{l}1728(10), \\
1398(10)\end{array}$ & & $3.1(2) \mu \mathrm{s}$ & $\begin{array}{l}l=5 \\
l=3\end{array}$ & 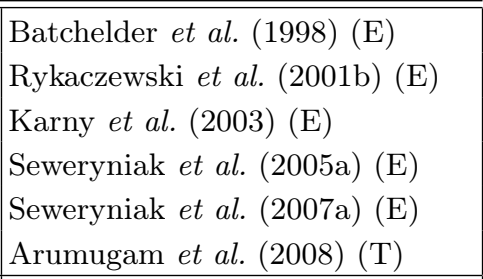 \\
\hline $\begin{array}{r}146 \mathrm{Tm} \\
146 m \\
69 \\
\mathrm{Tm}\end{array}$ & $1 \mu \mathrm{b}$ & $\begin{array}{c}1191(1) \\
1016(4)^{a} \\
889(8)^{a} \\
1120(1)^{a}\end{array}$ & & $200 \mathrm{~ms}$ & $\begin{array}{l}l=5 \\
l=3 \\
l=3 \\
l=5\end{array}$ & 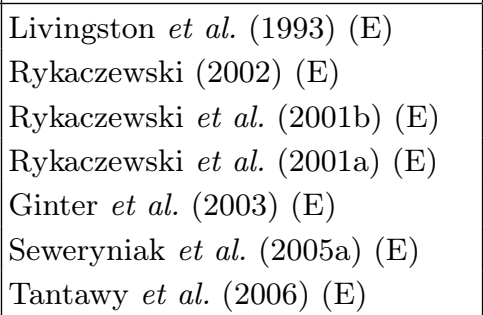 \\
\hline $\begin{array}{c}{ }_{69}^{147} \mathrm{Tm} \\
147 m \\
69 \\
\mathrm{Tm}\end{array}$ & $30 \mu \mathrm{b}$ & $1110.8(3.9)$ & $\begin{array}{l}1071.4(3.3) \\
1139.3(5.3)\end{array}$ & $\begin{array}{l}560(40) \mathrm{ms} \\
360(40) \mu \mathrm{s}\end{array}$ & $\begin{array}{l}\mathrm{l}=5 \\
\mathrm{l}=2\end{array}$ & 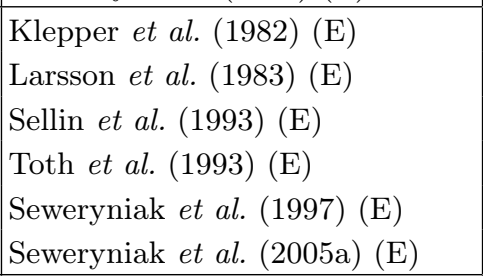 \\
\hline $\begin{array}{c}{ }_{71}^{150} \mathrm{Lu} \\
150 \mathrm{~m} \\
{ }_{71} \mathrm{Lu}\end{array}$ & $3 \mu \mathrm{b}$ & $\begin{array}{l}1261(4) \\
1286(6)\end{array}$ & & $\begin{array}{l}46(5) \mathrm{ms} \\
39_{-6}^{+8} \mu \mathrm{s}\end{array}$ & $\begin{array}{l}\mathrm{l}=5 \\
\mathrm{l}=2\end{array}$ & 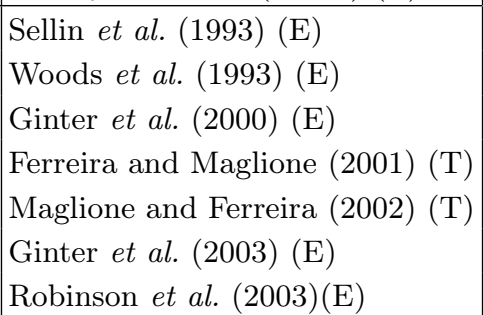 \\
\hline \begin{tabular}{|l}
${ }_{71}^{151} \mathrm{Lu}$ \\
${ }^{151 m} \mathrm{Lu}$
\end{tabular} & $70 \mu \mathrm{b}$ & $\begin{array}{c}1232.9(2.0) \\
1310(10)\end{array}$ & & $\begin{array}{l}80(2) \mathrm{ms} \\
16(1) \mu \mathrm{s}\end{array}$ & $\begin{array}{l}\mathrm{l}=5 \\
\mathrm{l}=2\end{array}$ & $\begin{array}{l}\text { Hofmann et al. }(1982) \text { (E) } \\
\text { Sellin } \text { et al. }(1993) \text { (E) } \\
\text { Yu et al. }(1998) \text { (E) } \\
\text { Bingham et al. } \quad(1999)(\mathrm{E}) \\
\text { Ferreira and Maglione }(2000)(\mathrm{T})\end{array}$ \\
\hline${ }_{73}^{155} \mathrm{Ta}$ & & $1444(15)$ & & $2.9_{-1.1}^{+1.5} \mathrm{~ms}$ & $l=5$ & \begin{tabular}{|l|l|} 
Uusitalo et al. & $(1999)(\mathrm{E})$ \\
Page et al. & $(2007)(\mathrm{E})$ \\
Joss et al. & $(2006)$ \\
\end{tabular} \\
\hline \begin{tabular}{|c|}
${ }_{73} \mathrm{Ta}$ \\
$156 m \mathrm{Ta}$ \\
${ }_{73} \mathrm{Ta}$
\end{tabular} & $50 \mathrm{nb}$ & $\begin{array}{l}1007(5) \\
1108(8)\end{array}$ & & \begin{tabular}{|l|}
$144(24) \mathrm{ms}$ \\
$375(54) \mathrm{ms}$
\end{tabular} & $\begin{array}{l}l=2 \\
l=5\end{array}$ & 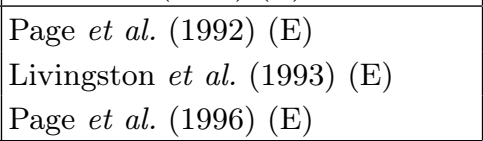 \\
\hline${ }_{73}^{157} \mathrm{Ta}$ & $20 \mathrm{nb}$ & $927(7)$ & & $12.1_{-2.3}^{+3.1} \mathrm{~ms}$ & $\mathrm{l}=0$ & $\begin{array}{l}\text { Page et al. } \\
\text { Irvine } \text { et al. } \\
(1996)(\mathrm{E}) \\
(1997)(\mathrm{E})\end{array}$ \\
\hline${ }_{75}^{159} \mathrm{Re}$ & & $1805(20)$ & & $21(4) \mu \mathrm{s}$ & $l=5$ & $\begin{array}{ll}\text { Joss et al. } & (2006) \\
\text { Page et al. } & (\mathrm{E}) \\
(2007) & (\mathrm{E}) \\
\end{array}$ \\
\hline${ }_{75}^{160} \mathrm{Re}$ & $1 \mu \mathrm{b}$ & $1261(6)$ & & $0.79(16) \mathrm{ms}$ & $l=2$ & 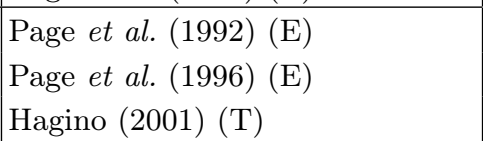 \\
\hline \begin{tabular}{|c}
${ }_{75}^{161} \mathrm{Re}$ \\
${ }_{75}^{161 m} \mathrm{Re}$
\end{tabular} & $150 \mathrm{nb}$ & $\begin{array}{l}1192(2) \\
1315(7)\end{array}$ & & $\begin{array}{c}440(1) \mu \mathrm{s} \\
14.7(3) \mathrm{ms}\end{array}$ & $\begin{array}{l}\mathrm{l}=0 \\
\mathrm{l}=5\end{array}$ & $\begin{array}{l}\text { Irvine et al. (1997) (E) } \\
\text { Lagergren et al. }(2006) \text { (E) } \\
\text { Hagino (2001) (T) } \\
\text { Arumugam et al. (2007) (T) }\end{array}$ \\
\hline
\end{tabular}

${ }^{a}$ see the discussion in (Page et al. 2007) about contradictory results from the work of (Uusitalo et al. 1999 ) 
TABLE V Summary of literature data for $s=1$ proton emitters. Continuation of Table III]

\begin{tabular}{|c|c|c|c|c|c|c|}
\hline Emitter & Cross sec. & $\mathrm{E}_{p}(\mathrm{MeV})$ & $\mathrm{Q}_{p}^{\text {nucl }}(\mathrm{MeV})$ & $\mathrm{T}_{1 / 2}$ & Ang.mom. & References \\
\hline \multirow[t]{2}{*}{${ }_{77}^{164 m} \operatorname{Ir}$} & \multirow[t]{2}{*}{$\mathrm{n} / \mathrm{a}$} & \multirow[t]{2}{*}{$1807(14)$} & & \multirow[t]{2}{*}{$58_{-18}^{+46} \mu \mathrm{s}$} & \multirow[t]{2}{*}{$\mathrm{l}=5$} & Kettunen et al. (2001) (E) \\
\hline & & & & & & Mahmud et al. (2002) (E) \\
\hline${ }_{77}^{165 m} \operatorname{Ir}$ & $0.2 \mu \mathrm{b}$ & $1707(7)$ & & $0.30(6) \mathrm{ms}$ & $l=5$ & Davids et al. (1997) (E) \\
\hline \multirow{2}{*}{\begin{tabular}{|c|}
${ }_{77}^{166} \mathrm{Ir}$ \\
${ }^{166 m} \mathrm{Ir}$
\end{tabular}} & \multirow[t]{2}{*}{$6 \mu \mathrm{b}$} & $1145(8)$ & & \multirow{2}{*}{$\begin{array}{c}10.5(2.2) \mathrm{ms} \\
15.1(9)\end{array}$} & \multirow{2}{*}{$\begin{array}{l}\mathrm{l}=2 \\
\mathrm{l}=5\end{array}$} & Davids et al. (1997) (E) \\
\hline & & $1316(8)$ & & & & \\
\hline \multirow{3}{*}{\begin{tabular}{|c|}
167 \\
${ }_{77} \mathrm{Ir}$ \\
$167 \mathrm{~m}$ \\
${ }_{77} \mathrm{Ir}$
\end{tabular}} & \multirow[t]{3}{*}{$10 \mu \mathrm{b}$} & \multirow{3}{*}{$\begin{array}{l}1064(6) \\
1238(7)\end{array}$} & & \multirow{3}{*}{$\begin{array}{c}35.2(20) \mathrm{ms} \\
30.0(6) \mathrm{ms}\end{array}$} & \multirow{3}{*}{$\begin{array}{l}\mathrm{l}=0 \\
\mathrm{l}=5\end{array}$} & Davids et al. (1997) (E) \\
\hline & & & & & & Davids and Esbensen (2000) (T) \\
\hline & & & & & & Scholey et al. (2005) (E) \\
\hline \multirow{2}{*}{\begin{tabular}{|c|}
${ }^{170} \mathrm{Au}$ \\
79 \\
$170 \mathrm{~m}$ \\
$79 \mathrm{Au}$
\end{tabular}} & & $1463(12)$ & & \multirow{2}{*}{$\begin{array}{c}286_{-40}^{+50} \mu \mathrm{s} \\
617_{-40}^{+50} \mu \mathrm{ms} \\
\end{array}$} & \multirow{2}{*}{$\begin{array}{l}\mathrm{l}=2 \\
\mathrm{l}=5\end{array}$} & Mahmud et al. (2002) (E) \\
\hline & & $1735(9)$ & & & & Kettunen et al. (2004) (E) \\
\hline \multirow{4}{*}{$\begin{array}{c}{ }_{79}^{171} \mathrm{Au} \\
171 m \\
{ }_{79} \mathrm{Au}\end{array}$} & \multirow[t]{4}{*}{$2 \mu \mathrm{b}$} & \multirow{4}{*}{$\begin{array}{c}1437(12) \\
1694(6)\end{array}$} & & \multirow{4}{*}{$\begin{array}{c}22_{-2}^{+3} \mu \mathrm{s} \\
1.09(3) \mathrm{ms}\end{array}$} & \multirow{4}{*}{$\begin{array}{l}\mathrm{l}=0 \\
\mathrm{l}=5\end{array}$} & Davids et al. (1997) (E) \\
\hline & & & & & & Poli et al. (1999) (E) \\
\hline & & & & & & Bäck et al. (2003) (E) \\
\hline & & & & & & Kettunen et al. (2004) (E) \\
\hline${ }_{81}^{176} \mathrm{Tl}$ & & $1258(18)$ & & $5.2_{-1.4}^{+3.0} \mathrm{~ms}$ & $l=0$ & Kettunen et al. (2004) (E) \\
\hline \multirow{3}{*}{$\begin{array}{c}177 \mathrm{Tl} \\
81 \mathrm{~m} \\
{ }_{81} \mathrm{Tl}\end{array}$} & \multirow[t]{3}{*}{$30 \mathrm{nb}$} & \multirow{3}{*}{$\begin{array}{l}1156(20) \\
1958(10)\end{array}$} & & \multirow{3}{*}{$\begin{array}{c}18(5) \mathrm{ms} \\
230(40) \mu \mathrm{s}\end{array}$} & \multirow{3}{*}{$\begin{array}{l}\mathrm{l}=0 \\
\mathrm{l}=5\end{array}$} & Poli et al. $(1999)(\mathrm{E})$ \\
\hline & & & & & & Davids et al. (2001) (E) \\
\hline & & & & & & Kettunen et al. (2004) (E) \\
\hline \multirow[t]{5}{*}{${ }_{83}^{185} \mathrm{Bi}$} & \multirow[t]{5}{*}{$60 \mathrm{nq}^{\mathrm{a}}$} & \multirow[t]{5}{*}{$1594(9)$} & & \multirow[t]{5}{*}{$60(4) \mu \mathrm{s}$} & \multirow[t]{5}{*}{$\mathrm{l}=0$} & Davids et al. (1996) (E) \\
\hline & & & & & & Poli et al. (2001) (E) \\
\hline & & & & & & Andreyev et al. 2004 (E) \\
\hline & & & & & & Andreyev et al. (2005) (E) \\
\hline & & & & & & Arumugam et al. (2007) (T) \\
\hline
\end{tabular}

a 6 -10nb for 3n evaporation channel (Andreyev:2004)

tions with tri-axially deformed potential are considered by Arumugam et al. (2009); Davids and Esbensen (2004); and Kruppa and Nazarewicz (2004). An extended description of the theoretical models used for proton emission can be found in chapters 4,5 , and 6 of the Lecture notes in Physics by Delion (2010), as well as in Delion et al. (2006b).

\section{B. Seniority $s \leq 2$ proton emitters}

Seniority $s \leq 2$ proton emitters are the "classical" proton emitters in which an unpaired proton leaves the nucleus from the ground state or the isomeric state. The isomeric state has to be low enough to allow only for unpaired particle excitation. The half-life of those emitters span from $T_{1 / 2}=1.9_{-0.5}^{+1.2} \mu$ s for ${ }^{144} \mathrm{Tm}$ to $0.560(40) \mathrm{s}$ for ${ }^{147} \mathrm{Tm}$. They were all but one produced in fusionevaporation reactions with the exit channel containing a proton and from 1 to 6 neutrons. ${ }^{185} \mathrm{Bi}$ was also produced in the $3 \mathrm{n}$ evaporation channel (Andreyev et al. 2004). Typical cross sections range from $0.3 \mathrm{nb}(1 \mathrm{p}, 6 \mathrm{n})$ for ${ }^{121} \operatorname{Pr}$ to $70 \mu \mathrm{b}(1 \mathrm{p}, 2 \mathrm{n})$ for ${ }^{151} \mathrm{Lu}$. Combined properties of "classical" proton emitters are presented in table III.

\section{Odd-mass, $s=1$ proton emitters}

As mentioned above, due to the strong dependence of the half-life on the $Q_{p}$-value and on the angular momentum of the emitted proton (see figure 23), proton emission is a valuable tool for nuclear structure study beyond the proton drip line. Measured proton energy and decay half-life in most of the cases directly point to the configuration of the decaying orbital. This is especially true for the odd-mass seniority $s=1$ cases where the emitting nuclei can be described as even-even $0^{+}$core coupled to the unpaired proton. With the $0^{+}$ground state of the daughter nuclei, establishing the angular momentum leaves only two possibilities for the total angular momentum $j=l \pm 1 / 2$ of the emitting state. This value can then be used to calculate the components of the nuclear wave function of the emitter. Depending on the nuclear shape either spherical single-particle orbitals or Nilsson type deformed orbitals can be used. Evolution of proton emitting states starts with ${ }^{109} \mathrm{I}(Z=53, l=2)$ proton radioactivity. In this region just above $Z=50$ the $\pi d_{5 / 2}$ and $\pi g_{7 / 2}$ orbitals are close to each other, nevertheless due to the lower $l$ of the former configuration $\left(\pi d_{5 / 2}\right)$ most of the emitted protons carry away two units of angular momentum. There is though one case discussed in the 
literature namely ${ }^{121} \mathrm{Pr}$, for which Robinson et al. (2005) suggests two possible configurations $3 / 2^{+}[422]$ from the spherical $\pi g_{7 / 2}$ orbital and $3 / 2^{-}[541]$ from the spherical $\pi h_{11 / 2}$ orbital. While in case of a high deformation the $3 / 2^{+}[422]$ state can contain admixtures of the spherical $\pi d_{3 / 2}$ and $\pi d_{5 / 2}$ orbitals, making the $l=2$ proton transition possible, the negative parity $3 / 2^{-}$[541] state cannot contain an $l=2$ contribution. In the Delion et al. (2006b) systematics $l=2$ is assigned to the proton emission from ${ }^{121} \mathrm{Pr}$ making it "compatible" with other proton emitters in this region.

Although for proton emitters above $Z=64$ three orbitals $\left(d_{3 / 2}, h_{11 / 2}\right.$ and $\left.s_{1 / 2}\right)$ should be considered as proton emitters, an interesting phenomenon occurs for ${ }^{135} \mathrm{~Tb}$ $(Z=65)$ and for ${ }^{141}$ Ho $(Z=67)$ due to the high deformation. The $7 / 2^{-}$ground states of both emitters are dominated by the [523] component from the $h_{11 / 2}$ orbital, but the proton emission is driven by a small admixture of the $f_{7 / 2}$ orbital to the wave function. The small $l=3$ component $\left(<2 \%\right.$ in case of $\left.{ }^{141} \mathrm{Ho}\right)$ wins over the larger $l=5$ component ( $>78 \%$ in case of ${ }^{141} \mathrm{Ho}$ ) (Karny et al. 2008). The presence of the $1 \pi f_{7 / 2}$ component in the wave function of nuclei in this region is also confirmed by the analysis of ${ }^{145} \mathrm{Tm}$ fine structure data. The $9.6 \%$ proton branching to the first excited $2^{+}$level in ${ }^{144} \mathrm{Er}$ can only be explained by the presence of the $l=3$ component in the wave function (Karny et al., 2003). The small energy difference between $h_{11 / 2}$ and $d_{3 / 2}$ related levels manifests itself through the presence of $l=2$ proton emitting low lying isomers in ${ }^{147 m} \mathrm{Tm}$ and ${ }^{151 m} \mathrm{Lu}$.

Odd-mass proton emitters above $Z=72$ are characterized by the presence of two proton emitting states $1 / 2^{+}$ground state and the $11 / 2^{-}$isomer. There are two cases ${ }^{159} \mathrm{Re}$ (Joss et al., 2006$)$ and ${ }^{155} \mathrm{Ta}$ (Page et al. 2007) where only one $l=5$ proton emission has been observed. In the case of ${ }^{159} \mathrm{Re}$, the expected half-life for $l=0$ emission is below $1 \mu \mathrm{s}$, which was beyond the capability of applied experimental technique. The ${ }^{155} \mathrm{Ta}$ has been observed as a second generation decay after ${ }^{159} \mathrm{Re}$ ion implantation and its subsequent $\alpha$ decay to ${ }^{155} \mathrm{Ta}$. Although the expected half-life of $l=0$ proton emission from ${ }^{155} \mathrm{Ta}$ is long enough to be observed, the combination of the low production cross section for the $1 / 2^{+}$state with a small detection efficiency for the second generation decays, may explain the non-observation of the $l=0$ proton channel. Further studies have still to confirm that observed decays in both cases do originate from the $11 / 2^{-}$isomeric states. The heaviest known proton emitter ${ }^{185} \mathrm{Bi}(Z=83)$ decays with the $l=0$ proton emission from the $1 / 2^{+}[400]$ intruder state pushed by the deformation above the $Z=82$ shell.

It is worth noting that the $s=1{ }^{141}$ Ho proton emitter is among the most extensively studied and understood isotopes beyond the proton-drip line. We know proton emission from both ground and isomeric states in ${ }^{141}$ Ho to the $0^{+}$ground state as well as first excited $2^{+}$
TABLE VI Proton energies $E_{p}$ and branching ratios $I_{p}^{\text {exp }}$ measured for proton emission channels from ${ }^{146} \mathrm{Tm}$ together with the calculated values based on the particle-core vibration coupling model (Hagino, 2001, Tantawy et al., 2006). $\mathrm{E}_{f}$ denote excitation energy of the final state in ${ }^{145} \mathrm{Er}$. The spin and parity for states in ${ }^{145} \operatorname{Er}$ is $1 / 2^{+}, 3 / 2^{+}, 11 / 2^{-}$, and $13 / 2^{-}$for the excitation energy of $0,175,253$, and $484 \mathrm{keV}$, respectively. All energies are in $\mathrm{keV}$.

\begin{tabular}{|c|c|c|c|c|c|}
\hline$E_{p}$ & $I_{p}^{\exp }(\%)$ & Wave function composition & $I_{p}^{c a l}$ & $\Delta l$ & $\overline{\mathrm{E}_{f}}$ \\
\hline & & ground state & & & \\
\hline & & $I^{\pi}=5^{-}, T_{1 / 2}=68(5) \mathrm{ms}$ & & & \\
\hline $938(4)$ & $13.8(9)$ & $2 \% \pi s_{1 / 2} \otimes \nu h_{11 / 2} \otimes 0^{+}$ & $(15)^{a}$ & 0 & 253 \\
\hline $1016(4)$ & $18.3(11)$ & $4 \% \pi f_{7 / 2} \otimes \nu s_{1 / 2} \otimes 2^{+}$ & $15^{5}$ & 3 & 175 \\
\hline & & $41 \% \pi h_{11 / 2} \otimes \nu s_{1 / 2} \otimes 2^{+}$ & 0.003 & 5 & 175 \\
\hline 1191(1) & $68.1(19)$ & $53 \% \pi h_{11 / 2} \otimes \nu s_{1 / 2} \otimes 0^{+}$ & 70 & 5 & 0 \\
\hline & & isomeric state & & & \\
\hline & & $I^{\pi}=10^{+}, T_{1 / 2}=198(3) \mathrm{ms}$ & & & \\
\hline $889(8)$ & $1.0(4)$ & $2.5 \% \pi f_{7 / 2} \otimes \nu h_{11 / 2} \otimes 2^{+}$ & 1.2 & 3 & 484 \\
\hline & & $41 \% \pi h_{11 / 2} \otimes \nu h_{11 / 2} \otimes 2^{+}$ & 0.04 & 5 & 484 \\
\hline $1120(1)$ & $100(1)$ & $55 \% \pi h_{11 / 2} \otimes \nu h_{11 / 2} \otimes 0^{+}$ & 98.6 & 5 & 253 \\
\hline & & $0.1 \% \pi h_{9 / 2} \otimes \nu h_{11 / 2} \otimes 0^{+}$ & 0.2 & 5 & 253 \\
\hline & & $0.4 \% \pi(l>5) \otimes \nu h_{11 / 2}$ & & & \\
\hline
\end{tabular}

a Value based on the experimental intensity ratios not predicted by the particle-core vibration coupling model

state in ${ }^{140}$ Dy (Davids et al., 1998; Karny et al., 2008; Rykaczewski et al. [1999). In these decays three different angular momenta $(l=0,2$, and 3$)$ are involved. Proton emission was also used in a recoil decay tagging study of this isotope allowing observation and interpretation of rotational bands up to $I^{\pi}=35 / 2^{-}$(Seweryniak et al. 2001). Theoretical works focused on ${ }^{141}$ Ho include Arumugam et al. (2009); Barmore et al. (2000); Davids and Esbensen (2000, 2004); Esbensen and Davids (2001); Fiorin et al. (2003); Kadmensky and Sonzogni (2000); and Kruppa and Nazarewicz (2004)

\section{Even-mass, $s=2$ proton emitters}

There are 17 even-mass $s=2$ proton emitters known. The lightest known is ${ }_{55}^{112} \mathrm{Cs}_{57}$ and ${ }_{81}^{176} \mathrm{Tl}_{95}$ is the heaviest. In these odd-odd nuclides the wave function compositions results from the interaction of the unpaired proton and neutron. For nuclei with the neutron number $N<82$ the valence neutron has no significant influence on the proton emission, i.e. proton emission from the odd-odd emitters follows the pattern of the odd-even emitters of the same element. For example, ${ }^{150} \mathrm{Lu}$ has two states decaying via proton emission: the ground state which emits $l=5$ proton and an isomeric state emitting proton with $l=2$. The same pattern is found in ${ }^{151} \mathrm{Lu}$, where the ground- and isomeric states decay with $l=5$ and $l=$ 
TABLE VII Properties of $s>2$ proton emitters.

\begin{tabular}{|c|c|c|c|c|c|c|}
\hline Emitter & $\mathrm{E}^{*}(\mathrm{MeV})$ & $\mathrm{E}_{p}(\mathrm{MeV})$ & $\mathrm{T}_{1 / 2}$ & Configuration & Ang.mom. & References \\
\hline \multirow[t]{2}{*}{${ }^{53 m} \mathrm{Co}$} & \multirow[t]{2}{*}{$3.197(29)$} & \multirow[t]{2}{*}{$1.57(3)$} & \multirow[t]{2}{*}{$242(15) \mathrm{ms}$} & \multirow[t]{2}{*}[\pif_{7/2}^{-1}\otimes\nuf_{7/2}^{-2}]{$19 / 2^{-}$} & \multirow[t]{2}{*}{$\mathrm{l}=9$} & Jackson et al. (1970) \\
\hline & & & & & & Cerny et al. (1970) \\
\hline${ }^{54 m} \mathrm{Ni}$ & $6.457(1)$ & $1.28(5)$ & $152(4) \mathrm{ns}$ & {$\left[\pi\left(f_{7 / 2}^{-1} p_{3 / 2}\right) \otimes \nu f_{7 / 2}^{-2}\right] 10^{+}$} & $l=5$ & Rudolph et al. (2008) \\
\hline \multirow[t]{2}{*}{${ }^{94 m} \mathrm{Ag}$} & \multirow[t]{2}{*}{$5.780(30)$} & \multirow[t]{2}{*}{$0.79(3)^{\mathrm{a}}$} & \multirow[t]{2}{*}{$0.39(4) \mathrm{s}$} & \multirow[t]{2}{*}[\pig_{9/2}^{-3}\otimes\nug_{9/2}^{-3}]{$21^{+}$} & \multirow[t]{2}{*}{$\mathrm{l}=4$} & Mukha et al. (2005) \\
\hline & & & & & & Cerny et al. (2009) \\
\hline
\end{tabular}

${ }^{a}$ A second transition of $1.01 \mathrm{MeV}$ reported by Mukha et al. (2005) has not been confirmed by the work of Cerny et al. (2009) and therefore is not included in the table.

2 proton emission, respectively. The situation changes when neutrons start filling $\nu f_{7 / 2}$ orbital above $N=82$. In these cases an attractive interaction due to the tensor force between $\pi d_{3 / 2}$ and $\nu f_{7 / 2}$ orbitals pushes the former above the $\pi s_{1 / 2}$ orbital leading to $l=2$ proton emission from the ground state. In other words, the $s_{1 / 2}$ ground state in odd-mass emitters is replaced by the $d_{3 / 2}$ ground state in odd-odd emitters of the same element. The $l=2$ emission from the even-mass isotopes has been observed for ${ }_{73}^{156} \mathrm{Ta}_{83},{ }_{75}^{160} \mathrm{Re}_{85},{ }_{77}^{166} \mathrm{Ir}_{89},{ }_{79}^{170} \mathrm{Au}_{91}$. The ${ }_{81}^{176} \mathrm{Tl}_{95}$ emits an $l=0$ proton from its ground state just like its neighbor ${ }^{177} \mathrm{Tl}$.

The ${ }^{146} \mathrm{Tm}, s=2$, proton emitter is the richest proton emitter known. There are 5 proton transition known in this case (Tantawy et al. 2006$)$. Three transitions are coming from the ground state and two were assigned to its isomeric state. Table VI shows experimental results obtained for these transitions together with the calculated wave functions components, based on the work of Tantawy et al. (2006) and Hagino (2001).

The ground state emits protons with $l=0,3$ and 5 , while the emission from the isomeric state has mainly the $l=5$ component. It is worth noting that in the cited calculation $l=0$ emission is due to the $\pi s_{1 / 2} \otimes \nu h_{11 / 2} \otimes 0^{+}$ $2 \%$ component, which is the isospin symmetric to the dominant $(53 \%) \pi h_{11 / 2} \otimes \nu s_{1 / 2} \otimes 0^{+}$.

\section{Seniority $s>2$ proton emitters}

In this category there are 3 proton emitters to be mentioned: ${ }^{53 m} \mathrm{Co}$ (Jackson et al., 1970 ), ${ }^{54 m} \mathrm{Ni}$ Rudolph et al., 2008) and ${ }^{94 m} \mathrm{Ag}$ (Mukha et al. 2005). They are all high spin, high excitation isomers with a multi-particle configuration of the wave function. The ${ }^{53 m} \mathrm{Co}$ was the first proton emitter discovered. $\mathrm{T}_{1 / 2}=242(15) \mathrm{ms}$. Its wave function is best described by the $\left[\pi f_{7 / 2}^{-1} \otimes\right.$ $\left.\nu f_{7 / 2}^{-2}\right] 19 / 2^{-}$configuration. The transition goes to the $0^{+}$ground state of ${ }^{52} \mathrm{Fe}$, thus the proton carries 9 units of angular momentum (Jackson et al. 1970$)$. The ${ }^{54 m} \mathrm{Ni}$ is the first and so far the only proton emitter produced in fragmentation reaction. The angular momentum of the emitted proton equals 5 , although the $\pi h_{11 / 2}$ orbital is not present in the proposed configuration of the emitting state. Both cases can not be described by the core plus proton model used in case of $s \leq 2$ proton emitters. In the case of ${ }^{94 \mathrm{~m}} \mathrm{Ag} l=4$ emission is assumed to originate from $\pi g_{9 / 2}$ orbital. The $21^{+}$isomeric state is created by three proton holes on the $g_{9 / 2}$ orbital coupled to three neutron holes on the $g_{9 / 2}$ orbital. Table VII shows the combined information on these high spin proton emitters.

\section{Outlook}

The wealth of nuclear data established by proton radioactivity studies is impressive and indicates that this field of research is mature and the applied experimental techniques are well advanced. They appear, however, to be still not sufficient to address potential proton emitters with atomic numbers below 50 . The low production rates and short half-lives, expected for these nuclei, present a challenge to the experimentalists. Observation of proton radioactivity in nuclei with $Z<50$ and establishing their properties will be important for calculations of the astrophysical $r p$-process. Of special interest are nuclei around the waiting points, like ${ }^{68} \mathrm{Se}$ (see a recent article of Rogers et al. (2011)), and the region just below ${ }^{100} \mathrm{Sn}$, at the expected end of the $r p$-process path.

The prospects for experimental studies of proton emission in the region between $N=82$ and $Z=82$ were discussed recently by Page (2011). 


\section{ALPHA DECAYS}

Emission of $\alpha$ particles belongs to the oldest known (together with $\beta$-decay) types of radioactivity. Its first theoretical description by Gamow (1928) and independently by Gurney and Condon (1928) was one of the early triumphs of quantum mechanics applied for the first time to the atomic nucleus. In particular the empirical law of Geiger and Nutall (1912) could be successfully explained. Presently, the calculations of the $\alpha$-decay lifetimes are performed in analogy to proton radioactivity by using Eq. 21 (Gurvitz and Kalbermann, 1987) where the proton spectroscopic factor $S_{p}$ is replaced by the $\alpha$ preformation factor $S_{\alpha}$. The latter measures the probability that the $\alpha$ particle is formed inside the mother nucleus. Combining the shell model with the cluster model proved to be successful in calculating the absolute $\alpha$ decay width of ${ }^{212} \mathrm{Po}$ (Varga et al., 1992). The result, $\Gamma=1.45 \cdot 10^{-15} \mathrm{MeV}$, agrees very well with the experimental value of $1.5 \cdot 10^{-15} \mathrm{MeV}$. The large body of experimental and theoretical findings about $\alpha$ decay mode is covered extensively in a number of books and reviews (Delion, 2010, Rasmussen, 1966, Roeckl, 1996). For a compilation of even-even $\alpha$-decay data see Akovali (1998). An extended version of the Geiger-Nutall rule has been recently proposed by Qi et al. (2009a b). Since this paper is devoted to the decays at the limits of stability, here we focus mainly on the latest studies of $\alpha$ decay close to the proton drip-line.

One of the regions which attracts attention is located above ${ }^{100} \mathrm{Sn}$, where due to the proximity of $N=50$ and $Z=50$ shell closures, the energy available for $\alpha$ decay is large enough to overcome the Coulomb barrier. This results in an island of $\alpha$ radioactivity for $52 \leq Z \leq 56$ and the neutron number $N$ up to 60 . Apart from the energy factor one has to note that the nuclei in this region are among the heaviest with protons and neutrons occupying the same type of single-particle orbitals. For these nuclei the active single-particle orbitals are $g_{7 / 2}$ and $d_{5 / 2}$ which differ in excitation energy by only a few hundred $\mathrm{keV}$. In the case of protons and neutrons occupying the same orbitals, their spatial overlap is maximized leading to the maximal preformation factor. For this reason alpha decays ${ }^{104} \mathrm{Te} \rightarrow{ }^{100} \mathrm{Sn}$ and ${ }^{106} \mathrm{Te} \rightarrow{ }^{102} \mathrm{Sn}$ are expected to be the best examples of the superallowed alpha decay (Macfarlane and Siivola, 1965, Roeckl, 1995).

While the search for the superallowed ${ }^{104} \mathrm{Te} \rightarrow{ }^{100} \mathrm{Sn}$ decay is still an ongoing effort, the successful measurements of ${ }^{105} \mathrm{Te} \rightarrow{ }^{101} \mathrm{Sn}$ have been reported Liddick et al., 2006, Seweryniak et al., 2006). In the work of Seweryniak et al. (2006) the decay of ${ }^{105} \mathrm{Te}$ was measured directly. Ions of ${ }^{105} \mathrm{Te}$ were produced in the fusionevaporation reaction of a ${ }^{58} \mathrm{Ni}$ beam impinging on a ${ }^{50} \mathrm{Cr}$ target. The products were separated by means of the Fragment Mass Analyzer of Argonne National Laboratory (Davids et al. 1992$)$ and implanted into a DSSSD

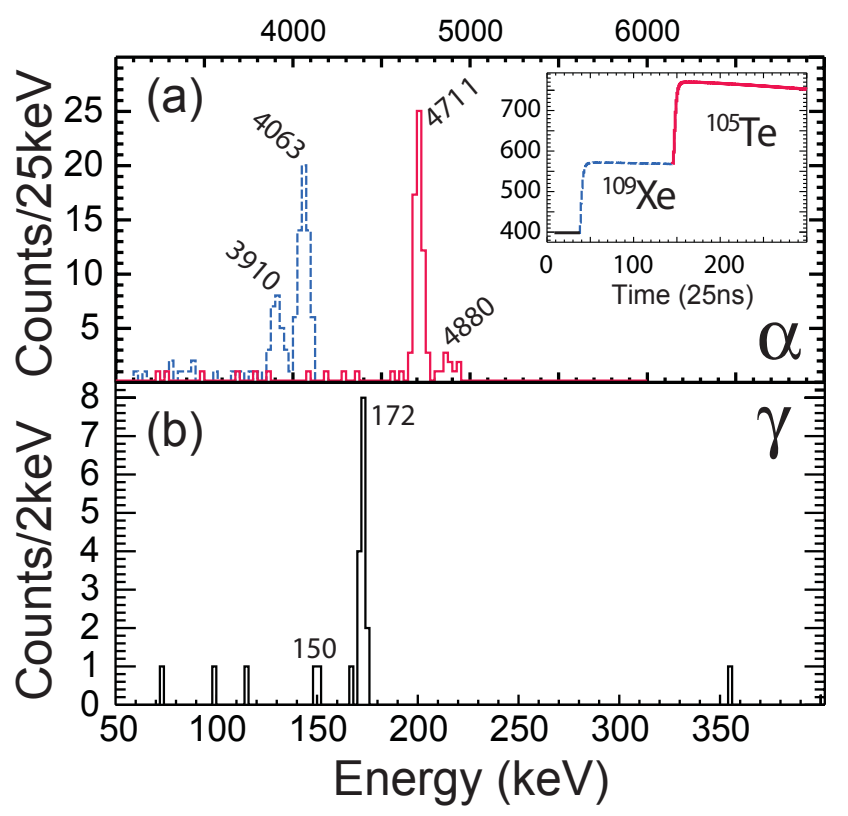

FIG. 24 (Color online) (a) Energy spectrum of the first $\left({ }^{109} \mathrm{Xe}\right)$ and the second $\left({ }^{105} \mathrm{Te}\right) \alpha$ pulses obtained from the $\alpha-\alpha$ pileup traces (inset). The lines at 3910(10) and 4063(4) $\mathrm{keV}$ are assigned to the ${ }^{109} \mathrm{Xe} \rightarrow{ }^{105} \mathrm{Te}$ transitions, while the lines at $4711(3)$ and $4880(20) \mathrm{keV}$ are assigned to the ${ }^{105} \mathrm{Xe} \rightarrow{ }^{101} \mathrm{Sn}$ decay. (b) $\gamma$ spectrum in coincidence with the analyzed $\alpha-\alpha$ traces. From (Darby et al. 2010).

detector. Alpha decays events were measured and correlated with the implanted ${ }^{105} \mathrm{Te}$ ions. Thirteen counts were identified as representing the decay ${ }^{105} \mathrm{Te} \rightarrow{ }^{101} \mathrm{Sn}$. As a result, an $\alpha$ decay energy $E_{\alpha}=4720(50) \mathrm{keV}$, corresponding to $\mathrm{Q}_{\alpha}=4900(50)$, and a half-life of $T_{1 / 2}=$ $0.7_{-0.17}^{+0.25} \mu$ s were established. In the different experiment of Liddick et al. (2006) the ${ }^{58} \mathrm{Ni}$ beam impinging on a ${ }^{54}$ Fe target was used. With the Recoil Mass Separator (RMS) of the HRIBF facility in Oak Ridge, the ${ }^{109} \mathrm{Xe}$ $\rightarrow{ }^{105} \mathrm{Te} \rightarrow{ }^{101} \mathrm{Sn}$ decay chain was analyzed. The relatively long half-life of ${ }^{109} \mathrm{Xe}(\sim 13 \mathrm{~ms})$ helped to overcome the inevitable losses due to the finite flight time through the RMS $(\sim 2 \mu \mathrm{s})$ in case ${ }^{105}$ Te was studied directly. Pulses of correlated $\alpha-\alpha$ decays were analyzed with help of digital electronics programmed to trigger and collect only the signals of high energy decay events. Analysis revealed two branches of ${ }^{109} \mathrm{Xe} \alpha$ decay with energies $E_{\alpha 1}=3918(9) \mathrm{keV}$ and $E_{\alpha 2}=4062(7) \mathrm{keV}$, followed by the $\alpha$ decay of ${ }^{105} \mathrm{Te}$ with the energy $E_{\alpha}=4703(5) \mathrm{keV}$. The half-lives of 13(2) ms and $0.62(7) \mu$ s were determined for ${ }^{109} \mathrm{Xe}$ and ${ }^{105} \mathrm{Te}$, respectively. The preformation factors derived from these experiments were found to be larger by about a factor of three from the values in the well studied region of the doubly magic ${ }^{208} \mathrm{~Pb}$ (Liddick et al. 2006, Mohr, 2007).

In the next experiment at the HRIBF laboratory, using a similar technique, the fine structure in the $\alpha$ decay of ${ }^{105} \mathrm{Te}$ was found (Darby et al. 2010 ). The setup used by 
Liddick et al. (2006) was additionally equipped with four germanium clover detectors placed around the DSSSD implantation detector. Double pulses of $\alpha$ - $\alpha$ events from the ${ }^{109} \mathrm{Xe} \rightarrow{ }^{105} \mathrm{Te} \rightarrow{ }^{101} \mathrm{Sn}$ decay chain were stored and analyzed. The result is shown in Fig. 24. The observed $\alpha$ lines at $4711 \mathrm{keV}$ and $4880 \mathrm{keV}$ are assigned to the decays of ${ }^{105} \mathrm{Te}$ leading to the first excited and to the ground state of ${ }^{101} \mathrm{Sn}$, respectively. The $\gamma$ transition between these two states $(172 \mathrm{keV})$, coincident with double $\alpha$ pulses was also detected (Fig. 24(b)). This result confirmed the previous evidence for the first excited state in ${ }^{101} \mathrm{Sn}$ obtained by Seweryniak et al. $(2007 \mathrm{~b})$ who employed the recoil-decay tagging method (RDT) (Paul et al. 1995) by combining $\gamma$ spectroscopy with $\beta$-delayed proton detection.

The stronger $\alpha$ line in the decay of ${ }^{105} \mathrm{Te}$, at $4711 \mathrm{keV}$ with the intensity of $89(4) \%$, is interpreted as corresponding to the decay with no change of the orbital angular momentum $(\Delta l=0)$, while the line at $4880 \mathrm{keV}$ is assigned to the $\Delta l=2$ channel (Darby et al., 2010). In addition, the strong $\Delta l=0$ transition goes to the first excited state in ${ }^{101} \mathrm{Sn}$ in contrast to the $\alpha$ decay of ${ }^{107} \mathrm{Te}$ where the strong $\Delta l=0$ decay connects nuclear ground states. Thus, the level inversion occurs between ${ }^{101} \mathrm{Sn}$ and ${ }^{103} \mathrm{Sn}$. The $5 / 2^{+}$ground state in ${ }^{103} \mathrm{Sn}$ becomes the first excited state in ${ }^{101} \mathrm{Sn}$, while the $7 / 2^{+}$excited state in ${ }^{103} \mathrm{Sn}$ becomes the ground state of ${ }^{101} \mathrm{Sn}$. This phenomenon is interpreted as a result of the interplay between the pairing on the $\nu g_{7 / 2}$ orbital $\left(V^{\text {pair }}\left(g_{7 / 2}\right)=1.4 \mathrm{MeV}\right)$ being much stronger than for the $\nu d_{5 / 2}$ orbital $\left(V^{\text {pair }}\left(d_{5 / 2}\right)=0.56 \mathrm{MeV}\right)$, and a small $(0.17 \mathrm{MeV})$ energy difference between these two orbitals (Darby et al. 2010 ). This interpretation contradicts the conclusions of Seweryniak et al. (2007b) who assigned spin and parity $5 / 2^{+}$to the ground state of ${ }^{101} \mathrm{Sn}$. We note that both experiments agree on their common experimental finding but differ in theoretical interpretation. Further experiments are required to firmly establish the $d_{5 / 2}-g_{7 / 2}$ order in ${ }^{101} \mathrm{Sn}$. For example, an observation (or exclusion) of the Gamow-Teller $\beta$-decay between the ground state of ${ }^{101} \mathrm{Sn}$ and the $9 / 2^{+}$ground state of ${ }^{101} \mathrm{In}$ should settle the controversy.

In the region of very neutron-deficient lead isotopes, recent $\alpha$ decay studies provided information on the shape coexistence in ${ }^{186} \mathrm{~Pb}$ (Andreyev et al., 2000). The states of ${ }^{186} \mathrm{~Pb}$ were populated in the $\alpha$ decay of ${ }^{190} \mathrm{Po}$, produced in the fusion-evaporation reaction of ${ }^{52} \mathrm{Cr}$ beam impinging on a ${ }^{142} \mathrm{NdF}_{3}$ target. The products were separated by means of the SHIP velocity filter (Münzenberg et al. 1979) at GSI Darmstadt and implanted into a position sensitive silicon detector, backed by a germanium clover detector for X-ray measurements. A set of silicon detectors was mounted for measurement of conversion electrons. In addition to the ground-state-toground-state decay, two other channels were observed in coincidence with conversion electrons. Due to the similar half-life all three alpha transitions were assigned to the decay of the ${ }^{190} \mathrm{Po}$ ground state. Analysis of coincidences between $\alpha$ particles, electrons, $\mathrm{X}$ - and $\gamma$-rays suggested that the spin of the three final states is $0^{+}$. The analysis of the preformation factor lead to the conclusion that the presence of the three $0^{+}$states in ${ }^{186} \mathrm{~Pb}$ similar energy, is a manifestation of shape coexistence where the ground state is spherical, the first excited $0^{+}$state at $532 \mathrm{keV}$ is oblate and second excited $0^{+}$state at $650 \mathrm{keV}$ is prolate.

It is worth noting that in recent years $\alpha$ decay served as a tagging signal in recoil decay tagging studies of heavy nuclei providing valuable nuclear structure information. Recent highlights from the RITU spectrometer at University of Jyväskylä were presented by Julin (2010). The RDT experiments with the FMA separator coupled to germanium detector arrays were reported by Carpenter et al. (1997), Reiter et al. (1999), and by Seweryniak et al. (1998, 2005b). Finally, it should be mentioned that most of the discoveries of new elements rely on $\alpha$ decay (Hofmann, 2009a). 


\section{TWO-PROTON RADIOACTIVITY}

\section{A. Introduction}

The two-proton radioactivity $(2 p)$ is the most recently observed type of decay and thus the least known. The experimental studies are still in the early stage. The detailed understanding of its mechanism requires novel theoretical approaches which in certain aspects are still under development. A very early look can be found in the book by Baz' et al. (1972). The current experimental and theoretical status of the $2 p$ decay were summarized recently by Blank and Płoszajczak (2008) and by Grigorenko (2009) with focus on specific theoretical methods. Because of its exceptional status, we discuss here this decay mode in more detail, emphasizing the major qualitative features of the phenomenon. The illustrations are provided mainly by the examples of ${ }^{6} \mathrm{Be},{ }^{19} \mathrm{Mg}$, and ${ }^{45} \mathrm{Fe}$. These nuclei belong to $p, s$ - $d$, and $p$ - $f$ shells respectively and their lifetimes span about 18 orders of the magnitude, providing support for universality of the currently achieved understanding of the two-proton decay.

The emission of two protons from a nuclear state is in principle possible in various decay scheme situations which are sketched in Fig. 25. We introduce here the following notation: $E_{T}$ is the system energy relative to the nearest three-body breakup threshold, while $E_{2 r}$ is the lowest two-body resonance energy relative to this threshold. The $2 p$ decay in the pure form, which we will call the true $2 p$ decay (or true three-body decay) is represented in Fig. 25(c). In this case sequential emission of protons is energetically prohibited and all final-state fragments are emitted simultaneously. Such a situation is common among even- $Z$ nuclei at the proton-drip-line and results from pairing interactions, see Sec.III. The decay dynamics of true $2 p$ decay is not reducible to the conventional twobody dynamics and should be addressed by the methods of few-body physics.

A somewhat special situation, represented in Fig. 25(d), occurs when the ground state of the subsystem is so broad that the emission of the first proton becomes energetically possible (although $E_{2 r}>E_{T}$ ) which opens a way for a sequential transition. Similarly, the decay may formally proceed in a sequential manner $\left(E_{2 r}<E_{T}\right)$ but the ground state of the subsystem is so broad that no strong correlation between outgoing fragments at given resonance energy can be formed, see Fig. 25(e). We refer to such scenarios as democratic decays and discuss them briefly in Sec. VII.B.1.

The three-body character of the $2 p$ radioactivity places it in the broader context of nuclear processes exhibiting essential many-body features. This includes studies of the broad states in continuum and excitation modes, like the soft dipole mode (Aumann, 2005). Another topic, pursued actively in the last decades, is the phenomenon of two-neutron halo (Zhukov et al. 1993) with its Bor- romean property that none of the three two-body subsystems is bound. The $2 p$ decay can be seen as an analogue of the two-neutron halo, requiring similar ingredients in the proper many-body description of its properties. The illustration of this point is provided by the isobaric mirror partners ${ }^{6} \mathrm{He}$ and ${ }^{6} \mathrm{Be}$ : the first is the "classical" Borromean halo nucleus and the second is the lightest true $2 p$ emitter. The crucial difference, however, comes from the fact that the $2 p$ decays involve charged particles in the continuum which significantly complicates the theoretical description. Another example: ${ }^{17} \mathrm{Ne}$ is a Borromean two-proton halo nucleus, while the first excited state of ${ }^{17} \mathrm{Ne}$ and the less bound ${ }^{16} \mathrm{Ne}$ are true $2 p$ emitters.

All ground-state two-proton emitters studied experimentally up to now are collected in Table VIII.

\section{Two-proton correlations}

The two-body decay of a resonance is characterized only by the energy and the width of the state. The threebody decay is much more "rich" as complex information about momentum correlations becomes available.

For decays with three particles in the final state there are nine degrees of freedom (spins are not counted). Three of them describe the center-of-mass motion and three describe the Euler rotation of the decay plane. Therefore, for a fixed decay energy $E_{T}$ there are two parameters representing the complete correlation picture. It is convenient to choose the energy distribution parameter $\varepsilon$ and an angle $\theta_{k}$ between the Jacobi momenta $\mathbf{k}_{x}$ and $\mathbf{k}_{y}$ :

$$
\begin{array}{r}
E_{T}=E_{x}+E_{y}=\frac{\left(A_{1}+A_{2}\right) k_{x}^{2}}{2 M A_{1} A_{2}}+\frac{\left(A_{1}+A_{2}+A_{3}\right) k_{y}^{2}}{2 M\left(A_{1}+A_{2}\right) A_{3}}, \\
\mathbf{k}_{x}=\frac{A_{2} \mathbf{k}_{1}-A_{1} \mathbf{k}_{2}}{A_{1}+A_{2}}, \quad \mathbf{k}_{y}=\frac{A_{3}\left(\mathbf{k}_{1}+\mathbf{k}_{2}\right)-\left(A_{1}+A_{2}\right) \mathbf{k}_{3}}{A_{1}+A_{2}+A_{3}}, \\
\varepsilon=E_{x} / E_{T}, \quad \cos \left(\theta_{k}\right)=\left(\mathbf{k}_{x} \cdot \mathbf{k}_{y}\right) /\left(k_{x} k_{y}\right),
\end{array}
$$

where $M$ is "scaling" nucleon mass, $M_{\text {nucleus }}=M\left(A_{1}+\right.$ $\left.A_{2}+A_{3}\right)$. The Jacobi momenta for two-proton emitters (protons are indistinguishable) can be defined in two "irreducible" Jacobi systems, called "T" and "Y", see Fig. 26 In the "T" Jacobi system, the core is the particle $A_{3}$ and the parameter $\varepsilon$ describes the energy distribution between the two protons. In the "Y" Jacobi system, the core is the particle $A_{2}$ and $\varepsilon$ corresponds to the core and proton subsystem. The Jacobi momentum $\mathbf{k}_{x}$ is the momentum of particle 1 in the c.m. of particles 1 and 2 , $\mathbf{k}_{y}$ is the c.m. momentum of particles 1 and 2 in the c.m. of the whole system (particles 1,2, and 3). Distributions constructed in the different Jacobi systems are just different representations of the same physical picture.

A more general (5-dimensional) correlation pattern becomes available for systems with total spin $J>1 / 2$. Manifestation of such correlations requires existence of 
(a)

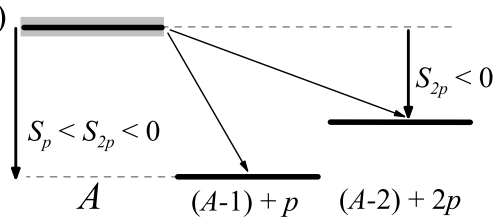

(b)

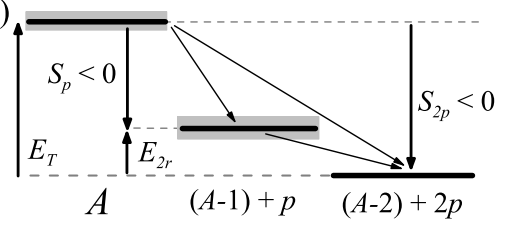

(c)

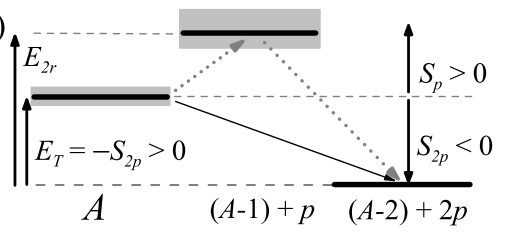

(d) $E_{2 r}$

(e)

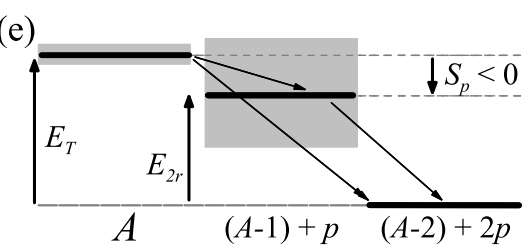

FIG. 25 Energy conditions for different modes of the two-proton emission: (a) typical situation for decays of excited states (both $1 p$ and $2 p$ decays are possible), (b) sequential decay via narrow intermediate resonance, (c) true $2 p$ decay. The cases (d) and (e) represent "democratic" decays. The gray dotted arrows in (c) and (d) indicate the "decay path" through the states available only as virtual excitations.

a selected direction in space and spin alignment, which is naturally achieved for short-lived states populated in nuclear reactions. The only example of such detailed studies is so far the two-neutron decay of broad states in ${ }^{5} \mathrm{H}$ (Golovkov et al. 2005)

\section{Historical note}

The possibility of a true two-proton emission was mentioned for the first time by Zeldovich (1960). This work comprises the dripline prediction for light systems. After predictions about existence of ${ }^{13} \mathrm{O}$ and ${ }^{20} \mathrm{Mg}$ isotopes Zeldovich notes: "The existence of ${ }^{12} \mathrm{O},{ }^{16} \mathrm{Ne}$, and ${ }^{19} \mathrm{Mg}$ is not excluded [...] These nuclei could appear to be unstable with respect to emission of two protons simultaneously." The explicit and detailed statement of the two-proton radioactivity phenomenon was given by V.I. Goldansky a bit later (Goldansky, 1960) 1. While the proton and cluster radioactivity are quite straightforward generalizations of $\alpha$-radioactivity, the few-body decays are qualitatively different and required ingenuity to foresee. The pioneering work of Goldansky contained several important insights which we illustrate by the following citation (Goldansky, 1960):

"Thus the simplest approach to the theory of twoproton decay would consist in using the product of two usual barrier factors, that is, in introducing an exponen-

\footnotetext{
${ }^{1}$ Zeldovich and Goldansky lived next door to each other. The problem is known to be a subject of many of their informal discussions (which are acknowledged in the paper of Goldansky). Later, on occasion of the priority discussion raised by some people, Zeldovich rejected any credits for the idea. Zeldovich was famous for providing in his works insights important for later development of physics in a very compact form and without attempt of further elaboration.
}

tial factor of the type

$$
w(\varepsilon)=\exp \left\{\frac{-2 \pi(Z-2) \alpha \sqrt{M}}{\sqrt{E_{T}}}\left[\frac{1}{\sqrt{\varepsilon}}+\frac{1}{\sqrt{1-\varepsilon}}\right]\right\},
$$

where $E_{T}$ is the sum of the energies of the two protons (energy of emitted diproton), $\varepsilon$ and $(1-\varepsilon)$ are the fractions of energy referring to each of the protons.

It can easily be seen that the total barrier factor $w(x)$ is maximum for $\varepsilon=0.5$, i.e., when the proton energies are equal. It will be noted that the value in the exponent is just the same as for the sub-barrier emission of a diproton with the energy $E_{T}$ as a whole.' ${ }^{2}$

The general character of the energy distribution predicted by Eq. (27) has proven to be correct and is now confirmed also experimentally. The idea of emission of a "diproton particle" turned to be an attractive concept but finally appeared to be misleading.

Later, significant theoretical work was devoted to identifying the best candidates for the observation of the $2 p$ radioactivity. Due to the extreme sensitivity of the $2 p$ decay probability to the width of the Coulomb barrier, the decay energy of a candidate must fall into a rather narrow window (Nazarewicz et al., 1996$)$. The resulting $2 p$ partial half-life should be long enough for an efficient separation in the spectrometer (typically a fraction of a microsecond) but short enough to compete with the $\beta^{+}$decay channel $(\sim 10 \mathrm{~ms})$. Thus accurate mass predictions for nuclei beyond the drip line were necessary. One of the most exact methods was the application of the isobaric multiplet mass equation (IMME) (Benenson and Kashy, 1979) combined with the experimentally measured mass of the neutron-rich member of the multiplet.

\footnotetext{
${ }^{2}$ In Eq. 27] we have modified the notation of Goldansky to make it consistent with the notation of this work.
} 
TABLE VIII Ground-state $2 p$ emitters investigated experimentally. The indicated half-life corresponds to the partial value for the $2 p$ decay.

\begin{tabular}{|c|c|c|c|}
\hline${ }^{N} Z$ & $E \mathrm{keV}$ & $\Gamma$ or $T_{1 / 2}$ & Reference \\
\hline${ }^{6} \mathrm{Be}$ & $1371(5)$ & $92(6) \mathrm{keV}$ & (Whaling, 1966) \\
\hline \multirow[t]{3}{*}{${ }^{12} \mathrm{O}$} & $1820(120)$ & $400(250)^{a} \mathrm{keV}$ & KeKelis et al. 1978) \\
\hline & $1790(40)$ & $580(200)^{\sqrt{a}} \mathrm{keV}$ & Kryger et al. 1995) \\
\hline & $1800(400)$ & $600(500)^{\sqrt{a}} \mathrm{keV}$ & (Suzuki et al. 2009) \\
\hline \multirow[t]{3}{*}{${ }^{16} \mathrm{Ne}$} & $1350(80)$ & $200(100)^{\sqrt{a}} \mathrm{keV}$ & (KeKelis et al. 1978) \\
\hline & $1400(20)$ & $110(40) \sqrt{a} \mathrm{keV}$ & (Woodward et al. 1983) \\
\hline & $1350(80)$ & $<200 \mathrm{keV}$ & (Mukha et al. 2008b) \\
\hline${ }^{19} \mathrm{Mg}$ & $750(50)$ & $4.0(15) \mathrm{ps}$ & (Mukha et al. 2007) \\
\hline \multirow[t]{4}{*}{${ }^{45} \mathrm{Fe}$} & $1100(100)$ & $3.2_{-1.0}^{+2.6} \mathrm{~ms}$ & (Pfützner et al. 2002) \\
\hline & $1140(50)$ & $5.7_{-1.4}^{+2.7} \mathrm{~ms}$ & (Giovinazzo et al., 2002) \\
\hline & $1154(16)$ & $2.8_{-0.7}^{+1.0} \mathrm{~ms}$ & (Dossat et al. 2005) \\
\hline & & $3.7_{-0.4}^{+0.4} \mathrm{~ms}$ & Miernik et al. 2007c) \\
\hline \multirow[t]{2}{*}{${ }^{48} \mathrm{Ni}$} & $1350(20)$ & $8.4_{-7.0}^{+12.8} \mathrm{~ms}$ & (Dossat et al. 2005$)$ \\
\hline & & $3.0_{-1.2}^{+2.2} \mathrm{~ms}$ & (Pomorski et al. 2011b) \\
\hline${ }^{54} \mathrm{Zn}$ & $1480(20)$ & $3.2_{-7.8}^{+1.8} \mathrm{~ms}$ & (Blank et al. 2005$)$ \\
\hline
\end{tabular}

a According to theoretical calculations much smaller widths are expected (Barker. 19992001 Grigorenko et al. 2002).

b Only one decay event observed.

Coefficients of the IMME can be calculated within the shell model or deduced from the Coulomb-energy systematics. Both approaches were undertaken (Brown, 1991. Cole, 1996: Ormand, 1996) and the choice of the best candidates was narrowed down to three cases: ${ }^{45} \mathrm{Fe},{ }^{48} \mathrm{Ni}$, and ${ }^{54} \mathrm{Zn}$. These predictions played an essential role in motivating experimental search for the $2 p$ radioactivity.

\section{B. Experimental results}

While the discovery of the true $2 p$ radioactivity awaited more than 40 years from its prediction, interesting information about related phenomena has been accumulated. In particular, this include $2 p$ democratic decays and the $2 p$ emission from excited nuclear states populated in $\beta$ decay and in reactions. In the following we overview the main steps in the experimental progress.

\section{Democratic decays}

Since it is relatively easier to reach experimentally light proton-drip line nuclei, the first attempts to search for $2 p$ emission phenomena started with the light $2 p$ unbound systems: ${ }^{6} \mathrm{Be}$ (Bochkarev et al., 1984, Geesaman et al. 1977), ${ }^{12} \mathrm{O}$ (KeKelis et al., 1978 ; Kryger et al. , 1995), and ${ }^{16} \mathrm{Ne}$ (KeKelis et al., 1978), see also Table VIII Due to a very low Coulomb barrier these cases have half-lives much too short to classify as being radioactive, more appropriately they should be referred to as $2 p$ resonances.

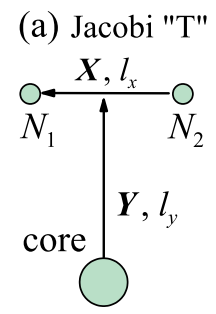

(b) Jacobi "Y"

(c) Shell model "V"

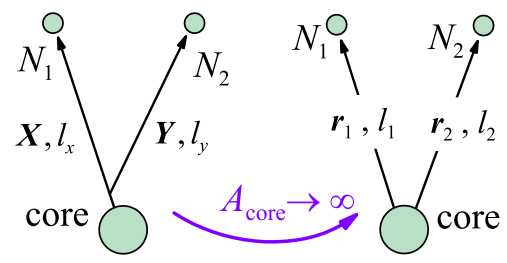

FIG. 26 (Color online) Coordinate systems for two-nucleon plus core problem. In the Jacobi "T" system (a), the "diproton" and the core are explicitly in configurations with definite angular momenta $l_{x}$ and $l_{y}$. For a heavy core the Jacobi "Y" system (b) is close to the single-particle "V"-system (c) used typically in many-body approaches.

Already Geesaman et al. (1977) could not fit the results on ${ }^{6}$ Be using simple decay scenarios (phase volume, diproton decay, simultaneous emission of $p$-wave protons) and concluded that a full three-body computation is necessary to understand the measured spectra. Later the interpretation of correlations observed in the decay of the ${ }^{6} \mathrm{Be}$ ground state have led to the concept of "democratic decay" (Bochkarev et al., 1989). In such a decay there is no strong energy focusing of the particles and they are smoothly distributed in the momentum space. The system is demonstrating a kind of equal rights among different parts of the kinematical space 3 . The democratic decay is not a phenomenon on its own but rather a name for the experimental fingerprint of a true three-body decay in light systems with the relatively small Coulomb force or in two-neutron emitters.

The study of opening angle between protons emitted from ${ }^{12} \mathrm{O}$ was motivated by the search for diproton correlation. The measured spectrum, however, was found to be consistent with the sequential emission via intermediate ${ }^{11} \mathrm{~N}$ state. Later it was found that indeed, the ground state energy of ${ }^{11} \mathrm{~N}$ is below that of ${ }^{12} \mathrm{O}$ and the decay of the latter belongs to the class shown in Fig. 25.e) (Azhari et al. . 1998).

Recently, the full correlation picture for protons emitted by ${ }^{6}$ Be has been experimentally established (Grigorenko et al. 2009b) and was found to be in very good agreement with the predictions of the three-body model which will be discussed below. Both experimental and theoretical distributions in the "T" and "Y" Jacobi coordinates are presented in Fig. 27. Similarly, the new results of $p$ - $p$ correlations in the decay of ${ }^{16} \mathrm{Ne}$ could be well described by the three-body model (Mukha et al.

\footnotetext{
${ }^{3}$ Now the term democratic decay has become accepted for description of the mentioned class of phenomena. There exist, however, an anecdotic story that when it was used for the first time at some conference at Soviet Union in the end of 80-s the authors were heavily criticized because "there can not be any democracy in nuclear physics".
} 


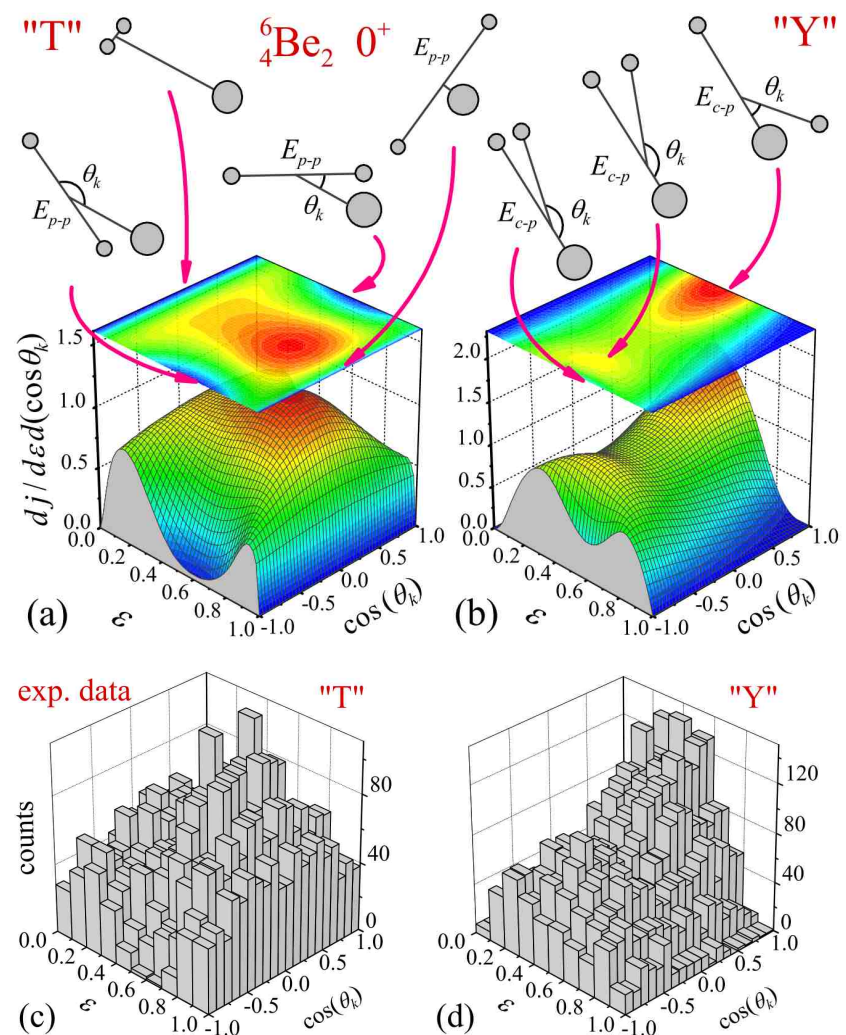

FIG. 27 (Colour online) Complete correlation picture for ${ }^{6} \mathrm{Be}$ g.s. decay, presented in "T" $(\mathrm{a}, \mathrm{c})$ and "Y" (b,d) Jacobi systems. The $(\mathrm{a}, \mathrm{b})$ is theory, $(\mathrm{c}, \mathrm{d})$ is experimental data. Qualitative illustration of the meaning of different kinematical regions is provided above the panels. Data and calculations are from (Grigorenko et al., 2009b).

2008b).

\section{Two-proton emission from excited states}

Several cases of $\beta$-delayed $2 p$ emission are known, as mentioned in Sec.IV.D They are discussed in more detail in Blank and Borge (2008). It is believed that in all cases studied, the mechanism of the emission is sequential, i.e. it can be described as a sequence of two two-body decays.

In a few other cases, the $2 p$ emitting excited states were populated by nuclear reactions. Like in the case of ground-state resonances, the main motivation of these studies was the search for $p-p$ correlations going beyond the sequential mechanism. The $2^{+}$state at $7.77 \mathrm{MeV}$ in ${ }^{14} \mathrm{O}$ was excited by the two-proton transfer reaction from ${ }^{3} \mathrm{He}$ impinging on a ${ }^{12} \mathrm{C}$ target (Bain et al., 1996). The $2 p$ emission from this state was found to proceed sequentially through a $1 / 2^{+}$state in ${ }^{13} \mathrm{~N}$. The first excited state in ${ }^{17} \mathrm{Ne}\left(3 / 2^{-}\right)$was reached by the Coulomb excitation of the radioactive ${ }^{17} \mathrm{Ne}$ beam to search for its $2 p$ decay (Chromik et al., 2002). Although the conditions for the true $2 p$ decay were fulfilled in this case, the de-excitation by $\gamma$ radiation was found to dominate. In a different approach, however, higher excited states in ${ }^{17} \mathrm{Ne}$ were populated by $1 \mathrm{n}$ stripping reaction from the radioactive ${ }^{18} \mathrm{Ne}$ beam (Zerguerras et al. 2004). The $2 p$ angular correlation was found to be peaked at small angles indicating a contribution from a seemingly non-sequential mechanism. Due to small statistics and limited information on the identity of the decaying states, however, no definite conclusions could be reached. The $1^{-}$resonance at $6.15 \mathrm{MeV}$ in ${ }^{18} \mathrm{Ne}$ was also thought to provide opportunity for the true $2 p$ emission, as no states are known in ${ }^{17} \mathrm{~F}$ to be located within the decay energy window. The $2 p$ emission from this state, populated in the ${ }^{17} \mathrm{~F}+{ }^{1} \mathrm{H}$ reaction, was measured and a diproton-like contribution to the $p$ - $p$ correlation spectra was claimed (del Campo et al. 2001). Similar evidence was announced in another work, in which the same state was populated by the Coulomb excitation of the ${ }^{18} \mathrm{Ne}$ beam (Raciti et al. 2008). The statistical significance of both claims, however, is weak. The excited states of ${ }^{19} \mathrm{Ne}$, populated in the inelastic scattering of ${ }^{18} \mathrm{Ne}$ on hydrogen target, were found to emit two protons sequentially (F. de Oliveira Santos et al. 2005).

An interesting case is the claimed $2 p$ emission from the high-spin $21^{+}$isomeric state in ${ }^{94} \mathrm{Ag}$ (Mukha et al. 2006). The authors explain an anomalously high $2 p$ decay rate and observed $p-p$ correlations by assuming a very high deformation of the isomer. This work has caused a lot of controversies (Cerny et al., 2009, Kankainen et al. 2008 , Mukha et al. 2008a; Pechenaya et al. | 2007) which call for further experimental investigations.

\section{Two-proton radioactivity}

The first case of $2 p$ radioactivity was found in 2002 in the decay of ${ }^{45} \mathrm{Fe}$ measured at GSI Darmstadt (Pfützner et al. 2002) and at GANIL (Giovinazzo et al., 2002). In both experiments ions of ${ }^{45} \mathrm{Fe}$ were produced by the fragmentation reaction of a ${ }^{58} \mathrm{Ni}$ beam and separated using the in-flight technique (Sec. III.B.1). The selected ions were implanted into silicon detectors and the only observables measured were the decay time and energy. It sufficed to claim the observation of a new decay mode because only the $2 p$ emission hypothesis was consistent with the measured data. Later, in another GANIL experiment the information on ${ }^{45} \mathrm{Fe}$ was obtained with larger statistics (30 atoms) and improved accuracy (Dossat et al. 2005), see Table VIII and Fig. 28. With the same experimental technique, applied again at GANIL laboratory, ${ }^{54} \mathrm{Zn}$ has been identified to decay by the $2 p$ radioactivity (Blank et al. 2005). One decay event of ${ }^{48} \mathrm{Ni}$ was found to coincide with the $2 p$ decay energy predicted for this nucleus (Dossat et al. 2005).

In the next step, gaseous detectors, based on the principle of the time projection chamber (TPC), were devel- 

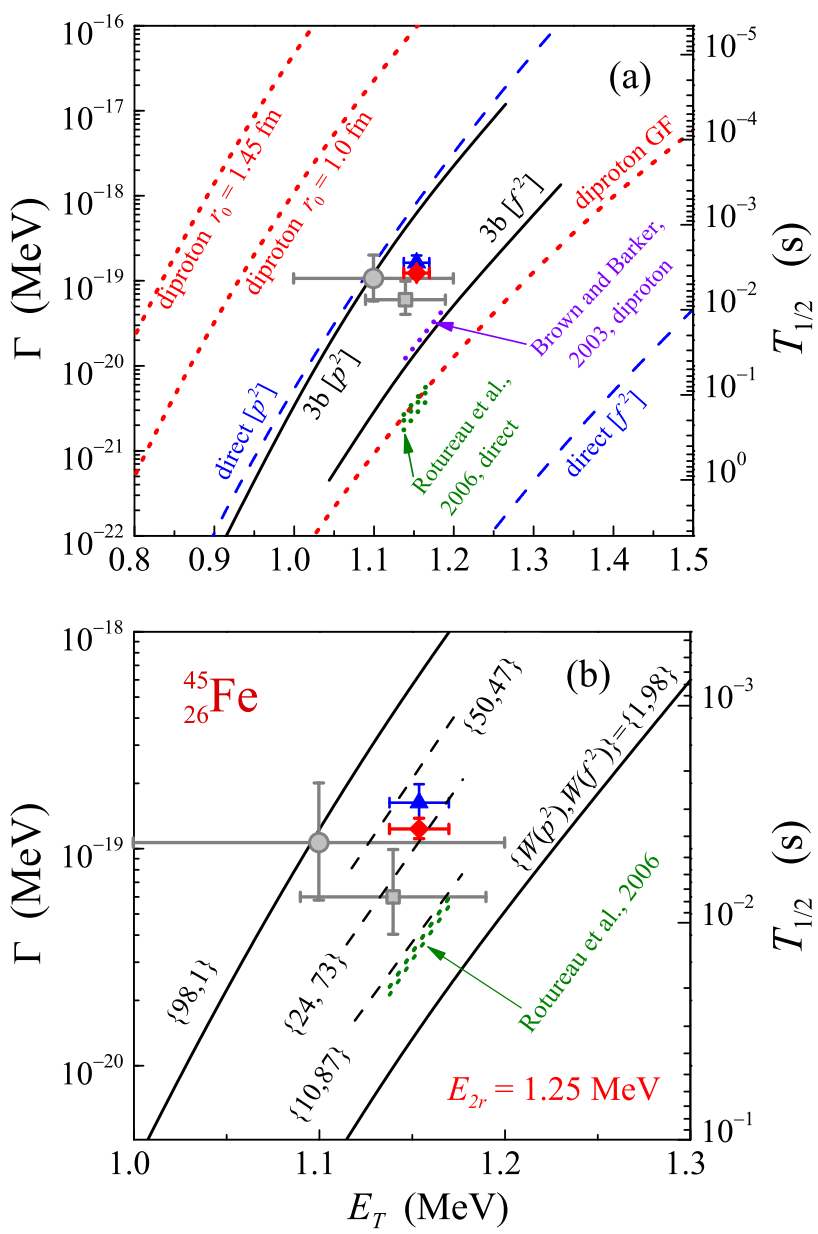

FIG. 28 (Color online) Lifetime of ${ }^{45} \mathrm{Fe}$ vs. decay energy calculated in different models. (a) Simplified models of $2 p$ decay. All spectroscopic factors are taken as unity. (b) Three-body model (Grigorenko et al. 2003a, Grigorenko and Zhukov, 2007a) and continuum shell model results (Rotureau et al. 2006). The experimental points demonstrate the rapid improvement of the data: circle (Pfützner et al., 2002), square (Giovinazzo et al., 2002), triangle (Dossat et al. 2005), and diamond (Miernik et al. 2007c).

oped to directly record emitted protons and to establish the correlations between them. The first direct observation of the two protons ejected from ${ }^{45} \mathrm{Fe}$ was achieved by Giovinazzo et al. (2007) who recorded projections of protons' tracks on the anode plane of the TPC. Later, this detector was used to directly demonstrate the twoprotons emitted in the decay of ${ }^{54} \mathrm{Zn}$ (Blank et al. 2011$)$. Miernik et al. (2007c) applied a novel type of detector, utilizing the optical readout of the TPC signals (OTPC, see Sec. III.C.1), to the detailed decay study of ${ }^{45} \mathrm{Fe}$ at the NSCL/MSU laboratory and succeeded to fully reconstruct tracks of emitted protons in three dimensions. The full correlation picture for the $2 p$ decay of ${ }^{45} \mathrm{Fe}$ established in this experiment is shown in Fig. 29. Recently, the OTPC detector was used to the decay study of ${ }^{48} \mathrm{Ni}$ at the NSCL/MSU laboratory and provided a direct and unambiguous evidence for the $2 p$ radioactivity of this nuclide (Pomorski et al. 2011b). From the six decays recorded, four corresponded to the $2 p$ emission and two were interpreted as $\beta$-delayed proton emission. An example of a $2 p$ decay event of ${ }^{48} \mathrm{Ni}$ is shown in Fig. 30.

An application of the different technique based on decay in-flight and particle tracking (Sec. III.C.1) is necessary to extend decay studies to very short half-lives in the subnanosecond range. This technique was used by Mukha et al. (2007) to investigate the $2 p$ radioactivity of ${ }^{19} \mathrm{Mg}$ whose half-life was found to be $4.0(15) \mathrm{ps}$. The $p-p$ momentum distributions, projected on the transverse detector plane were obtained in (Mukha et al. 2008b), see Fig. 31 .

\section{Simplified theoretical models}

Simplified theoretical models of three-body decays are typically based on the factorization of the decay amplitude into the product of two-body terms. For $2 p$ emission this factorization can be done either in the "T" or in the "Y" Jacobi systems, see Fig. 26. This results in formulation of diproton model or the direct decay model respectively. Factorization of amplitudes becomes possible only for specific forms of the underlying Hamiltonians (Grigorenko and Zhukov, 2007a). Then, neglecting some of the final state interactions (FSI), the three-body Green's function (GF) can be constructed in an analytical form:

$$
\begin{array}{r}
\hat{G}_{3 E}^{(+)}\left(\mathbf{X Y}, \mathbf{X}^{\prime} \mathbf{Y}^{\prime}\right)=\frac{E}{2 \pi i} \int_{0}^{1} d \varepsilon \hat{G}_{\varepsilon E}^{(+)}\left(\mathbf{X}, \mathbf{X}^{\prime}\right) \\
\times \hat{G}_{(1-\varepsilon) E}^{(+)}\left(\mathbf{Y}, \mathbf{Y}^{\prime}\right) .
\end{array}
$$

The operator $\hat{G}_{E}^{(+)}$is the ordinary two-body GF for the respective subsystem. This method allows to take into account one (out of three present) FSI exactly in diproton model. In the direct decay model two FSIs can be treated exactly but only in the limit of infinitely heavy core, see Figs. 26 (b,c). For heavy $2 p$ emitters this is a good approximation. The availability of approximate but analytic GF allows to determine decay properties without further approximations.

\section{Direct decay model}

The decay amplitude can be factorized in the "V" coordinate system [Fig. 26(c)] if we neglect $p$ - $p$ interaction and also assume an infinitely heavy core. The dominating contribution to the width can be obtained in the singlepole approximation for two-body GFs where only the lowest states in the subsystems core $+p$ with resonance energies $E_{p_{1}}$ and $E_{p_{2}}$ are considered (Galitsky and Cheltsov, 

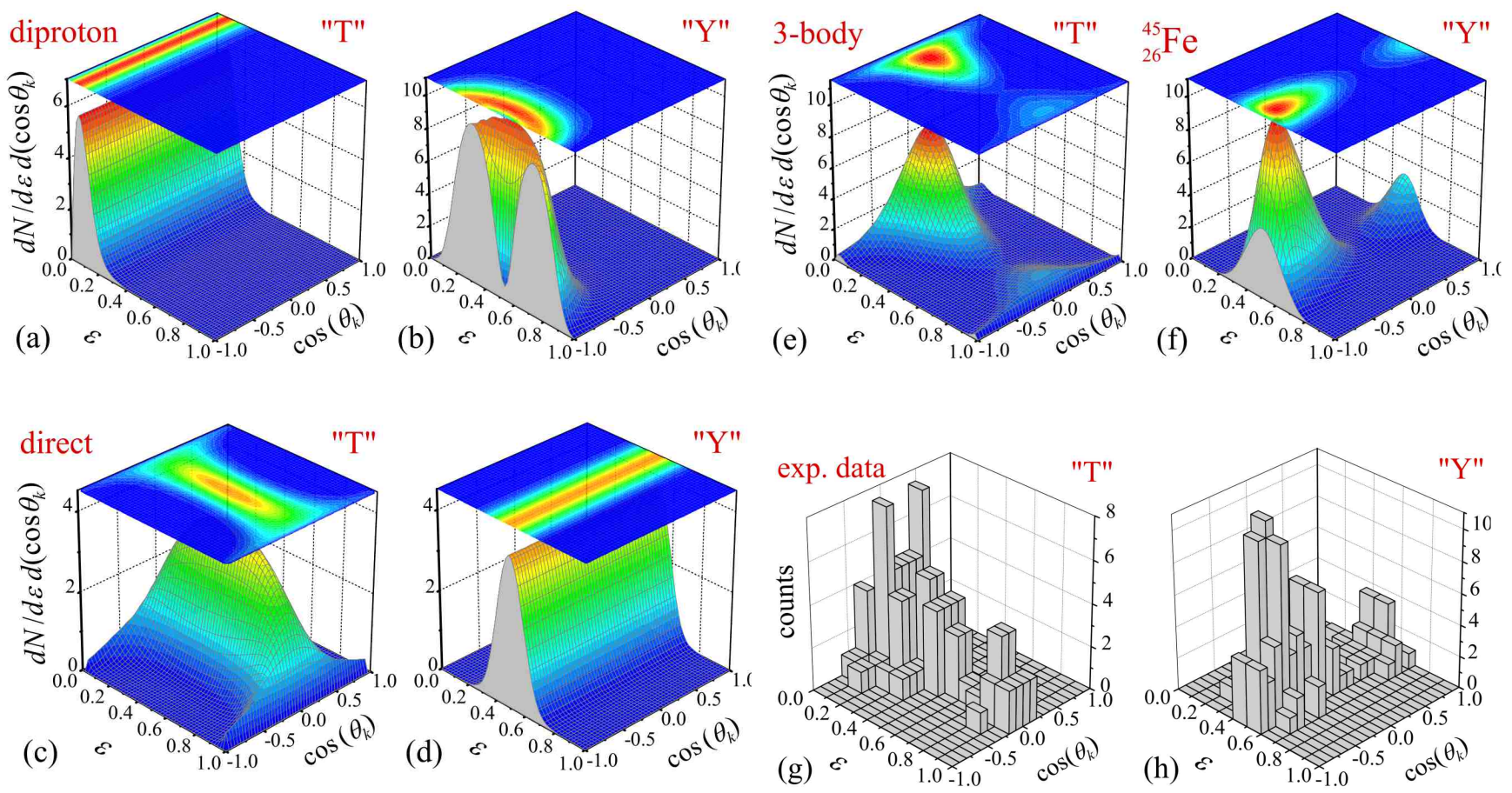

FIG. 29 (Color online) Momentum density distribution on the kinematical plane $\left\{\varepsilon, \cos \left(\theta_{k}\right)\right\}$ for ${ }^{45}$ Fe. Diproton model (a,b), direct decay model $(\mathrm{c}, \mathrm{d})$, three-body model $(\mathrm{e}, \mathrm{f})$, and experimental distribution $(\mathrm{g}, \mathrm{h})$. Correlation patterns are provided in the "T" (a,c,e,g) and "Y" (c,d,f,h) Jacobi systems. The calculations (e,f) are from (Grigorenko et al., 2010) and data (g,h) are from (Miernik et al., 2007c).

1964):

$$
\begin{aligned}
\Gamma_{\text {dir }}\left(E_{T}\right) & =\frac{E_{T}\left\langle V_{3}\right\rangle^{2}}{2 \pi} \int_{0}^{1} d \varepsilon \frac{\Gamma_{p_{1}}\left(\varepsilon E_{T}\right)}{\left(\varepsilon E_{T}-E_{p_{1}}\right)^{2}+\Gamma_{p_{1}}\left(\varepsilon E_{T}\right)^{2} / 4} \\
\times & \frac{\Gamma_{p_{2}}\left((1-\varepsilon) E_{T}\right)}{\left((1-\varepsilon) E_{T}-E_{p_{2}}\right)^{2}+\Gamma_{p_{2}}\left((1-\varepsilon) E_{T}\right)^{2} / 4},(29)
\end{aligned}
$$

It was shown in (Grigorenko and Zhukov, 2007b) that the matrix element $\left\langle V_{3}\right\rangle$ can be well approximated as

$$
\left\langle V_{3}\right\rangle^{2}=D_{3}\left(E_{T}-E_{p 1}-E_{p 2}\right)^{2} .
$$

The parameter $D_{3}$ is a constant, $D_{3} \approx 1.0-1.5$. The $\Gamma_{p_{i}}$ is the width of the ground state resonance in the core $+p$ subsystem. It can be expressed using the R-matrix approach to nuclear reactions (Lane and Thomas, 1958):

$$
\Gamma_{p_{i}}(E)=2 \gamma^{2} P_{l_{i}}\left(E, R, Z_{1} Z_{2}\right)
$$

The penetrability $P_{l}$ is defined via the Coulomb functions $F$ and $G$ (Abramowitz and Stegun, 1968), regular and irregular at the origin:

$$
P_{l}\left(E, R, Z_{1} Z_{2}\right)=\frac{k R}{F_{l}^{2}(\eta, k R)+G_{l}^{2}(\eta, k R)},
$$

In the above $\eta=Z_{1} Z_{2} \alpha M / k$ is the Sommerfeld parameter, $\alpha$ is the fine structure constant. The $\gamma^{2}$ is the reduced width which is factorized into the "Wigner limit" $\gamma_{W L}^{2}$ :

$$
\gamma^{2}=\gamma_{W L}^{2} \theta^{2}=\frac{1}{2 M R^{2}} \theta^{2},
$$

and the dimensionless reduced width $\theta^{2}$ (spectroscopic factor). The parameter $R$ is the "channel radius", typically taken as:

$$
R=r_{0}\left(A_{\text {core }}+1\right)^{1 / 3},
$$

where $r_{0}=1.4 \mathrm{fm}$.

The results of calculations in the direct decay approximation, Eq. 29), for the case of ${ }^{45} \mathrm{Fe}$ and for the values of angular momentum $l=1$ and $l=3$ are presented in Fig. 28 These are likely to provide upper and lower width limits for given decay energy which are consistent with the limits obtained in the complete three-body model, see Sec. VII.D.1.

Eq. 29) has a similar structure and can be considered as a refined version of the original expression (27) proposed by Goldansky. Compared to the latter it provides quantitative results (the preexponent is defined) and brings the explicit dependence on the property of the core $+p$ subsystem. The energy distribution between the core and one of the protons predicted by the direct decay approximation has a narrow bell-shape peaked at 


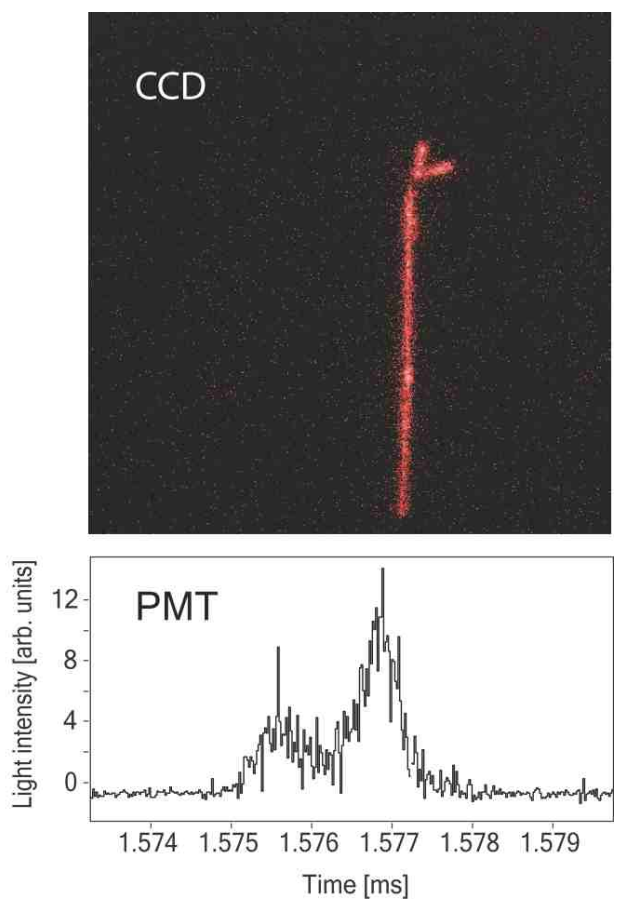

FIG. 30 An example of a two-proton decay event of ${ }^{48} \mathrm{Ni}$ recorded with the optical time projection chamber described in section III.C.1. Top: the image recorded by the CCD camera showing a long track of the ${ }^{48} \mathrm{Ni}$ ion entering the chamber from below and the two bright, short tracks of protons emitted $1.576 \mathrm{~ms}$ after the implantation. Bottom: a part of the time profile of the total light intensity measured by the PMT showing in detail the $2 p$ emission. From (Pomorski et al. 2011b).

$\varepsilon=1 / 2$, which reflects the symmetry between emitted protons, see Fig. 32. With increase of the atomic number this distribution becomes narrower. The bell-shape profile of this inclusive distributions is by now well confirmed by experiment (Blank et al., 2011; Grigorenko et al. 2009b; Miernik et al. | 2007c). However, looking at the complete distribution (see Fig. 29 that shows the distribution for the case of angular coupling $\left.\left[p_{1 / 2}^{2}\right]_{0}\right)$ the deficiency of the model becomes evident. Qualitatively, the direct decay model angular distribution always has backward-forward symmetry, while there is a strong angular asymmetry observed in experiment.

Using the fact that the numerator in Eq. 29) is sharply peaked at $\varepsilon / 2$ and the denominator for the true $2 p$ decays is a smooth function within the decay window, it can be further approximated:

$$
\begin{aligned}
\Gamma_{\mathrm{dir}}\left(E_{T}\right) & \approx \frac{E_{T} D_{3}\left(E_{T}-E_{p 1}-E_{p 2}\right)^{2}}{2 \pi\left(E_{T} / 2-E_{p 1}\right)^{2}\left(E_{T} / 2-E_{p 2}\right)^{2}} \\
& \times \int_{0}^{1} d \varepsilon \Gamma_{p_{1}}\left(\varepsilon E_{T}\right) \Gamma_{p_{2}}\left((1-\varepsilon) E_{T}\right),
\end{aligned}
$$

This approximation allows to estimate how the decay width depends on the position of the lowest resonances
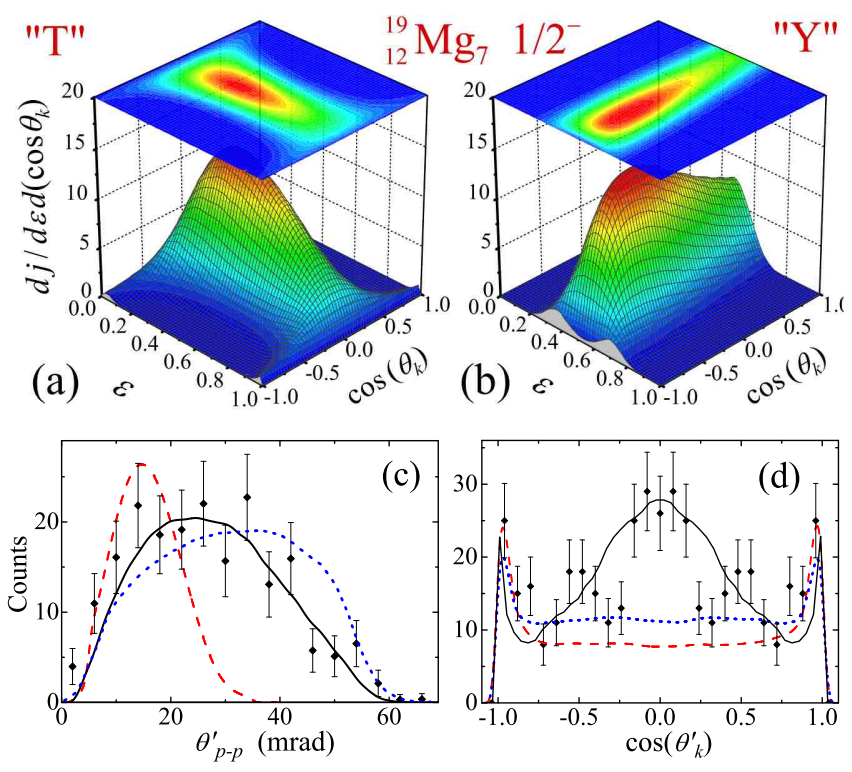

FIG. 31 (Colour online) Complete correlation picture for ${ }^{19} \mathrm{Mg}$ g.s. decay, presented in "T" (a) and "Y" (b) Jacobi systems. Comparison of two inclusive distributions projected on a plane is given in the lower row (Mukha et al., 2008b). The angles $\theta_{p-p}^{\prime}$ and $\theta_{k}^{\prime}$ are between projected momenta of protons (c) and projected Jacobi momenta in the "T" system (d). Solid, dashed, and doted curves correspond to threebody model, diproton model, and phase space simulations. Data are from (Mukha et al., 2008b) and calculations are from (Grigorenko et al. |2010).

in the core- $p$ subsystem. For the ground state true $2 p$ emitters $E_{p 1}=E_{p 1}=E_{2 r}$ and we get :

$$
\Gamma_{\text {dir }} \sim\left(E_{T} / 2-E_{2 r}\right)^{-2} .
$$

We note that a somewhat different expression for the width, in the analogous approximation, was introduced in Refs. (Azhari et al. 1998, Kryger et al. 1995) for ${ }^{12} \mathrm{O}$ :

$$
\Gamma_{\mathrm{kr}}=\frac{E_{T}}{2 \pi} \int_{0}^{1} d \varepsilon \frac{\Gamma_{p_{1}}\left(\varepsilon E_{T}\right) \Gamma_{p_{2}}\left((1-\varepsilon) E_{T}\right)}{\left(\varepsilon E_{T}-E_{p_{1}}\right)^{2}+\Gamma_{p_{1}}\left(\varepsilon E_{T}\right)^{2} / 4} .
$$

The expression is given here in our notation. It was introduced by analogy with two-body R-matrix expressions. Using the same approximation as in Eq. (35) we obtain

$$
C=\frac{\Gamma_{\mathrm{dir}}}{\Gamma_{\mathrm{kr}}} \approx D_{3} \frac{\left(E_{T}-E_{p_{1}}-E_{p_{2}}\right)^{2}\left(E_{T} / 2-E_{p_{1}}\right)^{2}}{\left(E_{T} / 2-E_{p_{1}}\right)^{2}\left(E_{T} / 2-E_{p_{2}}\right)^{2}} .
$$

For the ground state true $2 p$ emitters $C \approx 4$. In the series of papers (Barker, 1999, 2001, 2002, 2003, Brown and Barker, 2003| Brown et al. | 2002) formulae equivalent to Eq. (37) were used. The results obtained in these works should include the factor $C$ varying between 2 and 4 to be consistent with the direct decay approximation. 


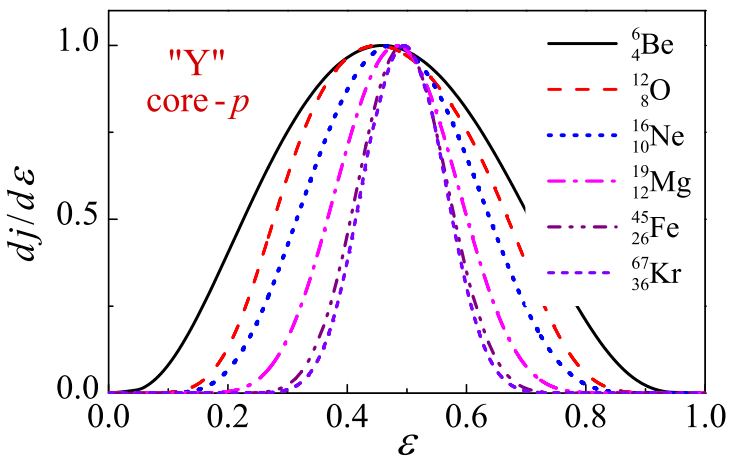

FIG. 32 (Color online) Systematics of energy distributions in the "Y" Jacobi system (core- $p$ channel) calculated in the direct decay model of Eq. 29). All distributions are normalized to unity at the maximum.

\section{Simultaneous vs. sequential decay}

The true two-proton emission process is sometimes interpreted as "sequential decay via tails of higher-lying resonances". Indeed, Eq. (36) shows that such resonances are very important for the true two-proton emission process. However, we shall argue that the sequential interpretation may be misleading. It can be shown that the resonance scattering can be interpreted in terms of the time delay $T_{l}(E, R)$ [e.g. (Baz', 1967)]:

$$
T_{l}(E, R) \sim \frac{\Gamma(E) / 4}{\left(E_{r}-E\right)^{2}+\Gamma(E)^{2} / 4} .
$$

Let us estimate the distance which the "first" emitted proton can travel while the "second" is "confined" in the tail of the resonance. Among the known and prospective two-proton emitters only the lightest ${ }^{6} \mathrm{Be}$ and ${ }^{12} \mathrm{O}$ have such flight path comparable or exceeding $\sim 1 \mathrm{fm}$. It can be seen in Fig. 33 (a) that for ${ }^{12} \mathrm{O}$ the estimated flight path can achieve $8 \mathrm{fm}$, but the contribution of such situations to the total decay probability is minor, Fig. 33 (b). Furthermore, having a typical scattering length in the nucleon-nucleon channel around $20 \mathrm{fm}$ even for ${ }^{12} \mathrm{O}$ we can not maintain that a reliable spatial separation of the core $+p$ subsystem is present.

So, the poles of the Greens' functions for two-body subsystems strongly increase the probability of true twoproton decay when they come close to the three-body decay window. However, only for one nuclear system $\left({ }^{12} \mathrm{O}\right)$ we can see considerable sequential separation of subsystems in space and even in that case the estimated contribution of sequential decay mechanism to the width is minor.

Important insight about the transition from true threebody to sequential decay can be obtained using the direct decay model. In Fig. 34 the results of calculations for ${ }^{6} \mathrm{Be},{ }^{12} \mathrm{O}$, and ${ }^{19} \mathrm{Mg}$ with help of Eq. 29 are shown for a fixed energy $E_{2 r}$ of the resonance in the core $+p$ subsystem as a function of the three-body energy $E_{T}$. We see
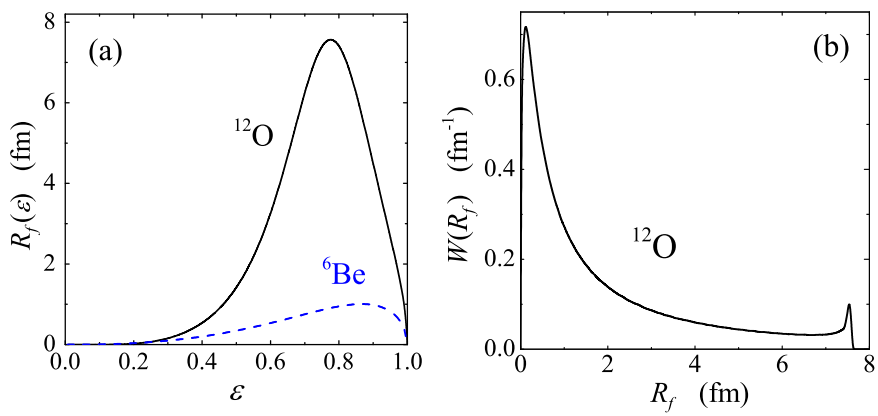

FIG. 33 (Color online) (a) Classical one-proton flight path $R_{f}$ for ${ }^{12} \mathrm{O}$ and ${ }^{6} \mathrm{Be}$ connected with the time delay in the core $+p$ subsystem calculated by Eq. (39). (b) Probability of different $R_{f}$ values for the decay of ${ }^{12} \mathrm{O}$ estimated by Eq. 29.

a difference between the democratic decays (broad states in the subsystems, overlapping with the decay window) and a radioactive decay (narrow state in the subsystem). The curve for ${ }^{19} \mathrm{Mg}$ in Fig. 34(a) shows two components: three-body regime (true three-body decay) and two-body regime (sequential decay) with a narrow transition zone in between (a kink in the line). In the cases of ${ }^{6} \mathrm{Be}$ and ${ }^{12} \mathrm{O}$ (democratic decays) there is no sharp difference visible between the two decay regimes and the transition is smooth.

The transition from the three-body to the two-body regime can be also illustrated by the energy distribution in the core- $p$ channel. Again, there is a qualitative difference between democratic and radioactive decays. In the former case, when the two-body resonance "enters" the three-body energy window, the width of the distribution suddenly becomes smaller, see Fig. 34(b). On the other hand, if the state in the subsystem is narrow, Fig. 34 (c), the two "horns" appear in the energy distribution corresponding to the proton energies defined by the intermediate state. As long as the difference $E_{T}-E_{2 r}$ is small, these horns do not contribute significantly to the total width and the decay remains effectively of the threebody type. Gradually the horns become dominating, while the relative contribution of the three-body "bell" at $\varepsilon=E_{T} / 2$ vanishes. It is reasonable to put the borderline between true three-body decay and sequential decay as the moment when the probabilities in the bell-like profile at $\varepsilon=E_{T} / 2$ and in the sequential horns become equal. From this one can derive a criterion for the three-body decay mechanism:

$$
\varepsilon_{0} E_{T}<E_{2 r}
$$

where for radioactive decays $\varepsilon_{0} \approx 0.76-0.84$ and for democratic decays $\varepsilon_{0} \approx 0.3-0.5$. Some uncertainty of the estimated $\varepsilon_{0}$ values here is connected with particular selection of parameter set for the system: $E_{T}, A, Z$, and $l$.

We note that the condition for the three-body decay 

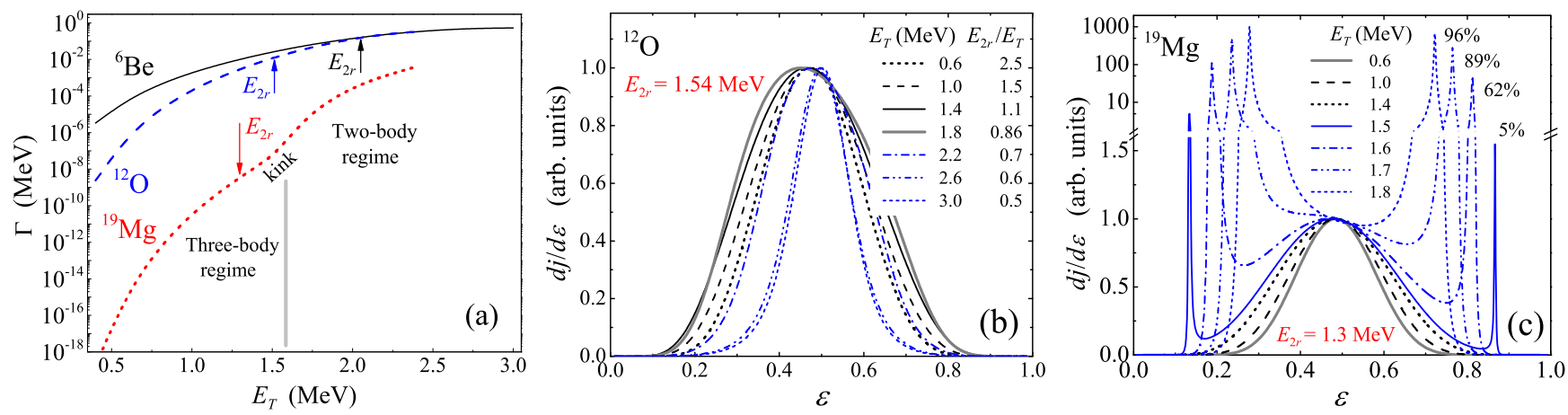

FIG. 34 (Color online) Transition from the true three-body to the sequential decay for ${ }^{6} \mathrm{Be},{ }^{12} \mathrm{O}$, and ${ }^{19} \mathrm{Mg}$ in the direct decay approximation Eq. 29p. The width vs. decay energy $E_{T}$ (a), evolution of energy distributions in the "Y" Jacobi system (core- $p$ channel) for the case of democratic decay (b), and for radioactive decay (c). The curves in (b,c) are normalized to unity for the maximum of the "bell" profile at $\varepsilon \approx 0.5$.

proposed by Goldansky (1960) was more restrictive:

$$
E_{T}+\Gamma_{2 r} / 2<E_{2 r}
$$

See also (Álvarez-Rodríguez et al. 2008a) for another formulation of how sequential and direct three-body decays may be distinguished.

\section{Diproton model}

Historically the first idea of the diproton approximation appeared in the original work of Goldansky (1960). Later, it was investigated in more detail (Goldansky, 1961) and gradually it has become one of the major ideas about the mechanism of the true $2 p$ decay, frequently used as a synonym of it. In this approach a single "diproton" particle (two protons in $l=0, S=0$ state) is tunneling through the barrier along the straight classical trajectory. The width in the "T" system is written as:

$\Gamma_{\mathrm{dp}}\left(E_{T}\right)=2 \gamma_{\mathrm{dp}}^{2} \int_{0}^{1} d \varepsilon \rho\left(\varepsilon E_{T}\right) P_{0}\left(E_{T}(1-\varepsilon), R_{\mathrm{dp}}, 2 Z_{\mathrm{core}}\right)$,

where the typical definitions of the diproton channel radius (Barker, 2001) is

$$
R_{\mathrm{dp}}=r_{0}\left(A_{1}^{1 / 3}+2^{1 / 3}\right),
$$

with $r_{0}=1.45$ (Barker, 2001). Value $\gamma_{\mathrm{dp}}$ is defined in an ordinary R-matrix form (33). The function $\rho(E)$ is the "density of diproton states" typically used in several forms:

$$
\begin{aligned}
& \rho(E)=E \delta\left(E-E_{0}\right), \\
& \rho(E) \sim P_{0}\left(E, R_{\mathrm{pp}}, 1\right), \\
& \rho(E) \sim \sin ^{2}\left[\delta_{0}(E)\right] .
\end{aligned}
$$

Eq. (44) corresponds to a "fixed-energy diproton". It is evident that some energy should be contained in the internal motion of the $p-p$ subsystem. In the early works it was taken $E_{0} \approx 50-100 \mathrm{keV}$ (Goldansky, 1961; Jänecke, 1965). The diproton model was often used in a trivial form with $E_{0}=0$ (Brown, 1991; Nazarewicz et al., 1996; Ormand, 1997). The results with $E_{0}=0$ for different $r_{0}$ values for ${ }^{45} \mathrm{Fe}$ are given in Fig. 28 (a). These results strongly overestimate $2 p$ decay width and could be considered as its strict upper limit. Eq. 45 corresponds to the "Coulomb-corrected phase volume". A radius of the $p-p$ channel of $R_{\mathrm{pp}} \sim 1-2 \mathrm{fm}$ should be chosen here to reproduce reasonably the low-energy $p-p$ scattering proporties. A treatment of the density in the spirit of the Migdal-Watson approximation, Eq. (46), was proposed by Barker (2001). Here $\delta_{0}(E)$ is the phase shift for the $s$ wave $p-p$ scattering. Results of the approaches 45 and (46) can be well approximated by expression Eq. (44) with an appropriate choice of $E_{0}$.

The diproton model in the form 42 has been inferred; it has never been derived. To check the validity of this approach the "dynamic" diproton model was developed by Grigorenko and Zhukov (2007a) using the analytical GF Eq. (28) of the simplified three-body Hamiltonian with a diproton Ansatz. It was demonstrated that starting from the Hamiltonian level, the results cannot be reduced to Eq. (42) with (43). Thus, the diproton model is typically used in an inconsistent way. Correctly applied, the diproton model provides too small values of the width, see Fig. 28(a), "diproton GF" curve. Moreover, Eq. (42) leads to a single, narrow, low-energy peak in the energy distribution in the "T" system, which differs from the experimental distributions, see Figs. 27(c), 31.c), and 29(g). In addition, Eq. 42 provides an angle-independent decay probability in the " $\mathrm{T}$ " system in contradiction to the experimental findings.

\section{Three-body model of $2 p$ radioactivity}

In the three-cluster model of $2 p$ radioactivity introduced by Grigorenko et al. (2000, 2002) the three-body 
Schrödinger equation with the complex energy

$$
\left(H_{3}-E+i \Gamma / 2\right) \Psi_{3 \varkappa}^{(+)}=0
$$

is solved using hyperspherical harmonics (HH) method. The solution with outgoing boundary condition is found in this method as

$$
\Psi_{3 \varkappa, J M}^{(+)}\left(\rho, \Omega_{5}\right)=\rho^{-5 / 2} \sum_{K \gamma} \chi_{\varkappa, K \gamma}^{(+)}(\rho) \mathcal{J}_{K \gamma}^{J M}\left(\Omega_{5}\right) .
$$

The hypermomentum $\varkappa=\sqrt{2 M E}$ is the analogue of the ordinary momentum in the $\mathrm{HH}$ three-body approach and the hyperradius $\rho$ is a collective variable describing the "breathing" motion of the system

$$
\rho=\frac{A_{1} A_{2} A_{3}}{A_{1}+A_{2}+A_{3}}\left(\frac{\mathbf{r}_{12}^{2}}{A_{3}}+\frac{\mathbf{r}_{23}^{2}}{A_{1}}+\frac{\mathbf{r}_{31}^{2}}{A_{2}}\right) .
$$

The hyperspherical harmonics $\mathcal{J}_{K \gamma}$ are functions of the 5 -dimensional "solid angle" $\Omega_{5}=\left\{\theta_{\rho}, \Omega_{x}, \Omega_{y}\right\}$. Here $\Omega_{x}$ and $\Omega_{y}$ are ordinary solid angles of the Jacobi vectors $\mathbf{X}$ and $\mathbf{Y}$ and $\tan \left(\theta_{\rho}\right)=\sqrt{M_{x} / M_{y}} X / Y$.

For radioactivity problems with $\left|E_{T}\right| \gg \Gamma$ the following procedure was found to be reliable. First, the discrete spectrum problem

$$
\left(H_{3}-E_{T}\right) \tilde{\Psi}_{3 \mathrm{~b}}=0
$$

is considered with some box boundary conditions (e.g. zero or quasistationary) yielding the "box" WF $\tilde{\Psi}_{3 \mathrm{~b}}$ and the value of the real resonant energy $E_{T}$. Then the inhomogeneous equation

$$
\left(H_{3}-E_{T}\right) \Psi_{3 \varkappa}^{(+)}=-i \Gamma / 2 \tilde{\Psi}_{3 \mathrm{~b}}
$$

is solved with arbitrary $\Gamma$ and the actual width is defined afterwards as the flux through a hypersphere of large radius divided by the normalization within this radius:

$$
\Gamma=\frac{j}{N}=\frac{\left.\operatorname{Im}\left[\int d \Omega_{5} \Psi_{3 \varkappa}^{(+) *} \rho^{5 / 2} \frac{d}{d \rho} \rho^{5 / 2} \Psi_{3 \varkappa}^{(+)}\right]\right|_{\rho=\rho_{\max }}}{M \int d \Omega_{5} \int_{0}^{\rho_{\max }} \rho^{5} d \rho\left|\Psi_{3 \varkappa}^{(+)}\right|^{2}}
$$

Formula 52 is intuitive. Still, some formal issues connected with its derivation can be found in Ref. (Grigorenko and Zhukov, 2007a)

The asymptotic form of the three-body potentials in the hyperspherical harmonics method is

$$
V_{K \gamma, K^{\prime} \gamma^{\prime}}(\rho)=\frac{U_{K \gamma, K^{\prime} \gamma^{\prime}}}{\rho^{N_{K \gamma, K^{\prime} \gamma^{\prime}}}}+\frac{C_{K}}{\rho^{2}} \delta_{K \gamma, K^{\prime} \gamma^{\prime}}+\frac{\varkappa \eta_{K \gamma, K^{\prime} \gamma^{\prime}}}{M \rho},
$$

where the multiindex $\{K \gamma\}=\left\{K, L, S, l_{x}, l_{y}, s_{x}\right\}$ is a complete set of quantum numbers for three clusters. The matrix $U_{K \gamma, K^{\prime} \gamma^{\prime}}$ arises due to contributions from the short-range nuclear forces, and $N_{K \gamma, K^{\prime} \gamma^{\prime}} \geq 3$ are integers: the effective contribution of the short-range forces decreases as $\rho^{-3}$ or faster in hypersherical space. The diagonal centrifugal term $C_{K}=\mathcal{L}_{K}\left(\mathcal{L}_{K}+1\right)$ depends on the "effective angular momentum" $\mathcal{L}_{K}=K+3 / 2$. Coulomb pairwise potentials generate the long-range part of the hyperspherical potentials behaving as $\rho^{-1}$. From the technical side, these three-body hyperspherical "Coulomb like" potentials cause problems due to long-range channel coupling (nondiagonal "Sommerfeld parameters" $\eta_{K \gamma, K^{\prime} \gamma^{\prime}}$ ) that does not allow one to decouple the $\mathrm{HH}$ equations in the asymptotic region. To deal with this problem, the finite-size potential matrix (in truncated hyperspherical basis) can be diagonalized with respect to the long-range term by the orthogonal transformation $\tilde{V}=A^{T} V A$ :

$$
\tilde{V}_{K \gamma, K^{\prime} \gamma^{\prime}}(\rho)=\frac{\tilde{U}_{K \gamma, K^{\prime} \gamma^{\prime}}}{\rho^{3}}+\frac{C_{K \gamma, K^{\prime} \gamma^{\prime}}}{\rho^{2}}+\frac{\varkappa \eta_{K \gamma}}{M \rho} \delta_{K \gamma, K^{\prime} \gamma^{\prime}} .
$$

This potential includes nondiagonal "centrifugal" terms $C_{K \gamma, K^{\prime} \gamma^{\prime}}$ and, to achieve the asymptotics in the diagonalized representation, we still need to go very far in $\rho$, where the terms $\sim \rho^{-2}$ become negligible compared to those with $\sim \rho^{-1}$. At such values of $\rho$, the hyperradial part of the asymptotic solution with pure outgoing nature can be constructed using the Coulomb functions:

$$
\begin{aligned}
\chi_{\varkappa, K \gamma}^{(+)}(\rho) & \sim \sum_{K^{\prime} \gamma^{\prime}} A_{K \gamma, K^{\prime} \gamma^{\prime}} \\
& \times\left[G_{\mathcal{L}_{0}}\left(\eta_{K^{\prime} \gamma^{\prime}}, \varkappa \rho\right)+i F_{\mathcal{L}_{0}}\left(\eta_{K^{\prime} \gamma^{\prime}}, \varkappa \rho\right)\right] .
\end{aligned}
$$

These boundary conditions are exact in the truncated $\mathrm{HH}$ basis at a hypersphere of very large radius, depending on energy and basis size. For example, for ${ }^{45} \mathrm{Fe}$ with $E_{T}=1.154 \mathrm{MeV}$ and $K_{\max }=20$, radii $\rho_{\max }$ between 500 and $2000 \mathrm{fm}$ are needed to get reasonable solutions.

The Coulomb three-body decays of nuclei have also been studied by other $\mathrm{HH}$ based theoretical methods. The S-matrix approach was used in calculations of ${ }^{6} \mathrm{Be}$ g.s. in Refs. (Danilin and Zhukov, 1993; Descouvemont et al. 2006 Vasilevsky et al. 2001) and ${ }^{12} \mathrm{O}$ g.s. in Ref. (Grigorenko et al., 2002). WKB calculations with adiabatic $\mathrm{HH}$ potentials were done for the width of the first excited state of ${ }^{17} \mathrm{Ne}$ in (Garrido et al., 2008). Having comparable quality for internal structure, these methods may have deficiencies dealing specifically with radioactivity problems: S-matrix calculations are difficult for very narrow states and WKB can not provide momentum distributions. We note that other $\mathrm{HH}$ based methods have been used to calculate the momentum distributions from three-body decay of several excited states in light nuclei (Álvarez-Rodríguez et al. 2010, 2008b.

\section{Lifetimes}

The Coulomb and $p-p$ potentials are the known ingredients of the three-cluster calculations. In the light nuclei, 
the core- $p$ potentials are fixed by fitting to the experimental single-particle spectra of the core- $p$ subsystem. In most cases this allows to obtain a reasonable description of the energy and structure of the ground and lowest excited states. For heavier nuclei, where the single particle description is not well justified and spectra of subsystems are often not known experimentally, certain systematic guidelines were proposed by Grigorenko et al. (2003a). Choosing the depths of potential components with different $l$ values we can vary the structure of the $2 p$ emitter for a fixed proton decay energy $E_{2 r}$ in the core- $p$ subsystem. A short-range (in the hyperradius) three-cluster potential is used to vary the decay energy $E_{T}$.

For the majority of prospective $2 p$ emitters the decay energies are not known. Therefore, the lifetime predictions are provided in terms of possible lifetime bands bound from above and below by calculations with pure configurations. The calculations for the $p$ - $f$ shell nucleus ${ }^{45} \mathrm{Fe}$ for different cases of configuration mixing are provided in Fig. 28. It can be seen that within the threebody model the precise lifetime data can be used to extract structural information about $2 p$ emitters.

A broader view of the true $2 p$ decay phenomenon is provided in Fig. 35. For the light $2 p$ emitters specific lifetimes are predicted (gray circles). For the heavier $2 p$ emitters the predicted lifetime "bands" are in a good agreement with the experimental data. The lifetime range for the known true $2 p$ emitters spans about 18 orders of the magnitude. This plot emphasizes the complexity of the problem requiring a variety of experimental methods to cover the possible lifetime range.

\section{Spatial correlations}

The evolution of spatial correlations in the three-body model is illustrated by the case of the ${ }^{45} \mathrm{Fe}$ structure with $W\left(p^{2}\right)=98 \%$, see Fig. 36. In the Jacobi "T" system, this case of almost pure $\left[f^{2}\right]_{0}$ configuration has a very eminent correlation pattern with four peaks in the internal region. Such correlations can be related to the so-called Pauli focusing - counting of excitation quanta in the "T" system.

It can be seen in Fig. 36 that under the barrier the four-hump structure is dissolved and a different correlation pattern is formed while transition to the asymptotic region takes place. Finally, the momentum distribution with one peak in energy between two protons is formed, see Fig. 37(b). This happens due to $p-p$ interaction in the subbarrier region (Grigorenko et al. 2000). It is evident here that the penetration process influences the distribution strongly and the information about correlations in the nuclear interior is present in the final momentum distributions in a very indirect way. Detailed theoretical calculations of the subbarrier propagation process are re-

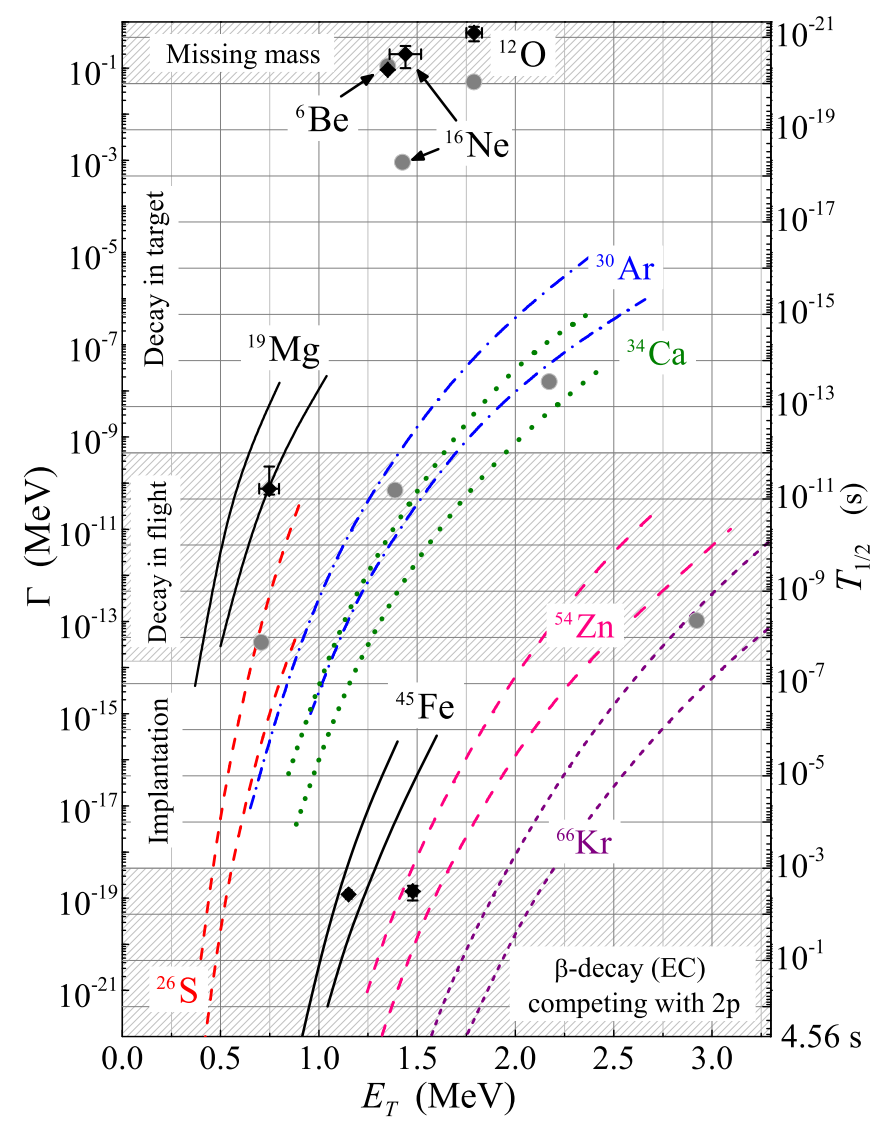

FIG. 35 (Color online) Lifetime vs. decay energy systematics for several known and prospective true $2 p$ emitters calculated in three-body model. Hatching indicates lifetime ranges accessible to different experimental techniques. Experimental results are shown by diamonds. Gray circles show specific predictions, where available.

quired.

\section{Momentum correlations and nuclear structure}

The correlation pictures calculated in the three-body model are provided in Figs. 27(a,b), 31(a,b), and 29(e,f) for ${ }^{6} \mathrm{Be},{ }^{19} \mathrm{Mg}$ and ${ }^{45} \mathrm{Fe}$, respectively. Despite the strong differences there are certain common features in these distributions.

(i) There are Coulomb suppression regions due to $p-p$ repulsion at $\varepsilon \sim 0$ in the " $\mathrm{T}$ " system and at $\varepsilon \sim 0.5$, $\cos \left(\theta_{k}\right) \sim-1$ in the "Y" system. Analogous regions due to core- $p$ repulsion appear at $\varepsilon \sim 0.5, \cos \left(\theta_{k}\right) \sim \pm 1$ in the "T" system and at $\varepsilon \sim 0, \varepsilon \sim 1$ in the "Y" system. These regions grow drastically as we move from light to heavier $2 p$ emitters.

(ii) In the "Y" system the particles are concentrated around $\varepsilon \sim 0.5$. This effect becomes more pronounced as the core charge increases. This particular aspect of the correlation picture is consistent with the original predic- 

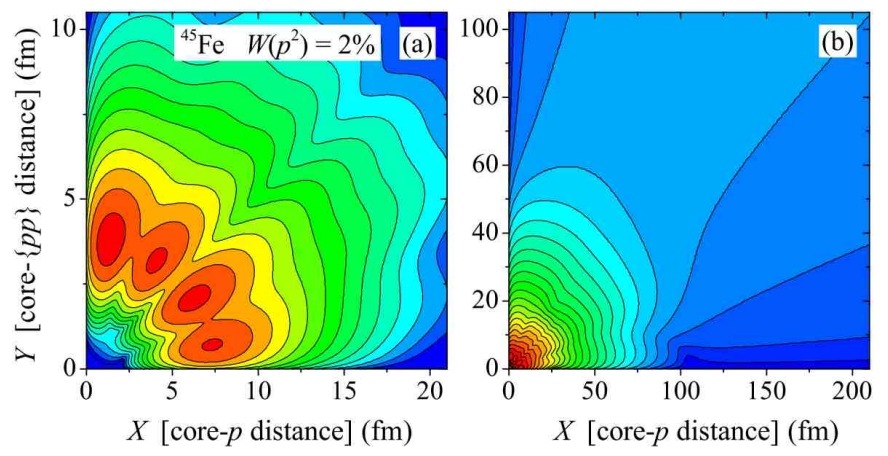

FIG. 36 (Color online) Spatial correlations in the ${ }^{45} \mathrm{Fe}$ WF in the "T" system for $W\left(p^{2}\right)=2 \%$. Panels (a) and (b) shows different radial ranges. Logarithmic scale: two (a) and one (b) contours per order of the magnitude.

tions of Goldansky and with calculations performed in a simplified direct decay model, see Fig. 32 . In the "T" system this effect appears as a concentration of particles in the T-like configuration with $\cos \left(\theta_{k}\right) \sim 0$.

(iii) The $p$ - $p$ final-state interaction gives rise to enhancements at small values of $\varepsilon$ in the "T" system. This enhancement varies from minor to a very expressed one depending on the particular dynamics of the system, see also Fig. 37 .

An important question about $2 p$ radioactivity asked from the earliest days of this research is "Which kind of information about internal structure can be extracted from the $2 p$ decay data?" It was not clear in advance that information about the nuclear interior would survive in the process of penetration of protons through the Coulomb barrier. The calculations in the three-body model have demonstrated that correlations in the $p-p$ channel are sufficiently sensitive to nuclear structure to be discussed as prospective instrument for nuclear spectroscopy in both $s-d$ and $p$ - $f$ shell nuclei, see Fig. 37. These correlations are manifested by the $\varepsilon$ variable in the "T" system and the $\cos \left(\theta_{k}\right)$ variable in the "Y" system (Figs. 27 and 29).

\section{Long-range character of Coulomb interaction in the three-body continuum}

The formulation of approximate boundary conditions Eq. 55) is satisfactory for distances around $10^{3} \mathrm{fm}$. Such distances appear to be insufficient to get converged momentum distributions for heavy $2 p$ emitters. Extrapolation by means of classical trajectories was used by Grigorenko et al. (2010) to improve the momentum distributions. Classical extrapolation modifies mainly the $\cos \left(\theta_{k}\right)$ distribution in "T" system and the $\varepsilon$ distribution in "Y" system, see Fig. 38(a,b). The effect of classical extrapolation is important already on the current level of experimental precision, Fig. 38(c). A huge range is required
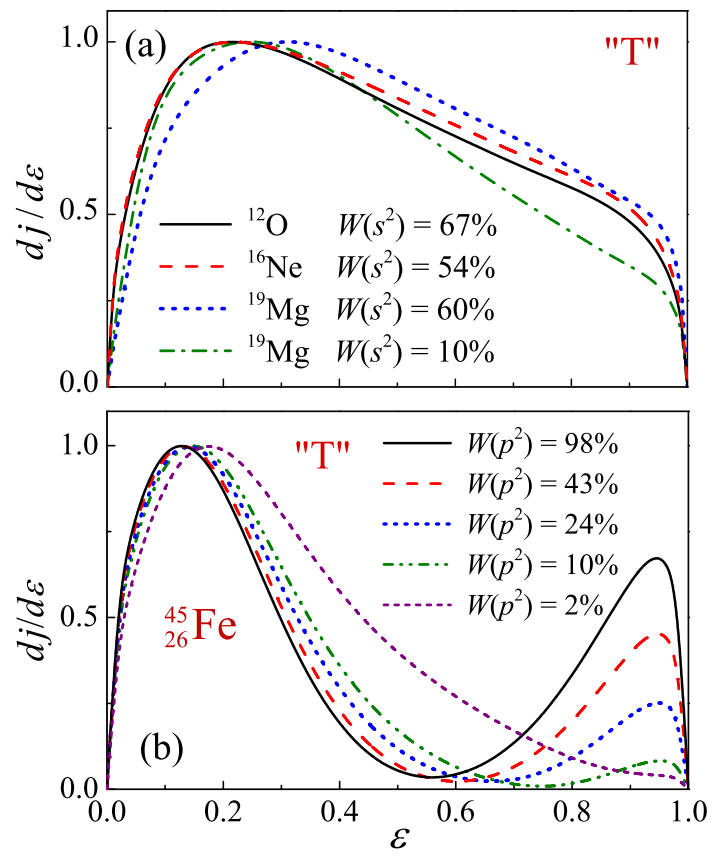

FIG. 37 (Color online) The energy distributions between two protons ("T" Jacobi system) in different $s$ - $d$ shell nuclei (a) and with different assumptions about the internal structure of ${ }^{45} \mathrm{Fe}(\mathrm{b}) . W\left(l^{2}\right)$ is the weight of the $\left[l^{2}\right]$ configuration in the nuclear interior. All distributions are normalized to unity maximum value. From (Grigorenko and Zhukov, 2003b).

both for the extrapolation range $\left(\sim 10^{5} \mathrm{fm}\right)$ and for the starting point of the classical procedure $\left(\sim 10^{3} \mathrm{fm}\right)$ under typical $2 p$ decay conditions. The maximal extrapolation radius is comparable to atomic distances and sensitivity of $2 p$ momentum distributions to atomic screening of nuclear Coulomb potential can be found. This brings the $2 p$ decays to the borderline with atomic phenomena.

\section{E. Two-proton radioactivity and many-body nuclear structure}

A very important aspect of $2 p$ radioactivity studies is the understanding of the connection between specific asymptotic observables ( $2 p$ correlations) and the WF structure in the internal region (nuclear spectroscopy). In Sec. VII.D.3 such a connection was demonstrated for the three-body model of true $2 p$ decay. However, the discussed three-body approach should be regarded as a first approximation model of this process. By analogy to two-body decay, the exponential component of the width is expected to be treated appropriately in this method. The three-cluster model accounts well for single-particle degrees of freedom in the core- $p$ channel. Therefore, calculation of the "preexponent" in three-cluster model is well justified only for the light closed-shell (closedsubshell) systems or systems with closed-shell (closedsubshell) core. With increasing mass number fewer systems can be found along the dripline whose dynamics can 

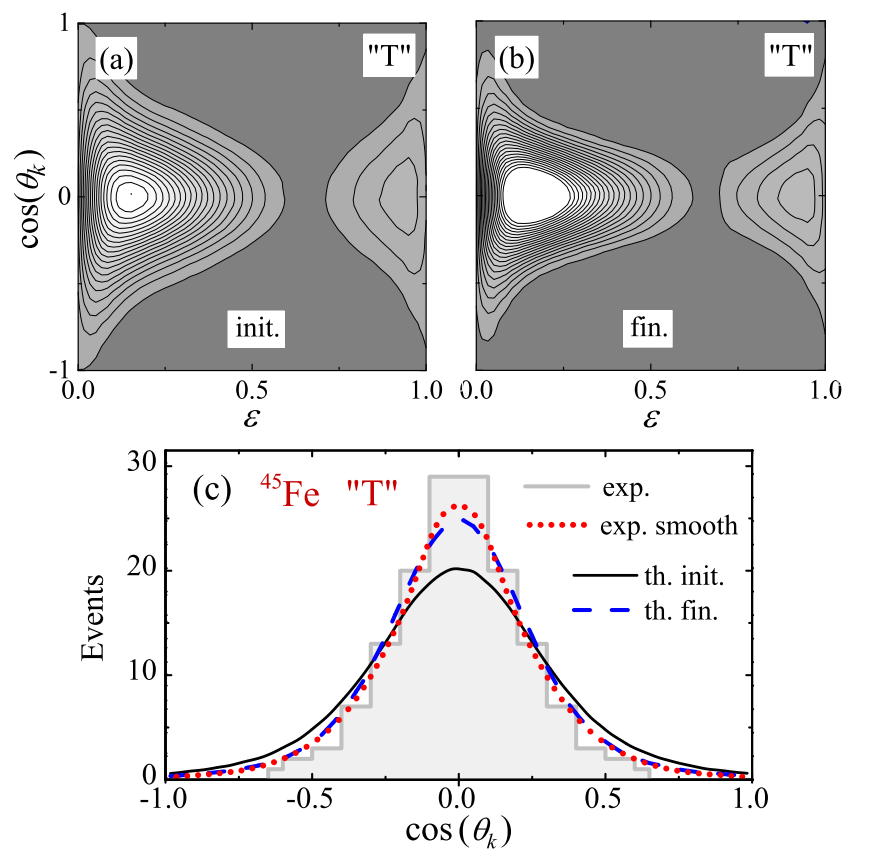

FIG. 38 Contour maps of the momentum density distribution on the kinematical plane $\left\{\varepsilon, \cos \left(\theta_{k}\right)\right\}$ for ${ }^{45} \mathrm{Fe}$ in the "T" Jacobi coordinate system without (a) and with (b) classical extrapolation. Panel (c) shows comparison with experimental data of (Miernik et al. 2007c). Experimental and theoretical events are convoluted with the experimental resolution. From (Grigorenko et al. 2010).

be reasonably described in the three-cluster approximation. Taking many-body effects into account in the $2 p$ decay calculations is a natural next step in the studies of the phenomenon. Here, we briefly sketch several possible approaches to this problem which are being considered presently.

\section{Spectroscopic information in R-matrix approaches}

The information about many body structure is embedded in the simplified models of two proton radioactivity via spectroscopic factors. The single particle proton factors $\theta^{2}$ Eq. 31) were used for this purpose within the direct decay model Eq. (37) (Barker, 1999, 2003, Brown et al. 2002, Kryger et al., 1995).

The diproton spectroscopic factors [for use within the diproton model Eq. [42] ] are obtained by projecting WFs of valence protons on the "diproton quantum numbers" for two protons (relative angular momentum zero and total spin zero). It is also typically assumed that the relative motion of the protons is represented by the lowest oscillator. Calculations of this type can be found in Refs. (Barker, 2003, Brown, 1991; Brown et al., 2002).

\section{Three-body model plus RMF amplitudes}

A simple method of structure treatment in the threecluster model was proposed by Fomichev et al. (2011). This approach is equivalent to a standard method used in the R-matrix description of two-body decays. The major components of the three-body cluster WFs with $J^{\pi}=0^{+}$ can be written in a schematic spectroscopic notation as

$$
\Psi_{3}^{(+)}=\sum_{i} X_{i}\left[l_{i}^{2}\right]_{0}
$$

The values which can be put in correspondence with the components of the three-body WF Eq. (56), to take into account the many-body structure, are overlaps of the many-body WFs of the precursor-daughter pair multiplied by a combinatorial term. They can be written in the same spectroscopic notation:

$$
\left(\frac{A !}{2 !(A-2) !}\right)^{1 / 2}\left\langle\Psi_{A} \mid \Psi_{A-2}\right\rangle=\sum_{i} \tilde{X}_{i}\left[l_{i}^{2}\right]_{0} .
$$

For WFs normalized to unity in the internal region the amplitudes of overlaps do not sum to unity in the general case in contrast with amplitudes of components:

$$
\sum_{i}\left|X_{i}\right|^{2} \equiv 1, \quad N_{2 p}=\sum_{i}\left|\tilde{X}_{i}\right|^{2} \neq 1
$$

Therefore the three-body width as a function of weights for all considered single-particle configurations $\left\{X_{i}^{2}\right\}=$ $\left\{X_{1}^{2}, \ldots, X_{n}^{2}\right\}$ should be renormalized as:

$$
\Gamma\left(\left\{X_{i}^{2}\right\}\right) \rightarrow N_{2 p} \Gamma\left(\left\{\tilde{X}_{i}^{2} / N_{2 p}\right\}\right) .
$$

For systems which are not closed-shell/closed-subshell, the coefficient $N_{2 p}$ is considerably different from unity already in the Hartree approximation. The higher complexity of the three-body decays is reflected in the fact that inclusion of structure information can not be reduced to multiplication by some factor, but should precede three-body calculations.

An advantage of the method is the opportunity to use relatively standard input from many-body theory, Eq. (57). For example, Fomichev et al. (2011) calculated these amplitudes in the relativistic mean field model (Litvinova et al. 2008). A disadvantage is the different treatment of the internal structure and the decay process. The same problem, however, exists also in the conventional two-body R-matrix phenomenology. The center of mass is treated appropriately in the three-body decay calculations, but only effectively in the many-body part.

\section{Shell model approaches}

Several shell-model approaches have been developed for studies of two-nucleon decays.

The Gamow shell model is based on the utilization of the so-called Berggren basis (Michel et al. 2003). This 
is a basis composed of Gamow states - single-particle states with decay asymptotic $\sim e^{i k r}$. For homogeneous Schrödinger equations such states should have complex energies. The eigenenergy of the shell-model Hamiltonian diagonalized in such a basis is naturally complex as well, with the imaginary part interpreted as a halfwidth. Technicalities make application of this method problematic for extremely narrow states. So far, it has been applied to light neutron-rich systems (Michel et al. 2010a, and Refs. therein).

The real-energy continuum shell model was employed for studies of narrow states decaying by two-nucleon emission by Rotureau et al. (2005, 2006) and Volya and Zelevinsky (2006). In this approach the Hamiltonian is split in two parts

$$
H=H_{\mathcal{Q Q}}+H_{\mathcal{Q P}}+H_{\mathcal{P} \mathcal{Q}}+H_{\mathcal{P P}} .
$$

Functional subspaces $\mathcal{Q}$ and $\mathcal{P}$ can be identified as "internal region" with discretized (shell-model) basis $\phi_{i}$ and "external region" with continuum basis $\phi_{E}$ defined by projection operators:

$$
\hat{\mathcal{Q}}=\sum_{i}\left|\phi_{i}\right\rangle\left\langle\phi_{i}\left|, \quad \hat{\mathcal{P}}=\int d E\right| \phi_{E}\right\rangle\left\langle\phi_{E}\right| .
$$

Elimination of the subspace $\mathcal{P}$ can be done by the Green's function methods leading to effective (energy dependent and non-Hermitian) Hamiltonian $\mathcal{H}$ in $\mathcal{Q}$

$$
\mathcal{H}_{\mathcal{Q Q}}(E)=H_{\mathcal{Q Q}}+H_{\mathcal{Q P}} G_{P}^{(+)}(E) H_{\mathcal{P} \mathcal{Q}}
$$

which allows straightforward application of powerful shell-model methods. An advantage of the continuum shell model is the use of extensive shell model expertise and applicability to corresponding broad range of nuclear systems.

The continuum shell model method of (Volya and Zelevinsky, 2006) was applied in extensive studies of the neutron-rich helium and oxygen isotopes providing good description of the known cases of $2 n$ decay and predictions for several unknown. In this approach the residual interaction in subspace $\mathcal{P}$ is neglected. Therefore, in the sense of the three-body continuum dynamics this model is analogous to the direct decay model, Sec. VII.C.1. It can be shown that the expression for the $2 n$ width from (Volya and Zelevinsky, 2006) can be approximated by Eq. (35).

The shell model embedded into continuum (SMEC) was applied by Rotureau et al. (2006) to studies of $2 p$ radioactivity using either the diproton or direct decay Ansatz. The later is called "sequential 2p emission" having in mind "emission via tails of higher-lying states", see also the discussion of Sec. VII.C.2 to avoid confusion of terminology. The calculated diproton lifetimes for ${ }^{45} \mathrm{Fe}$, ${ }^{48} \mathrm{Ni}$, and ${ }^{54} \mathrm{Zn}$ are typically exceeding the experimental values by a few times, see, e.g., Fig. 28 for ${ }^{45}$ Fe results.
A possible reason could be that the diproton approximation tend to underestimate width, see the discussion in Section VII.C.3 The direct decay lifetimes calculated for ${ }^{45} \mathrm{Fe}$ are $20-50$ times larger than experimental one, see Fig. 28.

A disadvantage of the shell-model methods is that no matching to the three-body boundary conditions was realized so far and calculations of momentum distributions for decay products are not possible. The necessity to incorporate three-body asymptotics in the SMEC calculations was emphasized in (Blank and Płoszajczak, 2008; Rotureau et al., 2006). The formalism for this was proposed in (Rotureau et al. 2006) using hyperspherical decomposition (48) and boundary conditions (55). The obstacle, which Rotureau et al. (2006) faced here is in the contact character of the residual interaction in SMEC. Delta-function interactions lead to collapse of three-body calculations. Use of finite-range residual interaction is technically complicated in SMEC (Rotureau et al. 2006).

An important conceptual problem exists that should be common to all shell-model approaches. The asymptotic interaction in the $p-p$ channel (in the $\mathcal{P}$ subspace) should be the "vacuum" $p-p$ potential. This should be reconciled in the internal region with the shell-model residual interaction which has effective character, reflecting many-body dynamics of nuclear interior. This reconciliation should take place somewhere close to the nuclear surface. The observables for $2 p$ decay are especially sensitive to this radial range, see Sec. VII.D.2, making it an important issue.

\section{Microscopic cluster models}

Microscopic cluster models do not have the conceptual problem mentioned in the end of the previous section utilizing the same (quasi) realistic nucleon-nucleon interactions in all space. Microscopic resonating group methods (RGM) have been applied to the lightest true $2 p$ emitter ${ }^{6} \mathrm{Be}$.

The complex scaling method of (Csótó, 1994) was the first example of such studies. In this method the boundary conditions are not explicitly formulated and therefore only resonance widths can be calculated.

Algebraic version of RGM (Nesterov et al., 2010, Vasilevsky et al., 2001) for three-cluster systems use WF in the form

$$
\Psi(A)=\mathcal{A}\left[\Psi\left(A_{1}\right) \Psi\left(A_{2}\right) \Psi\left(A_{3}\right) \Psi_{3 \varkappa}\left(\rho, \Omega_{5}\right)\right],
$$

where $\Psi\left(A_{i}\right)$ are cluster WFs, $A=A_{1}+A_{2}+A_{3}$, and $\Psi_{3 \varkappa}$ is three-body scattering WF in the "S-matrix representation" (three-body plane wave plus three-body outgoing wave). The derived S-matrix is diagonalized and the matrix elements with the most expressed resonance behavior ("eigenphases") are used to extract resonance energy and 
width;

$$
d^{2} \delta(E) /\left.d E^{2}\right|_{E=E_{T}}=0, \quad \Gamma=\left.2[d \delta(E) / d E]^{-1}\right|_{E=E_{T}} .
$$

A hyperspherical decomposition was used for WF $\Psi_{3 \varkappa}$ as especially suitable for imposing long-range boundary conditions for true three-body decays. A somewhat simpler version of the boundary conditions than (55) was realized in (Vasilevsky et al. . 2001) with diagonal terms only retained in the Coulomb term of Eq. (53). The algebraic method employs two different hyperspherical basis sets for the nuclear interior and asymptotic providing a natural and controllable treatment of these two regions. The disadvantage is that the calculations already for ${ }^{6} \mathrm{Be}$ seem to be not sufficiently converged at the present level of sophistication compared to fully converged pure threebody calculations (Grigorenko et al., 2009a).

A general challenge for microscopic cluster models is that realistic calculations can be performed only for the lightest systems with effective nucleon-nucleon interactions typically employed. However, modern trend is to move to heavier systems and realistic interactions.

\section{F. Three and four proton emission}

The beta-delayed three-proton decay of ${ }^{45} \mathrm{Fe}$ was reported by Miernik et al. (2007b). Most likely this is a sequential decay and it has been discussed already in Sec. IV. Three-proton decay of highly excited states of ${ }^{16} \mathrm{Ne}$, populated in fragmentation reaction, via narrow intermediate states in ${ }^{15} \mathrm{~F}$ and ${ }^{14} \mathrm{O}$, was observed by Mukha et al. (2009). Theoretical prospects of studies of this decay branch are so far unclear.

Following the analogy with the true $2 p$ emission ( $1 p$ emission is energetically prohibited) we can define the true $4 p$ emission (emission of $i$ protons is energetically prohibited for all $i<4$ ). The only candidate to fulfil this condition studied so far is ${ }^{8} \mathrm{C}$. It was shown recently that it is not a true $4 p$ emitter (Charity et al., 2010). This nucleus undergoes a sequence of true $2 p$ emissions decaying via the ${ }^{6} \mathrm{Be}$ ground state. The next candidate for the true $4 p$ emitter is ${ }^{21} \mathrm{Si}$ but no information is available for this isotope yet.

\section{EMISSION OF NEUTRONS}

With the progress in reaching experimentally the neutron drip-line, the interest to study nuclei beyond this limit is rising. Emission of protons beyond the proton drip-line has an analogue in neutron(s) emission beyond the neutron drip-line. In this Section we want to comment on the possibility of neutron(s) emission which may take the form of neutron/few-neutron radioactivity. Some aspects of such processes were discussed by Thoennessen (2004).

For illustration we consider theoretical estimates for the two pairs: ${ }^{26} \mathrm{~S}-{ }^{26} \mathrm{O}$ and ${ }^{25} \mathrm{P}_{-}{ }^{25} \mathrm{O}$. They are isobaric partners but not mirror nuclei (the $\{Z, N\}$ values are $\{16,10\}-\{8,18\}$ and $\{15,10\}-\{8,17\})$. They have similar mass/charge ratios, opened $s$ - $d$ shell, and should presumably have comparable structural and radial characteristics. ${ }^{26} \mathrm{~S}$ is a candidate to be a true $2 p$ emitter and ${ }^{26} \mathrm{O}$ could be a narrow two-neutron resonance.

For the one-neutron emission, simple estimates can be obtained by the standard R-matrix expressions, Eqs. (31) and (32). The results for protons and neutrons are shown in Fig. 39(a). Assuming a possible $s$ - or $d$-wave ground state for $A=25$, one can see that the decay energy window corresponding to proton radioactivity ranges from $\sim 50$ to $\sim 200 \mathrm{keV}$. In contrast, for the neutron-emitting partner $\left({ }^{25} \mathrm{O}\right)$ to be classified as radioactive, the decay energy, even for the $d$-wave, would have to be smaller than $1 \mathrm{keV}$. It is highly improbable that such a fine-tuned energy is actually found. It seems that a realistic chance to observe one-neutron radioactivity may appear only for $f$ wave and higher- $l$ states. As long as the heavier neutron drip-line nuclei are not known, long-lived neutron emitters cannot be excluded. However, it is likely to happen beyond the $s-d$ shell.

The two-neutron radioactivity, or true two-neutron $(2 n)$ decay, is defined in analogy to the true $2 p$ decay (see Sec. VII.A as a simultaneous emission of two neutrons where one-neutron emission is energetically prohibited. The possible $2 n$ radioactivity, shown in Fig. 39(b), has a few important differences in comparison to the oneneutron radioactivity.

(i) Low-energy $s$-wave neutron emission could take place in the form of a virtual state, which can not be interpreted in terms of width (the neutron $s$-wave curve is missing in Fig. 39(a)). For the $2 n$ emission the phase space for a few-body final state creates additional effective centrifugal barrier, so that a narrow resonance state is formed even for the decay of a $\left[s^{2}\right]$ configuration. The possibility to observe narrow "three-body virtual states" build on $\left[s^{2}\right]$ configurations was discussed by Grigorenko and Zhukov (2008) for the case of ${ }^{10} \mathrm{He}$. The search for the $2 n$ ground-state decay has indicated only the existence of quite broad $(\Gamma \gtrsim 1 \mathrm{MeV})$ states in ${ }^{5} \mathrm{H},{ }^{10} \mathrm{He}$, and ${ }^{13} \mathrm{Li}$ (Golovkov et al. $, 2005,2009$; Johansson et al. 2010 Korsheninnikov et al., 1994). 

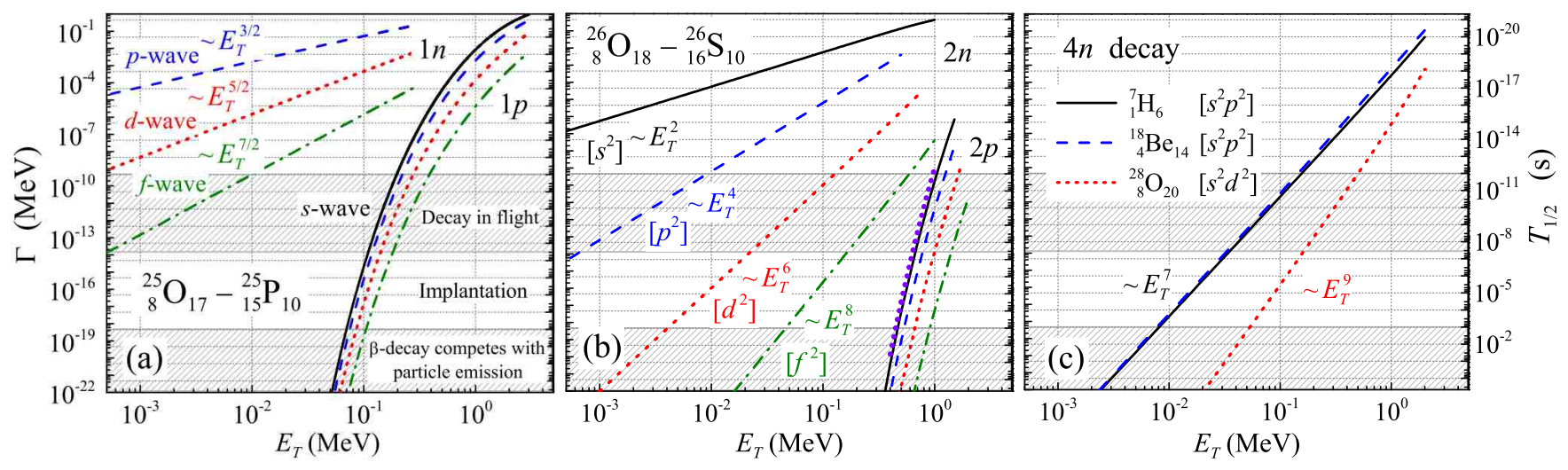

FIG. 39 (Color online) Estimated widths for the neutron (a) and the two-neutron emission (b) compared with widths for the proton and the two-proton emission. Panel (c) shows estimates for the four-neutron emission. Hatched areas indicate lifetime ranges accessible by different experimental techniques, see also Fig. 35.

(ii) Similarly to the $p-2 p$ decay comparison, the widths for the true two-neutron emission are much smaller than for the one-neutron emission with the same decay energy. The energy window for $2 p$ radioactivity of ${ }^{26} \mathrm{~S}$ extends up to $500-1700 \mathrm{keV}$, thus is about an order of magnitude larger than for the proton radioactivity of ${ }^{25} \mathrm{P}$. The estimated relative increase of the energy window for $2 n$ radioactivity compared to $1 n$ radioactivity is even larger (about two orders of magnitude). For example, for the $\left[d^{2}\right]$ and the $\left[f^{2}\right]$ configurations, the true $2 n$ decays would be classified as radioactive for decay energies ranging up to $\sim 200 \mathrm{keV}$ and $\sim 600 \mathrm{keV}$, respectively. Such broad ranges make the search for $2 n$ radioactivity much more promissing.

(iii) In contrast to the $1 n$ situation, the $2 n$ estimates in Fig. 39(b) should be interpreted as lifetime limits due to the possibility of configuration mixing. The $\left[s^{2}\right]$ and $\left[p^{2}\right]$ curves are likely to provide lower lifetime limits for $s$ - $d$ and $p$ - $f$ configurations, respectively. The $\left[d^{2}\right]$ and $\left[f^{2}\right]$ curves provide upper lifetime limits for them. From the experience collected in the $2 p$ decay studies we may argue that the realistic results are located between the limiting curves, but closer to that for the lower $l$.

The systems decaying by a simultaneous emission of more than two neutrons should have longer lifetimes for the given decay energy. The estimates of the true fourneutron decay width can be performed in the approximation generalizing the direct decay model of Eq. (29). For a true four-neutron decay this model gives (Grigorenko et al. 2011):

$$
\begin{aligned}
\Gamma_{\mathrm{dir}}\left(E_{T}\right) & =\frac{E_{T}^{3}\left(E_{T}-\sum_{i=1}^{4} E_{n_{i}}\right)^{2}}{2 \pi^{3}} \int_{0}^{1} d \varepsilon_{1} \int_{0}^{1-\varepsilon_{1}} d \varepsilon_{2} \\
& \times \int_{0}^{1-\varepsilon_{1}-\varepsilon_{2}} d \varepsilon_{3} \prod_{i=1}^{4} \frac{\Gamma_{n_{i}}\left(E_{i}\right)}{\left(E_{i}-E_{n_{i}}\right)^{2}+\Gamma_{n_{i}}\left(E_{i}\right)^{2} / 4} .
\end{aligned}
$$

Here $E_{i}=\varepsilon_{i} E_{T}$ for $i=1,2,3$ and $E_{4}=\left(1-\varepsilon_{1}-\varepsilon_{2}-\right.$ $\left.\varepsilon_{2}\right) E_{T}$. The $E_{n_{i}}$ and $\Gamma_{n_{i}}$ are energy and width of the lowest resonance in the core and $i$-th neutron subsystem.

The decay of ${ }^{7} \mathrm{H}$ is strongly suspected to proceed by a true four-neutron emission. The decay energy is uncertain, but seems to be smaller than $2 \mathrm{MeV}$ (Caamano et al., 2008; Korsheninnikov et al., 2003, Nikolskii et al., 2010). The ground-state decay energies of the subsystems: ${ }^{4} \mathrm{H}$ (Tilley et al. 1992 ) and ${ }^{5} \mathrm{H}$ (Golovkov et al. 2005) are around $2 \mathrm{MeV}$. There is only un upper limit of $1 \mathrm{~ns}$ for the lifetime of ${ }^{7} \mathrm{H}$ (Golovkov et al., 2004). The nearest heavier candidates for the true four-neutron emitters are ${ }^{18} \mathrm{Be}$ and ${ }^{28} \mathrm{O}$.

In Fig. 39(c) the estimates for the true four-neutron emission lifetime are shown for ${ }^{7} \mathrm{H},{ }^{18} \mathrm{Be}$, and ${ }^{28} \mathrm{O}$. For simplicity we used $E_{n_{i}}=1.5 \mathrm{MeV}$ in all cases. The other parameters are chosen according to guidelines from Sec. VII.C.1. The orbital configurations were chosen to yield results closer to the upper limit for the width, therefore, the provided estimates are conservative. These estimates indicate that long-living states decaying by true four-neutron emission are possible, with decay energies up to hundreds of $\mathrm{keV}$, even for $p$ and $s$ - $d$ shell nuclei.

In summary, the observation of neutron radioactivity in $s$ - $d$ shell nuclei seems unrealistic. It is more probable that this process occurs in heavier ( $p-f$ shell) systems. The discovery of $2 n$ or $4 n$ radioactivity is much more probable since the energy windows corresponding to the radioactive timescale are much broader. However, both $2 n$ and $4 n$ lifetimes depend strongly on the nuclear structure and quantitative predictions require further investigations. 


\section{CONCLUSIONS}

The exploration of the driplines of the nuclear chart have made great progress during the last decade. Our knowledge on nuclei along and beyond the proton dripline has increased significantly and studies of the neutron dripline has now reached the Ne- $\mathrm{Mg}$ region. New radioactive decay modes appear here, namely one-proton and two-proton emission from long-lived states as well as many beta-delayed particle emission branches. This review has given an overview of the present state of the field.

Beta-delayed particle emission will be prominent at the driplines and many modes will mainly occur there, the exception being beta-delayed one- and multi-neutron emission that is expected to play a major role for neutron-rich nuclei, in particular in the still unexplored part of the nuclear chart.

The single-proton emission, be it as radioactivity or following a beta-decay, is now well understood and established as a valuable probe of nuclear structure evolution, in close analogy to the role that alpha decay has played.

Two-proton radioactivity is the most recently discovered decay mode. It is as well sensitive to nuclear structure, but there are interesting physics questions in the emission process itself that are not fully clarified and are intimately related to the more general problem of three-body break-up. To some extent this also holds for beta-delayed multi-particle decays, where only a few cases have been thoroughly explored experimentally.

In all the cases mentioned theoretical and experimental activities are ongoing in order to tackle the important questions that await clarification. An essential ingredient in answering these questions is the present rapid developments in the production of secondary beams of neardripline isotopes and the corresponding continuing evolution of experimental techniques. Along with dedicated theoretical efforts this should ensure that the progress continues.

\section{ACKNOWLEDGMENTS}

We are grateful to many colleagues for discussions and would like to thank in particular H.O.U. Fynbo, G. Martínez-Pinedo, I. Mukha, G. Ter-Akopian, M.V. Zhukov, and J. Żylicz. This work was supported in part by FAIR-Russia research center (LVG).

\section{REFERENCES}

Åberg, S., P. Semmes, and W. Nazarewicz, 1997, Phys. Rev. C 56, 17762 .

Abramowitz, M., and I. A. Stegun, 1968, Handbook of mathematical functions (Dover, New-York).

Achouri, N., F. de Oliveira Santos, M. Lewitowicz, B. Blank, J. Äystö, G. Canchel, S. Czajkowski, P. Dendooven, A. Emsallem, J. Giovinazzo, N. Guillet, A. Jokinen, et al., 2006, The European Physical Journal A - Hadrons and Nuclei 27, 287.

Adimi, N., R. Domínguez-Reyes, M. Alcorta, A. Bey, B. Blank, M. J. G. Borge, F. d. O. Santos, C. Dossat, H. O. U. Fynbo, J. Giovinazzo, H. H. Knudsen, M. Madurga, et al., 2010, Phys. Rev. C 81(2), 024311.

Akovali, Y., 1998, Nucl. Data Sheets 84, 1.

Algora, A., D. Jordan, J. L. Taín, B. Rubio, J. Agramunt, A. B. Perez-Cerdan, F. Molina, L. Caballero, E. Nácher, A. Krasznahorkay, M. D. Hunyadi, J. Gulyás, et al., 2010, Phys. Rev. Lett. 105(20), 202501.

Alvarez, L., 1937, Phys. Rev. 52(2), 134.

Álvarez-Rodríguez, R., H. O. U. Fynbo, A. S. Jensen, and E. Garrido, 2008a, Phys. Rev. Lett. 100(19), 192501.

Álvarez-Rodríguez, R., A. S. Jensen, E. Garrido, and D. V. Fedorov, 2010, Phys. Rev. C 82(3), 034001.

Álvarez-Rodríguez, R., A. S. Jensen, E. Garrido, D. V. Fedorov, and H. O. U. Fynbo, 2008b, Phys. Rev. C 77(6), 064305.

Andreyev, A., D. Ackermann, F. Heßberger, K. Heyde, S. Hofmann, M. Huyse, D. Karlgren, I. Kojouharov, B. Kindler, B. Lommel, G. Münzenberg, R. Page, et al., 2004, Phys. Rev. C 69, 054308.

Andreyev, A., M. Huyse, P. Duppen, L. Weissman, D. Ackermann, J. Gerl, F. Heßberger, S. Hofmann, A. Kleinböhl, G. Münzenberg, S. Reshitko, C. Schlegel, et al., 2000, Nature 405, 430.

Andreyev, A. N., D. Ackermann, S. Antalic, I. G. Darby, S. Franchoo, F. P. Heßberger, S. Hofmann, M. Huyse, P. Kuusiniemi, B. Lommel, B. Kindler, R. Mann, et al., 2005, Phys. Rev. C 72(1), 014612.

Andreyev, A. N., J. Elseviers, M. Huyse, P. Van Duppen, S. Antalic, A. Barzakh, N. Bree, T. E. Cocolios, V. F. Comas, J. Diriken, D. Fedorov, V. Fedosseev, et al., 2010, Phys. Rev. Lett. 105(25), 252502.

Angélique, J. C., C. Timis, S. Piétri, N. L. Achouri, P. Baumann, C. Borcea, A. Buta, W. Catford, S. Courtin, J. M. Daugas, P. Dessagne, F. de Oliveira, et al., 2006, AIP Conference Proceedings 831(1), 134.

Anthony, D., L. Buchmann, P. Bergbusch, J. M. D'Auria, M. Dombsky, U. Giesen, K. P. Jackson, J. D. King, J. Powell, and F. C. Barker, 2002, Phys. Rev. C 65(3), 034310.

Antony, M. S., A. Pape, and J. Britz, 1997, Atomic Data and Nuclear Data Tables 66(1), 1.

Arnould, M., S. Goriely, and K. Takahashi, 2007, Physics Reports 450(4-6), 97.

Arumugam, P., L. Ferreira, and E. Maglione, 2008, Phys. Rev. C 78, 041305(R).

Arumugam, P., L. Ferreira, and E. Maglione, 2009, Phys. Lett. B 680, 443.

Arumugam, P., E. Maglione, and L. S. Ferreira, 2007, Phys. Rev. C 76(4), 044311.

Audi, G., O. Bersillon, J. Blachot, and A. H. Wapstra, 2003a, Nuclear Physics A 729(1), 3. 
Audi, G., A. H. Wapstra, and C. Thibault, 2003b, Nuclear Physics A 729(1), 337.

Auerbach, N., 1983, Physics Reports 98(5), 273.

Aumann, T., 2005, Eur. Phys. J. A26, 441.

Axelsson, L., J. Äystö, M. J. G. Borge, L. M. Fraile, H. O. U. Fynbo, A. Honkanen, P. Hornshøj, A. Jokinen, B. Jonson, P. O. Lipas, I. Martel, I. Mukha, et al., 1998, Nuclear Physics A 634(4), 475.

Azhari, A., R. A. Kryger, and M. Thoennessen, 1998, Phys. Rev. C 58, 2568.

Azuma, R. E., T. Björnstad, H. A. Gustafsson, P. G. Hansen, B. Jonson, S. Mattsson, G. Nyman, A. M. Poskanzer, and H. L. Ravn, 1980, Physics Letters B 96(1-2), 31.

Azuma, R. E., L. C. Carraz, P. G. Hansen, B. Jonson, K. L. Kratz, S. Mattsson, G. Nyman, H. Ohm, H. L. Ravn, A. Schröder, and W. Ziegert, 1979, Phys. Rev. Lett. 43(22), 1652 .

Bäck, T., B. Cederwall, K. Lagergen, R. Wyss, A. Johnson, D. Karlgren, P. Greenlees, D. Jenkins, P. Jones, D. Joss, R. Julin, S. Juutinen, et al., 2003, Eur. Phys. J. A 16, 489.

Bain, C., P. Woods, R. Coszach, T. Davinson, P. Decrock, M. Gaelens, W. Galster, M. Huyse, R. Irvine, P. Leleux, E. Lienard, M. Loiselet, et al., 1996, Physics Letters B 373(1-3), 35 .

Bambynek, W., B. Crasemann, R. W. Fink, H. U. Freund, H. Mark, C. D. Swift, R. E. Price, and P. V. Rao, 1972, Rev. Mod. Phys. 44(4), 716.

Barker, F. C., 1989, Aust. J. Phys. 42, 25.

Barker, F. C., 1999, Phys. Rev. C 59, 535.

Barker, F. C., 2001, Phys. Rev. C 63, 047303.

Barker, F. C., 2002, Phys. Rev. C 66, 047603, erratum Phys. Rev. C 67, 049902 (2003).

Barker, F. C., 2003, Phys. Rev. C 68, 054602.

Barker, F. C., and P. B. Treacy, 1962, Nuclear Physics 38, 33.

Barker, F. C., and E. K. Warburton, 1988, Nuclear Physics A $487(2), 269$.

Barmore, B., A. Kruppa, W. Nazarewicz, and T. Vertse, 2000, Phys. Rev. C 62, 054315.

Barton, R., R. McPherson, R. E. Bell, W. R. Frisken, W. T. Link, and R. B. Moore, 1963, Can. J. Phys. 41, 2007.

Batchelder, J., C. Bingham, K. Rykaczewski, K. Toth, T. Davinson, J. McKenzie, P. Woods, T. Ginter, C. Gross, J. McConnell, E. Zganjar, J. Hamilton, et al., 1998, Phys. Rev. C 57, R1042.

Batist, L., M. Górska, H. Grawe, Z. Janas, M. Kavatsyuk, M. Karny, R. Kirchner, M. La Commara, I. Mukha, A. Plochocki, and E. Roeckl, 2010, The European Physical Journal A - Hadrons and Nuclei 46, 45.

Baumann, T., A. Amthor, D. Bazin, B. Brown, C. F. III, A. Gade, T. Ginter, M. Hausmann, M. Matos, D. Morrissey, M. Portillo, A. Schiller, et al., 2007, Nature 449, 1022.

Baye, D., E. M. Tursunov, and P. Descouvemont, 2006, Phys. Rev. C 74(6), 064302.

Baz', A. I., 1967, Yad. Fiz. 5, 229.

Baz', A. I., V. I. Goldansky, V. Z. Goldberg, and Y. B. Zeldovich, 1972, Light and intermediate nuclei near the border of nuclear stability (Nauka, Moscow).

Bazin, D., F. Montes, A. Becerril, G. Lorusso, A. Amthor, T. Baumann, H. Crawford, A. Estrade, A. Gade, T. Ginter, C. J. Guess, M. Hausmann, et al., 2008, Phys. Rev. Lett. 101(25), 252501.

Becchetti, F., and G. Greenlees, 1969, Phys. Rev. 182, 1190. Becquerel, H., 1896, Comptes Rendus 122, 501.
Behrens, H., and W. Büring, 1982, Electron radial wave functions and nuclear beta-decay (Oxford University Press).

Benenson, W., and E. Kashy, 1979, Rev. Mod. Phys. 51(3), 527.

Benlliure, J., M. Fernández-Ordóñez, L. Audouin, A. Boudard, E. Casarejos, J. E. Ducret, T. Enqvist, A. Heinz, D. Henzlova, V. Henzl, A. Kelic, S. Leray, et al., 2008, Phys. Rev. C 78(5), 054605.

Benlliure, J., K. H. Schmidt, D. Cortina-Gil, T. Enqvist, F. Farget, A. Heinz, A. R. Junghans, J. Pereira, and J. Taieb, 1999, Nuclear Physics A 660(1), 87.

Benlliure, J., K. H. Schmidt, D. Cortina-Gil, T. Enqvist, F. Farget, A. Heinz, A. R. Junghans, J. Pereira, and J. Taieb, 2000, Nuclear Physics A 674(3-4), 578.

Bennaceur, K., F. Nowacki, J. Okołowicz, and M. Płoszajczak, 1998, Journal of Physics G: Nuclear and Particle Physics 24(8), 1631.

Bentley, M. A., and S. M. Lenzi, 2007, Progress in Particle and Nuclear Physics 59(2), 497.

Bergmann, U. C., L. Axelsson, M. J. G. Borge, V. N. Fedoseyev, C. Forssén, H. O. U. Fynbo, S. Grévy, P. Hornshøj, Y. Jading, B. Jonson, U. Köster, K. Markenroth, et al., 1999, Nuclear Physics A 658(2), 129.

Bernas, M., P. Armbruster, J. Benlliure, A. Boudard, E. Casarejos, S. Czajkowski, T. Enqvist, R. Legrain, S. Leray, B. Mustapha, P. Napolitani, J. Pereira, et al., 2003, Nuclear Physics A 725, 213.

Bernas, M., S. Czajkowski, P. Armbruster, H. Geissel, P. Dessagne, C. Donzaud, H.-R. Faust, E. Hanelt, A. Heinz, M. Hesse, C. Kozhuharov, C. Miehe, et al., 1994, Physics Letters B 331(1-2), 19.

Bernas, M., C. Engelmann, P. Armbruster, S. Czajkowski, F. Ameil, C. Böckstiegel, P. Dessagne, C. Donzaud, H. Geissel, A. Heinz, Z. Janas, C. Kozhuharov, et al., 1997, Physics Letters B 415(2), 111.

Bertulani, C. A., and G. Baur, 1986, Nuclear Physics A 458(4), 725.

Bethe, H., and R. Peierls, 1934, Nature 133, 532.

Bethe, H. A., 1937, Rev. Mod. Phys. 9(2), 69.

Bhattacharya, M., D. Melconian, A. Komives, S. Triambak, A. García, E. G. Adelberger, B. A. Brown, M. W. Cooper, T. Glasmacher, V. Guimaraes, P. F. Mantica, A. M. OrosPeusquens, et al., 2008, Phys. Rev. C 77(6), 065503.

Bilpuch, E. G., A. M. Lane, G. E. Mitchell, and J. D. Moses, 1976, Physics Reports 28(2), 145.

Bingham, C., J. Batchelder, K. Rykaczewski, K. Toth, C.-H. Yu, T. Ginter, C. Gross, R. Grzywacz, M. Karny, S. Kim, B. MacDonald, J. Mas, et al., 1999, Phys. Rev. C 59, R2984.

Bingham, C., M. Tantawy, J. Batchelder, M. Danchev, , T. Ginter, C. Gross, D. Fong, R. Grzywacz, K. Hagino, J. Hamilton, M. Karny, et al., 2005, Nucl. Instr. Meth. Phys. Res. B 241, 185.

Blank, B., P. Ascher, L. Audirac, G. Canchel, J. Giovinazzo, T. Kurtukian-Nieto, F. de Oliveira Santos, S. Grévy, J.-C. Thomas, C. Borcea, and L. Grigorenko, 2011, Acta Physica Polonica B 42, 545.

Blank, B., A. Bey, G. Canchel, C. Dossat, A. Fleury, J. Giovinazzo, I. Matea, N. Adimi, F. D. Oliveira, I. Stefan, G. Georgiev, S. Grevy, et al., 2005, Phys. Rev. Lett. 94, 232501.

Blank, B., and M. Borge, 2008, Progress in Particle and Nuclear Physics 60(2), 403. 
Blank, B., M. Chartier, S. Czajkowski, J. Giovinazzo, M. S. Pravikoff, J.-C. Thomas, G. de France, F. de Oliveira Santos, M. Lewitowicz, C. Borcea, R. Grzywacz, Z. Janas, et al., 2000, Phys. Rev. Lett. 84(6), 1116.

Blank, B., L. Hay, J. Huikari, S. Leblanc, S. List, J.-L. Pedroza, P. Ascher, L. Audirac, C. Borcea, G. Canchel, F. Delalee, C. Demonchy, et al., 2010, Nuclear Instruments and Methods in Physics Research Section A: Accelerators, Spectrometers, Detectors and Associated Equipment 613(1), 65.

Blank, B., and M. Płoszajczak, 2008, Rep. Prog. Phys. 71, 046301.

Blumenfeld, Y., P. Butler, J. Cornell, G. Fortuna, and M. Lindroos, 2009, Int.J.Mod.Phys. E18, 1960.

Bochkarev, O. V., A. A. Korsheninnikov, E. A. Kuz'min, I. G. Mukha, A. A. Ogloblin, L. V. Chulkov, and G. B. Yan'kov, 1984, JETP Lett. 40, 969, [Zh. Eksp. Teor. Fiz. 40 (1984) 204-207].

Bochkarev, O. V., et al., 1989, Nucl. Phys. A 505, 215.

Bogdanov, D., V. Bochin, V. Karnaukhov, and L. Petrov, 1973, Sov. J. Nucl. Phys 16, 491.

Bonetti, R., and A. Guglielmetti, 2007, Romanian Reports on Physics 59(2), 301.

Borge, M. J. G., H. Cronberg, M. Cronqvist, H. Gabelmann, P. G. Hansen, L. Johannsen, B. Jonson, S. Mattsson, G. Nyman, A. Richter, K. Riisager, O. Tengblad, et al., 1988, Nuclear Physics A 490(2), 287.

Borge, M. J. G., H. Fynbo, D. Guillemaud-Mueller, P. Hornshøj, F. Humbert, B. Jonson, T. E. Leth, G. MartínezPinedo, T. Nilsson, G. Nyman, A. Poves, I. Ramos-Lerate, et al. (the ISOLDE Collaboration), 1997, Phys. Rev. C $\mathbf{5 5}(1), \mathrm{R} 8$.

Borge, M. J. G., P. G. Hansen, B. Jonson, S. Mattsson, G. Nyman, A. Richter, and K. Riisager, 1989, Zeitschrift für Physik A Hadrons and Nuclei 332, 413.

Borzov, I., J. Cuenca-García, K. Langanke, G. MartínezPinedo, and F. Montes, 2008, Nuclear Physics A 814(1-4), 159.

Borzov, I. N., 2006, Nuclear Physics A 777, 645.

Borzov, I. N., and S. Goriely, 2000, Phys. Rev. C 62(3), 035501.

Boudard, A., J. Cugnon, S. Leray, and C. Volant, 2002, Phys. Rev. C 66(4), 044615.

Brink, D. M., M. C. Nemes, and D. Vautherin, 1983, Annals of Physics 147(1), 171.

Broda, R., 2006, Journal of Physics G: Nuclear and Particle Physics 32(6), R151.

Brown, B. A., 1990, Phys. Rev. Lett. 65(22), 2753.

Brown, B. A., 1991, Phys. Rev. C 43, R1513.

Brown, B. A., 2001, Progress in Particle and Nuclear Physics 47(2), 517.

Brown, B. A., and F. C. Barker, 2003, Phys.Rev. C 67, 041304.

Brown, B. A., F. C. Barker, and D. J. Millener, 2002, Phys. Rev. C 65, 051309.

Büscher, J., J. Ponsaers, R. Raabe, M. Huyse, P. V. Duppen, F. Aksouh, D. Smirnov, H. Fynbo, S. Hyldegaard, and C. Diget, 2008, Nuclear Instruments and Methods in Physics Research Section B: Beam Interactions with Materials and Atoms 266(19-20), 4652.

Buchmann, L., J. D'Auria, M. Dombsky, U. Giesen, K. P. Jackson, P. McNeely, J. Powell, and A. Volya, 2007, Phys. Rev. C 75(1), 012804.
Buchmann, L., G. Ruprecht, and C. Ruiz, 2009, Phys. Rev. C 80(4), 045803.

Buck, B., A. Merchant, and S. Perez, 1992, Phys. Rev. C 45, 1688.

Burbidge, E. M., G. R. Burbidge, W. A. Fowler, and F. Hoyle, 1957, Rev. Mod. Phys. 29(4), 547.

Buta, A., T. Martin, C. Timis, N. Achouri, J. C. Angélique, C. Borcea, I. Cruceru, A. Genoux-Lubain, S. Grévy, M. Lewitowicz, E. Liénard, F. M. Marqués, et al., 2000, Nuclear Instruments and Methods in Physics Research Section A: Accelerators, Spectrometers, Detectors and Associated Equipment 455(2), 412.

Caamano, M., D. Cortina-Gil, W. Mittig, H. Savajols, M. Chartier, C. Demonchy, B. Fernandez, M. G. Hornillos, A. Gillibert, B. Jurado, O. Kiselev, R. Lemmon, et al., 2008, Phys. Rev. C 78, 044001.

Cable, M. D., J. Honkanen, R. F. Parry, S. H. Zhou, Z. Y. Zhou, and J. Cerny, 1983, Phys. Rev. Lett. 50(6), 404.

del Campo, J. G., A. Galindo-Uribarri, J. R. Beene, C. J. Gross, J. F. Liang, M. L. Halbert, D. W. Stracener, D. Shapira, R. L. Varner, E. Chavez-Lomeli, and M. E. Ortiz, 2001, Phys. Rev. Lett. 86, 43.

Carpenter, M. P., R. V. F. Janssens, H. Amro, D. J. Blumenthal, L. T. Brown, D. Seweryniak, P. J. Woods, D. Ackermann, I. Ahmad, C. Davids, S. M. Fischer, G. Hackman, et al., 1997, Phys. Rev. Lett. 78(19), 3650.

Casarejos, E., J. Benlliure, J. Pereira, P. Armbruster, M. Bernas, A. Boudard, S. Czajkowski, T. Enqvist, R. Legrain, S. Leray, B. Mustapha, M. Pravikoff, et al., 2006, Phys. Rev. C 74(4), 044612.

Celardo, G. L., F. M. Izrailev, V. G. Zelevinsky, and G. P. Berman, 2008, Physics Letters B 659(1-2), 170.

Cerny, J., J. Esterl, R.A.Gough, and R.G.Sextro, 1970, Phys. Lett. 33 B, 284.

Cerny, J., D. M. Moltz, D. W. Lee, K. Peräjärvi, B. R. Barquest, L. E. Grossman, W. Jeong, and C. C. Jewett, 2009, Phys. Rev. Lett. 103(15), 152502.

Cetina, C., P. Heimberg, B. L. Berman, W. J. Briscoe, G. Feldman, L. Y. Murphy, H. Crannell, A. Longhi, D. I. Sober, J. C. Sanabria, and G. Y. Kezerashvili, 2002, Phys. Rev. C 65(4), 044622.

Chadwick, J., 1932, Nature 129, 312.

Charity, R. J., J. M. Elson, J. Manfredi, R. Shane, L. G. Sobotka, Z. Chajecki, D. Coupland, H. Iwasaki, M. Kilburn, J. Lee, W. G. Lynch, A. Sanetullaev, et al., 2010, Phys. Rev. C 82, 041304(R).

Charpak, G., W. Dominik, J. Fabre, J. Gaudaen, F. Sauli, and M. Suzuki, 1988, Nuclear Instruments and Methods in Physics Research Section A: Accelerators, Spectrometers, Detectors and Associated Equipment 269(1), 142.

Cheal, B., and K. T. Flanagan, 2010, Journal of Physics G: Nuclear and Particle Physics 37(11), 113101.

Cheikh Mhamed, M., S. Essabaa, C. Lau, M. Lebois, B. Roussičre, M. Ducourtieux, S. Franchoo, D. G. Mueller, F. Ibrahim, J. LeDu, J. Lesrel, A. Mueller, et al., 2008, Nuclear Instruments and Methods in Physics Research Section B: Beam Interactions with Materials and Atoms 266(1920), 4092.

Chow, J. C., J. D. King, N. P. T. Bateman, R. N. Boyd, L. Buchmann, J. M. D'Auria, T. Davinson, M. Dombsky, E. Gete, U. Giesen, C. Iliadis, K. P. Jackson, et al., 2002, Phys. Rev. C 66(6), 064316.

Chromik, M. J., P. G. Thirolf, M. Thoennessen, B. A. Brown, T. Davinson, D. Gassmann, P. Heckman, J. Prisciandaro, 
P. Reiter, E. Tryggestad, and P. J. Woods, 2002, Phys. Rev. C 66(2), 024313.

Cinausero, M., A. Andrighetto, L. Biasetto, L. Calabretta, J. Esposito, E. Fagottia, F. Gramegna, M. Manzolaro, P. Mastinu, A. Lombardi, A. Pisent, and G. Prete, 2009, Acta Physica Polonica B 40, 821.

Clifford, E. T. H., E. Hagberg, J. C. Hardy, H. Schmeing, R. E. Azuma, H. C. Evans, V. T. Koslowsky, U. J. Schrewe, K. S. Sharma, and I. S. Towner, 1989, Nuclear Physics A $493(2), 293$.

Cocks, J. F. C., P. A. Butler, K. J. Cann, P. T. Greenlees, G. D. Jones, J. F. Smith, P. M. Jones, R. Julin, S. Juutinen, D. Müller, M. Piiparinen, A. Savelius, et al., 2000, Journal of Physics G: Nuclear and Particle Physics 26(1), 23.

Cole, B. J., 1996, Phys. Rev. C 54, 1240.

Corradi, L., G. Pollarolo, and S. Szilner, 2009, Journal of Physics G: Nuclear and Particle Physics 36(11), 113101.

Crawford, H. L., R. V. F. Janssens, P. F. Mantica, J. S. Berryman, R. Broda, M. P. Carpenter, N. Cieplicka, B. Fornal, G. F. Grinyer, N. Hoteling, B. P. Kay, T. Lauritsen, et al., 2010, Phys. Rev. C 82(1), 014311.

Crespi, F., F. Camera, A. Bracco, B. Million, O. Wieland, V. Vandone, F. Recchia, A. Gadea, T. Kröll, D. Mengoni, E. Farnea, C. Ur, et al., 2009, Nuclear Instruments and Methods in Physics Research Section A: Accelerators, Spectrometers, Detectors and Associated Equipment 604(3), 604.

Cromaz, M., V. Riot, P. Fallon, S. Gros, B. Holmes, I. Lee, A. Macchiavelli, C. Vu, H. Yaver, and S. Zimmermann, 2008, Nuclear Instruments and Methods in Physics Research Section A: Accelerators, Spectrometers, Detectors and Associated Equipment 597(2-3), 233.

Csótó, A., 1994, Phys. Rev. C 49, 3035.

Curie, I., and F. Joliot, 1934, Comptes Rendus 198, 254.

Curie, P., and S. Curie, 1898, Comptes Rendus 127, 175.

Curie, P., S. Curie, and G. Bémont, 1898, Comptes Rendus 127, 1215.

Cuttone, G., L. Calabretta, L. Celona, F. Chines, L. Cosentino, P. Finocchiaro, A. Pappalardo, M. Re, D. Rifuggiato, and A. Rovelli, 2008, Nuclear Instruments and Methods in Physics Research Section B: Beam Interactions with Materials and Atoms 266(19-20), 4108.

Danilin, B. V., and M. V. Zhukov, 1993, Phys. At. Nucl. 56, 460.

Darby, I. G., R. K. Grzywacz, J. C. Batchelder, C. R. Bingham, L. Cartegni, C. J. Gross, M. Hjorth-Jensen, D. T. Joss, S. N. Liddick, W. Nazarewicz, S. Padgett, R. D. Page, et al., 2010, Phys. Rev. Lett. 105(16), 162502.

Datar, V. M., S. Kumar, D. R. Chakrabarty, V. Nanal, E. T. Mirgule, A. Mitra, and H. H. Oza, 2005, Phys. Rev. Lett. 94(12), 122502.

Davids, C., B. B. Back, K. Bindra, D. J. Henderson, W. Kutschera, T. Lauritsen, Y. Nagame, P. Sugathan, A. V. Ramayya, and W. B. Walters, 1992, Nuclear Instruments and Methods in Physics Research Section B: Beam Interactions with Materials and Atoms 70(1-4), 358.

Davids, C., and H. Esbensen, 2000, Phys. Rev. C 61, 054302.

Davids, C., P. Woods, J. Batchelder, C. Bingham, D. Blumenthal, L. Brown, B. Busse, L. Conticchio, T. Davinson, S. Freeman, D. Henderson, R. Irvine, et al., 1997, Phys. Rev. C 55, 2255.

Davids, C., P. Woods, D. B. J.C. Batchelder, C.R. Bingham, L. Brown, B. Busse, M. Carpenter, L. Conticchio, T. Davinson, J. DeBoer, S. Freeman, S. Hamada, D. Henderson, et al., 2001, Hyperfine Inter. 132, 133.

Davids, C., P. Woods, H. Mahmud, T. Davinson, A. Heinz, J. Ressler, K. Schmidt, D. Seweryniak, J. Shergur, A. Sonzogni, and W. Walters, 2004, Phys. Rev. C 69, 011302(R).

Davids, C., P. Woods, H. Penttilä, J. Batchelder, C. Bingham, D. Blumenthal, L. Brown, B. Busse, L. Conticchio, T. Davinson, D. Henderson, R. Irvine, et al., 1996, Phys. Rev. Lett. 76(4), 592.

Davids, C., P. Woods, D. Seweryniak, A. Sonzogni, J. Batchelder, C. Bingham, T. Davinson, D. Henderson, R. Irvine, G. Poli, J. Uusitalo, and W. Walters, 1998, Phys. Rev. Lett. 80, 1849.

Davids, C. N., 2003, Nuclear Instruments and Methods in Physics Research Section B: Beam Interactions with Materials and Atoms 204, 124.

Davids, C. N., and H. Esbensen, 2004, Phys. Rev. C 69(3), 034314.

Davinson, T., 2010, Advanced implantation detector array, http://www.ph.ed.ac.uk/ td/AIDA.

Delion, D., 2010, Theory of Particle and Cluster Emission, volume 819 of Lecture Notes in Physics (Springer Berlin/Heidelberg).

Delion, D. S., R. J. Liotta, and R. Wyss, 2006a, Phys. Rev. Lett. 96(7), 072501

Delion, D. S., R. J. Liotta, and R. Wyss, 2006b, Phys. Rep. 424(3), 113.

Descouvemont, P., E. Tursunov, and D. Baye, 2006, Nucl. Phys. A765, 370.

Diamond, W. T., 1999, Nuclear Instruments and Methods in Physics Research Section A: Accelerators, Spectrometers, Detectors and Associated Equipment 432(2-3), 471.

Diget, C. A., F. C. Barker, M. J. G. Borge, R. Boutami, P. Dendooven, T. Eronen, S. P. Fox, B. R. Fulton, H. O. U. Fynbo, J. Huikari, S. Hyldegaard, H. B. Jeppesen, et al., 2009, Phys. Rev. C 80(3), 034316.

Dobaczewski, J., I. Hamamoto, W. Nazarewicz, and J. A. Sheikh, 1994, Phys. Rev. Lett. 72(7), 981.

Dobaczewski, J., N. Michel, W. Nazarewicz, M. Płoszajczak, and J. Rotureau, 2007, Progress in Particle and Nuclear Physics 59(1), 432.

Dossat, C., N. Adimi, F. Aksouh, F. Becker, A. Bey, B. Blank, C. Borcea, R. Borcea, A. Boston, M. Caamano, G. Canchel, M. Chartier, et al., 2007, Nuclear Physics A 792(1-2), 18.

Dossat, C., A. Bey, B. Blank, G. Canchel, A. Fleury, J. Giovinazzo, I. Matea, F. de Oliveira Santos, G. Georgiev, S. Grevy, I. Stefan, J. C. Thomas, et al., 2005, Phys. Rev. C 72, 054315.

Dufour, J. P., R. D. Moral, F. Hubert, D. Jean, M. S. Pravikoff, A. Fleury, A. C. Mueller, K. H. Schmidt, K. Sümmerer, E. Hanelt, J. Frehaut, M. Beau, et al., 1988, Physics Letters B 206(2), 195.

Eichler, R., N. V. Aksenov, A. V. Belozerov, G. A. Bozhikov, V. I. Chepigin, S. N. Dmitriev, R. Dressler, H. W. Gäggeler, V. A. Gorshkov, F. Haenssler, M. G. Itkis, A. Laube, et al., 2007, Nature 447(3), 72.

Engel, G., N. Duggireddi, V. Vangapally, J. Elson, L. Sobotka, and R. Charity, 2011, Nuclear Instruments and Methods in Physics Research Section A: Accelerators, Spectrometers, Detectors and Associated Equipment In Press, Corrected Proof.

Engelmann, C., F. Ameil, P. Armbruster, M. Bernas, S. Czajkowski, P. Dessagne, C. Donzaud, H. Geissel, A. Heinz, Z. Janas, C. Kozhuharov, C. Miehé, et al., 1995, Zeitschrift für Physik A Hadrons and Nuclei 352, 351. 
Enqvist, T., J. Benlliure, F. Farget, K. H. Schmidt, P. Armbruster, M. Bernas, L. Tassan-Got, A. Boudard, R. Legrain, C. Volant, C. Böckstiegel, M. de Jong, et al., 1999, Nuclear Physics A 658(1), 47.

Eppinger, K., C. Hinke, M. Böhmer, P. Boutachkov, T. Faestermann, H. Geissel, R. Gernhäuser, M. Górska, A. Gottardo, J. Grebosz, R. Krücken, N. Kurz, et al., 2009, in GSI Scientific Report 2008, edited by K. Große (GSI Helmholtzzentrum für Schwerionenforschung GmbH), p. 147.

Esbensen, H., and C. Davids, 2001, Phys. Rev. C 63, 014315.

F. de Oliveira Santos, P. Himpe, M. Lewitowicz, I. Stefan, N. Smirnova, N. L. Achouri, J. C. Angélique, C. Angulo, L. Axelsson, D. Baiborodin, F. Becker, M. Bellegui, et al., 2005, Eur. Phys. J. A 24(2), 237.

Facina, M., C. Bachelet, M. Block, G. Bollen, D. Davies, C. Folden III, C. Guenaut, J. Huikari, E. Kwan, D. Morrissey, G. Pang, A. Prinke, et al., 2008, Nuclear Instruments and Methods in Physics Research Section B: Beam Interactions with Materials and Atoms 266(19-20), 4471.

Faesterman, T., A. Gillitzer, K. Hartel, P. Kleinle, and E. Nolte, 1984, Phys. Lett. 137B, 23.

Farnea, E., F. Recchia, D. Bazzacco, T. Kröll, Z. Podolyák, B. Quintana, and A. Gadea, 2010, Nuclear Instruments and Methods in Physics Research Section A: Accelerators, Spectrometers, Detectors and Associated Equipment 621(1-3), 331.

Fermi, E., 1934, Zeitschr. f. Phys. 88, 161.

Fernández-Domínguez, B., P. Armbruster, L. Audouin, J. Benlliure, M. Bernas, A. Boudard, E. Casarejos, S. Czajkowski, J. Ducret, T. Enqvist, B. Jurado, R. Legrain, et al., 2005, Nuclear Physics A 747(2-4), 227.

Ferreira, L., and E. Maglione, 2000, Phys. Rev. C 61, 021304(R).

Ferreira, L., and E. Maglione, 2001, Phys. Rev. Lett. 86, 1721. Ferreira, L., and E. Maglione, 2005, J. Phys. G 31, S1569.

Ferreira, L., E. Maglione, and D. Fernandes, 2002, Phys. Rev. C 65, 024323.

Ferreira, L. S., and P. Arumugan (eds.), 2007, Proton Emitting Nuclei and Related Topics, number 961 in AIP Conference Proceedings (AIP, Melville, New York).

Fiorin, G., E. Maglione, and L. S. Ferreira, 2003, Phys. Rev. C $67(5), 054302$.

Flanagan, K. T., P. Vingerhoets, M. Avgoulea, J. Billowes, M. L. Bissell, K. Blaum, B. Cheal, M. De Rydt, V. N. Fedosseev, D. H. Forest, C. Geppert, U. Köster, et al., 2009, Phys. Rev. Lett. 103(14), 142501.

Flerov, G., and K. Petrzhak, 1940, J. Phys. U.S.S.R. 3, 275.

Fomichev, A. S., I. G. Mukha, S. V. Stepantsov, L. V. Grigorenko, E. V. Litvinova, V. Chudoba, I. A. Egorova, M. S. Golovkov, A. V. Gorshkov, V. A. Gorshkov, G. Kaminski, S. A. Krupko, et al., 2011, Int. J. Mod. Phys. E20, 1491.

Fraile, L. M., and J. Äystö, 2003, Nuclear Instruments and Methods in Physics Research Section A: Accelerators, Spectrometers, Detectors and Associated Equipment 513(1-2), 287.

Fynbo, H. O. U., M. J. G. Borge, L. Axelsson, J. Äystö, U. C. Bergmann, L. M. Fraile, A. Honkanen, P. Hornsh øj, Y. Jading, A. Jokinen, B. Jonson, I. Martel, et al., 2000, Nuclear Physics A 677(1-4), 38.

Fynbo, H. O. U., M. J. G. Borge, J. Cederkäll, S. Courtin, P. Dessagne, B. Jonson, G. L. Scornet, T. Nilsson, G. Nyman, E. Poirier, K. Riisager, O. Tengblad, et al., 2004, Nuclear Physics A 736, 39.
Fynbo, H. O. U., C. A. Diget, U. C. Bergmann, M. J. G. Borge, J. Cederkäll, P. Dendooven, L. M. Fraile, S. Franchoo, V. N. Fedosseev, B. R. Fulton, W. Huang, J. Huikari, et al., 2005, Nature 433, 136.

Gaimard, J. J., and K. H. Schmidt, 1991, Nuclear Physics A 531(3-4), 709.

Galitsky, V. M., and V. F. Cheltsov, 1964, Nucl. Phys. 56, 86.

Gamow, G., 1928, Zeitschr. f. Phys. 51, 204.

Garrido, E., D. V. Fedorov, H. O. U. Fynbo, and A. S. Jensen, 2007, Physics Letters B 648(4), 274.

Garrido, E., A. Jensen, and D. Fedorov, 2008, Phys. Rev. C 78, 034004 .

Gavron, A., 1980, Phys. Rev. C 21(1), 230.

Geesaman, D. F., R. L. McGrath, P. M. S. Lesser, P. P. Urone, and B. VerWest, 1977, Phys. Rev. C 15, 1835.

Geiger, H., and J. Nutall, 1912, Phil. Mag. 23, 439.

Geissel, H., P. Armbruster, K. H. Behr, A. Brünle, K. Burkard, M. Chen, H. Folger, B. Franczak, H. Keller, O. Klepper, B. Langenbeck, F. Nickel, et al., 1992, Nuclear Instruments and Methods in Physics Research Section B: Beam Interactions with Materials and Atoms 70(1-4), 286.

Geissel, H., G. Münzenberg, and K. Riisager, 1995, Annual Review of Nuclear and Particle Science 45(1), 163.

Gelletly, W., and J. Eberth, 2006, in The Euroschool Lectures on Physics With Exotic Beams, Vol.II, Lect. Notes Phys. 700, edited by J. Al-Khalili and E. Roeckl (Springer, Berlin Heidelberg), p. 79.

Gillitzer, A., T. Faesterman, K. Hartel, P. Kleinle, and E. Nolte, 1987, Z. Phys. A 326, 107.

Ginter, T., J. Batchelder, C. Bingham, C. Gross, R. Grzywacz, J. Hamilton, Z. Janas, M. Karny, S. Kim, J. Mas, J. McConnel, A. Piechaczek, et al., 2000, Phys. Rev. C 61, 014308.

Ginter, T., J. Batchelder, C. Bingham, C. Gross, R. Grzywacz, J. Hamilton, Z. Janas, M. Karny, A. Piechaczek, A. Ramayya, K. Rykaczewski, W. Walters, et al., 2003, Phys. Rev. C 68, 032330.

Giovinazzo, J., B. Blank, C. Borcea, G. Canchel, J. C. Dalouzy, C. E. Demonchy, F. de Oliveira Santos, C. Dossat, S. Grevy, L. Hay, J. Huikari, S. Leblanc, et al., 2007, Phys. Rev. Lett. 99, 102501.

Giovinazzo, J., B. Blank, M. Chartier, S. Czajkowski, A. Fleury, M. J. Lopez Jimenez, M. S. Pravikoff, J.-C. Thomas, F. de Oliveira Santos, M. Lewitowicz, V. Maslov, M. Stanoiu, et al., 2002, Phys. Rev. Lett. 89(10), 102501.

Giovinazzo, J., P. Dessagne, and C. Miehé, 2000, Nuclear Physics A 674(3-4), 394.

Goldansky, V., 1960, Nucl. Phys. 19, 482.

Goldansky, V. I., 1961, Nucl. Phys. 27, 648.

Golovkov, M. S., L. V. Grigorenko, A. S. Fomichev, S. A. Krupko, Y. T. Oganessian, A. M. Rodin, S. I. Sidorchuk, R. S. Slepnev, S. V. Stepantsov, G. M. Ter-Akopian, R. Wolski, M. G. Itkis, et al., 2005, Phys. Rev. C 72, 064612 .

Golovkov, M. S., L. V. Grigorenko, A. S. Fomichev, Y. T. Oganessian, Y. I. Orlov, A. M. Rodin, S. I. Sidorchuk, R. S. Slepnev, S. V. Stepantsov, G. M. Ter-Akopian, and R. Wolski, 2004, Phys. Lett. B 588, 163.

Golovkov, M. S., L. V. Grigorenko, G. M. Ter-Akopian, A. S. Fomichev, Y. T. Oganessian, V. A. Gorshkov, S. A. Krupko, A. M. Rodin, S. I. Sidorchuk, R. S. Slepnev, S. V. Stepantsov, R. Wolski, et al., 2009, Phys. Lett. B 672, 22. 
Goriely, S., N. Chamel, and J. M. Pearson, 2009, Phys. Rev. Lett. 102(15), 152503.

Goriely, S., N. Chamel, and J. M. Pearson, 2010, Phys. Rev. C 82(3), 035804.

Grawe, H., K. Langanke, and G. Martínez-Pinedo, 2007, Reports on Progress in Physics 70(9), 1525.

Grévy, S., J. C. Angélique, P. Baumann, C. Borcea, A. Buta, G. Canchel, W. N. Catford, S. Courtin, J. M. Daugas, F. de Oliveira, P. Dessagne, Z. Dlouhy, et al., 2004, Physics Letters B 594(3-4), 252.

Grigorenko, L. V., 2009, Physics of Particles and Nuclei 40, 674.

Grigorenko, L. V., I. A. Egorova, M. V. Zhukov, R. J. Charity, and K. Miernik, 2010, Phys. Rev. C 82, 014615, 12 pages.

Grigorenko, L. V., R. C. Johnson, I. G. Mukha, I. J. Thompson, and M. V. Zhukov, 2000, Phys. Rev. Lett. 85, 22.

Grigorenko, L. V., I. G. Mukha, I. J. Thompson, and M. V. Zhukov, 2002, Phys. Rev. Lett. 88, 042502.

Grigorenko, L. V., I. G. Mukha, and M. V. Zhukov, 2003a, Nucl. Phys. A 713, 372, erratum Nucl. Phys. A740 (2004) 401.

Grigorenko, L. V., T. D. Wiser, K. Mercurio, R. J. Charity, R. Shane, L. G. Sobotka, J. M. Elson, A. H. Wuosmaa, A. Banu, M. McCleskey, L. Trache, R. E. Tribble, et al., 2009a, Phys. Rev. C 80, 034602.

Grigorenko, L. V., T. D. Wiser, K. Miernik, R. J. Charity, M. Pfützner, A. Banu, C. R. Bingham, M. Ćwiok, I. G. Darby, W. Dominik, J. M. Elson, T. Ginter, et al., 2009b, Phys. Lett. B 677, 30.

Grigorenko, L. V., and M. V. Zhukov, 2007a, Phys. Rev. C 76, 014008.

Grigorenko, L. V., and M. V. Zhukov, 2007b, Phys. Rev. C 76, 014009.

Grigorenko, L. V., and M. V. Zhukov, 2008, Phys. Rev. C 77, 034611.

Grigorenko, L. V., and M. V. Zhukov, 2003b, Phys. Rev. C 68, 054005.

Grigorenko, L. V., I. G. Mukha, C. Scheidenberger, and M. V. Zhukov, 2011, Phys. Rev. C 84, 021303(R).

Grinyer, G. F., C. E. Svensson, and B. A. Brown, 2010, Nuclear Instruments and Methods in Physics Research Section A: Accelerators, Spectrometers, Detectors and Associated Equipment 622(1), 236.

Gross, C. J., T. N. Ginter, D. Shapira, W. T. Milner, J. W. McConnell, A. N. James, J. W. Johnson, J. Mas, P. F. Mantica, R. L. Auble, J. J. Das, J. L. Blankenship, et al., 2000, Nuclear Instruments and Methods in Physics Research Section A: Accelerators, Spectrometers, Detectors and Associated Equipment 450(1), 12.

Grotz, K., and H. V. Klapdor, 1990, The weak interaction in nuclear, particle and astrophysics (Adam Hilger).

Grzywacz, R., 2003, Nuclear Instruments and Methods in Physics Research Section B: Beam Interactions with Materials and Atoms 204, 649.

Grzywacz, R., R. Beraud, C. Borcea, A. Emsallem, M. Glogowski, H. Grawe, D. Guillemaud-Mueller, M. HjorthJensen, M. Houry, M. Lewitowicz, A. C. Mueller, A. Nowak, et al., 1998, Phys.Rev.Lett. 81, 766.

Grzywacz, R., M. Karny, K. Rykaczewski, J. Batchelder, C. Bingham, D. Fong, C. Gross, W. Królas, C. Mazzocchi, A. Piechaczek, M. Tantawy, J. Winger, et al., 2005, Eur. Phys. J. A 25, 145.

Gurney, R., and E. Condon, 1928, Nature 122, 439.
Gurvitz, S. A., and G. Kalbermann, 1987, Phys. Rev. Lett. $\mathbf{5 9}(3), 262$.

Hagino, K., 2001, Phys. Rev. C 64, 04104(R).

Hall, H. L., and D. C. Hoffman, 1992, Annual Review of Nuclear and Particle Science 42(1), 147.

Hamamoto, I., and H. Sagawa, 1993, Phys. Rev. C 48(3), R960.

Hansen, P. G., and B. Jonson, 1988, in Particle Emission from Nuclei, edited by D. Poenaru and M. Ivascu (CRC), volume III, p. 157.

Hansen, P. G., B. Jonson, and A. Richter, 1990, Nuclear Physics A 518(1-2), 13.

Hardy, J. C., and E. Hagberg, 1988, in Particle Emission from Nuclei, edited by D. Poenaru and M. Ivascu (CRC), volume III, p. 99.

Hardy, J. C., B. Jonson, and P. G. Hansen, 1978, Nuclear Physics A 305(1), 15.

Harney, H. L., A. Richter, and H. A. Weidenmüller, 1986, Rev. Mod. Phys. 58(3), 607.

Heine, A., T. Faesterman, A. Gillitzer, J. Hamolka, M. Kopf, and W. Wagner, 1991, Z. Phys. A 340, 225.

Henzlova, D., K.-H. Schmidt, M. V. Ricciardi, A. Kelić, V. Henzl, P. Napolitani, L. Audouin, J. Benlliure, A. Boudard, E. Casarejos, J. E. Ducret, T. Enqvist, et al., 2008, Phys. Rev. C 78(4), 044616.

Hirayama, Y., T. Shimoda, H. Izumi, A. Hatakeyama, K. P. Jackson, C. D. P. Levy, H. Miyatake, M. Yagi, and H. Yano, 2005, Physics Letters B 611(3-4), 239.

Hofmann, S., 1995, Radiochim. Acta 70/71, 93.

Hofmann, S., 1996, Nuclear Decay Modes. (Institute of Physics Publishing), chapter 3.Proton Radioactivity, pp. 143-203.

Hofmann, S., 2009a, in The Euroschool Lectures on Physics with Exotic Beams, Vol. III, Lect. Notes Phys. 764, edited by J. Al-Khalili and E. Roeckl (Springer, Berlin Heidelberg), p. 203.

Hofmann, S., 2009b, Russian Chemical Reviews 78(12), 1123.

Hofmann, S., and G. Münzenberg, 2000, Rev. Mod. Phys. $\mathbf{7 2}(3), 733$.

Hofmann, S., W. Reisdorf, G. Münzenberg, F. P. Hessberger, J. R. H. Schneider, and P. Armbruster, 1982, Z. Phys. A305, 111.

Holstein, B. R., 1974, Rev. Mod. Phys. 46(4), 789.

Homma, H., E. Bender, M. Hirsch, K. Muto, H. V. KlapdorKleingrothaus, and T. Oda, 1996, Phys. Rev. C 54(6), 2972.

Hosmer, P., H. Schatz, A. Aprahamian, O. Arndt, R. R. C. Clement, A. Estrade, K. Farouqi, K.-L. Kratz, S. N. Liddick, A. F. Lisetskiy, P. F. Mantica, P. Möller, et al., 2010, Phys. Rev. C 82(2), 025806.

Hosmer, P. T., H. Schatz, A. Aprahamian, O. Arndt, R. R. C. Clement, A. Estrade, K. L. Kratz, S. N. Liddick, P. F. Mantica, W. F. Mueller, F. Montes, A. C. Morton, et al., 2005, Phys.Rev.Lett. 94, 112501.

Huang, K.-N., M. Auyangi, M. Chen, B. Crasemann, and H. Mark, 1976, Atom. Data Nucl. Data Tabl. 18, 243.

Hubbard-Nelson, B., M. Momayezi, and W. K. Warburton, 1999, Nuclear Instruments and Methods in Physics Research Section A: Accelerators, Spectrometers, Detectors and Associated Equipment 422(1-3), 411.

Hyldegaard, S., 2010, Beta-decay studies of ${ }^{8} B e$ and ${ }^{12} C$, Ph.D. thesis, Aarhus University.

Hyldegaard, S., M. Alcorta, B. Bastin, M. J. G. Borge, R. Boutami, S. Brandenburg, J. Büscher, P. Dendooven, 
C. A. Diget, P. Van Duppen, T. Eronen, S. P. Fox, et al., 2010, Phys. Rev. C 81(2), 024303.

Hyldegaard, S., C. Forssén, C. A. Diget, M. Alcorta, F. C. Barker, B. Bastin, M. J. G. Borge, R. Boutami, S. Brandenburg, J. Büscher, P. Dendooven, P. V. Duppen, et al., 2009, Physics Letters B 678(5), 459.

Ichimura, M., H. Sakai, and T. Wakasa, 2006, Progress in Particle and Nuclear Physics 56(2), 446.

Irvine, R., C. Davids, P. Woods, D. Blumenthal, L. Brown, L. Conticchio, T. Davinson, D. Henderson, J. Mackenzie, H. Penttilä, D. Seweryniak, and W. Walters, 1997, Phys. Rev. C 55, R1621.

Jackson, K., C. Cardinal, H. Evans, N. Jelley, and J. Cerny, 1970, Phys. Lett. 33 B, 281.

Janas, Z., L. Batist, J. Döring, M. Gierlik, R. Kirchner, J. Kurcewicz, H. Mahmud, C. Mazzocchi, A. Plochocki, E. Roeckl, K. Schmidt, P. J. Woods, et al., 2005, The European Physical Journal A - Hadrons and Nuclei 23, 401.

Jänecke, J., 1965, Nucl. Phys. 61, 326.

Jenkins, D. G., M. Muikku, P. T. Greenlees, K. Hauschild, K. Helariutta, P. M. Jones, R. Julin, S. Juutinen, H. Kankaanpää, N. S. Kelsall, H. Kettunen, P. Kuusiniemi, et al., 2000, Phys. Rev. C 62(2), 021302.

Jensen, A. S., K. Riisager, D. V. Fedorov, and E. Garrido, 2004, Rev. Mod. Phys. 76(1), 215.

Jeppesen, H., U. C. Bergmann, M. J. G. Borge, J. Cederkäll, V. N. Fedoseyev, H. O. U. Fynbo, V. Y. Hansper, B. Jonson, K. Markenroth, V. I. Mishin, T. Nilsson, G. Nyman, et al., 2002, Nuclear Physics A 709(1-4), 119.

Johansson, H., Y. Aksyutina, T. Aumann, K. Boretzky, M. Borge, A. Chatillon, L. Chulkov, D. Cortina-Gil, U. D. Pramanik, H. Emling, C. Forssen, H. Fynbo, et al., 2010, Nucl. Phys. A847, 66.

Jonson, B., and G. Nyman, 1996, in Nuclear Decay Modes, edited by D. Poenaru (IOP publishing), p. 102.

Jonson, B., and K. Riisager, 2001, Nuclear Physics A 693(12), 77 .

Joss, D. T., I. G. Darby, R. D. Page, J. Uusitalo, S. Eeckhaudt, T. Grahn, P. T. Greenlees, P. M. Jones, R. Julin, S. Juutinen, S. Ketelhut, M. Leino, et al., 2006, Phys. Lett. B 641, 34.

JPG, 2011, Focus section: "physics with reaccelerated isol beams", Journal of Physics G: Nuclear and particle Physics, Volume 38, Number 2.

Julin, R., 2010, Nucl. Phys. A 834, 15c.

Kadmensky, S., and A. Sonzogni, 2000, Phys. Rev. C 62, 054601.

Kanada-En'yo, Y., 2010, Phys. Rev. C 81(3), 034321.

Kankainen, A., V.-V. Elomaa, L. Batist, S. Eliseev, T. Eronen, U. Hager, J. Hakala, A. Jokinen, I. D. Moore, Y. N. Novikov, H. Penttilä, A. Popov, et al., 2008, Phys. Rev. Lett. 101, 142503.

Karnaukhov, V., G. Ter-Akopian, and V. Subbotin, 1963, in Proc. Asilomar Conf. on Reactions Between Complex Nuclei, edited by e. a. A. Ghiorso (University of California Press), p. 434.

Karny, M., R. K. Grzywacz, J. C. Batchelder, C. R. Bingham, C. J. Gross, K. Hagino, J. H. Hamilton, Z. Janas, W. D. Kulp, J. W. McConnell, M. Momayezi, A. Piechaczek, et al., 2003, Phys. Rev. Lett. 90(1), 012502.

Karny, M., K. Rykaczewski, R. Grzywacz, J. Batchelder, C. Bingham, C. Goodin, C. Gross, J. Hamilton, A. Korgul, W. Królas, S. Liddick, K. Li, et al., 2008, Phys. Lett. B 664, 52 .
Kaufmann, W., 1902, Phys. Zeitschr. 4, 54.

KeKelis, G. J., M. S. Zisman, D. K. Scott, R. Jahn, D. J. Vieira, J. Cerny, and F. Ajzenberg-Selove, 1978, Phys. Rev. C 17, 1929.

Kelić, A., M. Ricciardi, and K.-H. Schmidt, 2008, in Proceedings of the Joint ICTP-IAEA Advanced Workshop on Model Codes for Spallation Reactions, edited by D. Filges, S. Leray, Y. Yariv, A. Mengoni, A. Stanculescu, and G. Mank (IAEA INDC(NDS)-530, Vienna), pp. $181-221$.

Kettunen, H., T. Enqvist, T. Grahn, P. Greenlees, P. Jones, R. Julin, S. Juutinen, A. Keenan, P. Kuusiniemi, M. Leino, A.-P.Leppänen, P. Nieminen, et al., 2004, Phys. Rev. C 69, 054323.

Kettunen, H., P. Greenlees, K. Helariutta, P. Jones, R. Julin, S. Juutinen, P. Kuusiniemi, M. Leino, M. Muikku, P. Nieminen, and J. Uusitalo, 2001, Acta. Phys. Pol. B 32, 989.

Kirsebom, O. S., 2010, ${ }^{8} B$ Neutrinos and ${ }^{12} C$ Resonances, Ph.D. thesis, Aarhus University.

Kirsebom, O. S., M. Alcorta, M. J. G. Borge, M. Cubero, C. A. Diget, R. Dominguez-Reyes, L. Fraile, B. R. Fulton, H. Fynbo, D. Galaviz, G. Garcia, S. Hyldegaard, et al., 2009, Physics Letters B 680(1), 44.

Klepper, O., T. Batsch, S. Hofmann, R. Kirchner, W. Kurcewicz, W. Reisdorf, E. Roeckl, D. Schardt, and G. Nyman, 1982, Z. Phys. A305, 125.

Kluge, H.-J., F. Herfurth, O. Kester, W. Nörtershäuser, and W. Quint, 2008, Nuclear Instruments and Methods in Physics Research Section B: Beam Interactions with Materials and Atoms 266(19-20), 4542.

Korgul, A., K. P. Rykaczewski, C. J. Gross, R. K. Grzywacz, S. N. Liddick, C. Mazzocchi, J. C. Batchelder, C. R. Bingham, I. G. Darby, C. Goodin, J. H. Hamilton, J. K. Hwang, et al., 2008, Phys. Rev. C 77(3), 034301.

Korsheninnikov, A., E. Nikolskii, E. Kuzmin, A. Ozawa, K. Morimoto, F. Tokanai, R. Kanungo, I. Tanihata, N. Timofeyuk, M. Golovkov, A. Fomichev, A. Rodin, et al., 2003, Phys. Rev. Lett. 90, 082501.

Korsheninnikov, A., K. Yoshida, D. Aleksandrov, N. Aoi, Y. Doki, N. Inabe, M. Fujimaki, T. Kobayashi, H. Kumagai, C.-B. Moon, E. Nikolsky, M. Obuti, et al., 1994, Phys. Lett. B326, 31.

Kox, S., A. Gamp, C. Perrin, J. Arvieux, R. Bertholet, J. F. Bruandet, M. Buenerd, R. Cherkaoui, A. J. Cole, Y. ElMasri, N. Longequeue, J. Menet, et al., 1987, Phys. Rev. C 35(5), 1678.

Kratz, K.-L., K. Farouqi, and B. Pfeiffer, 2007, Progress in Particle and Nuclear Physics 59(1), 147.

Kruppa, A., B. Barmore, W. Nazarewicz, and T. Vertse, 2000, Phys. Rev. Lett. 84, 4549.

Kruppa, A. T., and W. Nazarewicz, 2004, Phys. Rev. C 69(5), 054311.

Kryger, R. A., A. Azhari, M. Hellström, J. H. Kelley, T. Kubo, R. Pfaff, E. Ramakrishnan, B. M. Sherrill, M. Thoennessen, S. Yokoyama, R. J. Charity, J. Dempsey, et al., 1995, Phys. Rev. Lett. 74, 860.

Kudryavtsev, Y., T. Cocolios, J. Gentens, O. Ivanov, M. Huyse, D. Pauwels, M. Sawicka, T. Sonoda, P. V. den Bergh, and P. V. Duppen, 2008, Nuclear Instruments and Methods in Physics Research Section B: Beam Interactions with Materials and Atoms 266(19-20), 4368.

Kumar, R., F. Molina, S. Pietri, E. Casarejos, A. Algora, J. Benlliure, P. Doornenbal, J. Gerl, M. Górska, I. Kojouharov, Z. Podolyák, W. Prokopowicz, et al., 2009, Nuclear Instruments and Methods in Physics Research Section 
A: Accelerators, Spectrometers, Detectors and Associated Equipment 598(3), 754.

Kurtukian-Nieto, T., J. Benlliure, and K.-H. Schmidt, 2008, Nuclear Instruments and Methods in Physics Research Section A: Accelerators, Spectrometers, Detectors and Associated Equipment 589(3), 472.

Kuznetsov, V., and N. Skobelev, 1999, Physics of Particles and Nuclei 30(6), 666.

Lagergren, K., D. Joss, R. Wyss, B. Cederwall, C. Barton, S. Eeckhaudt, T. Grahn, P. Greenlees, B. Hadinia, P. Jones, R. Julin, S. Juutinen, et al., 2006, Phys. Rev. C 74, 024316.

Lane, A. M., and R. G. Thomas, 1958, Rev. Mod. Phys. 30, 257.

Langanke, K., and G. Martínez-Pinedo, 2000, Nuclear Physics A 673(1-4), 481.

Langanke, K., and G. Martínez-Pinedo, 2003, Rev. Mod. Phys. 75(3), 819.

Larsson, P. O., T. Batsch, R. Kirchner, O. Klepper, W. Kurcewicz, E. Roeckl, D. Schardt, W. F. Feix, G. Nyman, and P. Tidemand-Petersson, 1983, Z.Phys. A314, 9.

Le Gentil, E., T. Aumann, C. O. Bacri, J. Benlliure, S. Bianchin, M. Böhmer, A. Boudard, J. Brzychczyk, E. Casarejos, M. Combet, L. Donadille, J. E. Ducret, et al., 2008, Phys. Rev. Lett. 100(2), 022701.

Lecesne, N., 2008, Nuclear Instruments and Methods in Physics Research Section B: Beam Interactions with Materials and Atoms 266(19-20), 4338.

Lee, I. Y., M. A. Deleplanque, and K. Vetter, 2003, Reports on Progress in Physics 66(7), 1095.

Leino, M., J. Äystö, T. Enqvist, P. Heikkinen, A. Jokinen, M. Nurmia, A. Ostrowski, W. H. Trzaska, J. Uusitalo, K. Eskola, P. Armbruster, and V. Ninov, 1995, Nuclear Instruments and Methods in Physics Research Section B: Beam Interactions with Materials and Atoms 99(1-4), 653.

Lewitowicz, M., 2008, Nuclear Physics A 805(1-4), 519c.

Lewitowicz, M., R. Anne, G. Auger, D. Bazin, C. Borcea, V. Borrel, J. Corre, T. Dörfler, A. Fomichov, R. Grzywacz, D. Guillemaud-Mueller, R. Hue, et al., 1994, Physics Letters B 332(1-2), 20.

Li, Z. H., J. L. Lou, Y. L. Ye, H. Hua, D. X. Jiang, X. Q. Li, S. Q. Zhang, T. Zheng, Y. C. Ge, Z. Kong, L. H. Lv, C. Li, et al., 2009, Phys. Rev. C 80(5), 054315.

Liddick, S. N., R. Grzywacz, C. Mazzocchi, R. D. Page, K. P. Rykaczewski, J. C. Batchelder, C. R. Bingham, I. G. Darby, G. Drafta, C. Goodin, C. J. Gross, J. H. Hamilton, et al., 2006, Phys. Rev. Lett. 97(8), 082501.

Litvinov, Y. A., and F. Bosch, 2011, Reports on Progress in Physics 74(1), 016301.

Litvinova, E., P. Ring, and V. Tselyaev, 2008, Phys. Rev. C 78, 014312.

Livingston, K., P. Woods, T. Davinson, N. Davis, S. Hofmann, A. James, R. Page, P. Selin, and A. Shotter, 1993, Phys. Lett. B 312, 46.

Lunney, D., J. M. Pearson, and C. Thibault, 2003, Rev. Mod. Phys. 75(3), 1021.

Macfarlane, R. D., and A. Siivola, 1965, Phys. Rev. Lett. 14(4), 114.

Madurga, M., M. J. G. Borge, M. Alcorta, L. M. Fraile, H. O. U. Fynbo, B. Jonson, O. Kirsebom, T. Nilsson, G. Nyman, A. Perea, K. Riisager, O. Tengblad, et al., 2009, The European Physical Journal A - Hadrons and Nuclei 42, 415.

Madurga, M., M. J. G. Borge, J. C. Angelique, L. Bao, U. Bergmann, A. Buta, J. Cederkäll, C. A. Diget, L. M.
Fraile, H. O. U. Fynbo, H. B. Jeppesen, B. Jonson, et al., 2008, Nuclear Physics A 810(1-4), 1.

Magill, J., G. Pfennig, and J. Galy, 2009, Karlsruher Nuklidkarte (European Communities 2006-2009), 7th edition, revised printing 2009 edition.

Maglione, E., and L. Ferreira, 2000, Phys. Reev. C 61, 047307.

Maglione, E., and L. Ferreira, 2002, Eur. Phys. J. A 15, 89.

Maglione, E., L. Ferreira, and R. Liotta, 1998, Phys. Rev. Lett. 81, 538 .

Maglione, E., S. Ferreira, and R. Liotta, 1999, Phys. Rev. C 59, R589.

Mahmud, H., C. Davids, P. Woods, T. Davidson, A. Heinz, J. Ressler, K. Schmidt, D. Seweryniak, J. Shergur, A. Sonsogni, and W. Walters, 2002, Eur. Phys. J. A 15, 85.

Mahmud, H., C. Davids, P. Woods, T. Davinson, A. Heinz, G. Poli, J. Ressler, K. Schmidt, D. Seweryniak, M. Smith, A. Sonzodni, J. Uusitalo, et al., 2001, Phys. Rev. C 64, 031303.

Martínez-Pinedo, G., D. Mocelj, N. T. Zinner, A. Kelic, K. Langanke, I. Panov, B. Pfeiffer, T. Rauscher, K.-H. Schmidt, and F.-K. Thielemann, 2007, Progress in Particle and Nuclear Physics 59(1), 199.

Mattoon, C. M., F. Sarazin, C. Andreoiu, A. N. Andreyev, R. A. E. Austin, G. C. Ball, R. S. Chakrawarthy, D. Cross, E. S. Cunningham, J. Daoud, P. E. Garrett, G. F. Grinyer, et al., 2009, Phys. Rev. C 80(3), 034318.

Mazzocchi, C., R. Grzywacz, S. Liddick, K. Rykaczewski, H. Schatz, J. Batchelder, C. Bingham, C. Gross, J. Hamilton, J. Hwang, S. Ilyushkin, A. Korgul, et al., 2007, Phys. Rev. Lett. 98, 212501.

Mehren, T., B. Pfeiffer, S. Schoedder, K.-L. Kratz, M. Huhta, P. Dendooven, A. Honkanen, G. Lhersonneau, M. Oinonen, J.-M. Parmonen, H. Penttilä, A. Popov, et al., 1996, Phys. Rev. Lett. 77(3), 458.

Mianowski, S., H. Czyrkowski, R. Dąbrowski, W. Dominik, Z. Janas, K. Miernik, M. Pfützner, A. S. Fomichev, M. S. Golovkov, L. V. Grigorenko, S. A. Krupko, S. I. Sidorchuk, et al., 2010, Acta Physica Polonica B 41, 449.

Michel, N., W. Nazarewicz, J. Okołowicz, and M. Płoszajczak, 2010a, Journal of Physics G: Nuclear and Particle Physics 37(6), 064042.

Michel, N., W. Nazarewicz, and M. Płoszajczak, 2010b, Phys. Rev. C 82(4), 044315.

Michel, N., W. Nazarewicz, M. Płoszajczak, and K. Bennaceur, 2002, Phys. Rev. Lett. 89(4), 042502.

Michel, N., W. Nazarewicz, M. Płoszajczak, and J. Okołowicz, 2003, Phys. Rev. C 67, 054311.

Michel, N., W. Nazarewicz, M. Płoszajczak, and T. Vertse, 2009, Journal of Physics G: Nuclear and Particle Physics 36(1), 013101.

Miernik, K., W. Dominik, H. Czyrkowski, R. Dąbrowski, A. Fomitchev, M. Golovkov, Z. Janas, W. Kuśmierz, M. Pfützner, A. Rodin, S. Stepantsov, R. Slepniev, et al., 2007a, Nuclear Instruments and Methods in Physics Research Section A: Accelerators, Spectrometers, Detectors and Associated Equipment 581(1-2), 194.

Miernik, K., W. Dominik, Z. Janas, M. Pfützner, C. R. Bingham, H. Czyrkowski, M. Ćwiok, I. G. Darby, R. Dąbrowski, T. Ginter, R. Grzywacz, M. Karny, et al., 2007b, Phys. Rev. C 76(4), 041304.

Miernik, K., W. Dominik, Z. Janas, M. Pfützner, L. Grigorenko, C. R. Bingham, H. Czyrkowski, M. Ćwiok, I. G. Darby, R. Dąbrowski, T. Ginter, R. Grzywacz, et al., 2007c, 
Phys. Rev. Lett. 99, 192501.

Miernik, K., W. Dominik, Z. Janas, M. Pfützner, L. V. Grigorenko, C. Bingham, H. Czyrkowski, M. Ćwiok, I. G. Darby, R. Dąbrowski, T. Ginter, R. Grzywacz, et al., 2009, Eur. Phys. J. A 42, 431.

Millener, D., 2005, The European Physical Journal A Hadrons and Nuclei 25, 97.

Mitchell, G. E., A. Richter, and H. A. Weidenmüller, 2010, Rev. Mod. Phys. 82(4), 2845.

Möller, P., J. R. Nix, W. D. Myers, and W. J. Swištecki, 1995, Atomic Data and Nuclear Data Tables 59(2), 185.

Münzenberg, G., W. Faust, S. Hofmann, P. Armbruster, K. Güttner, and H. Ewald, 1979, Nucl. Inst. Meth. 161, 65.

Mohr, P., 2007, Eur. Phys. J. A 31, 23.

Möller, P., J. R. Nix, and K.-L. Kratz, 1997, Atomic Data and Nuclear Data Tables 66(2), 131.

Möller, P., B. Pfeiffer, and K.-L. Kratz, 2003, Phys. Rev. C 67(5), 055802.

Möller, P., A. J. Sierk, T. Ichikawa, A. Iwamoto, R. Bengtsson, H. Uhrenholt, and S. Åberg, 2009, Phys. Rev. C 79(6), 064304.

Moltz, D., J. Robertson, J. Batchelder, and J. Cerny, 1994, Nuclear Instruments and Methods in Physics Research Section A: Accelerators, Spectrometers, Detectors and Associated Equipment 349(1), 210.

Montanari, D., S. Leoni, D. Mengoni, G. Benzoni, N. Blasi, G. Bocchi, P. Bortignon, A. Bracco, F. Camera, G. Colo, A. Corsi, F. Crespi, et al., 2011, Physics Letters B 697(4), 288.

Morrissey, D. J., B. M. Sherrill, M. Steiner, A. Stolz, and I. Wiedenhoever, 2003, Nuclear Instruments and Methods in Physics Research Section B: Beam Interactions with Materials and Atoms 204, 90.

Mueller, A. C., and R. Anne, 1991, Nuclear Instruments and Methods in Physics Research Section B: Beam Interactions with Materials and Atoms 56-57(Part 1), 559.

Mukha, I., H. Grawe, E. Roeckl, and S. Tabor, 2008a, Phys. Rev. C 78, 039803.

Mukha, I., L. Grigorenko, K. Sümmerer, L. Acosta, M. A. G. Alvarez, E. Casarejos, A. Chatillon, D. Cortina-Gil, J. Espino, A. Fomichev, J. E. Garcia-Ramos, H. Geissel, et al., 2008b, Phys. Rev. C 77, 061303(R).

Mukha, I., E. Roeckl, L. Batist, A. Blazhev, J. Döring, H. Grawe, L. Grigorenko, M. Huyse, Z. Janas, R. Kirchner, M. L. Commara, C. Mazzocchi, et al., 2006, Nature 439, 298 .

Mukha, I., E. Roeckl, J. Döring, L. Batist, A. Blazhev, H. Grawe, C. R. Hoffman, M. Huyse, Z. Janas, R. Kirchner, M. La Commara, C. Mazzocchi, et al., 2005, Phys. Rev. Lett. 95(2), 022501.

Mukha, I., K. Sümmerer, L. Acosta, M. A. G. Alvarez, E. Casarejos, A. Chatillon, D. Cortina-Gil, I. A. Egorova, J. M. Espino, A. Fomichev, J. E. García-Ramos, H. Geissel, et al., 2010, Phys. Rev. C 82(5), 054315.

Mukha, I., K. Sümmerer, L. Acosta, M. A. G. Alvarez, E. Casarejos, A. Chatillon, D. Cortina-Gil, J. Espino, A. Fomichev, J. E. Garcia-Ramos, H. Geissel, J. GomezCamacho, et al., 2007, Phys. Rev. Lett. 99, 182501.

Mukha, I., N. K. Timofeyuk, K. Sümmerer, L. Acosta, M. A. G. Alvarez, E. Casarejos, A. Chatillon, D. CortinaGil, J. M. Espino, A. Fomichev, J. E. Garcia-Ramos, H. Geissel, et al., 2009, Phys. Rev. C 79, 061301(R).
Nakata, H., T. Tachibana, and M. Yamada, 1997, Nuclear Physics A 625(3), 521.

Napolitani, P., K.-H. Schmidt, L. Tassan-Got, P. Armbruster, T. Enqvist, A. Heinz, V. Henzl, D. Henzlova, A. Kelić, R. Pleskač, M. V. Ricciardi, C. Schmitt, et al., 2007, Phys. Rev. C 76(6), 064609.

Navrátil, P., V. G. Gueorguiev, J. P. Vary, W. E. Ormand, and A. Nogga, 2007, Phys. Rev. Lett. 99(4), 042501.

Nazarewicz, W., J. Dobaczewski, T. R. Werner, J. A. Maruhn, P.-G. Reinhard, K. Rutz, C. R. Chinn, A. S. Umar, and M. R. Strayer, 1996, Phys. Rev. C 53(2), 740.

Nesterov, A., F. Arickx, J. Broeckhove, and V. Vasilevsky, 2010, Physics of Particles and Nuclei 41, 716.

Nikolskii, E., A. Korsheninnikov, H. Otsu, H. Suzuki, K. Yoneda, H. Baba, K. Yamada, Y. Kondo, N. Aoi, A. Denikin, M. Golovkov, A. Fomichev, et al., 2010, Phys.Rev. C 81, 064606.

Nilsson, T., G. Nyman, and K. Riisager, 2000, Hyperfine Interactions 129(1), 67.

Nolden, F., C. Dimopoulou, A. Dolinskii, and M. Steck, 2008, Nuclear Instruments and Methods in Physics Research Section B: Beam Interactions with Materials and Atoms 266(19-20), 4569

Nummela, S., F. Nowacki, P. Baumann, E. Caurier, J. Cederkäll, S. Courtin, P. Dessagne, A. Jokinen, A. Knipper, G. Le Scornet, L. G. Lyapin, C. Miehé, et al., 2001, Phys. Rev. C 64(5), 054313.

Nyman, G., R. E. Azuma, P. G. Hansen, B. Jonson, P. O. Larsson, S. Mattsson, A. Richter, K. Riisager, O. Tengblad, and K. Wilhelmsen, 1990, Nuclear Physics A 510(2), 189.

Oganessian, Y., 2007, Journal of Physics G: Nuclear and Particle Physics 34(4), R165.

Oganessian, Y. T., F. S. Abdullin, P. D. Bailey, D. E. Benker, M. E. Bennett, S. N. Dmitriev, J. G. Ezold, J. H. Hamilton, R. A. Henderson, M. G. Itkis, Y. V. Lobanov, A. N. Mezentsev, et al., 2010, Phys. Rev. Lett. 104(14), 142502.

Ohnishi, T., T. Kubo, K. Kusaka, A. Yoshida, K. Yoshida, M. Ohtake, N. Fukuda, H. Takeda, D. Kameda, K. Tanaka, N. Inabe, Y. Yanagisawa, et al., 2010, Journal of the Physical Society of Japan 79(7), 073201.

Okołowicz, J., M. Płoszajczak, and I. Rotter, 2003, Physics Reports 374(4-5), 271.

Ormand, W. E., 1996, Phys. Rev. C 53(1), 214.

Ormand, W. E., 1997, Phys. Rev. C 55, 2407.

Osterfeld, F., 1992, Rev. Mod. Phys. 64(2), 491.

Ozawa, A., G. Raimann, R. N. Boyd, F. R. Chloupek, M. Fujimaki, K. Kimura, H. Kitagawa, T. Kobayashi, J. J. Kolata, S. Kubono, I. Tanihata, Y. Watanabe, et al., 1995, Nuclear Physics A 592(2), 244.

Page, R., A. N. Andreyev, D. E. Appelbe, P. A. Butler, S. J. Freeman, P. T. Greenlees, R. D. Herzberg, D. G. Jenkins, G. D. Jones, P. Jones, D. T. Joss, R. Julin, et al., 2003, Nuclear Instruments and Methods in Physics Research Section B: Beam Interactions with Materials and Atoms 204, 634.

Page, R., L. Bianco, I. Darby, J. Uusitalo, D. Joss, T. Grahn, R.-D. Herzberg, J. Pakarinen, J. Thomson, S. Eeckhaudt, P. Greenlees, P. Jones, et al., 2007, Phys. Rev. C 75, 061302 .

Page, R., P. Woods, R. Cunningham, T. Davinson, N. Davis, S. Hofmann, A. James, K. Livingston, P. Sellin, and A. Shotter, 1992, Phys. Rev. Lett. 68, 1287.

Page, R., P. Woods, R. Cunningham, T. Davinson, N. Davis, A. James, K. Livingston, P. Sellin, and A. Shotter, 1994, Phys. Rev. Lett. 72, 1178. 
Page, R., P. Woods, R. Cunningham, T. Davinson, N. Davis, A. James, K. Livingston, P. Sellin, and A. Shotter, 1996, Phys. Rev. C 53, 660.

Page, R. D., 2011, Phys. Rev. C 83(1), 014305.

Pais, A., 1986, Inward bound: of matter and forces in the physical world (Oxford University Press).

Paul, E. S., P. J. Woods, T. Davinson, R. D. Page, P. J. Sellin, C. W. Beausang, R. M. Clark, R. A. Cunningham, S. A. Forbes, D. B. Fossan, A. Gizon, J. Gizon, et al., 1995, Phys. Rev. C 51(1), 78.

Pauli, W., 1930, in W. Pauli, collected scientific papers, edited by R. Kronig and V. Weisskopf (Interscience, New York, 1964), volume 2, p. 1313.

Pechenaya, O. L., C. J. Chiara, D. G. Sarantites, W. Reviol, R. J. Charity, M. P. Carpenter, R. V. F. Janssens, T. Lauritsen, C. J. Lister, D. Seweryniak, S. Zhu, L.-L. Andersson, et al., 2007, Phys. Rev. C 76, 011304(R).

Penttilä, H., P. Karvonen, T. Eronen, V. Elomaa, U. Hager, J. Hakala, A. Jokinen, A. Kankainen, I. Moore, K. Peräjärvi, S. Rahaman, S. Rinta-Antila, et al., 2010, The European Physical Journal A - Hadrons and Nuclei 44, 147.

Pereira, J., J. Benlliure, E. Casarejos, P. Armbruster, M. Bernas, A. Boudard, S. Czajkowski, T. Enqvist, R. Legrain, S. Leray, B. Mustapha, M. Pravikoff, et al., 2007, Phys. Rev. C 75(1), 014602.

Pereira, J., P. Hosmer, G. Lorusso, P. Santi, A. Couture, J. Daly, M. D. Santo, T. Elliot, J. Görres, C. Herlitzius, K.L. Kratz, L. Lamm, et al., 2010, Nuclear Instruments and Methods in Physics Research Section A: Accelerators, Spectrometers, Detectors and Associated Equipment 618(1-3), 275.

Pervin, M., S. C. Pieper, and R. B. Wiringa, 2007, Phys. Rev. C 76(6), 064319.

Pfeiffer, B., K. L. Kratz, F. K. Thielemann, and W. B. Walters, 2001, Nuclear Physics A 693(1-2), 282.

Pfennig, G., C. Normand, J. Magill, and T. Fanghänel (eds.), 2008, Karlsruher Nuklidkarte. Commemoration of the 50th Anniversary (Luxembourg: Office for Official Publications of the European Communities).

Pfützner, M., E. Badura, C. Bingham, B. Blank, M. Chartier, H. Geissel, J. Giovinazzo, L. Grigorenko, R. Grzywacz, M. Hellström, Z. Janas, J. Kurcewicz, et al., 2002, Eur. Phys. J. A 14, 279.

Pühlhofer, F., 1977, Nuclear Physics A 280(1), 267.

Pietri, S., P. Regan, Z. Podolyák, D. Rudolph, S. Steer, A. Garnsworthy, E. Werner-Malento, R. Hoischen, M. Górska, J. Gerl, H. Wollersheim, I. Kojouharov, et al., 2007, Nuclear Instruments and Methods in Physics Research Section B: Beam Interactions with Materials and Atoms 261(1-2), 1079.

Poenaru, D. N., Y. Nagame, R. A. Gherghescu, and W. Greiner, 2002, Phys. Rev. C 65(5), 054308.

Poli, G., C. Davids, P. Woods, D. Seweryniak, J. Batchelder, L. Brown, C. Bingham, M. Carpenter, L. Conticchio, T. Davinson, J. DeBoer, S. Hamada, et al., 1999, Phys. Rev. C 59, R2979.

Poli, G., C. Davids, P. Woods, D. Seweryniak, M. Carpenter, J. Ciżewski, T. Davinson, A. Heinz, R. Janssens, C. Lister, J. Ressler, A. Sonzogni, et al., 2001, Phys. Rev. C 63(4), 044304.

Pomorski, M., K. Miernik, W. Dominik, Z. Janas, M. Pfützner, C. R. Bingham, H. Czyrkowski, M. Ćwiok, I. G. Darby, R. Dąbrowski, T. Ginter, R. Grzywacz, et al., 2011a, Phys. Rev. C 83(1), 014306.

Pomorski, M., M. Pfützner, W. Dominik, R. Grzywacz, T. Baumann, J. S. Berryman, H. Czyrkowski, R. Dąbrowski, T. Ginter, J. Johnson, G. Kamiński, A. Kuźniak, et al., 2011b, Phys. Rev. C 83(6), 061303.

Prezado, Y., U. C. Bergmann, M. J. G. Borge, J. Cederkäll, C. A. Diget, L. M. Fraile, H. O. U. Fynbo, H. Jeppesen, B. Jonson, M. Meister, T. Nilsson, G. Nyman, et al., 2003, Physics Letters B 576(1-2), 55.

Prezado, Y., M. J. G. Borge, C. A. Diget, L. M. Fraile, B. R. Fulton, H. O. U. Fynbo, H. B. Jeppesen, B. Jonson, M. Meister, T. Nilsson, G. Nyman, K. Riisager, et al., 2005, Physics Letters B 618(1-4), 43.

Qi, C., F. R. Xu, R. J. Liotta, and R. Wyss, 2009a, Phys. Rev. Lett. 103(7), 072501.

Qi, C., F. R. Xu, R. J. Liotta, R. Wyss, M. Y. Zhang, C. Asawatangtrakuldee, and D. Hu, 2009b, Phys. Rev. C $\mathbf{8 0}(4), 044326$.

Raabe, R., A. Andreyev, M. J. G. Borge, L. Buchmann, P. Capel, H. O. U. Fynbo, M. Huyse, R. Kanungo, T. Kirchner, C. Mattoon, A. C. Morton, I. Mukha, et al., 2008, Phys. Rev. Lett. 101(21), 212501.

Raabe, R., J. Büscher, J. Ponsaers, F. Aksouh, M. Huyse, O. Ivanov, S. R. Lesher, I. Mukha, D. Pauwels, M. Sawicka, D. Smirnov, I. Stefanescu, et al., 2009, Phys. Rev. C 80(5), 054307.

Raciti, G., G. Cardella, M. De Napoli, E. Rapisarda, F. Amorini, and C. Sfienti, 2008, Phys. Rev. Lett. 100(19), 192503.

Radivojevic, Z., P. Baumann, E. Caurier, J. Cederkäll, S. Courtin, P. Dessagne, A. Jokinen, A. Knipper, G. L. Scornet, V. Lyapin, C. Miehé, F. Nowacki, et al., 2002, Nuclear Instruments and Methods in Physics Research Section A: Accelerators, Spectrometers, Detectors and Associated Equipment 481(1-3), 464.

Raimann, G., A. Ozawa, R. N. Boyd, F. R. Chloupek, M. Fujimaki, K. Kimura, T. Kobayashi, J. J. Kolata, S. Kubono, I. Tanihata, Y. Watanabe, and K. Yoshida, 1996, Phys. Rev. C 53(1), 453.

Rasmussen, J., 1966, Alpha-, Beta-, and Gamma-Ray Spectroscopy (North-Holland), p. 701.

Reed, A. T., O. Tarasov, R. D. Page, D. Guillemaud-Mueller, Y. E. Penionzhkevich, R. G. Allatt, J. C. Angélique, R. Anne, C. Borcea, V. Burjan, W. N. Catford, Z. Dlouhý, et al., 1999, Phys. Rev. C 60(2), 024311.

Reisdorf, W., 1981, Zeitschrift für Physik A Hadrons and Nuclei 300, 227.

Reisdorf, W., and M. Schädel, 1992, Zeitschrift für Physik A Hadrons and Nuclei 343, 47.

Reiter, P., T. L. Khoo, C. J. Lister, D. Seweryniak, I. Ahmad, M. Alcorta, M. P. Carpenter, J. A. Cizewski, C. N. Davids, G. Gervais, J. P. Greene, W. F. Henning, et al., 1999, Phys. Rev. Lett. 82(3), 509.

Ricciardi, M. V., P. Armbruster, J. Benlliure, M. Bernas, A. Boudard, S. Czajkowski, T. Enqvist, A. Kelić, S. Leray, R. Legrain, B. Mustapha, J. Pereira, et al., 2006, Phys. Rev. C 73(1), 014607.

Robinson, A., C. Davids, G. Mukherjee, D. Seweryniak, S. Sinha, P. Wilt, and P. Woods, 2003, Phys. Rev. C 68, 054301.

Robinson, A., P. Woods, D. Seweryniak, C. Davids, M. Carpenter, A. Hecht, D. Peterson, S. Sinha, W. Walters, and S. Zhu, 2005, Phys. Rev. Lett. 95, 032502.

Robson, D., 1975, in Nuclear Spectroscopy and Reactions, edited by J. Cerny (Academic Press), volume D, pp. 179- 
248.

Rochman, D., I. Tsekhanovich, F. Gönnenwein, V. Sokolov, F. Storrer, G. Simpson, and O. Serot, 2004, Nuclear Physics A 735(1-2), 3.

Rodin, A. M., S. V. Stepantsov, D. D. Bogdanov, M. S. Golovkov, A. S. Fomichev, S. I. Sidorchuk, R. S. Slepnev, R. Wolski, G. M. Ter-Akopian, Y. T. Oganessian, A. A. Yukhimchuk, V. V. Perevozchikov, et al., 2003, Nuclear Instruments and Methods in Physics Research Section B: Beam Interactions with Materials and Atoms 204, 114.

Roeckl, E., 1995, Radiochimica Acta 70/71, 107.

Roeckl, E., 1996, Nuclear Decay Modes. (Institute of Physics Publishing), chapter Alpha Decay, pp. 237-274.

Rogers, A. M., M. A. Famiano, W. G. Lynch, M. S. Wallace, F. Amorini, D. Bazin, R. J. Charity, F. Delaunay, R. T. de Souza, J. Elson, A. Gade, D. Galaviz, et al., 2011, Phys. Rev. Lett. 106(25), 252503.

Rose, H., and G. Jones, 1984, Nature 307, 245.

Rotureau, J., J. Okołowicz, and M. Płoszajczak, 2005, Phys. Rev. Lett. 95, 042503, 4 pages.

Rotureau, J., J. Okołowicz, and M. Płoszajczak, 2006, Nuclear Physics A 767, 13 , ISSN 0375-9474.

Rubio, B., W. Gelletly, E. Nácher, A. Algora, J. L. Taín, A. Pérez, and L. Caballero, 2005, Journal of Physics G: Nuclear and Particle Physics 31(10), S1477.

Rudolph, D., 2002, The European Physical Journal A Hadrons and Nuclei 15, 161.

Rudolph, D., R. Hoischen, M. Hellström, S. Pietri, Z. Podolyák, P. H. Regan, A. B. Garnsworthy, S. J. Steer, F. Becker, P. Bednarczyk, L. Cáceres, P. Doornenbal, et al., 2008, Phys. Rev. C 78(2), 021301.

Rutherford, E., 1899, Phil. Mag. 47, 109.

Rutherford, E., 1911, Phil. Mag. 21, 669.

Rutherford, E., and E. da C. Andrade, 1914, Phil. Mag. 27, 854.

Rutherford, E., and H. Geiger, 1908, Proc. Roy. Soc. A81, 162.

Rykaczewski, K., 2002, Eur. Phys. J. A 15, 81.

Rykaczewski, K., J. Batchelder, C. Bingham, T. Davinson, T. Ginter, C. Gross, R. Grzywacz, M. Karny, B. MacDonald, J. Mas, J. McConnell, A. Piechaczek, et al., 1999, Phys. Rev. C 60, 011301.

Rykaczewski, K., J. Batchelder, C. Bingham, T. Ginter, C. Gross, R. Grzywacz, J. Hamilton, D. Hartley, Z. Janas, M. Karny, W. Kulp, M. Lipoglavsek, et al., 2001a, Acta Phys. Pol. B 32, 971.

Rykaczewski, K., R. Grzywacz, M. Karny, J. McConnell, M. Momayezi, J. Wahl, Z. Janas, J. Batchelder, C. B. D. Hartley, M. Tantawy, C. Gross, T. Ginter, et al., 2001b, Nucl. Phys. A 682, 270c.

Sagawa, H., I. Hamamoto, and M. Ishihara, 1993, Physics Letters B 303(3-4), 215.

Sakurai, H., 2008, Nuclear Physics A 805(1-4), 526c.

Schardt, D., and K. Riisager, 1993, Zeitschrift für Physik A Hadrons and Nuclei 345, 265.

Schatz, H., A. Aprahamian, J. Görres, M. Wiescher, T. Rauscher, J. F. Rembges, F. K. Thielemann, B. Pfeiffer, P. Möller, K. L. Kratz, H. Herndl, B. A. Brown, et al., 1998, Physics Reports 294(4), 167.

Schmidt, K. H., J. Benlliure, T. Enqvist, A. R. Junghans, F. Rejmund, and M. V. Ricciardi, 2002, Nuclear Physics A 701(1-4), 115.

Schmidt, K. H., T. Brohm, H. G. Clerc, M. Dornik, M. Fauerbach, H. Geissel, A. Grewe, E. Hanelt, A. Junghans,
A. Magel, W. Morawek, G. Münzenberg, et al., 1993, Physics Letters B 300(4), 313.

Schmidt, K. H., and W. Morawek, 1991, Reports on Progress in Physics 54(7), 949.

Schneider, R., J. Friese, J. Reinhold, K. Zeitelhack, T. Faestermann, R. Gernhäuser, H. Gilg, F. Heine, J. Homolka, P. Kienle, H. J. Körner, H. Geissel, et al., 1994, Zeitschrift für Physik A Hadrons and Nuclei 348, 241.

Scholey, C., M. Sandzelius, S. Eeckhaudt, T. Grahn, P. Greenlees, P. Jones, R. Julin, S. Juutinen, M. Leino, A.-P. Leppänen, P. Nieminen, M. Nyman, et al., 2005, J. Phys. G 31, S1719.

Sellin, P., P. Woods, T. Davinson, N. Davis, K. Livingston, R. Page, A. Shotter, S. Hofmann, and A. James, 1993, Phys. Rev. C 47, 1933.

Sellin, P. J., P. J. Woods, D. Branford, T. Davinson, N. J. Davis, D. G. Ireland, K. Livingston, R. D. Page, A. C. Shotter, S. Hofmann, R. A. Hunt, A. N. James, et al., 1992, Nuclear Instruments and Methods in Physics Research Section A: Accelerators, Spectrometers, Detectors and Associated Equipment 311(1-2), 217.

Severijns, N., M. Beck, and O. Naviliat-Cuncic, 2006, Rev. Mod. Phys. 78(3), 991.

Seweryniak, D., D. Ackermann, H. Amro, L. T. Brown, M. P. Carpenter, L. Conticchio, C. N. Davids, S. M. Fischer, G. Hackman, S. Hamada, D. J. Henderson, R. V. F. Janssens, et al., 1998, Phys. Rev. C 58(5), 2710.

Seweryniak, D., B. Blank, M. Carpenter, C. Davids, T. Davinson, S. Freeman, N. Hammond, N. Hoteling, R. Janssens, T. Khoo, Z. Liu, G. Mukherjee, et al., 2007a, Phys. Rev. Lett. 99, 082502.

Seweryniak, D., M. P. Carpenter, S. Gros, A. A. Hecht, N. Hoteling, R. V. F. Janssens, T. L. Khoo, T. Lauritsen, C. J. Lister, G. Lotay, D. Peterson, A. P. Robinson, et al., 2007b, Phys. Rev. Lett. 99(2), 022504.

Seweryniak, D., C. Davids, A. Robinson, P. Woods, B. Blank, M. Carpenter, T. Davinson, S. Freeman, N. Hammond, N. Hoteling, R. Janssens, T. Khoo, et al., 2005a, Eur. Phys. J. A 25, 159 .

Seweryniak, D., C. N. Davids, W. B. Walters, P. J. Woods, I. Ahmad, H. Amro, D. J. Blumenthal, L. T. Brown, M. P. Carpenter, T. Davinson, S. M. Fischer, D. J. Henderson, et al., 1997, Phys. Rev. C 55(5), R2137.

Seweryniak, D., K. Starosta, C. N. Davids, S. Gros, A. A. Hecht, N. Hoteling, T. L. Khoo, K. Lagergren, G. Lotay, D. Peterson, A. Robinson, C. Vaman, et al., 2006, Phys. Rev. C 73(6), 061301.

Seweryniak, D., J. Uusitalo, P. Bhattacharyya, M. P. Carpenter, J. A. Cizewski, K. Y. Ding, C. N. Davids, N. Fotiades, R. V. F. Janssens, T. Lauritsen, C. J. Lister, A. O. Macchiavelli, et al., 2005b, Phys. Rev. C 71(5), 054319.

Seweryniak, D., P. Woods, J. Ressler, C. Davids, A. Heinz, J. Uusitalo, W. Walters, J. Caggiano, M. Carpenter, J. Cizewski, T. Davinson, K. Ding, et al., 2001, Phys. Rev. Lett. 86, 1458.

Shaughnessy, D. A., K. E. Gregorich, J. L. Adams, M. R. Lane, C. A. Laue, D. M. Lee, C. A. McGrath, V. Ninov, J. B. Patin, D. A. Strellis, E. R. Sylwester, P. A. Wilk, et al., 2002, Phys. Rev. C 65(2), 024612.

Shotter, A., 2003, Nuclear Instruments and Methods in Physics Research Section B: Beam Interactions with Materials and Atoms 204, 17.

Skeppstedt, O., H. A. Roth, L. Lindström, R. Wadsworth, I. Hibbert, N. Kelsall, D. Jenkins, H. Grawe, M. Górska, 
M. Moszynski, Z. Sujkowski, D. Wolski, et al., 1999, Nuclear Instruments and Methods in Physics Research Section A: Accelerators, Spectrometers, Detectors and Associated Equipment 421(3), 531.

Sonzogni, A., 2002, Nuclear Data Sheets 95, 1.

Sonzogni, A., C. Davids, P. Woods, D. Seweryniak, M. Carpenter, J. Ressler, J. Schwartz, J. Uusitalo, and W. Walters, 1999, Phys. Rev. Lett. 83, 1116.

Soramel, F., A. Guglielmetti, L. Stroe, L. Muller, R. Bonetti, G. Poli, F. Malerba, E. Bianchi, A. Andrighetto, J. Guo, Z. Li, E. Maglione, et al., 2001, Phys. Rev. C 63, 031304.

Sorlin, O., C. Donzaud, F. Azaiez, C. Bourgeois, L. Gaudefroy, F. Ibrahim, D. Guillemaud-Mueller, F. Pougheon, M. Lewitowicz, F. de Oliveira Santos, M. G. Saint-Laurent, M. Stanoiu, et al., 2003, Nuclear Physics A 719, C193.

Sorlin, O., and M. G. Porquet, 2008, Prog.Part.Nucl.Phys. 61, 602 .

Stanoiu, M., K. Sümmerer, I. Mukha, A. Chatillon, E. C. Gil, M. Heil, J. Hoffman, O. Kiselev, N. Kurz, and W. Ott, 2008, Nuclear Instruments and Methods in Physics Research Section B: Beam Interactions with Materials and Atoms 266(19-20), 4625.

Starosta, K., C. Vaman, D. Miller, P. Voss, D. Bazin, T. Glasmacher, H. Crawford, P. Mantica, H. Tan, W. Hennig, M. Walby, A. Fallu-Labruyere, et al., 2009, Nuclear Instruments and Methods in Physics Research Section A: Accelerators, Spectrometers, Detectors and Associated Equipment 610(3), 700 .

Stracener, D. W., 2003, Nuclear Instruments and Methods in Physics Research Section B: Beam Interactions with Materials and Atoms 204, 42.

Sumithrarachchi, C. S., D. J. Morrissey, A. D. Davies, D. A. Davies, M. Facina, E. Kwan, P. F. Mantica, M. Portillo, Y. Shimbara, J. Stoker, and R. R. Weerasiri, 2010, Phys. Rev. C 81(1), 014302.

Sümmerer, K., and B. Blank, 2000, Phys. Rev. C 61(3), 034607.

Sun, Z., W. L. Zhan, Z. Y. Guo, G. Xiao, and J. X. Li, 2003, Nuclear Instruments and Methods in Physics Research Section A: Accelerators, Spectrometers, Detectors and Associated Equipment 503(3), 496.

Suzuki, D., H. Iwasaki, D. Beaumel, L. Nalpas, E. Pollacco, M. Assie, H. Baba, Y. Blumenfeld, N. D. Sereville, A. Drouart, S. Franchoo, A. Gillibert, et al., 2009, Phys. Rev. Lett. 103, 152503.

Symons, T. J. M., Y. P. Viyogi, G. D. Westfall, P. Doll, D. E. Greiner, H. Faraggi, P. J. Lindstrom, D. K. Scott, H. J. Crawford, and C. McParland, 1979, Phys. Rev. Lett. 42(1), 40.

Taieb, J., K. H. Schmidt, L. Tassan-Got, P. Armbruster, J. Benlliure, M. Bernas, A. Boudard, E. Casarejos, S. Czajkowski, T. Enqvist, R. Legrain, S. Leray, et al., 2003, Nuclear Physics A 724(3-4), 413.

Tanihata, I., 2008, Nuclear Instruments and Methods in Physics Research Section B: Beam Interactions with Materials and Atoms 266(19-20), 4067.

Tantawy, M., C. Bingham, K. Rykaczewski, J. Batchelder, W. Królas, M. Danchev, D. Fong, T. Ginter, C. Gross, R. Grzywacz, K. Hagino, J. Hamilton, et al., 2006, Phys. Rev. C 73, 024316.

Tarasov, O. B., D. J. Morrissey, A. M. Amthor, T. Baumann, D. Bazin, A. Gade, T. N. Ginter, M. Hausmann, N. Inabe, T. Kubo, A. Nettleton, J. Pereira, et al., 2009, Phys. Rev. Lett. 102(14), 142501.
Tengblad, O., U. C. Bergmann, L. M. Fraile, H. O. U. Fynbo, and S. Walsh, 2004, Nuclear Instruments and Methods in Physics Research Section A: Accelerators, Spectrometers, Detectors and Associated Equipment 525(3), 458.

Thaysen, J., L. Axelsson, J. Äystö, M. J. G. Borge, L. M. Fraile, H. O. U. Fynbo, A. Honkanen, P. Hornshøj, Y. Jading, A. Jokinen, B. Jonson, I. Martel, et al., 1999, Physics Letters B 467(3-4), 194.

Thibault, C., R. Klapisch, C. Rigaud, A. M. Poskanzer, R. Prieels, L. Lessard, and W. Reisdorf, 1975, Phys. Rev. C 12(2), 644 .

Thoennessen, M., 2004, Reports on Progress in Physics 67(7), 1187.

Thoennessen, M., 2010, Nuclear Physics A 834(1-4), 688c.

Tilley, D., H. Weller, and G. Hale, 1992, Nucl. Phys. A541, 1.

Toivanen, J., B. G. Carlsson, J. Dobaczewski, K. Mizuyama, R. R. Rodríguez-Guzmán, P. Toivanen, and P. Veselý, 2010, Phys. Rev. C 81(3), 034312.

Toth, K., D. Sousa, P. Wilmarth, J. Nitschke, and K. Vierinen, 1993, Phys. Rev. C 47, 1804.

Towner, I. S., and J. C. Hardy, 2010, Reports on Progress in Physics 73(4), 046301.

Trinder, W., E. G. Adelberger, B. A. Brown, Z. Janas, H. Keller, K. Krumbholz, V. Kunze, P. Magnus, F. Meissner, A. Piechaczek, M. Pfützner, E. Roeckl, et al., 1997, Nuclear Physics A 620(2), 191.

Tripathi, V., S. L. Tabor, C. R. Hoffman, M. Wiedeking, A. Volya, P. F. Mantica, A. D. Davies, S. N. Liddick, W. F. Mueller, A. Stolz, B. E. Tomlin, T. Otsuka, et al., 2006, Phys. Rev. C 73(5), 054303.

Tursunov, E. M., D. Baye, and P. Descouvemont, 2006a, Phys. Rev. C 73(1), 014303.

Tursunov, E. M., D. Baye, and P. Descouvemont, 2006b, Phys. Rev. C 74(6), 069904.

Uusitalo, J., C. Davids, P. Woods, D. Seweryniak, A. Sonzogni, J. Batchelder, C. Bingham, T. Davinson, J. de Boer, D. Henderson, H. Maier, J. Ressler, et al., 1999, Phys. Rev. C 59(6), R2975.

Varga, K., R. G. Lovas, and R. J. Liotta, 1992, Phys. Rev. Lett. 69(1), 37.

Vasilevsky, V., A. Nesterov, F. Arickx, and J. Broeckhove, 2001, Phys. Rev. C 63, 034607.

Villard, P., 1900, Comptes Rendus 130, 1010,1178.

Villari, A. C. C., 2003, Nuclear Instruments and Methods in Physics Research Section B: Beam Interactions with Materials and Atoms 204, 31.

Volya, A., and V. Zelevinsky, 2006, Phys. Rev. C 74, 064314.

Voulot, D., F. Wenander, E. Piselli, R. Scrivens, M. Lindroos, H. Jeppesen, L. Fraile, S. Sturm, and P. Delahaye, 2008, Nuclear Instruments and Methods in Physics Research Section B: Beam Interactions with Materials and Atoms 266(19-20), 4103.

Wahl, A. C., 1988, Atomic Data and Nuclear Data Tables $39(1), 1$.

Wallace, M., M. Famiano, M.-J. van Goethem, A. Rogers, W. Lynch, J. Clifford, F. Delaunay, J. Lee, S. Labostov, M. Mocko, L. Morris, A. Moroni, et al., 2007, Nuclear Instruments and Methods in Physics Research Section A: Accelerators, Spectrometers, Detectors and Associated Equipment 583(2-3), 302.

Warburton, W., and P. Grudberg, 2006, Nuclear Instruments and Methods in Physics Research Section A: Accelerators, Spectrometers, Detectors and Associated Equipment 
$\mathbf{5 6 8}(1), 350$

Webber, W. R., J. C. Kish, and D. A. Schrier, 1990, Phys. Rev. C 41(2), 520.

Weidenmüller, H. A., and G. E. Mitchell, 2009, Rev. Mod. Phys. 81(2), 539 .

Weissman, L., U. Bergmann, J. Cederkall, L. M. Fraile, S. Franchoo, H. Fynbo, H. Gausemel, H. Jeppesen, U. Köster, K.-L. Kratz, T. Nilsson, B. Pfeiffer, et al., 2003, Phys. Rev. C 67(5), 054314.

Wenander, F., 2008, Nuclear Instruments and Methods in Physics Research Section B: Beam Interactions with Materials and Atoms 266(19-20), 4346.

Westfall, G. D., T. J. M. Symons, D. E. Greiner, H. H. Heckman, P. J. Lindstrom, J. Mahoney, A. C. Shotter, D. K. Scott, H. J. Crawford, C. McParland, T. C. Awes, C. K. Gelbke, et al., 1979, Phys. Rev. Lett. 43(25), 1859.

Whaling, W., 1966, Phys. Rev. 150, 836.

Wick, G.-C., 1934, Rend. Acc. Lincei 19, 319.

Wilkinson, D. H., 1995, Nuclear Instruments and Methods in Physics Research Section A: Accelerators, Spectrometers, Detectors and Associated Equipment 365(2-3), 497.

Winger, J. A., S. V. Ilyushkin, K. P. Rykaczewski, C. J. Gross, J. C. Batchelder, C. Goodin, R. Grzywacz, J. H. Hamilton, A. Korgul, W. Królas, S. N. Liddick, C. Mazzocchi, et al., 2009, Phys. Rev. Lett. 102(14), 142502.

Winkler, M., H. Geissel, H. Weick, B. Achenbach, K.-H. Behr, D. Boutin, A. Brünle, M. Gleim, W. Hüller, C. Karagiannis, A. Kelic, B. Kindler, et al., 2008, Nuclear Instruments and Methods in Physics Research Section B: Beam Interactions with Materials and Atoms 266(19-20), 4183.

Woods, P., T. Davinson, N. Davis, S. Hofmann, A. James, K. Livingston, R. Page, P. Selin, and A. Shotter, 1993, Nucl. Phys. A 553, 485c.

Woods, P., and C. Davis, 1997, Annu. Rev. Nucl. Part. Sci. 47, 541.

Woods, P., P. Munro, D. Seweryniak, C. Davids, T. D. A. Heinz, H. Mahud, F. Sarazin, J. Shergur, W. Walters, and
A. Woehr, 2004, Phys. Rev. C 69, 051302(R).

Woodward, C., R. Tribble, and D. Tanner, 1983, Phys. Rev. C 27, 27.

Wormer, L. V., J. Görres, C. Iliadis, M. Wiescher, and F.-K. Thielemann, 1994, The Astrophysical Journal 432, 326.

Wrede, C., J. A. Caggiano, J. A. Clark, C. M. Deibel, A. Parikh, and P. D. Parker, 2009, Phys. Rev. C 79(4), 045808 .

Wu, C. S., E. Ambler, R. W. Hayward, D. D. Hoppes, and R. P. Hudson, 1957, Phys. Rev. 105(4), 1413.

Yoneda, K., N. Aoi, H. Iwasaki, H. Sakurai, H. Ogawa, T. Nakamura, W.-D. Schmidt-Ott, M. Schäfer, M. Notani, N. Fukuda, E. Ideguchi, T. Kishida, et al., 2003, Phys. Rev. C 67(1), 014316.

Yordanov, D. T., M. Kowalska, K. Blaum, M. De Rydt, K. T. Flanagan, P. Lievens, R. Neugart, G. Neyens, and H. H. Stroke, 2007, Phys. Rev. Lett. 99(21), 212501.

Äystö, J., 2001, Nuclear Physics A 693(1-2), 477.

Yu, C.-H., J. Batchelder, C. Bingham, R. Grzywacz, K. Rykaczewski, K. Toth, Y. Akovali, C. Baktash, A. Galindo-Uribarri, T. Ginter, C. Gross, M. Karny, et al., 1998, Phys. Rev. C 58, R3042.

Zagrebaev, V., and W. Greiner, 2008, Phys. Rev. C 78(3), 034610.

Zeldovich, Y. B., 1960, JETP 38, 1123.

Zelevinsky, V., 1996, Annual Review of Nuclear and Particle Science 46, 237.

Zerguerras, T., B. Blank, Y. Blumenfeld, T. Suomijärvi, D. Beaumel, B. Brown, M. Chartier, M. Fallot, J. Giovinazzo, C. Jouanne, V. Lapoux, I. Lhenry-Yvon, et al., 2004, Eur. Phys. J. A 20(3), 389.

Zhang, X., Z. Ren, Q. Zhi, and Q. Zheng, 2007, Journal of Physics G: Nuclear and Particle Physics 34(12), 2611.

Zhukov, M. V., B. V. Danilin, D. V. Fedorov, J. M. Bang, I. J. Thompson, and J. S. Vaagen, 1993, Physics Reports 231(4), 151 . 